\title{
User's Manual for the NEFTRAN II Computer Code
}

Manuscript Completed: January 1991

Date Published: February 1991

Prepared by

N. E. Olague, D. E. Longsine' ${ }^{1}$ J. E. Campbell, C. D. Leigh

Sandia National Laboratories

Albuquerque, NM 87185

Prepared for

Division of Engineering

Office of Nuclear Regulatory Research

U.S. Nuclear Regulatory Commission

Washington, DC 20555

NRC FIN A1266

IIntera, Inc., Austin, TX 


\section{DISCLAIMER}

This report was prepared as an account of work sponsored by an agency of the United States Government. Neither the United States Government nor any agency thereof, nor any of their employees, make any warranty, express or implied, or assumes any legal liability or responsibility for the accuracy, completeness, or usefulness of any information, apparatus, product, or process disclosed, or represents that its use would not infringe privately owned rights. Reference herein to any specific commercial product, process, or service by trade name, trademark, manufacturer, or otherwise does not necessarily constitute or imply its endorsement, recommendation, or favoring by the United States Government or any agency thereof. The views and opinions of authors expressed herein do not necessarily state or reflect those of the United States Government or any agency thereof. 


\section{DISCLAIMER}

Portions of this document may be illegible in electronic image products. Images are produced from the best available original document. 
This document describes the NEFTRAN II (NEtwork Flow and TRANsport in Time-Dependent Velocity Fields) computer code and is intended to provide the reader with sufficient information to use the code. NEFTRAN II was developed as part of a performance assessment methodology for storage of high-level nuclear waste in unsaturated, welded tuff. NEFTRAN II is a successor to the NEFTRAN and NWFT/DVM computer codes and contains several new capabilities. These capabilities include: 1) the ability to input pore velocities directly to the transport model and bypass the network fluid flow model, 2) the ability to transport radionuclides in timedependent velocity fields, 3) the ability to account for the effect of time-dependent saturation changes on the retardation factor, and 4) the ability to account for time-dependent flow rates through the source regime. In addition to these changes, the input to NEFTRAN II has been modified to be more convenient for the user. This document is divided into four main sections consisting of 1) a description of all the models contained in the code, 2) a description of the program and subprograms in the code, 3) a data input guide and 4) verification and sample problems. Although NEFTRAN II is the fourth generation code, this document is a complete description of the code and reference to past user's manuals should not be necessary. 


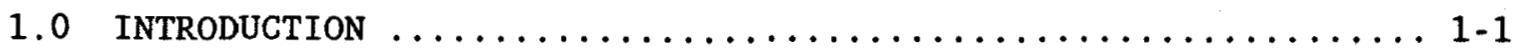

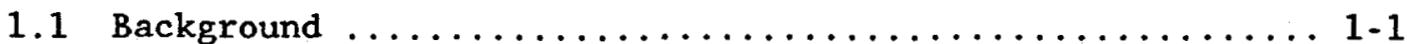

1.2 Purpose ............................... 1-1

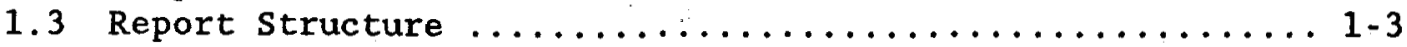

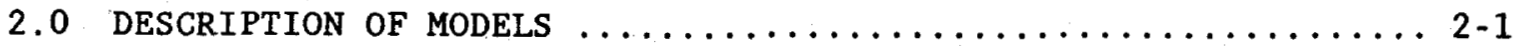

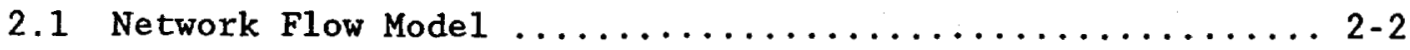

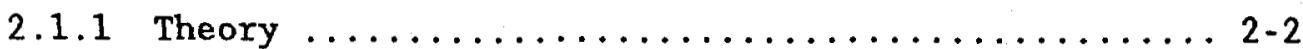

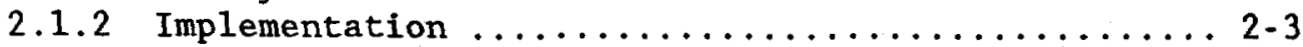

2.1.3 Fluid Density and Viscosity Submodels $\ldots \ldots \ldots \ldots 2-5$

2.2 Analytical Transport Model $\ldots \ldots \ldots \ldots \ldots \ldots \ldots \ldots \ldots \ldots$ 2-7

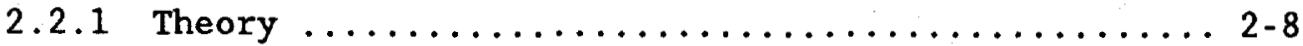

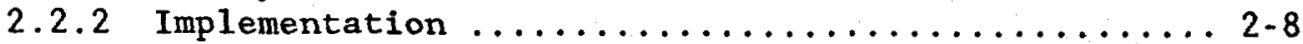

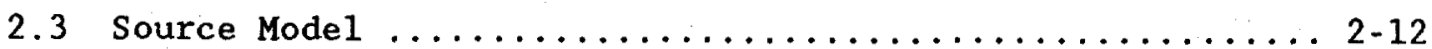

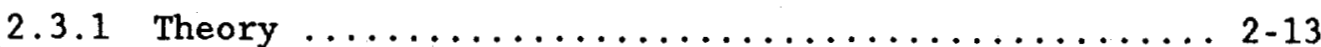

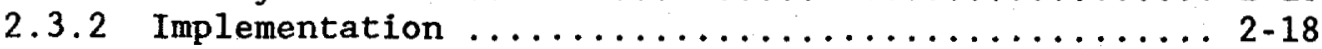

2.4 Distributed Velocity Method (DVM) Transport Mode1 ...... 2-21

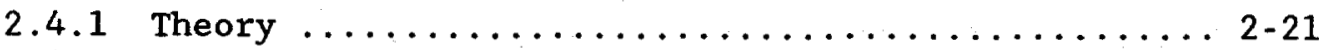

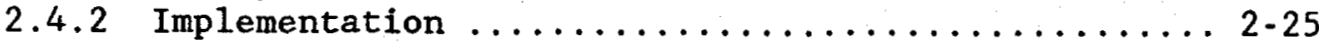

2.4.3 Time Step Determination ................. 2-33

2.4 .4 Spatial-Step Determination .................... 2-35

2.4.5 Courant Numbers and Isotope Travel Times ........ 2-36

2.4 .6 Species Velocity Model ................... 2-47

2.4 .7 Discharge Model ...................... 2-49

2.4.8 Distribution of Source Into Grid Blocks ........ 2-49

2.4.9 Leg-to-Leg Transfer Mode1 ............... 2-50

2.4.10 Matrix Diffusion Mode1................... 2-54

2.4.11 DVM Transport in Time-Dependent Velocity

Fields ........................... 2-59

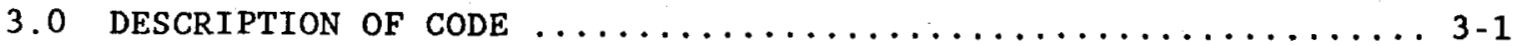

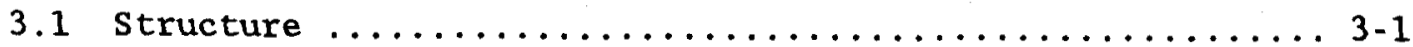

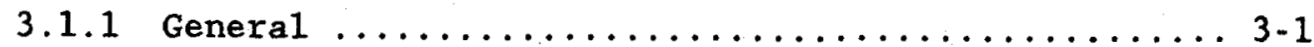

3.1 .2 Source Module ......................

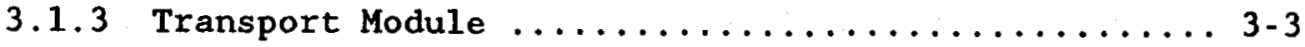


3.2 subroutines $\ldots \ldots \ldots \ldots \ldots \ldots \ldots \ldots \ldots \ldots \ldots \ldots \ldots \ldots \ldots$

3.2.1 NEFMAIN - main program $\ldots \ldots \ldots \ldots \ldots \ldots \ldots \ldots \ldots$ 3-6

3.2 .2 ADJB(VA, LG, IP, TS, ILAST, KNT) $\ldots \ldots \ldots \ldots \ldots \ldots$

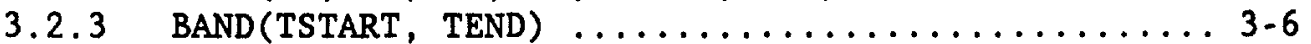

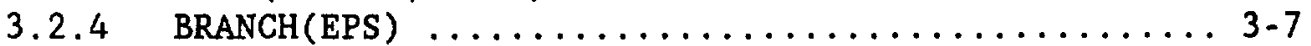

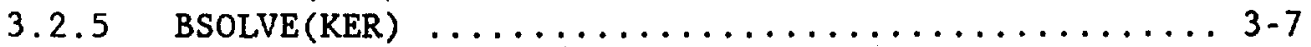

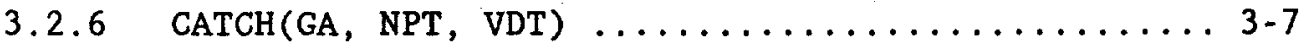

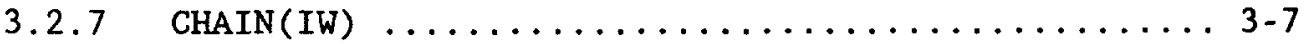

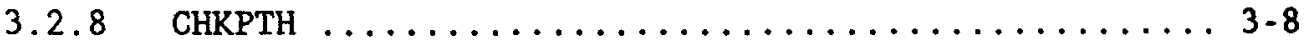

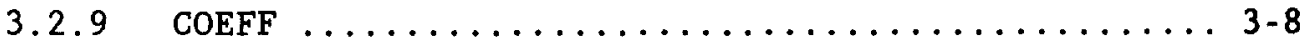

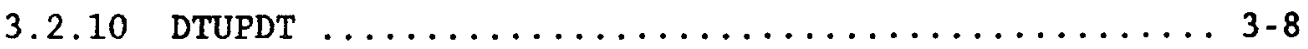

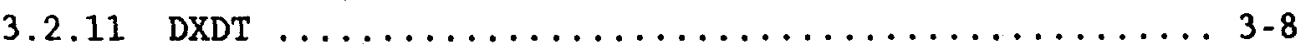

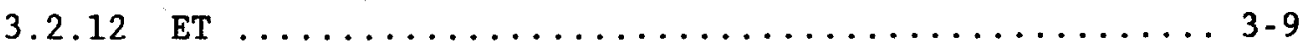

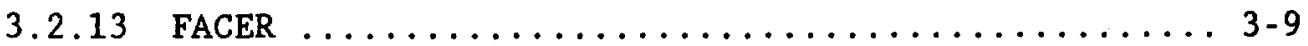

3.2.14 FLOWIN(IPASS, ROOT, KROOT) ............. 3-9

3.2.15 GETRV(JTRIAL) ..................... 3-10

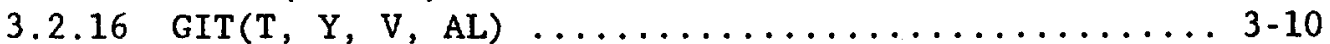

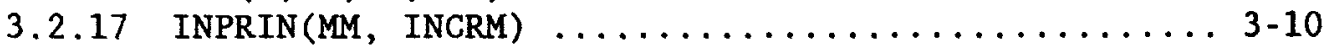

3.2.18 INTG(S1, B1, S2, B2, T1, T2, D, F, N, DT, ANS) $\ldots \ldots \ldots \ldots .3-10$

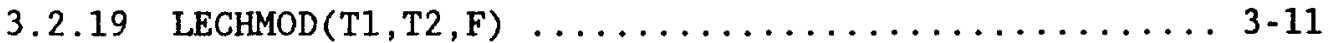

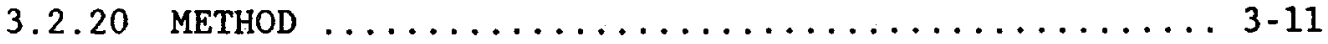

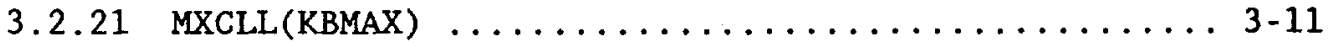

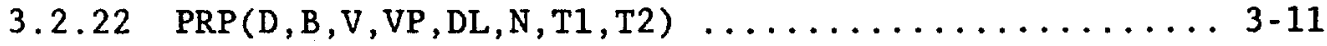

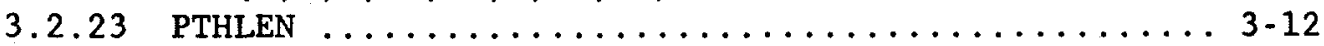

3.2.24 RATIO(DJ, DI, TIME, RAT) $\ldots \ldots \ldots \ldots \ldots \ldots \ldots \ldots \ldots \ldots .12$

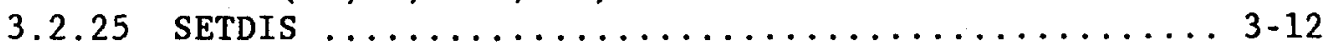

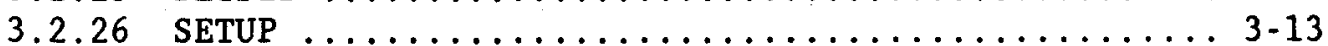

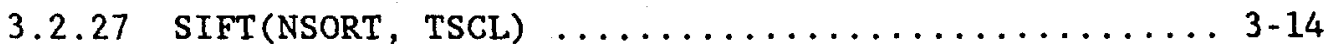

3.2 .28 SOURCE(IPASS, JTRIAL) $\ldots \ldots \ldots \ldots \ldots \ldots \ldots \ldots \ldots \ldots \ldots \ldots \ldots .14$

3.2.29 SRCIN(T, SR1, SR2, TS1, TS2, N1, N2, NTOTX, S24, IEOF) . . 3-15

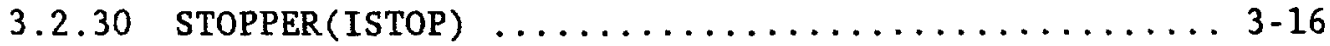

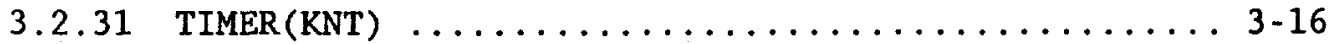

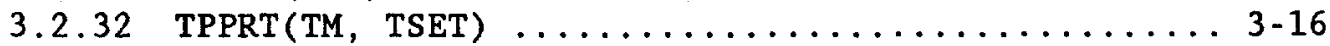

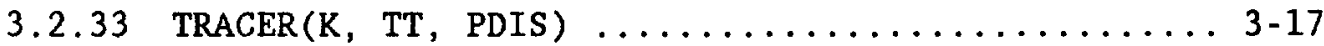

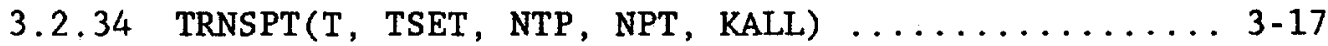

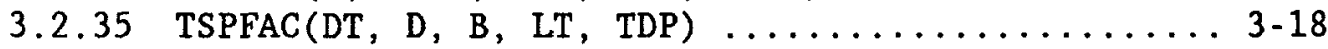

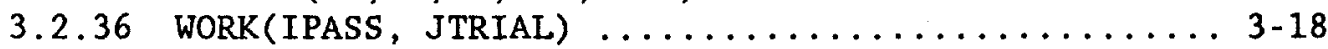

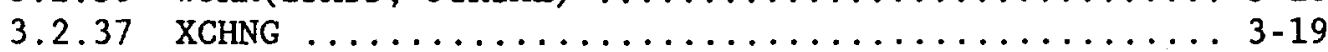

3.3 Parameter statements ........................ 3-19

3.4 Common Blocks .............................. 3-20

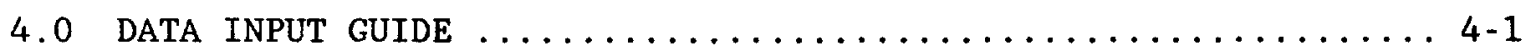

4.1 Execution Procedure for NEFTRAN II $\ldots \ldots \ldots \ldots \ldots \ldots \ldots$ 4-1

4.2 General Input Description ................... 4-2

4.3 Parameter Input Description .................. 4-3

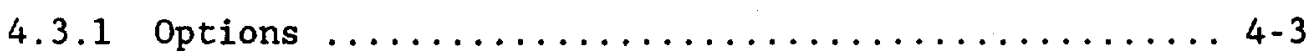

4.3.2 Problem Size Parameters ................. 4-10 
4.3.3 Source and Flow Parameters $\ldots \ldots \ldots \ldots \ldots \ldots \ldots$ 4-12

4.3.4 Time Parameters ........................ 4-14

4.3.5 Increment-Determination Parameters ........... 4-15

4.4 Array Input Description $\ldots \ldots \ldots \ldots \ldots \ldots \ldots \ldots \ldots \ldots \ldots$ 4-19

4.4.1 Network Leg Properties Array ............... 4-20

4.4.2 Junction Properties Array ................ 4-21

4.4.3 Migration Path Properties Array ............ 4-22

4.4.4 Decay Chain Array ...................... 4-24

4.4.5 Element Properties Array $\ldots \ldots \ldots \ldots \ldots \ldots \ldots \ldots \ldots$ 4-27

4.5 Data Required for Multiple Simulations ............. 4-29

4.5.1 Multiple Data Sets ................... 4-29

4.5 .2 Use of Subroutine GETRV ............... 4-31

4.6 Sample Input File...................... 4-31

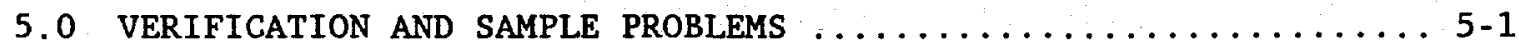

5.1 Sample Problem 1 - DVM and Analytical Transport Model.... 5-1

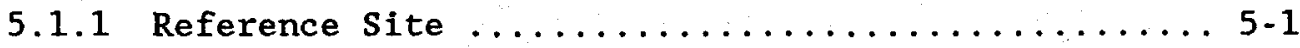

5.1 .2 Network Flow Model $\ldots \ldots \ldots \ldots \ldots \ldots \ldots \ldots \ldots \ldots \ldots .5$

5.1.3. Data Set \#1 ........................ 5-5

5.1 .4 Data Set \#2 ......................... 5-19

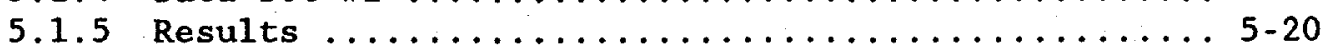

5.2 Sample Problem 2 - Matrix Diffusion ............... 5-20

5.3 Sample Problem 3 - Time-dependent DVM Velocities ....... 5-29

6.0 REFERENCES ........................... 6 .

APPENDIX A Species Velocity Model................... A-1

APPENDIX B Discharge Mode1......................

APPENDIX C Distribution of Source Into Migration Path

Using Mixing-Cell Model...................

APPENDIX D Storage of Decay Chain Information,........... D-1

APPENDIX E Output Files for Sample Problems.............. E-1 
2.1. Illustration of Conservation of Mass at a Junction........ 2-2

2.2. Example Flow Network for Use in NEFTRAN II ............ 2-6

2.3. The Mixing Cell ........................... 2-17

2.4. The Spatial Green's Function .................... 2-24

2.5 Numerical Simulation of the Transport of an Instantaneous Point Source.............................. 2-26

2.6. Illustration of the Mixing Fraction Concept ........... 2-29

2.7. Example Breakthrough Curves (NEFTRAN II,

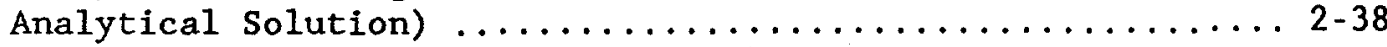

2.8. Example Breakthrough Curves with Concentration on a Logarithmic Scale ...................... 2-40

2.9. Comparison of Finite Difference Solution to Analytical Solution ................................. 2-41

2.10. Discharge Rate Curves for Problem Simulation Time $=$

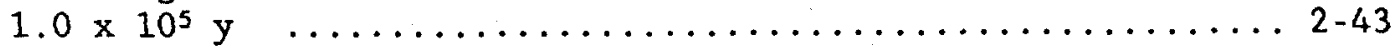

2.11 Discharge Rate Curves for Problem Simulation Time =

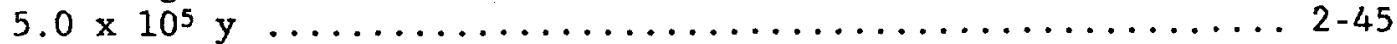

2.12. Discharge Rate Curves for Problem Simulation Time $=$ $1.0 \times 10^{6}$ y.................................... 2-46

2.13. Illustration of Four Possible Situations Arising Because of an Overlap of a Packet and a Given Receiver Block ..... 2-53

2.14. Illustration of Catcher Blocks Appended to Leg L ........ 2-55

2.15. Illustration of Adjacent System of Grid Blocks Simulating the Rock Matrix in Dual-Porosity Legs ............. 2-57

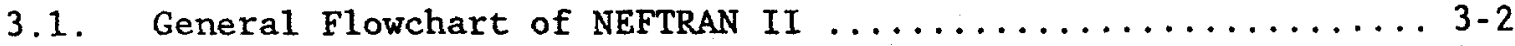

3.2. Flowchart of Source Module in NEFTRAN II $\ldots \ldots \ldots \ldots \ldots \ldots \ldots .4 .4$

3.3. Flowchart of Transport Module in NEFTRAN II $\ldots \ldots \ldots \ldots \ldots \ldots$ 3-5

5.1. Physiographic Setting of the Reference Site ........... 5-2

5.2. Reference Site Geology ....................... 5-3

5.3. Ground-Water Flow at Reference Site ............... 5-4

5.4. Hydraulic Head Distribution at the Reference Site ........ 5-6

5.5. SWIFT II Gridding for the Reference Site ............ 5-7

5.6. NEFTRAN II Flow Network Superimposed on SWIFT II Grid

(Dark Lines Indicate Network Legs) ................ 5-8

5.7. NEFTRAN II Flow Network Used in Sample Problem \#1 ........ 5-9

5.8. ${ }^{237} \mathrm{~Np}$ Discharge Rates for Sample Problem \#1 ........... 5-21

5.9. 233U Discharge Rates for Sample Problem \#1 ............ 5-22

5.10. ${ }^{229} \mathrm{Th}$ Discharge Rates for Sample Problem \#1 ........... 5-23

5.11. Breakthrough Curve for Sample Problem \#2 ............ 5-30

5.12. Discharge Rate Curves for Sample Problem $\# 3 \ldots \ldots \ldots \ldots \ldots \ldots$ 5-35 
B.1 Total and Partial Discharge of a Typical Velocity Packet $\ldots \ldots \ldots \ldots \ldots \ldots \ldots \ldots \ldots \ldots \ldots \ldots \ldots \ldots \ldots . \ldots \ldots$ B 2

C.1 The Grid Block Numbering Convention................

C.2 Shaded Area Represents the Region of Integration for Total Source of Species 2 from Species $1 \ldots \ldots \ldots \ldots \ldots \ldots$. 6 . 6

C.3 Possible Integration Regions for the Case $v_{1}-v_{2} \ldots \ldots \ldots$ C-8

C.4 The Possible Regions of Integration for Subcase $2.1 \ldots \ldots \ldots c-11$

C.5 Regions of Integration for Subcase $2.2 \ldots \ldots \ldots \ldots \ldots \ldots \ldots$ C-14

C. 6 Regions of Integration for Subcase $2.3 \ldots \ldots \ldots \ldots \ldots \ldots$ 
2.1. Boundary and Initial Conditions for the Analytical

Solution Transport Model ...................... 2-9

2.2. Parameters for Example Problem.................. 2-42

4.1. Input Template for Standard Print Options............. 4-4

4.2. Input Template for External File Options............. 4-7

4.3. Input Template for Run Control Options.............. 4-8

4.4. Input Template for Debug Print Options.................4-10

4.5. Input Template for Problem Size Parameters.............. 4-12

4.6. Input Template for Source/Flow Parameters.............. 4-14

4.7. Input Template for Time Parameters................ 4-15

4.8. Input Template For Increment Determination Parameters...... 4-18

4.9. Recommended Sets of Values for the Increment-

Determination Parameters ...................... 4-19

4.10. Input Template for Network Leg Properties Array......... 4-21

4.11. Input Template for Network Junction Properties Array...... 4-22

4.12. Input Template for Migration Path Properties Array........ 4-23

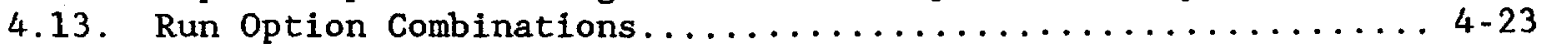

4.14. Migration Path Array Parameters as a Function of

Run Option............................. 4-24

4.15. Input Template for Decay Chains Array............... 4-28

4.16. Input Template for Element Properties Array............ 4-29

4.17. Input Template for Multiple Data Set Options.......... 4-30

4.18. Sample Input File......................... 42

5.1. Input File for Sample Problem \#1 ................ 5-10

5.2. Reference Site Hydraulic Properties ................ $5-17$

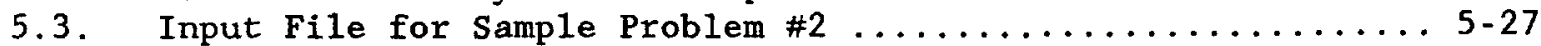

5.4. Input File for Sample Problem \#3 ................. 5-31

5.5. External File of Time-Varying Velocity Data ........... 5-33

C.1 Integration Limits Needed to Evaluate Equation C.14

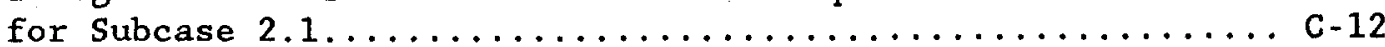

C.2 Integration Limits Needed to Evaluate Equation C.14

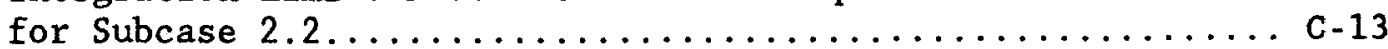

C.3 Integration Limits Needed to Evaluate Equation C.14

for Subcase $2.3 \ldots \ldots \ldots \ldots \ldots \ldots \ldots \ldots \ldots \ldots \ldots \ldots \ldots \ldots$ 


\section{ACKNOWLEDGMENT}

The authors wish to express their thanks to David Gallegos, Phil Pohl, Ron DyKhuizen and Tim McCartin for their review of this document, and to Ms. Irene Gonzales for her hard work in typing this document. They would also like to thank Dr. Evaristo Bonano for his support and consultation on the development of NEFTRAN II and for reviewing this document. 
This report describes the NEFTRAN II (NEtwork Flow and TRANsport in TimeDependent Velocity Fields) computer code developed by Sandia National Laboratories (SNL) for the U.S. Nuclear Regulatory Commission (NRC). The following sections discuss pertinent background concerning the NEFTRAN II computer code, the purpose of developing the code, and the structure of this report.

\subsection{Background}

NEFTRAN II is the fourth in a family of codes developed by SNL for the NRC. Predecessors to NEFTRAN II are NEFTRAN (Longsine and others, 1987), NWFT/DVM (Campbel1 and others, 1981b), and NWFT (Campbel1 and others, 1979). These codes have evolved such that each new version contains all the capabilities of its immediate predecessor in addition to new features. NWFT and NWFT/DVM were developed as a tool to help the NRC assess the performance of high-level radioactive waste disposal sites in geologic media. Specifically, NWFT and NWFT/DVM were used to simulate radionuclide ground-water transport through saturated porous media from a repository to a discharge point. With NEFTRAN, the capability to simulate transport through saturated dual-porosity or fractured media was added. In NEFTRAN II, the capability to transport radionuclides through time-dependent velocity fields, which may be applied to simulate transport in unsaturated media, has been added. NWFT changed as (1) the sites considered for HLW disposal changed, (2) the analyses required to apply for licensing and assessing a license evolved, and (3) the standards for HLW disposal and analyses required to prove compliance with those standards evolved. Although NEFTRAN II is the fourth-generation code, this document is a complete description of the code and reference to past user's manuals should not be necessary.

\section{$1.2 \quad$ Purpose}

The purpose of the NEFTRAN II computer code is to simulate radionuclide transport in ground water as part of a performance assessment methodology for a high-level radioactive waste repository. In the performance assessment methodology, a large number of simulations are required to address parameter uncertainty and sensitivity. Furthermore, since the performance measure for a HLW repository in the U.S. is integrated discharge to the accessible environment at 10000 years, relatively long simulation times are required. Consequently, the radionuclide transport code for use in a performance assessment methodology must be computationally efficient, as well as being able to simulate the transport of multiple radioactive chains with multiple members. NEFTRAN II and its predecessors were developed to meet these performance assessment requirements.

There are several ways in which NEFTRAN II can be used in a performance assessment methodology. For a saturated, steady-state flow field, the network flow model contained in NEFTRAN II can be used in conjunction with a detailed flow model such as that contained in the SWIFT II code (Reeves and others, 1986a, 1986b). The mathematical models in the SWIFT II code are based on the assumption that the medium in which flow 
and transport are occurring is continuous, which may lead to a fully multi-dimensional flow system. The flow and transport models contained in NEFTRAN II are based on the assumption that all significant flow and radionuclide transport takes place along pathways that can be simulated as discrete one-dimensional legs or paths. These legs are assembled to form a multi-dimensional network representation of the flow field. To define this network representation and the migration path, the results of a detailed flow model (such as the one contained in SWIFT II), and a particle-tracking method (such as the one used by Bonano and others, 1989) can be used. The particle-tracking method is used to define the trajectory that a tracer particle released from a given point (e.g., the repository) will follow until it crosses some prescribed boundary (e.g., the accessible environment) in the flow field predicted by SWIFT II. Based on the particle tracking model and the flow field predicted by SWIFT II the length, location of end points, cross-sectional area, migration path, and all hydraulic properties of each leg in the network flow system can be defined. With the appropriate boundary pressures from SWIFT II, the network can reproduce the flow field. As long as this reproduction remains valid under statistical variation, the use of a network to define the flow field makes NEFTRAN II a more computationally efficient code than codes like SWIFT II and therefore, for the multiple simulations required for performance assessment, using NEFTRAN II is more practical.

NEFTRAN II can also be used in a performance assessment methodology without utilizing the network flow model (i.e., without modeling flow), by direct input of pore velocities into the transport model. In this case NEFTRAN II can be coupled with a flow model such as the one contained in DCM3D (Updegraff and Lee, 1991), which simulates flow in unsaturated, fractured media. Again, a particle tracking-method would be used to determine a dominant one-dimensional transport path for NEFTRAN II from the results of DCM $3 D$. Significant changes in pore velocities and saturations with time along this path can also be determined from a detailed flow model such as the one contained in DCM3D and then utilized by NEFTRAN II for the radionuclide transport analysis. The underlying assumption is that the flow path is essentially constant with time and only the magnitude of the velocity is time-dependent.

Although NEFTRAN II is designed for a performance assessment methodology, it can be used for general analysis of radionuclide transport in ground water and can be run easily by a wide variety of users. NEFTRAN II may appear extremely simple to use, but applying it to a problem, and the meaning of the results obtained, requires as much thought and analysis as using what might appear to be a more complex code. For this reason, the user must have knowledge of flow and transport through porous media and must be able to discern when the models contained in NEFTRAN II are adequate for the problem being simulated. The purpose of this report is not to tell the user when to use NEFTRAN II, but rather how to use NEFTRAN II once the user has decided the models that it contains are appropriate for the specific problem being simulated. 


\subsection{Report Structure}

The structure of this report is as follows. Chapter 2 describes the models contained in NEFTRAN II, which includes the network flow model, the analytical transport model, the source model, the distributed velocity method (DVM) transport model, and the matrix diffusion model. Chapter 3 describes the code structure, the parameter statements used in the code, the common blocks used in the code, and the subroutines contained in the code. Chapter 4 is a data input guide, and verification and sample problems are presented in Chapter 5. For the first time user, it is recommended that Chapters 2, the first section of Chapter 3 , Chapter 4 and Chapter 5 be read initially, since the last three sections of Chapter 3 and the appendices are a more detailed description of the actual code and may be of more utility as the user gains experience with the code. Special attention should be paid to sections 2.4 .3 and 2.4.4 which describe the criteria for space- and time-increment determination. The numerical criteria for the DVM numerical technique used in NEFTRAN II are substantially different from other numerical transport schemes and must be understood before the code can be used properly. 


\section{0}

The following sections describe the flow, source, and transport models contained in NEFTRAN II. The theoretical basis for each model, as well as numerical implementation, is discussed.

The first step is to define the ground-water flow path and, if necessary, the ground-water flow rate through the source region. These can be defined in one of three ways:

- specify the geometric and hydraulic properties of a flow network in the standard NEFTRAN II input file (described in Section 4) and use the network flow model (described in section 2.1) to solve for the pressures, flow rates and velocities,

- input pore velocities and source flow rates via the standard NEFTRAN II input file, or

- input pore velocities and source flow rates from external files (described in Section 2.4.11 and Section 2.3.2). This option was designed to allow for time-dependent flow behavior when simulating radionuclide transport.

After defining the ground-water flow path, the next step is to simulate radionuclide transport.

Section 2.2 contains the theory and implementation for an analytical solution to the convective-dispersion transport equation. This solution is restricted to (1) decay chains having no more than three members, (2) each member of the decay chain must have identical retardation factors, (3) a steady-state flow field, and (4) the source must be equivalent to a concentration boundary condition. This analytical solution, coupled with a network flow model (fixed number of legs and junctions), comprised the NWFT model (Campbe11, and others, 1979).

Introduction of the distributed velocity method (DVM) (Campbe11 and others, 1980) for radionuclide transport into NWFT resulted in the second code of the sequence, NWFT/DVM (Campbe11 and others, 1981a, 1981b). With DVM, many limitations imposed by use of the analytical solution were eliminated by allowing the consideration of radionuclide chains of arbitrary length, the members of which could have significantly different retardation factors. Most of the capabilities of NWFT/DVM, NEFTRAN (Longsine and others, 1987) and NEFTRAN II, center around the DVM technique. For example, the source model (described in Section 2.3) was developed specifically for DVM and the matrix diffusion model (Section $2.4 .10)$ is directly coupled to DVM. The capability to transport radionuclides in time-dependent velocity fields (discussed in Section 2.4.11) is also directly coupled to the DVM transport model. None of these models can be used with the analytical transport solution. 


\subsection{Network Flow Mode1}

\subsubsection{Theory}

In the flow-network model, the driving force for flow through a leg is a pressure gradient. Consequently, the network flow model contained in NEFTRAN II can be used to simulate saturated ground-water flow. The flow network is used to represent a saturated flow system by solving the conservation-of-mass equation at each junction. This principle can be expressed mathematically as

$$
\Sigma_{\ell} M_{\ell}=0
$$

where the sum is over all legs connected at the given junction, and $M$ is the mass flow rate for the $l^{\text {th }}$ leg in units of mass per unit time. Flow rates into the junction are considered positive and flow rates out of the junction are considered negative. Figure 2.1 illustrates this principle for three legs surrounding a junction.

For this simple example, Equation 2.1 becomes

$$
M_{1}+M_{2}-M_{3}=0
$$

or

$$
M_{1}+M_{2}=M_{3}
$$

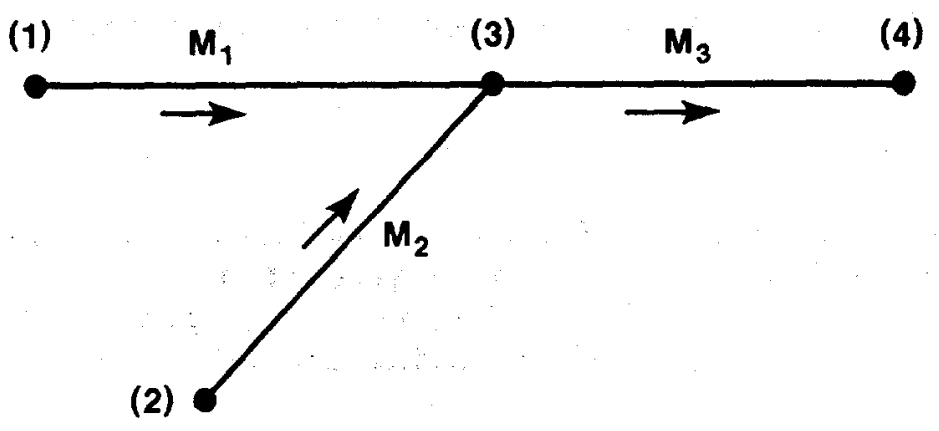

Figure 2.1. Illustration of Conservation of Mass at a Junction 
If the $\ell^{\text {th }} \operatorname{leg}$ is bounded by Junctions $\ell 1$ and $\ell 2$, the mass flow rate in the leg is defined by

$$
M_{\ell}=\rho_{\ell} K_{\ell} A_{\ell}\left[\frac{\left(P_{\ell 1}-P_{\ell 2}\right)}{Z_{\ell} P_{\ell} g}+\frac{\left(E_{\ell 1}-E_{\ell 2}\right)}{Z_{\ell}}\right],
$$

where $A_{f}$ is cross-sectional area $\left(\right.$ length $\left.^{2}\right), K_{\ell}$ is hydraulic conductivity (length/time), $E_{\text {is }}$ is elevation of the $i^{\text {th }}$ junction, $g$ is the acceleration of gravity (length/time ${ }^{2}$ ), $P_{l i}$ is pressure (force/area) at

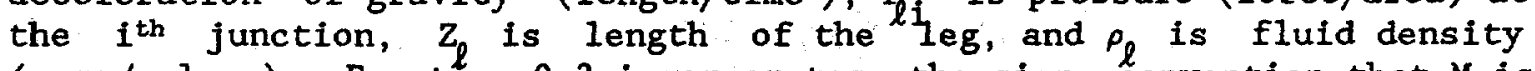
(mass/volume). Equation 2.3 incorporates the sign convention that $M_{\text {is }}$ positive if the flow direction is from Junction $\ell 1$ to Junction 22 . The hydraulic conductivity is weighted as

$$
K_{\ell}=K_{\ell}^{\prime}\left(\frac{\mu_{f}}{\mu_{\ell}}\right)\left(\frac{\rho_{\ell}}{\rho_{f}}\right)
$$

to account for the effects of brine concentration on the flow; $K_{\ell}^{\prime}$ is the fresh-water hydraulic conductivity for the $\ell^{\text {th }} l e g, \mu_{f}$ and $\rho$ the viscosity (mass/length/time) and density of fresh water at approximately $20^{\circ} \mathrm{C}$, and $\mu_{\ell}$ and $\rho_{\ell}$ are the actual viscosity and density of the brine solution in the $\ell^{\text {th }}$ leg. The manner in which $\mu_{l}$ and $\rho_{l}$ are determined in NEFTRAN II is discussed in Section 2.1.3.

Equation 2.3 is a mathematical statement of Darcy's law in which the total head difference is given by the sum of dynamic pressure and elevation differences. This equation typically has three unknowns $-\mathrm{M}_{\ell}, \mathrm{P}_{\ell^{1}}$, and $\mathrm{P}_{\ell^{2}}$, unless either Junction $\ell 1$ or Junction $\ell 2$ has been specified as a boundary junction with known pressure. For each junction in the network, Equation 2.1 is written and Equation 2.3 is substituted for $M_{f}$. This eliminates the mass flow rates as unknowns leaving only the junction pressures to be determined since the elevations are supplied as part of input.

\subsubsection{Implementation}

Based on the above discussion, NEFTRAN II implements the network flow model by applying Equation 2.1 to a generalized flow network system. For example, if Figure 2.1 is part of a larger network, applying Equation 2.1 to Junction 3 in Figure 2.1, and substituting Equation 2.3 for $\mathrm{M}_{\ell}$ gives

$$
\begin{aligned}
& \theta_{1} P_{1}+\theta_{2} P_{2}-\left(\theta_{1}+\theta_{2}+\theta_{3}\right) P_{3}+\theta_{3} P_{4} \\
= & -\theta_{1} \rho_{1} g\left(E_{1}-E_{3}\right)-\theta_{2} \rho_{2} g\left(E_{2}-E_{3}\right)+\theta_{3} \rho_{3} g\left(E_{3}-E_{4}\right),
\end{aligned}
$$

where $\theta_{\ell}=A_{f} K_{l} / Z_{l} g$. Repeating this process for every junction in the network results in a matrix equation of the form, 


$$
\left[\begin{array}{cccc}
\ddots & & & \\
\cdots \theta_{1} \theta_{2}-\left(\theta_{1}+\theta_{2}+\theta_{3}\right) & \theta_{4} \\
& & \ddots &
\end{array}\right]\left[\begin{array}{l}
\vdots \\
\mathrm{P}_{1} \\
\mathrm{p}_{2} \\
\mathrm{p}_{3}^{3} \\
\mathrm{P}_{4}
\end{array}\right]\left[\begin{array}{l} 
\\
\vdots \\
\mathrm{e}_{3} \\
\vdots
\end{array}\right]
$$

which can be written using matrix notation as

$$
\underset{\theta}{\theta}=\underline{e},
$$

where $\underline{\theta}$ is the matrix of coefficients, $p$ the vector of unknown pressures, and $\underline{e}$ the vector of junction elevations and boundary pressures representing the right-hand side of Equation 2.5. The dimension of the matrix equation is the number of junctions having unknown pressure.

After solving for the junction pressures, NEFTRAN II calculates the mass flow rate in each leg using Equation 2.3 and divides by the corresponding density to determine the volumetric flow rate. The volumetric flow rate is used to obtain the average fluid velocity for each leg along the radionuclide migration path used by the transport models in NEFTRAN II. Specifically, from Equation 2.3,

$$
\mathrm{Q}_{\ell}=\mathrm{K}_{\ell^{\mathrm{A}} \ell}\left[\frac{\left(\mathrm{P}_{\ell 1}-\mathrm{P}_{\ell 2}\right)}{\mathrm{Z}_{\ell}{ }^{\rho_{\ell}} \mathrm{g}}+\frac{\left(\mathrm{E}_{\ell 1}-\mathrm{E}_{\ell 2}\right)}{\mathrm{Z}_{\ell}}\right] \text {, }
$$

where $Q_{\ell}$ is the volumetric flow rate (volume/time) in the $\ell^{\text {th }}$ leg. The average fluid pore velocity (length/time) in the leg is

$$
\mathrm{v}_{\ell}=\frac{\mathrm{Q}_{\ell}}{\mathrm{A}_{\ell} \phi_{\ell}}
$$

where $\phi_{\ell}$ is the effective porosity (dimensionless).

In NEFTRAN II, Equation 2.7 is solved using a special case of the Gaussian elimination technique designed for banded matrices. For efficiency, the user should keep the band width as small as possible. Except for boundary junctions with known pressures, this means that the junction numbers surrounding a given junction should be close to the given junction number. In fact, if Junctions $\ell 1$ and $\ell 2$ are connected by $a$ dimensioning statements in the code require $|\ell 1-\ell 2| \leq 5$. 
The network flow model in NEFTRAN II allows the user to represent any flow network by specifying the hydraulic properties of each network leg, the junctions bounding the leg, junction elevations, and the known pressures at the boundary junctions. Figure 2.2 shows an example flow network for use in NEFTRAN II to simulate saturated ground-water flow. Horizontal legs might represent different geological strata while vertical legs could represent potential pathways for hydraulic communication between layers. In this example, the user would have to specify pressures at Junctions 1,2 , and 3 , as these are boundaries in the flow network.

\subsubsection{Fluid Density and Viscosity Submodels}

Fluid density and viscosity $\left(\rho_{\ell}\right.$ and $\mu_{\ell}$ ) are needed for the network flow model, as defined in Equations 2.3 and 2.4. In reality, water density and viscosity are functions of temperature, pressure, and brine concentration. However, the dependence of water density and viscosity on pressure has been found to be negligible (Cranwell and others, 1982). Furthermore, NEFTRAN II was designed to simulate transport processes under isothermal conditions. Thus, water density is assumed to be a function of brine concentration only. Specifically, water density is assumed to be a linear function of brine concentration; i.e.,

$$
\rho(C)=\rho_{f}+C\left[\rho_{s}-\rho_{f}\right],
$$

where $C$ is the dimensionless brine concentration ( $C=0$ for fresh water and $C=1$ for saturated brine), $\rho_{f}$ the density of fresh water at the reference temperature $\left(62.31 \mathrm{~b} / \mathrm{ft}^{3}\right.$ at $\left.68^{\circ} \mathrm{F}\right)$, and $\rho_{\mathrm{s}}$ the density of saturated brine at the reference temperature $\left(74.021 \mathrm{~b} / \mathrm{ft}^{3}\right.$ at $\left.68^{\circ} \mathrm{F}\right)$. Equation 2.10 is used to determine the fluid density for each network leg based on the relative brine concentration (C) of each leg.

The dependence of viscosity on concentration of dissolved solids can be approximated as

$$
\mu(c)=\mu(0)\left[1.0+0.005 \sqrt{\left.\sum_{i=1}^{N} c_{i}+\sum_{i=1}^{N} A_{i} c_{i}\right],}\right.
$$

where $c_{i}$ is the concentration of dissolved ion (i) in moles/liter, $A_{i}$ the temperature dependent coefficient, $\mu(0)$ the viscosity of fresh water at $68^{\circ} \mathrm{F}$, and $\mathrm{N}$ the number of dissolved species.

For ground-water systems, solutions containing more than 10 or 15 moles/liter of dissolved ions are unlikely; therefore, the square root term in Equation 2.11 can be neglected. Assuming $\mathrm{NaCl}$ is the primary dissolved mineral, then $\mathrm{N}=2$ and the dissolved species are $\mathrm{Na}^{+}$and $\mathrm{Cl}^{-}$. 


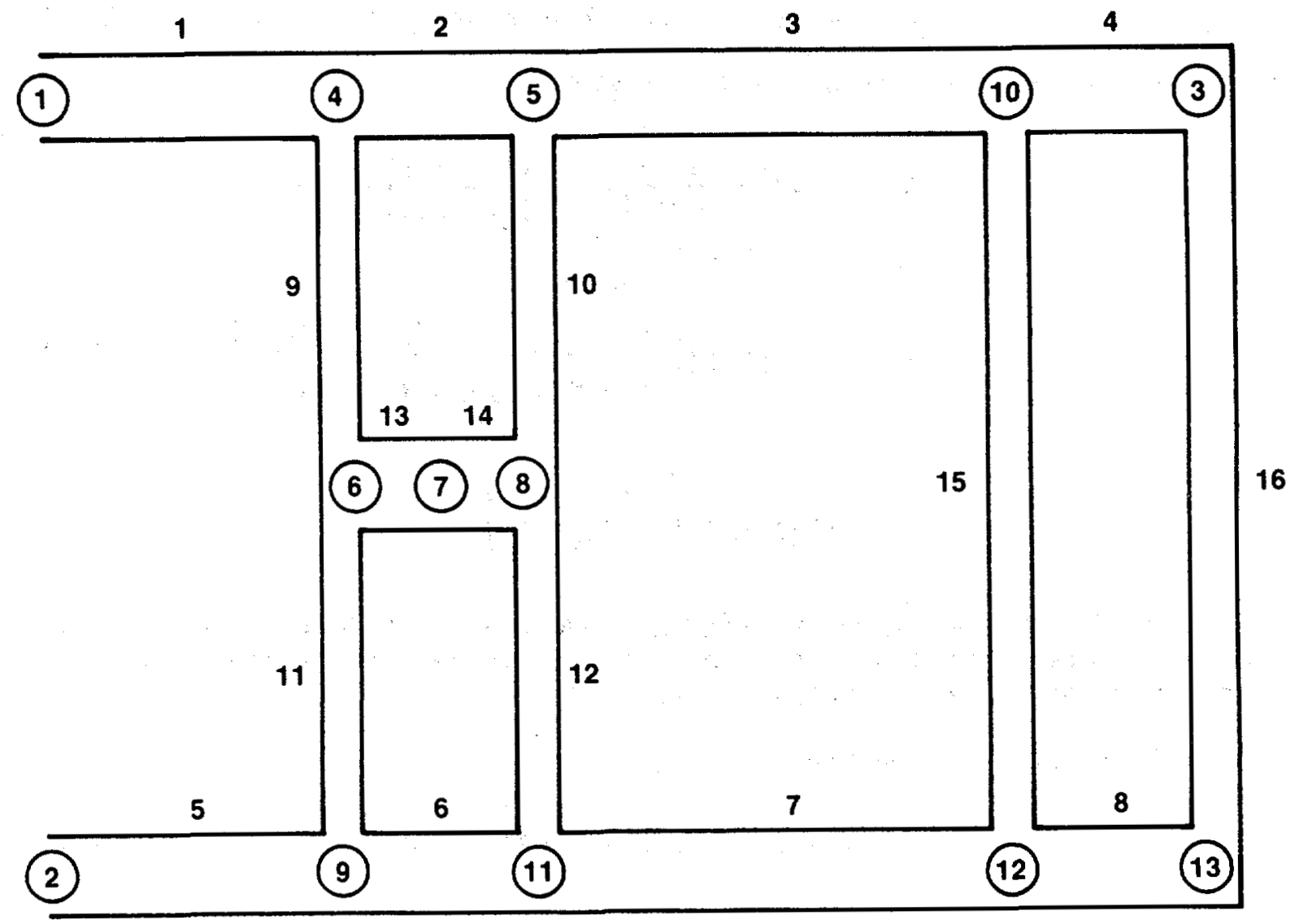

Figure 2.2. Example Flow Network for Use in NEFTRAN II 
For these ions, the coefficients (A) can be assumed to be independent of temperature, and are defined as

$$
\mathrm{A}\left(\mathrm{Cl}^{-}\right)=0
$$

and

$$
\mathrm{A}\left(\mathrm{Na}^{+}\right)=0.08
$$

Given these arguments, Equation 2.11 can be simplified to

$$
\mu(\mathrm{C})=\mu(0)\left[1.0+0.08 \mathrm{c}\left(\mathrm{Na}^{+}\right)\right] .
$$

The concentration of dissolved $\mathrm{Na}^{+}$is given as

$$
\begin{aligned}
c\left(\mathrm{Na}^{+}\right) & =\mathrm{w}\left(\frac{\mathrm{lb} \mathrm{salt}}{\mathrm{lb} \mathrm{sol}}\right) \cdot \mathrm{c} \cdot \rho\left(\frac{\mathrm{lb} \mathrm{salt}}{\mathrm{ft}^{3} \mathrm{sol}}\right) \\
& \cdot 454\left(\frac{\mathrm{gm}}{\mathrm{Ib}}\right) \cdot\left(\frac{1}{2.83 \times 10^{4}}\right)\left(\frac{\mathrm{ft}^{3}}{\mathrm{~cm}^{3}}\right) 1000\left(\frac{\mathrm{cm}^{3}}{\ell}\right) \\
& \cdot\left(\frac{1}{58.443}\right)\left(\frac{\text { moles NaCl}}{\mathrm{gm}}\right)
\end{aligned}
$$

where $W$ is the weight fraction of salt in saturated brine, $C$ the dimensionless brine concentration, and $\rho(-\rho(C))$ the density of brine at concentration C (from Equation 2.10).

Thus, Equation 2.12 becomes

$$
\mu(C)=\mu(0)[1.0+0.0219 W C \rho]
$$

Equation 2.14 is used in NEFTRAN II to estimate fluid viscosity for each leg in the flow network based on the relative brine concentration (C) within the leg.

\subsection{Analytical Transport Model}

The analytical transport model that formed the basis for NWFT has been retained in each of its successors. The theory and implementation for this model is discussed in the following subsections. 


\subsubsection{Theory}

NEFTRAN II contains two models that can simulate radionuclide transport. The analytical transport option simulates the migration of a three-member radioactive decay chain in a one-dimensional constant velocity system, as described by the following differential equations:

$$
\begin{gathered}
\mathrm{R} \frac{\partial \mathrm{N}_{1}}{\partial t}=\mathrm{D} \frac{\partial^{2} \mathrm{~N}_{1}}{\partial \mathrm{X}^{2}}-\mathrm{v} \frac{\partial \mathrm{N}_{1}}{\partial \mathrm{X}}-\mathrm{R} \lambda_{1} \mathrm{~N} \lambda_{1} \\
\mathrm{R} \frac{\partial \mathrm{N}_{2}}{\partial \mathrm{t}}=\mathrm{D} \frac{\partial^{2} \mathrm{~N}_{2}}{\partial \mathrm{X}^{2}}-\mathrm{v} \frac{\partial \mathrm{N}_{2}}{\partial \mathrm{X}}-\mathrm{R} \lambda_{2} \mathrm{~N}_{2}+\mathrm{R} \lambda_{1} \mathrm{~N}_{1} \\
\mathrm{R} \frac{\partial \mathrm{N}_{3}}{\partial t}=\mathrm{D} \frac{\partial^{2} \mathrm{~N}_{3}}{\partial \mathrm{X}^{2}}-\mathrm{v} \frac{\partial \mathrm{N}_{3}}{\partial \mathrm{X}}-\mathrm{R} \lambda_{3} \mathrm{~N}_{3}+\mathrm{R} \lambda_{2} \mathrm{~N}_{2},
\end{gathered}
$$

where $R$ is the constant retardation factor (dimensionless) that is assumed to be equal for all three radionuclides, $N_{i}$ the concentration (mass/volume) in solution of species $i, D(=|v| \alpha)$ the dispersion coefficient (length ${ }^{2} /$ time), $\alpha$ the dispersivity (length), $\lambda_{i}\left(=\ln 2 / \mathrm{T}_{1 / 2}\right.$ ) the decay constant for species $i\left(t i m e^{-1}\right)$, and $T_{1 / 2}$ the half-life for species i (time).

Equations 2.15 - 2.17 represent conservation of mass for a species in a differential volume. The left-hand side (LHS) in each of these equations denotes the rate of change of dissolved species within this volume. The terms in the right-hand side (RHS) represent the flux of species in and out of the volume, and the source/sink terms within the volume. Specifically, the first and second terms in the RHS stand for the net flux of the species by mechanical dispersion and convection, respectively while the last two terms represent production and depletion of the species due to radioactive decay.

The boundary conditions and initial condition used in conjunction with Equations 2.15, 2.16, and 2.17 are listed in Table 2.1. Based on these conditions, the source for the analytical transport model is a concentration boundary condition and the source model discussed in Section 2.3 is not implemented when the user selects the analytical transport model.

\subsubsection{Implementation}

To implement the analytical solution transport model in NEFTRAN II, the analytical solution for Equations $2.15,2.16$ and 2.17 with the indicated initial and boundary conditions is utilized. The time-dependent discharge rates predicted by Equations $2.15,2.16$, and 2.17 , as given by Lester, Jansen, and Burkholder (1975), are 
I. $\quad \frac{t=0, \text { all } x}{C_{1}=C_{2}=C_{3}}=\ldots=0$

II. $\quad \underline{0<t<\tau, x=0}$

$$
\begin{aligned}
& C_{1}=\frac{N_{1}(0)}{Q \tau} e^{-\lambda_{1} t} \\
& C_{2}=\frac{N_{2}(0)}{Q \tau} e^{-\lambda_{2} t}+\frac{\lambda_{1}}{\lambda_{2}-\lambda_{1}} \frac{N_{1}(0)}{Q \tau}\left(e^{-\lambda_{1} t}-e^{-\lambda_{2} t}\right) \\
& C_{3}=\frac{\mathrm{N}_{3}(0)}{\mathrm{Q} \tau} e^{-\lambda_{3} t}+\frac{\lambda_{2}}{\lambda_{3}-\lambda_{2}} \frac{\mathrm{N}_{2}(0)}{\mathrm{Q} \tau}\left(\mathrm{e}^{-\lambda_{2} t}-\mathrm{e}^{-\lambda_{3} t}\right) \\
& +\lambda_{1} \lambda_{2} \frac{\mathrm{N}_{1}(0)}{\mathrm{Q} \tau} \quad \frac{\mathrm{e}^{-\lambda_{1} t}}{\left(\lambda_{2}{ }^{\left.-\lambda_{1}\right)\left(\lambda_{3}-\lambda_{1}\right)}\right.}+\frac{\mathrm{e}^{-\lambda_{2} t}}{\left(\lambda_{1}-\lambda_{2}\right)\left(\lambda_{3}-\lambda_{2}\right)}+ \\
& \frac{e^{-\lambda_{3} t}}{\left(\lambda_{1}-\lambda_{3}\right)\left(\lambda_{2}-\lambda_{3}\right)}
\end{aligned}
$$

III. $\frac{t>\tau, x=0}{C_{1}=C_{2}=C_{3}}=\ldots=0$

IV. $\quad \frac{t>0, x \rightarrow \infty}{C_{1}, C_{2}, C_{3}, \ldots=\text { finite }}$

$\dot{\mathrm{N}}_{i}(0)$ is the initial inventory of species 1 , $Q$ the fluid flow rate, and $\tau$ the leach time for a constant leach rate source. 


$$
\begin{aligned}
& D_{1}(t)=\frac{N_{1}(0)}{2 \tau} e^{-\lambda_{1} t}[(U(t)-U(t-r) H(t-\tau))] \\
& D_{2}(t)=\left[\frac{N_{2}(0) e^{-\lambda_{2} t}}{2 r}+\frac{N_{1}(0)}{2 r}\left(\frac{\lambda_{1}}{\lambda_{2}-\lambda_{1}}\right)\left(e^{-\lambda_{1} t}-e^{-\lambda_{2} t}\right)\right] . \\
& {[[U(t)-U(t-r) H(t-\tau)]]} \\
& D_{3}(t)=\left[\frac{N_{3}(0)}{2 \tau} e^{-\lambda_{3} t}+\frac{N_{2}(0)}{2 \tau}\left(\frac{\lambda_{2}}{\lambda_{3}-\lambda_{2}}\right)\left(e^{-\lambda_{2} t}-e^{-\lambda_{3} t}\right)\right. \\
& +\frac{N_{1}(0)}{2 \tau} \lambda_{1} \lambda_{2} \frac{e^{-\lambda_{1} t}}{\left(\lambda_{2}-\lambda_{1}\right)\left(\lambda_{3}-\lambda_{1}\right)}+\frac{e^{-\lambda_{2} t}}{\left(\lambda_{1}-\lambda_{2}\right)\left(\lambda_{3}-\lambda_{2}\right)} \\
& \left.+\frac{e^{-\lambda_{3} t}}{\left(\lambda_{1}-\lambda_{3}\right)\left[\lambda_{2}-\lambda_{3}\right)}\right] \cdot[[U(t)-U(t-\tau) H(t-\tau)]] \text {, }
\end{aligned}
$$

where $\tau$ is the leach time for a constant leach rate source, and

$$
H(x)= \begin{cases}0 & x<0 \\ 1 & x \geq 0\end{cases}
$$

The function $U(t)$ in Equations $2.18-2.20$ is given by

$$
U(t)=\operatorname{erfc}\left[\frac{L_{P}-\frac{v}{R} t}{\sqrt{4 \alpha t v / R}}\right]+\exp \left[\frac{L_{P}}{\alpha}\right] \operatorname{erfc}\left[\frac{L_{P}+\frac{v}{R} t}{\sqrt{4 \alpha v t / R}}\right],
$$

where $L_{p}$ is the total migration path length.

The above equations are implemented in NEFTRAN II by defining $L_{p}$ as the total distance from source to discharge (i.e, summing the length of all the legs in the migration path), and $v / R$ as the average isotope velocity ( $v_{\text {avg }}$ ) accounting for retardation. This average isotopic velocity (length/time) is found by preserving the total migration time; i.e., 


$$
T_{m}=\frac{\Sigma L_{i} R_{1}}{v_{1}},
$$

where $L_{1}, R_{1}$, and $v_{1}$ are the length, retardation factor and average velocity in the $1^{\text {th }}$ leg; thus $v_{\text {avg }}$ is

$$
v_{\text {avg }}=\frac{L_{p}}{T_{m}}
$$

The analytical transport model can only be used to simulate radionuclide transport problems under the following conditions: (1) leach-1imited source with a constant leach rate (which is equivalent to the concentration boundary condition given in Table 2.1), (2) chain of three or fewer radionuclides, (3) identical retardation factors for all radionuclides in each leg, and (4) constant fluid velocity. To reiterate, the source used in the analytical transport model is based on the concentration boundary condition given in Table 2.1, and does not utilize the source model contained in NEFTRAN II that is discussed in Section 2.3.

The only remaining point of implementation is the frequency of reporting discharge rates. NEFTRAN II determines a time range for "significant discharge", $T_{1} \leq T \leq T_{2}$, based on the values of $T_{1}$ and $T_{2}$ given by

$$
T_{1}=t_{M}-4 \sigma_{T}
$$

and

$$
\mathrm{T}_{2}=\mathrm{T}_{\mathrm{m}}+\tau+4 \sigma_{\mathrm{T}}
$$

respectively, where $\sigma_{\mathrm{I}}$ is the standard deviation in time defined by

$$
\sigma_{\mathrm{T}}=\frac{\sqrt{2 \alpha \mathrm{L}_{\mathrm{p}}}}{\mathrm{v}_{\mathrm{avg}}}
$$

$\mathbf{T}_{1}$ is small enough to capture the initial breakthrough portion of the discharge curve by subtracting four standard deviations in time from the mean migration time, while $T_{2}$ captures the trailing portion of the curve by accounting for the source duration plus the same spread. If the dispersivity is large, it is possible that $T_{1}$ is less than the initial source release time. In that case $T_{1}$ is replaced by the release time. Similarly, if $\mathrm{T}_{2}$ exceeds the problem simulation time, it is replaced by the problem simulation time. The resulting time range from $T_{1}$ to $T_{2}$ is divided by 100 to define a time increment for the analytical transport model, $\Delta t$, and discharge is reported 101 times (i.e., $T_{1}+i \Delta t, i=0$, $1, \ldots, 100$ ). 


\subsection{Source Mode1}

The source model in NEFTRAN II generates source rates for radionuclides exiting the repository and entering a migration path in the geosphere. As discussed above, the analytical transport model incorporates a concentration boundary condition. Therefore, the source model contained in NEFTRAN II is only implemented with the DVM transport model. The source model is executed independently of the DVM radionuclide-transport calculations in NEFTRAN II. Consequently, source rates generated by NEFTRAN II, in principle, could be used in other transport models. Conversely, it would be straightforward (though not currently implemented) to use source rates from other models as input for DVM transport calculations in NEFTRAN II.

The NEFTRAN II source model considers the fraction of the waste inventory in contact with circulating ground water, waste-form leaching, radionuclide solubilities, flow rates, the source area pore volume, and radioactive decay. A fixed delay time for the onset of leaching and/or migration can also be accounted for in the source model. Source model results are cast as time-dependent rates at which each radionuclide enters a migration path. The user provides the initial inventory of radionuclides in the repository as input. The type of radionuclide release scenario being simulated and the extent of the disruption will determine what fraction of the total emplaced waste is accessed by circulating ground water.

Leaching is assumed to be the primary mechanism for the release of radionuclides from the waste form (e.g., glass matrix). The leach rate will depend in part on the matrix material, fluid flow rates and ground-water chemistry. Because NRC contractors other than SNL were charged with developing adequate source term models, NEFTRAN II only implements two simple leach models. These are a constant leach rate and an exponential leach rate. In the constant-leach rate model, the amount of waste that leaches per unit time is a constant fraction of the initial mass present. In the exponential model, the amount of waste that leaches per unit time is a constant fraction of the remaining waste mass. The source model was designed so that other leach models can be easily incorporated when available data suggest a need.

Some radionuclides have been shown to be quite insoluble in ground water due to a low solubility of the radionuclides, a high leach rate from the waste form, and/or a low inflow rate of uncontaminated water. If any of these were the case, there could be more mass of radionuclides free of the waste matrix than can be dissolved in ground water. The NEFTRAN II source model accounts for this phenomenon by maintaining an inventory labeled "leached but undissolved." If such an inventory is necessary, it can act as a source of radionuclides even after all material has been leached from the waste matrix. Solubility limits can, therefore, have the conjunctive effects of lowering nuclide concentrations and extending the source pulse in time. 
Dilution caused by the influx of uncontaminated ground water can reduce contaminant concentrations exiting the repository. Dilution is accounted for by offering an option in NEFTRAN II to treat the repository as a mixing cell; that is, water entering the mixing cell is assumed to instantly, and uniformly, mix with any dissolved material in the cell. The source is then distributed into the transport path based on the diluted concentrations. The other option for distributing the source into the transport path in NEFTRAN II is the flow-through source model which distributes the source evenly into the source leg (the first leg in the migration path). The flow-through source model assumes that source material leaves the source volume as it dissolves and dilution by uncontaminated water does not take place.

A new feature in NEFTRAN II allows the flow rate through the repository to change with time. The source model integrates the time-varying flow rate to estimate the total inflow over a time step. Thus, the amount of radionuclide that can dissolve may also change with time. However, this is the only effect of time-varying flow rates since current implementation does not allow the mixing-cell model to be used in conjunction with time-varying flow rates.

As indicated above, there are several different options available in the NEFTRAN II source model. These options can be classified into two different sets. First, there are options related to how the radioactive isotopes become available for transport from the waste form (e.g, leachlimited, solubility-limited, or both). Second, there are options that determine how the repository is to be treated (e.g., mixing-cell source, flow-through source).

Radioactive decay causes the inventory to change with time. The inventory remaining in the waste form (i.e., unleached), the leached-butundissolved inventory, and radionuclide concentrations in water (i.e., dissolved) all decrease due to radioactive decay and potentially increase due to production. These effects are taken into account in the source model contained in NEFTRAN II.

\subsubsection{Theory}

Before describing the governing equations of the NEFTRAN II source model, the notation used to denote decay chains is discussed. For simplification, it is assumed that species 1 is the parent of species 2 is the parent of species 3 , etc. For simplicity this is written as $1 \rightarrow 2 \rightarrow 3 \ldots .$. . The underlying assumption is that smaller indexed species decay to larger indexed species and that there are no branches in the decay chain. In practice, NEFTRAN II can treat branching using branching fractions supplied by the user. To illustrate the notation, assume that there are $I$ isotopes in a decay chain indexed as described above. The inventory (atoms per mass of waste matrix) for species i is given by 


$$
\frac{d N_{1}}{d t}= \begin{cases}-\lambda_{1} N_{1} & \text { if } 1=1 \\ -\lambda_{1} N_{1}+\lambda_{1-1} N_{1-1} & , \text { if } 1<1 \leq 1\end{cases}
$$

where $N_{1}$ is the inventory of species 1 (atoms/mass of waste matrix), $\lambda_{1}\left(-\ln 2 / T_{1 / 2}\right)$ the decay constant for species $i$ (time $\left.e^{-1}\right)$, and $T_{1 / 2}$ the half-life for species i (time).

Equation 2.28 represent a system of equations that can be solved analytically, and the solutions are known as the Bateman equations. The form of the solution implemented in NEFTRAN II yields decay and production fractions over a time interval from zero to time $t$. For parent $p \leq i$ the fraction is given by

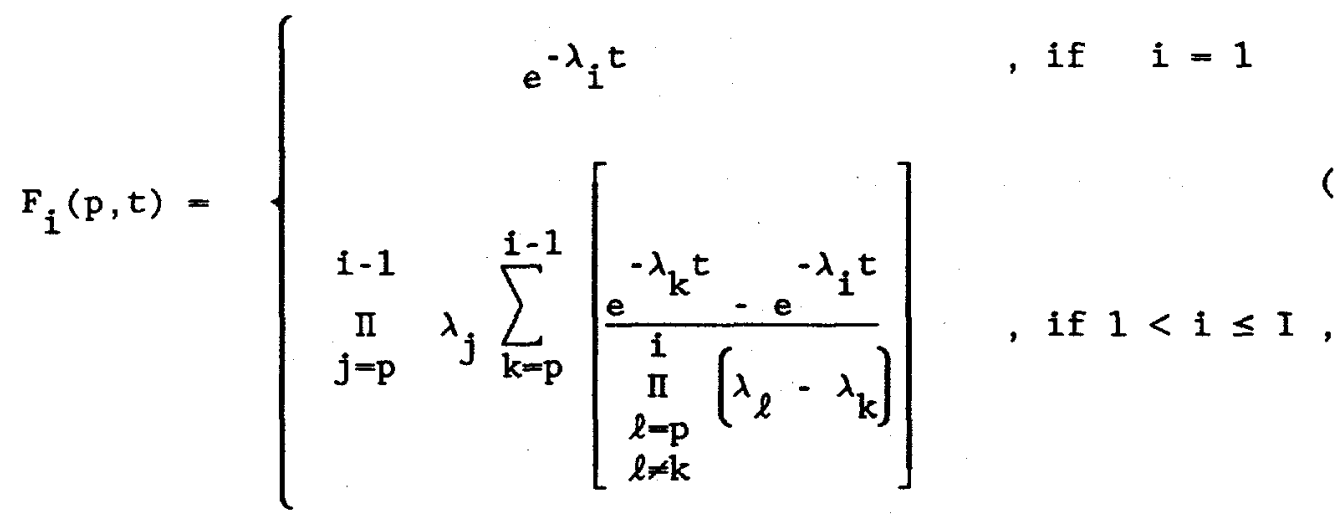

where $F_{1}(p, t)$ is the fraction of parent $p$ decaying to species $i$ over time $t$.

The fractions generated by Equation 2.29 can be used to find the emplaced inventory of species $i$ at $t$ by substituting these fractions into

$$
N_{i}(t)=\sum_{p=1}^{i} F(p, t) N_{p}(0),
$$

where $N_{p}(0)$ is the initial inventory of species $p$ (atoms/mass of waste matrix).

Equation 2.30 is applied if the user specifies a release time that is greater than zero. This option is useful if one assumes that the waste containers or an engineered barrier in the near-field would prohibit farfield transport for a specified period of time.

The governing equation for the rate at which atoms of species $i$ leach from the waste matrix is given by 


$$
R_{i}(t)=N_{i}(t) \frac{d M(t)}{d t},
$$

where $M$ is the (intact) mass of waste matrix material at time $t$. If the user selects the constant-leach-rate option, then

$$
\frac{d M(t)}{d t}=-\frac{M_{0}}{T_{L}} \text {, }
$$

where $1 / T_{L}$ is the leach rate (time ${ }^{-1}$ ) and $M_{0}$ is the initial waste mass $(m)$.

The solution to Equation 2.32 is

$$
M(t)=M_{0}\left(1-t / T_{L}\right)
$$

For the exponential-leach-rate option,

$$
\frac{d M(t)}{d t}=-\frac{M(t)}{T_{L}}
$$

Solving Equation 2.34 results in

$$
M(t)=M_{0} e^{-t / T_{L}}
$$

The "leached but undissolved" inventory is described by the following equations

$$
\frac{d U_{i}}{d t}= \begin{cases}-\lambda_{i} U_{i}+R_{i}-s_{i} & , \text { if } i=1 \\ -\lambda_{i} U_{i}+\lambda_{i-1} U_{i-1}+R_{i}-s_{i} & , \text { if } 1<i \leq I\end{cases}
$$

where $U_{1}$ is the leached-but-undissolved inventory of species $i$ (atoms), $S_{i}$ the rate at which atoms in the leached-but-undissolved inventory enter solution, and $R_{i}$ is the leach rate given by Equation 2.31 .

The formulation for $S_{i}$ depends on the source flow model chosen, flow through or mixing cell, and whether solubilities are treated. Thus, 
rather than formulating $S_{i}$ in this discussion (which NEFTRAN II does not explicitly use) determination of radionuclide concentrations for the two source flow models is discussed in the implementation section (Section 2.3.2). This section is concluded with the theory for the mixing-cell model.

Figure 2.3 illustrates the basic idea of the mixing-cell source model. Water at a certain volumetric flow rate, $Q_{s}$ (volume time), moves through a constant volume, $V_{f}$, and dilutes any dissolved material in the volume. The rate at which dissolved material leaves the mixing cell is

$$
S_{c}(t)=C(t) Q_{s}(t)
$$

where $S_{C}(t)$ is the source rate (in mass per unit time) and $C(t)$ the solute concentration (in mass/volume).

The operation of the mixing cell can be illustrated with a simple example. Given a cell of constant pore volume, $V_{f}$, with a given flow rate, $Q_{s}$, and initial concentration of a dissolved species, $C_{0}$, a mass balance shows that the concentration of the species leaving the cell is described by

$$
\frac{d C}{d t}=-C\left(\frac{Q_{s}}{V_{f}}\right)
$$

Integration using the initial condition $C(t=0)=C_{0}$ gives

$$
C(t)=C_{o} e^{\left(\frac{Q_{s} t}{V_{f}}\right)}
$$

Substituting Equation 2.39 into Equation 2.37 gives

$$
s_{c}(t)=c_{o} Q_{s} e^{\left(\frac{Q_{s} t}{V_{f}}\right)}
$$

If the user opts, NEFTRAN II will determine whether a mixing-cell-source model is appropriate. The basis for choosing a mixing-cell model is the average particle residence time, $T_{r}$, given by

$$
T_{r}=\frac{V_{f}}{Q_{s}} .
$$




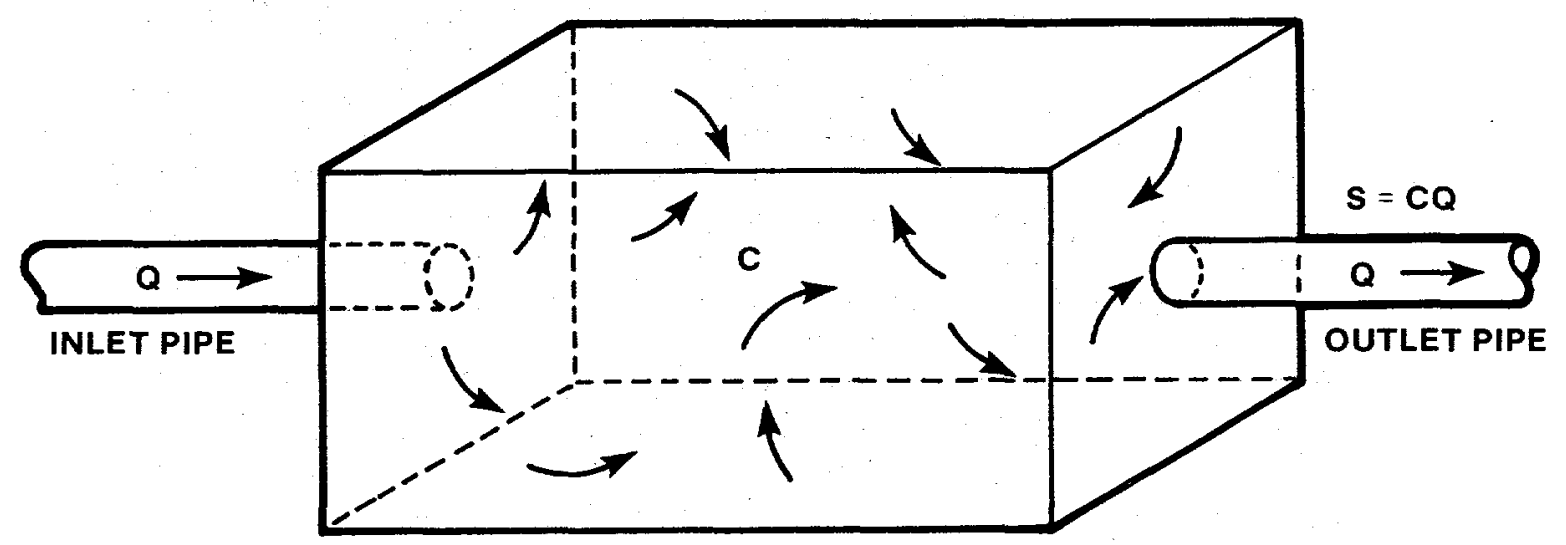

Figure 2.3. The Mixing Cell 
If $T_{r}$ is small compared to the average migration time of an isotope, then it is assumed that all of the material leaves the source volume rapidly, and it is not necessary to use a mixing-cell-source model. Alternatively, if $T_{r}$ is large compared to the average migration time, there is the possibility of dilution of the source concentration and a mixing cell source is appropriate.

\subsubsection{Implementation}

The various source model options are all implemented over discrete time steps, $\Delta t_{s}$. The user can specify $\Delta t_{s}$ or allow NEFTRAN II to determine an appropriate value. If the user chooses to specify $\Delta t_{s}$, it is strongly recommended that the guidelines implemented in NEFTRAN II to determine $\Delta t_{s}$ be followed.

Source Model Time Step Determination. There are four basic criteria used to determine a time step for the source model, these are:

1. ensure that the leach pulse is adequately represented,

2. ensure that radioactive decay is accounted for accurately,

3. ensure that the time step is less than the average residence time in the mixing cell, and

4. for the case of time-dependent flow rates through the repository, ensure that the time step is small enough to capture the peak time-dependent flow rate.

The impact of each criterion on $\Delta t_{s}$ depends on the source options chosen and the properties of the source region and decay chains. The criteria are defined further by a set of increment-determination parameters. These parameters have default values in the code or can be input directly by the user (see section 4.3.5). The description that follows is based on the default values.

LEACH PULSE - If leaching is being treated as part of the source model, then at least five time steps across the leach pulse are required.

DECAY - The time step cannot exceed 0.5 times the smallest "important" half-life for all important isotopes. An important isotope is defined as an isotope that has a mean trave1 time less than the problem simulation time, and a half life and mean velocity such that a significant fraction of its initial inventory will survive decay to discharge. Therefore, an isotope is important if its initial inventory will discharge during the problem time and if the isotope travel time is less than twenty half lives.

RESIDENCE TIME - The user has the option to use a mixing-cell source, a flow-through source or to let the code decide which type of source model is appropriate (see Section 4.3.1). If the mixing cell source is being implemented, then $\Delta t_{s}$ cannot exceed 0.2 times the mixing cell residence time, $T_{r}$. 
FLOW PEAK - For time-dependent flowrates through the repository, $\Delta t_{\text {, }}$ should capture the peak time-dependent flow rate. This is done by mult1plying a fraction, 0.8 , by the peak flow rate. The times greater than and less than the peak flow rate at which this fractional flow rate occurs are determined. These times dictate the time range that bounds $\Delta t$, to ensure that the peak flow rate is captured. This algorithm may not be sufficient for all time-dependent flow rate profiles.

Leach-Limited Source. If the leach-limited only source option is selected (i.e., solubility limits are not applied), the rate at which a particular radionuclide enters solution, $R_{i}$, is given by Equation 2.31 . $\mathrm{N}_{\mathrm{i}}$ is given by Equations 2.30 and 2.29 and $\mathrm{dM} / \mathrm{dt}$ is found from either Equation 2.32 or Equation 2.34. If the flow-through source option is selected then $R_{i}$ is the only information that is needed to determine the source rates for each isotope. In practice, the total amount leached from $t$ to $t+\Delta t_{s}$ is found and decay is accounted for by finding the average amount available during this time interval.

If the mixing-cell option is selected, the source concentration for species $i$ must be determined to find the source rate for species $i$ and is given by

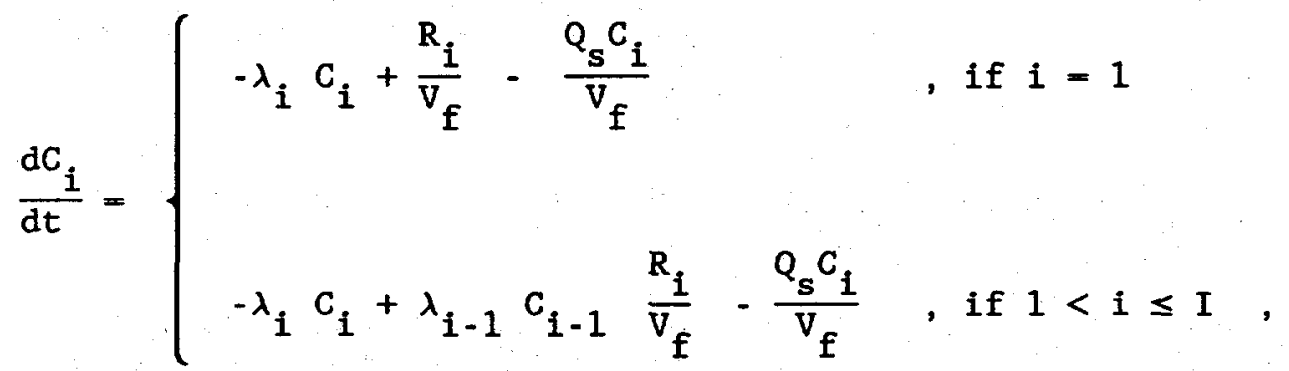

where $C_{i}$ is the concentration of species i (atoms/volume ${ }^{3}$ ).

Equation 2.37 is used to determine the source rates for each isotope, again as averages, from to $t+\Delta t_{s}$.

Solubility-Limited Source. If the solubility-limited option is selected (i.e., leach-1imits are not applied), NEFTRAN II places the entire radionuclide inventory in the leached-but-undissolved inventory at the time of release. Then, for the flow-through-source option, the source concentration $\left(C_{1}\right)$ is given by the minimum of $U_{1}(t) /\left(Q_{s} \Delta t_{s}\right)$ or $L_{i}(t)$, where $L_{i}(t)$ is the solubility of isotope $I$ (atoms/volume of water).

$C_{1}$ is the minimum of the amount that can dissolve without exceeding the solubility limit or the inventory that is left. In other words, as long as there is more mass than can be dissolved, the source rate will be based on the solubility limit of the species. Once the mass is depleted enough to be less than the mass that can dissolve, the source rate will be based on the amount of mass that is left. 
If the mixing-cell source option is selected, an intermediate source concentration $(\tilde{C})$ is found by accounting for decay, production, and dilution; that is,

$$
\frac{d \widetilde{\mathrm{C}}_{i}}{\mathrm{dt}}=\left\{\begin{array}{lll}
-\lambda_{i} \tilde{\mathrm{c}}_{i}-\gamma \widetilde{\mathrm{C}}_{i} & \text {, if } 1=1 \\
-\lambda_{i} \tilde{\mathrm{C}}_{i}+\lambda_{i-1} & \tilde{\mathrm{c}}_{i-1}-\gamma \tilde{\mathrm{C}}_{i} & \text {, if } 1<i \leq 1
\end{array},\right.
$$

where $\gamma\left(=Q_{s} / N_{f}=1 / T_{r}\right)$ is the dilution factor.

Solubilities are accounted for by using the equation

$$
c_{i}(t)=\operatorname{MIN}\left[\tilde{c}_{i}(t)+\frac{U_{i}(t)}{V}, L_{i}(t)\right] .
$$

Combined Leach and Solubility Limits. The user can elect to apply a leach rate to the waste matrix and solubility limits to radionuclides in solution. In this case, the rate at which radionuclides escape the waste matrix and enter the leached-but-undissolved inventory is given by Equations 2.31 and 2.32 or 2.34 , depending on whether a constant leach or an exponential leach rate is being used. The leached-but-undissolved inventory is then found from Equation 2.36.

If the flow-through source option has been selected, the source concentration is given as discussed above for a solubility-limited source. Otherwise, for the mixing-cell source option, the source is determined in two steps, from Equation 2.43 and Equation 2.44 .

Apportioning Solubility Limits. In the equations presented above, the solubility limit for species $i, L_{1}(t)$, was assumed to be time-dependent. However, in practice, solubilities are only available for elements and are constant with time, provided solvent properties are constant. In a typical NEFTRAN II application there may be several isotopes of the same element competing for dissolution. The approach taken in NEFTRAN II is to partition the amount of a particular element that can be dissolved into amounts proportional to the individual isotopes. These isotopes are undergoing radioactive decay/production as time evolves and therefore, their proportions and hence their solubility limits, become timedependent. 
To illustrate the proportioning, suppose element $E$ has isotopes $E_{1}, E_{2}$, $\ldots, E_{k}$. The solubility limit for species $E_{1}$ is given by

$$
L_{i}(t)=\frac{U_{i}(t)}{\sum_{j=1}^{k} U_{j}(t)} \cdot L_{E}
$$

where $L_{E}$ is the solubility limit for element $E$ (atoms/volume).

Thus, the effective solubility limit for species $i, L_{1}$, is time-dependent if there are two or more isotopes of element $E$ with different decay or production rates.

Time-Dependent Flow Rates Through the Source, As discussed above, to determine source rates based on solubility limits, the flow rate through the source (repository) is used. The user may provide time-dependent flow rates through the source region from an external file (Section 4.3.1). Although flow rates are also used for the mixing-cell source model, the option to use time-dependent flow rates is currently only available in conjunction with the flow-through source model.

Time-dependent flow rates through the source can also occur if the user provides time-dependent fluid velocities from an external file (Section 4.3.1). In this case, the velocity for the first leg in the migration path is used to determine a time-dependent flow rate through the source. If both options are implemented (i.e., external file of time-dependent flow rates through the source, and an external file of time-dependent fluid flow velocities), the time-dependent flow rates through the source are utilized by the source model.

\subsection{Distributed Velocity Method (DVM) Transport Model}

The usual run-mode for the NEFTRAN II code implements the DVM transport option. Convective transport is simulated by moving groups or packets of particles (representing dissolved radionuclides) along the flow field over each time step. Dispersion is simulated by allowing the packets to spread simultaneous with convective transport. Thus, DVM is similar to particle tracking methods or the method-of-characteristic. However, what sets DVM apart from these transport simulation methods is the ability to incorporate radioactive decay chains with the direct simulation technique. The following sections describe the theoretical basis of DVM, as well as the numerical implementation of the DVM transport mode1. The capability unique to NEFTRAN II, DVM transport in time-dependent velocity fields, is also discussed.

\subsubsection{Theory}

The convective-dispersion transport equation in one dimension can be written as 


$$
\frac{\partial \rho}{\partial t}=D \frac{\partial^{2} \rho}{\partial x^{2}}=\bar{v} \frac{\partial \rho}{\partial x}+s
$$

where $\rho$ is the particle density (atoms/volume), $\bar{v}$ is the average particle velocity (length/time) and $D$ is the dispersion coefficient (length ${ }^{2} /$ time). These units of $\rho$ are used because they facilitate the simulation of radioactive decay and can be easily converted to the traditional unit of concentration.

Assuming negligible molecular diffusion, the dispersion coefficient (D) is defined as

$$
D=\alpha|\bar{v}|
$$

where $\alpha$ is the dispersivity (length). In ground-water systems, molecular diffusion is negligible when transport by convection dominates molecular diffusion or, if it does not dominate, is the most significant pathway. By defining the dispersion coefficient in this manner, NEFTRAN II simulates mechanical dispersion and not hydrodynamic dispersion which includes the effects of molecular diffusion.

When $\rho=0$ at the infinite boundaries, the solution to Equation 2.46 contains two terms: a particular solution and a complementary solution. Taking the density of particles at time $t^{\prime}$ to be $\rho\left(x^{\prime}, t^{\prime}\right)$, the complementary solution is

$$
\rho_{0}(x, t)=\int_{-\infty}^{\infty} d x^{\prime} \rho\left(x^{\prime}, t^{\prime}\right) G\left(x-x^{\prime}, t-t^{\prime}\right)
$$

the particular solution of Equation 2.46 yields the complete solution

$$
\rho(x, t)=\rho_{0}(x, t)+\int_{t^{\prime}}^{t} d \tau \int_{x_{0}}^{x_{1}} d x^{\prime} S\left(x^{\prime}, \tau\right) G\left(x-x^{\prime}, t-\tau\right)
$$

The Green's function, $G\left(x-x^{\prime}, t-t^{\prime}\right)$, in Equations 2.48 and 2.49 may be written as

$$
G\left(x-x^{\prime}, t-t^{\prime}\right)=\frac{1}{\sqrt{2 \pi} \sigma_{x}} \exp \left\{-\frac{\left[\left(x-x^{\prime}\right)-\bar{v}\left(t-t^{\prime}\right)\right]^{2}}{2 \sigma_{x}^{2}}\right\},
$$


where the variance is

$$
\sigma_{x}^{2}=2 D\left(t \cdot t^{\prime}\right)
$$

$G\left(x-x^{\prime}, t-t^{\prime}\right)$ is a spatial Green's function, as indicated by the units of $\sigma_{x}$ (i.e., length) and $G$ (i.e., length ${ }^{-1}$ ).

Equation 2.50 may be recast as a velocity distribution by considering the illustration in Figure 2.4. This figure shows the evolution of the Green's function over ( $\left.t-t^{\prime}\right)$ from an instantaneous point source function to a Gaussian distribution. Particles arriving at the mean position of this spatial distribution have travelled from $x^{\prime}$ with velocity $\bar{v}$. Particles at position $x$, however, have travelled from $x$ ' with an average velocity

$$
v=\frac{\left(x-x^{\prime}\right)}{\left(t-t^{\prime}\right)}
$$

If Equation 2.52 is substituted into Equations 2.50 and 2.51 then

$$
G\left(x-x^{\prime}, t-t^{\prime}\right)=\frac{H\left(v, t-t^{\prime}\right)}{\left(t-t^{\prime}\right)}
$$

where

$$
H\left(v, t-t^{\prime}\right)=\frac{1}{\sqrt{2 \pi} \sigma_{v}} \exp \left[-\frac{(v-\bar{v})^{2}}{2 \sigma_{v}^{2}}\right] \text {, }
$$

and

$$
\sigma_{v}=\sqrt{\frac{2 D}{\left(t-t^{\prime}\right)}}=\frac{\sigma_{x}}{\left(t-t^{\prime}\right)}
$$

Quantity $\mathrm{H}$ is a Green's function in velocity space as indicated by the units of $\sigma_{v}$ (i.e., length/time) and $H$ (i.e., time/length).

Substituting Equation 2.53 into Equation 2.49, gives

$$
\rho(x, t)=\rho_{0}(x, t)+\int_{t^{\prime}}^{t} d \tau \int_{x_{0}}^{x_{1}} S\left(x^{\prime}, \tau\right) H(v, t-\tau) /(t-\tau) d x^{\prime}
$$




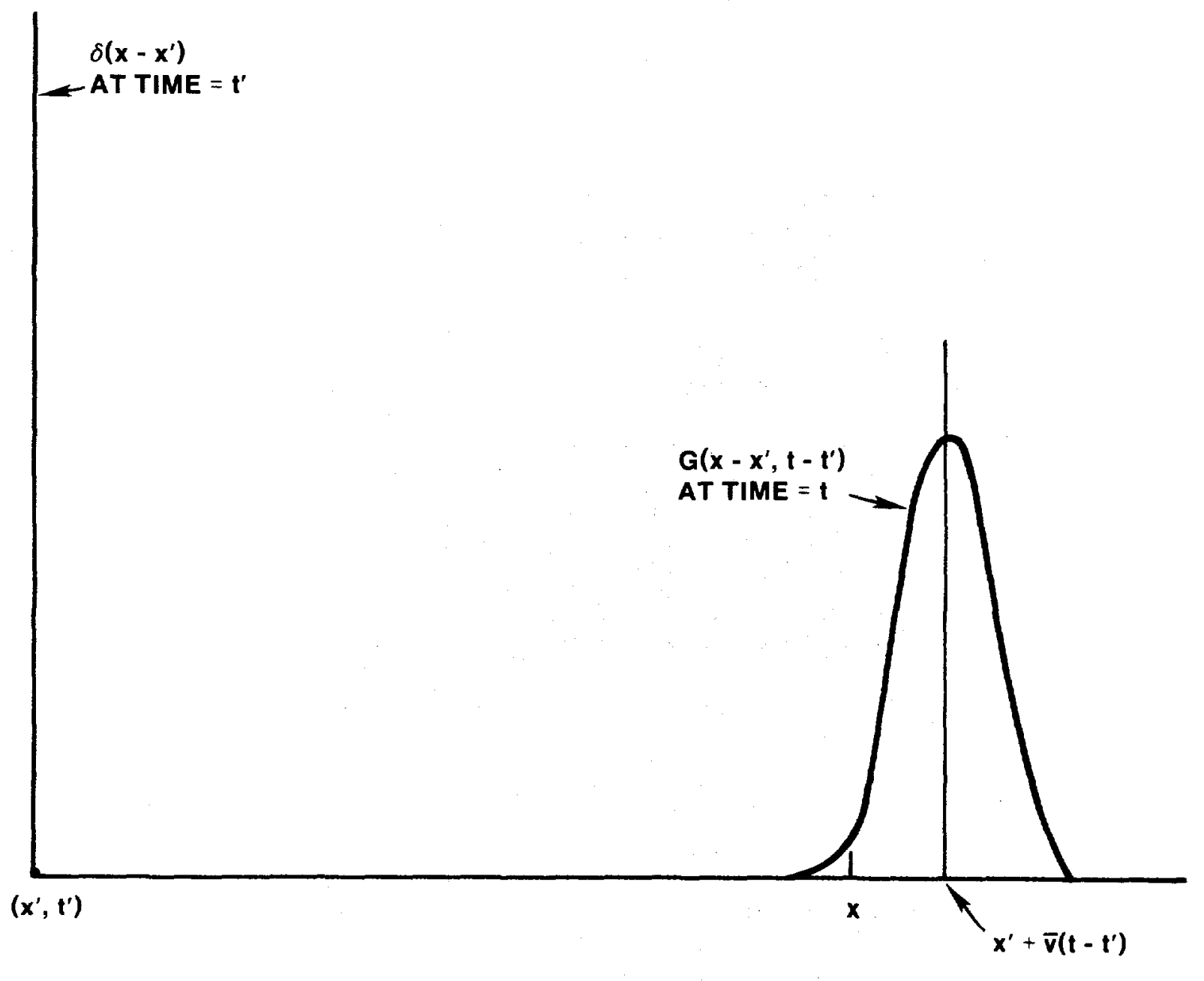

Figure 2.4. The Spatial Green's Function 
Recalling Equation 2.52, Equation 2.56 can be written as

$$
\rho(x, t)=\rho_{0}(x, t)+\int_{t^{\prime}}^{t} d \tau \int_{v_{0}}^{v_{1}} S\left(x^{\prime}, \tau\right) H(v, t-\tau) d v
$$

where

$$
v_{0}=\frac{x-x_{0}}{t-t^{\prime}}
$$

and

$$
v_{1}=\frac{x-x_{1}}{t-t^{\prime}}
$$

Thus the transport of an ensemble of particles at position $x^{\prime}$ and time $t^{\prime}$ to position $x$ at a later time $t$ can be accomplished by summing over a suitable velocity range weighted by the Green's function in velocity space.

The theoretical basis for DVM is the process of mechanical dispersion which occurs with solute transport through porous media. Specifically, the physical concept embodied in Equation 2.57 is due to heterogeneity of the flow field, a number of alternate paths exist for particles to migrate from $x^{\prime}$ to $x$. The underlying assumption is that these paths may be characterized by a continuum of migration times and average velocity components in the direction of flow. By distributing these average velocities according to the Gaussian function developed earlier (Equation 2.54), DVM has been specialized to the conventional Fickian treatment of dispersion. Although there has been some criticism of this conventional Fickian treatment for modeling mechanical dispersion, currently, an alternative does not exist; however, in principle, the DVM technique could be applied to any specified velocity distribution.

\subsubsection{Implementation}

This section briefly describes the manner in which DVM is numerically implemented. The discussion begins with a single decaying radionuclide. The analysis is then extended to the treatment of a decay chain.

To motivate this discussion of numerical implementation, recal1 Figure 2.4 which illustrates the movement of an instantaneous point source at position $x^{\prime}$ and time $t^{\prime}$ to a Gaussian profile at time $t>t^{\prime}$ and position $x$ given by $x=x^{\prime}+v\left(t-t^{\prime}\right)$.

In the numerical implementation of DVM, this can be visualized as shown in Figure 2.5. Here, the spatial dimension $x$ is discretized into grid blocks of equal length. The "point source" is confined to a single grid block as shown. However, to model dispersive spreading of the material as it is transported from $t^{\prime}$ to $t$, particles in the source block are given a distribution of velocities. As illustrated, particles assigned 


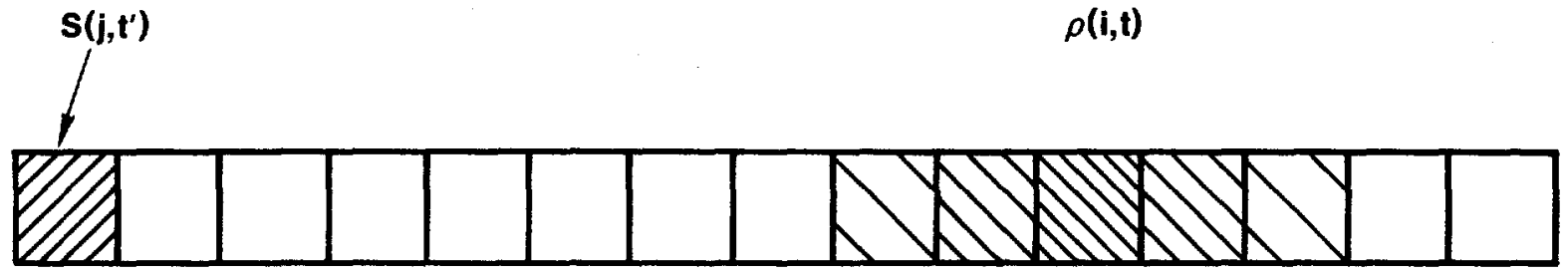
$j \mathrm{j}+1$

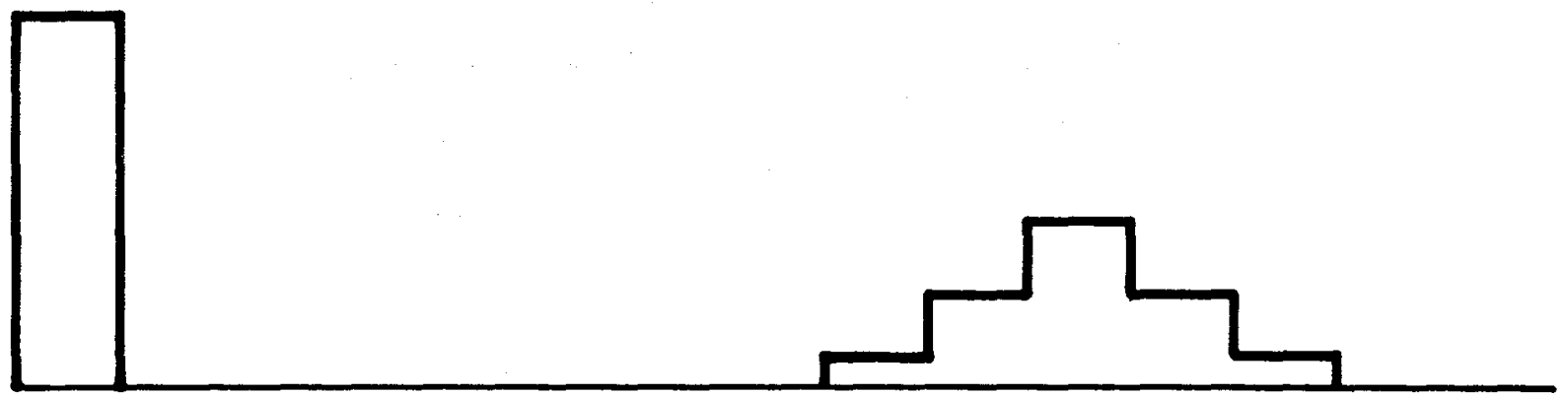

Figure 2.5. Numerical Simulation of the Transport of an Instantaneous Point Source 
to the higher velocity intervals travel farther than those assigned to the lower velocity intervals. If the velocity intervals are determined from a Gaussian distribution (see Equations 2.54 and 2.55), then the particle density $\rho(x, t)$ approximates a Gaussian distribution in the spatial dimension.

So, for the $\ell$ th leg having mean fluid velocity, $\bar{v}_{f l}$ (length/time) and dispersivity $\alpha_{\ell}$ (length), velocities for the $j$ th velocity interval, $v(l, r, j)$, are found for species $r$ as follows:

$$
v(\ell, r, j)=\varepsilon_{j} \sqrt{\frac{2 \alpha_{\ell} \bar{v}_{f \ell}}{\Delta t R_{\ell}}+\frac{\bar{v}_{f \ell}}{R_{\ell}}},
$$

where $R_{\ell}$ is the retardation factor (dimensionless) for species $r$ in the $\ell$ th ${ }^{l e g}$, and $\varepsilon_{j}$ is the $j$ th abcissa from a standard normal distribution given by

$$
\frac{2 j-1}{2 N_{v}}=\int_{-\infty}^{\varepsilon} j \frac{1}{\sqrt{2 \pi}} e^{-0.5 u^{2}} d u, j=1, \ldots, N_{v} \text {, }
$$

where $\mathrm{N}_{\mathrm{v}}$ is the number of velocity intervals.

For clarity, Figure 2.5 presents the DVM transport, as applied to a single source block at time $t^{\prime}$ and the several resulting receiver blocks at time $t>t^{\prime}$. In actual application, however, it is numerically more efficient to determine the contributions to a single receiver block at time $t$ from multiple source blocks at time $t^{\prime}<t$. This approach is discussed in the following sections.

One Decaying Radionuclide. Here, only the propagation of the density function $\rho\left(x^{\prime}, t^{\prime}\right)$ from time $t^{\prime}$ to time $t$ (Equation 2.57) is considered. There are $\mathrm{N}_{\mathrm{x}}$ equal-space increments of length $\Delta \mathrm{x}$ and the time increment $\Delta t$ is taken to be constant. The velocity dimension is divided into $\mathrm{N}_{\mathrm{v}}$ increments based on equal probability. While DVM can be generalized to variable spatial and time increments, such generalization is not considered in NEFTRAN II as will become clear in the discussion that follows.

Propagation of densities over $\Delta t$ for velocity subgroup $j$ may be written as 


$$
\begin{aligned}
\Delta \rho(i, j, t) & =D_{f} W(j)\left\{M_{f}(j) \rho\left(i-k_{j}, t^{\prime}\right\}\right. \\
& \left.+\left[1-M_{f}(j)\right] \rho\left(i-k_{j}-1, t^{\prime}\right)\right\}
\end{aligned}
$$

Although the argument 1ist in Equation 2.61 appears formidable, it can be readily understood. As indicated there, the contribution to receiver block $i$ for velocity interval $j$ is determined by three fractions: $a$ geometric mixing fraction, $M_{f}$, a velocity-interval fraction, $W$, and a decay fraction, $D_{f}$.

$M_{f}$ may be understood by referring to Figure 2.6. Looking at velocity interval $j$, one sees that there are, in general, two contributions to receiver block $i$. One is a packet of particles coming from donor block $i-k$ and the other is a packet of particles coming from donor block $i-k-1$. Although not intuitively obvious, the reason there are only two contributions is because the physical dimension of a packet of particles is the actual grid block size. As is also indicated in Figure 2.5, there is generally only partial overlap of the propagated block contents with receiver block $i$. The donor block index $k_{j}$ is

$$
k_{j}=\left[\left[\frac{v_{j} \Delta t}{\Delta x}\right]\right]
$$

where $[[z]]$ is the greatest integer $\leq z$,

and $v_{j}$ is determined from

$$
\left(\frac{j-\frac{1}{2}}{N_{v}}\right)=\frac{1}{\sqrt{2 \pi} \sigma_{v}} \int_{-\infty}^{v_{j}} \exp \left(-\frac{(v-\bar{v})^{2}}{2 \sigma_{v}^{2}}\right) d v
$$

The corresponding mixing fraction is

$$
M_{f}(j)=1-\left(\frac{v_{j} \Delta t}{\Delta x}-k_{j}\right) \text {. }
$$




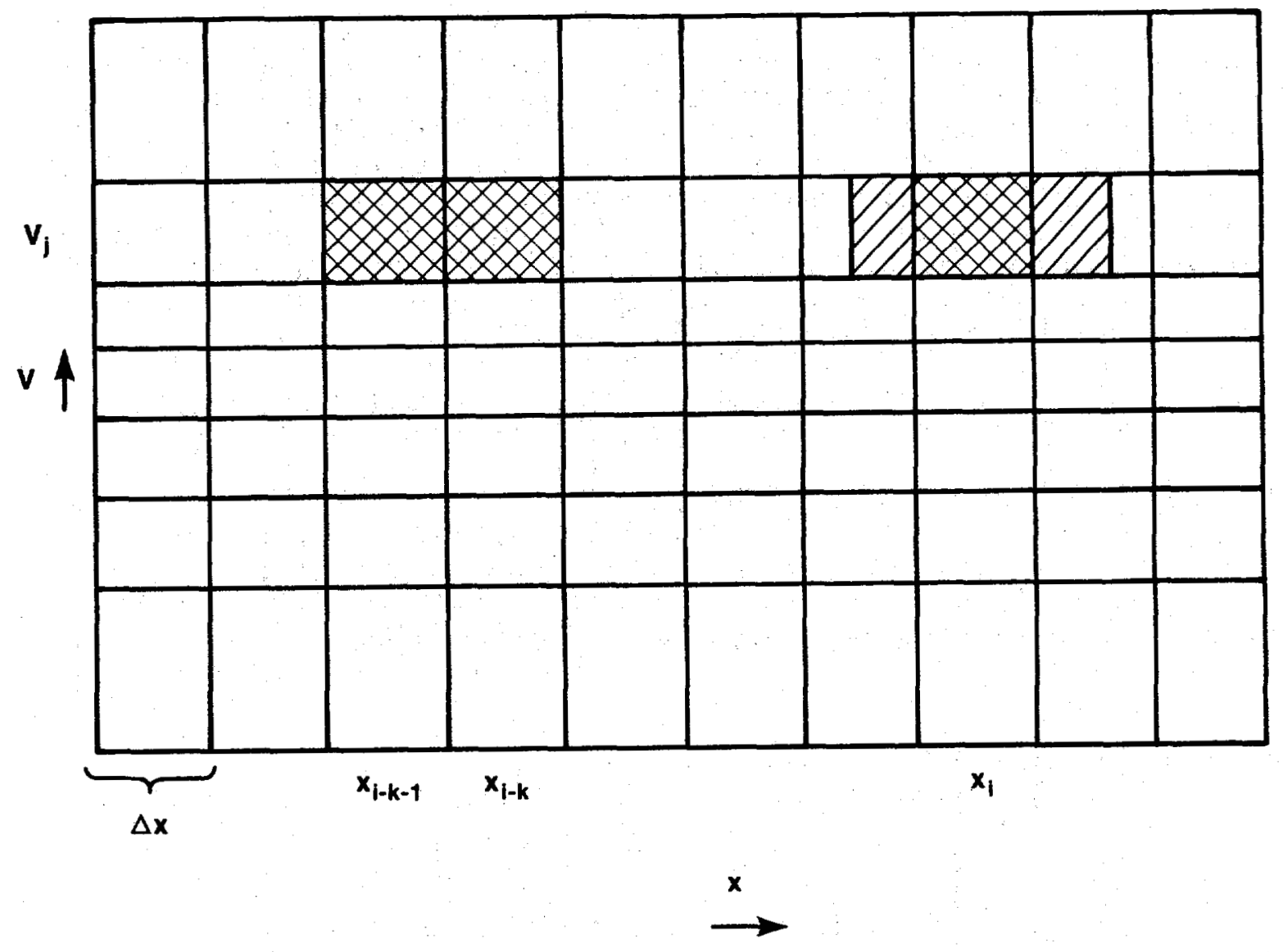

Figure 2.6. Illustration of the Mixing Fraction Concept 
Because the velocity dimension is divided into intervals assuming equal probability, the weight $W(j)$ assigned the $j^{\text {th }}$ velocity interval is

$$
W(j)=\frac{1}{N_{v}}
$$

The decay fraction is taken to be

$$
D_{f}=e^{-\lambda \Delta t}
$$

With these three fractions defined, Equation 2.61 is summed over all velocity intervals to obtain the total particle density in grid block 1 :

$$
\begin{array}{r}
\rho(i, t)=\frac{D_{f}}{N_{v}} \sum_{j=1}^{N_{v}}\left\{M_{f}(j) \rho\left[i-k_{j}, t^{\prime}\right)+\left[1-M_{f}(j)\right\} .\right. \\
\\
\left.\rho\left(i-k_{j}-1, t^{\prime}\right)\right\} .
\end{array}
$$

As a last step in this discussion, Equation 2.67 is rewritten in a more computationally efficient form. To do this, the possibility of degeneracy with respect to the index $k_{j}$ is addressed. This degeneracy could mean either

$$
k_{j+1}=k_{j} \quad \text { or } \quad k_{j+1}=k_{j}+1
$$

Taking such degeneracies into account yields the expression

$$
\rho(i, t)=\sum_{j=1}^{N_{B}(i)} B(j) \rho\left(i-k_{j}, t^{\prime}\right)
$$

Quantities $N_{B}(i)$ and $B(j)$ are most easily obtained by a computational procedure which makes the tests of Equation 2.68 and accumulates the coefficients to form the matrix of $B$.

It is important to note that, as long as $\Delta x$ and $\Delta t$ are constants, $B$ is a simple vector which contains at most $2 \cdot \mathrm{N}_{\mathrm{v}}$ terms. Furthermore, $\mathrm{B}(\mathrm{j})$ may be computed during the initial setup before the actual transport simulation is conducted. For unequal grid block increments $\Delta x, B$ would be 
dependent on grid block index $i$, and for unequal time increments $\Delta t, B$ would have to be re-evaluated for each change in time increment. Thus, it is most efficient to maintain fixed $\Delta x$ and $\Delta t$.

The implementation just described corresponds to DVM as it was implemented into the NWFT/DVM code (Campbe11, and others, 1981a, 1981b). The same implementation exists in NEFTRAN (Longsine, and others, 1987) and in NEFTRAN II. However, in the latter two codes, the restrictions of constant $\Delta x$ and $\Delta t$ were relaxed. In NWFT/DVM, the legs along the migration path were combined into a single leg with each isotope assigned an average velocity over that leg which preserved total mean migration time. This leg-averaged path option is still in NEFTRAN and NEFTRAN II, however, with the addition of the matrix diffusion capability to NEFTRAN, it became necessary to treat each leg individually. This treatment was necessary since the residence time in a leg characterized by dual porosity is one of the primary factors that determines the amount of diffusion. The implementation in NEFTRAN and NEFTRAN II is to treat each leg as a distinct unit with (1) a fixed space step for the leg and transport of particles in the interior of the leg described by the $B$ array above, (2) allowing the space step to be different for each leg along the migration path, and (3) computing the fractions of grid blocks in one leg that contribute to blocks in another leg over a time step as a separate model. Thus, a new model, referred to as the leg-to-leg transfer model, was implemented as described in Section 2.4.9.

NEFTRAN II relaxes the fixed time-step restriction. NEFTRAN II simulates time-dependent flow fields by approximating the changing velocities with a step function (see Section 2.4.11). In particular, the user defines time intervals over which the fluid velocity is relatively constant and sequences these intervals from release time to the end of simulation. over each of these time intervals, a time step is chosen and implementation occurs as described above. However, because the intervals can be of different length and with the changing fluid velocities, it is likely that accuracy and efficiency considerations will dictate the use of a different time step for each time interval. Thus, following each time interval, the code recomputes the transport fractions based on the new velocities and new time steps to be used for the next time interval.

A Chain of Radionuclides. Here the starting point is similar to Equation 2.61 , and for species $r$ of a radioactive decay chain it may be written as follows

$$
\begin{aligned}
\Delta \rho(i, j, r, r-p, t)= & D_{f}(r, r-p) W(j) \bullet \\
& \left\{M_{f}(j, r, r-p) \rho\left(i-k_{j}, r-p, t^{\prime}\right)\right. \\
& +\left[1-M_{f}(j, r, r-p)\right] \\
& \left.\rho\left(i-k_{j}-1, r-p, t^{\prime}\right)\right\}
\end{aligned}
$$


In this equation $\Delta \rho$ is the incremental particle density for isotope $r$, grid block $i$, velocity subgroup $j$, and time $t$ which arises from decay of isotope $r$ - $p$ over time interval $t-t^{\prime}$. If $p=0$, Equation 2.70 simplifies to Equation 2.61 .

$M_{f}$, the mixing fraction, is determined by Equations 2.62 and 2.64 as before. The only difference is the velocity. Because of sorption effects, the velocity will, in general, change with species during the decay $r-p \rightarrow r-p+1 \ldots \rightarrow r$ which occurs during time step $\Delta t$. Hence, in this case, the velocity $v(j, r, r-p)$ represents a species average over the time interval $\Delta t=t-t^{\prime}$. The model used in NEFTRAN II to determine these species average velocities is presented in Section 2.4.6. $W$, the velocity subgroup fraction, is taken from Equation 2.65 as before.

The role of quantity $D_{f}$ in Equation 2.70 is expanded beyond that in Equation 2.61. In Equation 2.61, $D_{f}$ denotes that fraction which survives decay during $\Delta t$. In Equation $2.70, D_{f}$ pertains to decay only when $p=0$, i.e.,

$$
D_{f}(r, r-p)=e^{-\lambda \Delta t}, \quad p=0 .
$$

Otherwise, this quantity denotes production of species $r$ from species $r$ - $p$, and may be developed from the Bateman equations (see Section 2.3 .1 ) as

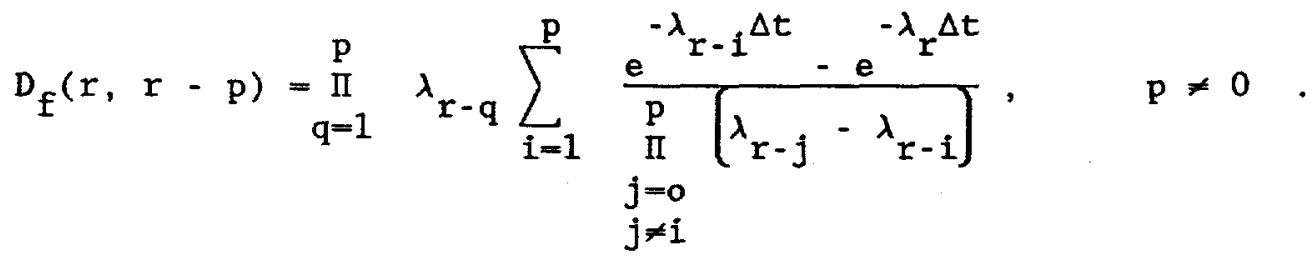

This equation, when coupled with appropriate mixing and weighting functions, may be viewed as a model for evaluating that portion of the source integral in Equation 2.57 which is appropriate for radioactive decay processes.

To complete the analysis of radioactive decay chains, Equation 2.70 is summed over all velocity subgroups $j$ and all parents $p$ of species $r$. This yields

$$
\begin{aligned}
\rho(i, r, t)= & \sum_{p=0}^{N_{p}(r)} \sum_{j=1}^{N_{v}} D_{f}(r, r-p) W(j)\left\{M_{f}(j, r, r-p) \bullet\right. \\
& \rho\left[i-k_{j}, r-p, t^{\prime}\right)+\left[1-M_{f}(j, r, r-p)\right] \bullet \\
& \left.\rho\left(i-k_{j}-1, r-p, t^{\prime}\right)\right\} .
\end{aligned}
$$


Recollecting the sum then gives the working equation

$$
\begin{aligned}
\rho(i, r, t)= & \sum_{p=0}^{N_{p}(r)} \sum_{j=1}^{N_{B}(i, r, r-p)} B(j, r, r-p) \\
& \rho\left(i-k_{j}, r-p, t^{\prime}\right) .
\end{aligned}
$$

The upper bounds in Equation 2.74 are expressed as functions. The dependence $N_{p}(r)$ reflects the fact that, in general, each radionuclide has a different number of parents. Furthermore, one may want to arbitrarily impose a bound on the parent sum. The limit $\mathrm{N}_{B}(i, r, r-p)$ gives the number of grid blocks which contribute to a receiver block $i$. The limit carries radionuclide indices because of the transport velocity model (Section 2.4.6). As in the case of a single decaying nuclide, $B$ will not vary with grid block or time step indices provided that $\Delta x$ and $\Delta t$ do not vary. Thus the $B$ matrix may be generated during the setup process. For each $r$, the $B$ matrix can be viewed as $N_{p}(r)+1$ separate vectors each having dimension less than or equal to $2 \cdot \mathrm{N}_{\mathrm{v}}$. Hence, computer storage is not a problem. For purposes of comparison with other numerical techniques, Equation 2.74 is an explicit relation in that each term on the right-hand side is known prior to solution for the unknown density $\rho(i, r, t)$.

The implementation of DVM over a given time interval requires a fixed space step for each leg and a fixed time step for the time interval. The user can specify the space and/or time steps or allow NEFTRAN II to determine appropriate values. If the user chooses to specify the space and/or time steps, it is strongly recommended that the guidelines implemented in NEFTRAN II to determine $\Delta x$ and $\Delta t$ be followed. For this reason, the next two sections discuss the guidelines in detail. The discussion follows the code logic which first finds the time step and then the space $\operatorname{step}(s)$.

\subsubsection{Time Step Determination}

The three basic criteria used to determine a time step for transport are:

1. ensure that packets cannot jump from source to discharge (for the path-averaged simulations) or skip a leg (for leg-to-leg simulations) in a single time step,

2. ensure that there are adequate points to resolve the breakthrough portion of the discharge curves (for path-averaged simulations only), and

3. require a minimum nominal number of time steps for the simulation. 
The extent to which each of the criteria restricts the size of $\Delta t$ depends on the migration time, dispersivity, and half-lives modeled. The criteria are defined further by a set of increment-determination parameters. These parameters have default values in the code or can be input by the user (see Section 4.3.5). The description that follows is based on the default values that are appropriate for the repeated runs involved in statistical Monte Carlo simulations.

Avoid Leg Jump. The time step $\Delta t$ is initialized to the minimum travel time which is defined as:

$$
T_{\min }=\frac{L_{p}}{v_{\max }}
$$

for the path-averaged case or

$$
T_{\min }=\operatorname{MIN}\left(\frac{z_{i}}{v_{\max }^{i}}\right)
$$

for the leg-to-leg transfer case. $I_{p}$ is the total migration path length and $Z_{i}$ is the length of leg $i$. For the leg-to-leg transfer case, the minimum is taken over all legs $i$ and for both definitions, $v_{\max }$ (length/ time), is the maximum distributed isotope velocity in the path or leg.

$v_{\max }$ is found based on the normal distribution of isotope velocities (see Section 2.4.2). This is done by approximating the normal distribution of velocities to find the velocity of the last and therefore, largest velocity interval.

Since $v_{\max }$ depends on $\Delta t$ (more precisely, the inverse square root of $\Delta t$ ), the relations

$$
v_{\max } \Delta t \leq L_{p}
$$

and

$$
v_{\max } \Delta t \leq z_{i}
$$

can be transformed into quadratic relations in $\Delta t$. These quadratic forms are solved in NEFTRAN II to get an initial value for $\Delta t$.

Curve Resolution. The curve-resolution restrictions for $\Delta t$ are implemented only for the path-averaged case. This criterion is based on finding the standard deviation in time of the breakthrough curve and setting $\Delta t$ equal to one standard deviation. This ensures that rates are output on the breakthrough portion of the discharge curve. This criterion is only applied for important isotopes. The definition of a important 
isotope is similar to the one discussed in conjunction with the source time step in Section 2.3.2. However, for transport, an isotope is considered important if its average migration time is less than the problem simulation time, and if the average migration time is less than 10 times its half-life, compared to 20 times its half-life for source calculations.

After the minimum (over important isotopes) standard deviation is found, a criterion is applied that does not allow $\mathrm{T}_{\min }$ to be decreased by more than a factor of 0.2 .

Nominal Number of Time Steps. This criterion requires a minimum of 30 time steps for a simulation time, or for the time-dependent velocity case, for a time interval. This criterion is needed since it is possible that the first two criteria resulted in a time step that exceeds the problem simulation time.

\subsubsection{Spatial-Step Determination}

Following the time-step determination, NEFTRAN II finds the spatial gridding. The three criteria used in determining the spatial step are:

1. ensure a nominal number of grid blocks in each leg or the entire transport path,

2. ensure that Courant numbers are bounded from below for important isotopes, and

3. ensure that, for the path-averaged case, the spatial increment, $\Delta x$, is not greater than the length of the source leg.

The extent to which each of the criteria restricts the size of $\Delta x$ depends on the migration time, half-lives of the isotopes being modeled, and the time step. The criteria are defined further by a set of incrementdetermination parameters. These parameters have default values in the code or can be input by the user (see Section 4.3.5). The description that follows is based on the default values for these parameters.

Nominal Number of Blocks. The number of grid blocks for a path-averaged simulation is initialized to 50 meaning $\Delta x$ is initialized to $L p / 50$. Similarly, for a leg-to-leg transfer problem, the space step for leg $i$ is initialized to $\Delta x_{1}=z_{1} / 7$, where $z_{i}$ is the length of leg $i$. The next two criteria can only reduce or maintain these values for $\Delta x$, they will not increase them.

Courant Numbers. This criterion is based on the Courant number and is concerned with the numerical accuracy of NEFTRAN II. As discussed by Campbe11 and Longsine (1981), for the DVM transport model, numerical dispersion can be controlled by the magnitude of the Courant number. The Courant number is defined as 


$$
\mathrm{CN}=\frac{\mathrm{v} \Delta t}{\Delta \mathrm{x}}
$$

In general, to minimize numerical dispersion for the DVM transport model, CN should be greater than or equal to 1.0 for all important isotopes.

Since $\Delta t$ and $v$ are known, the $C N$ is set to 1.5 and $\Delta x$ is determined. This applies to both path-averaged and leg-to-leg transfer cases.

Source Length. For the path-averaged case, and if a flow-through source is being used, $\Delta x$ is constrained by the length of the source leg. Specifically, the space step cannot be greater than the length of the first leg since this determines the source blocks for the flow-through source model.

At this point, the largest permissible time step and spatial gridding have been selected within the constraints of the problem, based on the increment-determination parameters. Large values reduce computer execution time, which is desirable especially for statistical Monte Carlo simulations. In addition, large time steps also promote accuracy by limiting numerical dispersion. This is evident from the Courant number criterion above and is discussed in detail by Campbell and Longsine (1981). The apparent excessive attention to accuracy that is evident in the velocity model (Section 2.4.6), the discharge model (Section 2.4.7), the distribution of source into grid blocks (Section 2.4.8), and the legto-leg transfer model (Section 2.4.9) is required because of the large time steps that are used to limit numerical dispersion. For runs in which discharge of short-lived isotopes must be characterized, time steps should probably be smaller than that which is determined with the criteria discussed above and in Section 2.4.3. The reader is referred to Section 4.3.5 for suggestions to make the space- and time-step selection process more restrictive.

For simulations with steady-state velocity fields, the space and time steps found above are used throughout the entire problem simulation time. For simulations involving time-dependent velocity fields, a new space and time step are selected for each time interval as described above. An appropriate time step for each time interval is determined and stored. Space steps are found for each leg and the smallest space step is retained. Thus, the spatial gridding does not change from time interval to time interval. For all problem types, NEFTRAN II performs dimension checks for the number of grid blocks versus the size of arrays that contain atoms per grid block per isotope. If dimensions are exceeded, either $\Delta x^{\prime} s$ are increased and a warning message is written or the code aborts the run, depending on the severity of the excess.

\subsubsection{Courant Numbers and Isotope Travel Times}

The purpose of this section is to explain the importance of $C N$ and isotope travel time when simulating radionuclide transport with NEFTRAN II. With numerical transport models using finite-difference (FD) or finite- 
element (FE) methods, the accuracy of the transport simulation increases with decreasing time step. Thus, one can always improve accuracy with FD- or FE-based codes (at the expense of increased computer time) by simply decreasing the size of the time step. However, with DVM, numerical accuracy is generally improved by increasing the size of the time step. The reasons for this counter intuitive situation are explained by Campbell and Longsine (1981) and are not discussed here. The fact that DVM likes a large time step has the obvious benefit of reducing computer time (by factors of 10 or more over FD- and FE-based codes). However, this also implies that there is no trivial rule of thumb (such as decreasing the time step for FD or FE) for improving accuracy. The user should have the largest time step possible consistent with considerations of the time dependence of the source rate, isotope half lives, and desired resolution of the breakthrough curve. Because of the difficulty of finding the "optimum" space and time step for a given calculation, NEFTRAN II will automatically select a space and time step if the user simply enters zeroes for these values.

NEFTRAN II will usually do an adequate job of selecting a space step and time step. However, the user should generally print out space and time step information, as well as velocity model and retardation information in order to assure that reasonable numerical criteria are being met. The most important indicator of the numerical accuracy of NEFTRAN II is the $\mathrm{CN}$.

The general rule concerning the $\mathrm{CN}$ is that it should be greater than or equal to one for all important isotopes. As discussed in previous sections, an important isotope is one that is expected to discharge from its initial inventory during the problem simulation time. Conversely, an unimportant isotope is one that is not expected to discharge in significant amounts from its initial inventory during this time. In NEFTRAN II, for DVM transport, an unimportant isotope is one with travel time significantly larger than the problem simulation time or one with a half life less than one-tenth of the travel time. In the first case, the isotope will not discharge from its initial inventory because it will not reach the discharge point during the problem simulation time. In the second case, the isotope will decay to insignificantly low concentrations before discharging.

It is useful to examine the effects of $C N$ on numerical accuracy using NEFTRAN II. Figure 2.7 compares breakthrough curves generated using NEFTRAN II (DVM option) with an analytical solution. The travel time in this example is 500 years. The analytical solution is shown as a solid curve. NEFTRAN II results are shown for $\mathrm{CN}=0.1,1$, and 2 . For CN $=0.1$, substantial numerical dispersion is apparent, while for $C N=1$, the numerical dispersion is practically insignificant. The curve for $\mathrm{CN}=2$ is indistinguishable from the analytical solution. If the user is interested in the integrated discharge at 1000 years or the concentration (or dose) at any time after about 700 years, any of the three DVM curves would probably be adequate. However, suppose the user is interested in the concentration at 400 years. In this case, the most accurate curve between $\mathrm{CN}=1$ and $\mathrm{CN}=2$ would be used. To emphasize this point, 


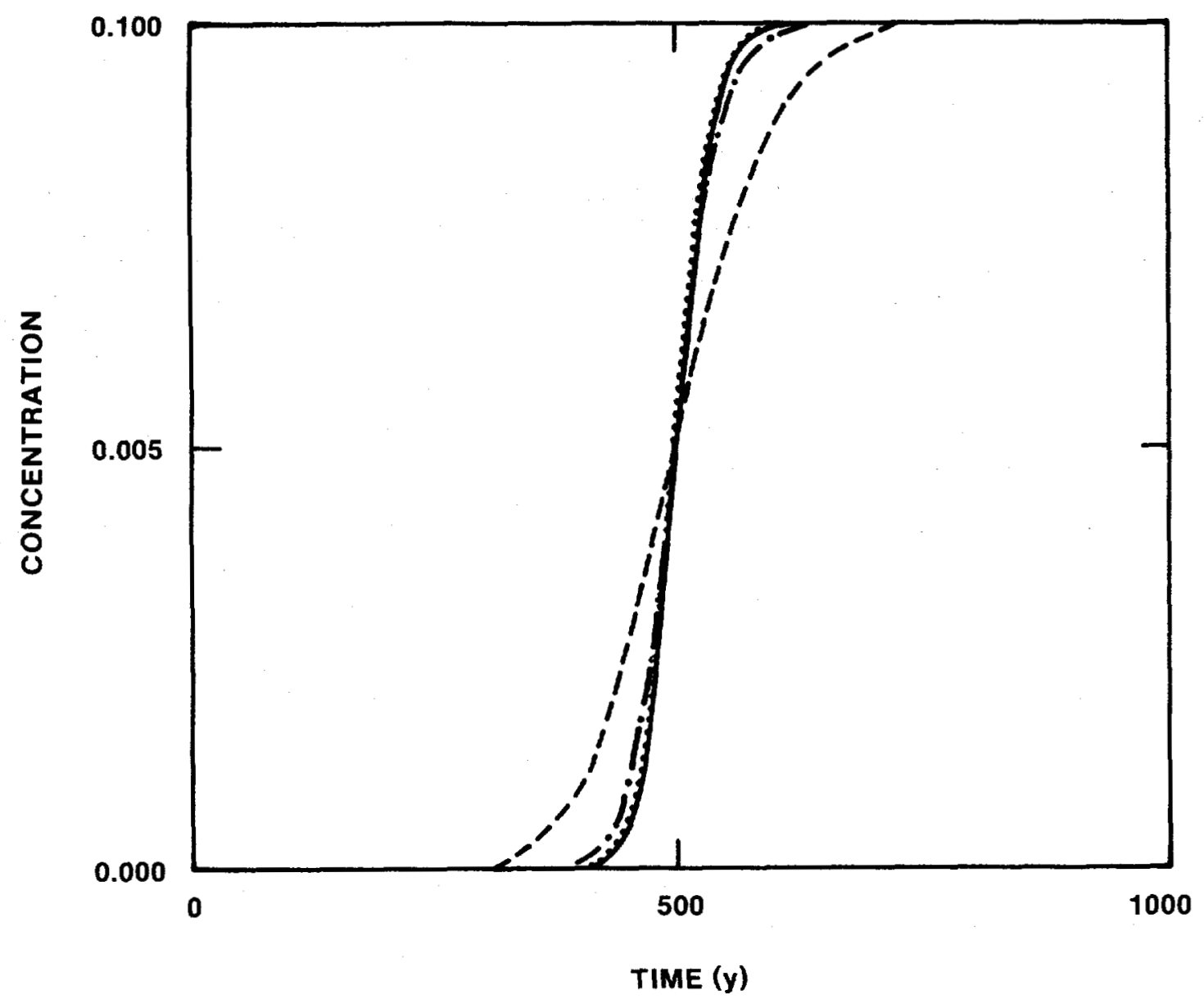

ANALYTICAL SOLUTION

$--\infty \quad$ DVM, CN $=0.1$

-.-. DVM, CN $=1.0$

DVM, $C N=2.0$

Figure 2.7. Example Breakthrough Curves (NEFTRAN II, Analytical Solution) 
consider Figure 2.8 which presents the same results as Figure 2.7 but with concentration plotted on a logarithmic scale. At 400 years, the concentration predicted using DVM with $\mathrm{CN}=2$ matches the analytical solution very well. For $\mathrm{CN}=1$, at 400 years, DVM overestimates the concentration predicted by the analytical solution by about a factor of 2 , while for $G N=0.1$, the over prediction is as much as three orders of magnitude. The message is that if concentration (or dose, etc.) at some time less than the travel time (when the discharge is greater than zero only because of physical dispersion) is the quantity of interest, it is very important that $\mathrm{CN}>1$. At later times, the values of $\mathrm{CN}$ is less critical, but should still always be greater than or equal to 1 for all important isotopes.

Figure 2.9 is included to help keep the above discussion in perspective. In this figure, the same analytical solution is compared to results generated using a finite difference transport code. The FD case with $\Delta x=20$ and $\Delta t=1$ shows about the same level of accuracy (or inaccuracy) as the earlier DVM case with $\mathrm{CN}=0.1$. The FD case in Figure 2.9 with $\Delta x=1$ and $\Delta t=0.5$ shows about the same accuracy as DVM with $C N=1$. However, the FD calculation took about 15 minutes compared to a few seconds with NEFTRAN II. No attempt was made with the FD code to generate results as accurate as the DVM case with $\mathrm{CN}=2$.

It should be apparent from the above discussion that the NEFTRAN II user not only needs to be aware of the value of $\mathrm{CN}$, but also the travel time in a given transport calculation. Before discussing travel time considerations, a few definitions are necessary.

The nominal or average isotope travel time is defined as:

$$
T_{n}=\frac{L}{v}
$$

where $T_{n}$ is the average isotope travel time, $v$ the average isotope velocity (ground-water velocity divided by isotope retardation factor in units of length/time), and $L$ the path length from the source to the discharge point.

In attempting to determine whether an isotope will discharge during the problem simulation time, one could compare $T_{n}$ with the problem simulation time. However, because of dispersion, discharge of an isotope begins some time before its average travel time. Thus, some approximation of the time of first isotope breakthrough is needed. To estimate this time, consider that the shape of the breakthrough curve near the average travel time is approximately that of an error function (i.e., the integral of a normal distribution). The standard deviation in time underlying the breakthrough curve can be estimated as

$$
\alpha_{T}=\sqrt{\frac{2 \alpha \mathrm{L}}{\mathrm{V}}}
$$




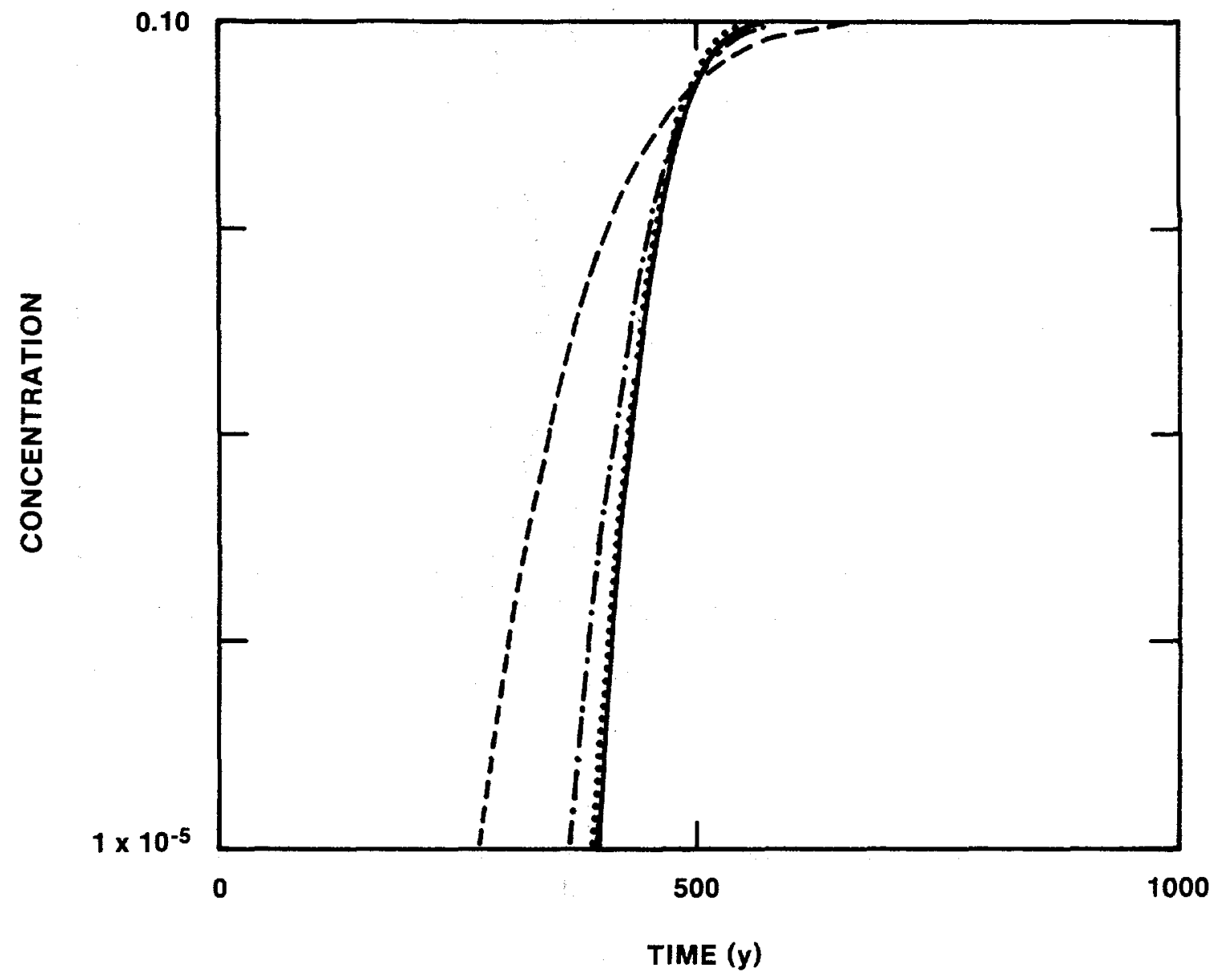

ANALYTICAL SOLUTION

DVM, $C N=0.1$

DVM, CN $=1.0$

DVM, $C N=2.0$

Figure 2.8. Example Breakthrough Curves with Concentration on a Logarithmic Scale 


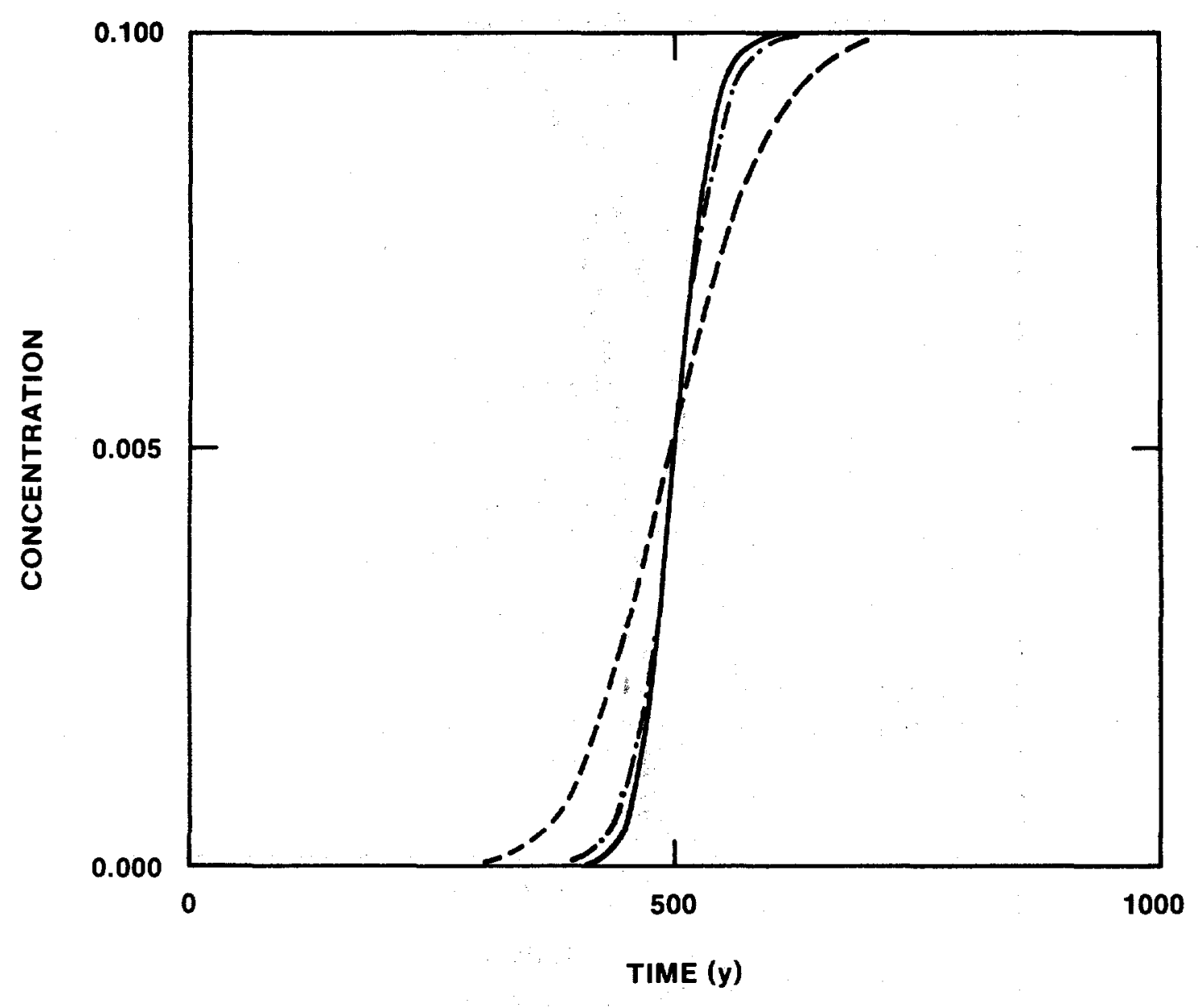

ANALYTICAL SOLUTION

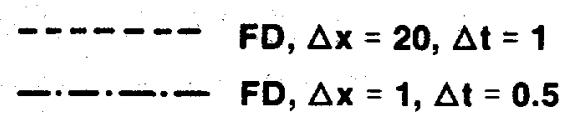

Figure 2.9. Comparison of Finite Difference Solution to Analytical Solution 
where $\alpha_{I}$ is the standard deviation in time, $\alpha$ the dispersivity (length), $L$ is the migration path length, and $v$ the average isotope velocity (length/time). The minimum travel time or the approximate time to first breakthrough is estimated as

$$
T_{m}=T_{n}-M \alpha_{T}
$$

where $T_{m}$ is the approximate time to first breakthrough, $T_{n}$ the average isotope travel time, and $M$ some multiplier to be determined.

Experience indicates that $M=2.3$ gives a good approximation to the time of first isotope breakthrough. The next example should help clarify the above ideas on travel time and isotope breakthrough.

For this example, consider the decay chain

$$
{ }^{243} \mathrm{Am} \rightarrow{ }^{239} \mathrm{Pu} \rightarrow 235 \mathrm{U} .
$$

The path length is 1000 feet, the dispersivity is 40 feet, and the fluid velocity is $0.1 \mathrm{ft} /$ year. The retardations, average isotope velocities, average isotope travel times, standard deviation in time, and approximate time to first breakthrough are given in Table 2.2.

Table 2.2

Parameters for Example Problem

\begin{tabular}{lccccc}
\hline ISOTOPE & RETARD. & $\mathrm{V}$ & $\mathrm{T}_{\mathrm{n}}$ & $\alpha_{\mathrm{T}}$ & $\mathrm{T}_{\mathrm{m}}$ \\
\hline Am243 & 150 & $6.67 \mathrm{E}-4$ & $1.50 \mathrm{E} 6$ & $4.24 \mathrm{E} 5$ & $5.25 \mathrm{E} 5$ \\
Pu239 & 15 & $6.67 \mathrm{E}-3$ & $1.50 \mathrm{E} 5$ & $4.24 \mathrm{E} 4$ & $5.25 \mathrm{E} 4$ \\
$\mathrm{U} 235$ & 75 & $1.33 \mathrm{E}-3$ & $7.50 \mathrm{E} 5$ & $2.12 \mathrm{E} 5$ & $2.62 \mathrm{E} 5$ \\
\hline
\end{tabular}

If $\mathrm{T}_{\mathrm{m}}>\mathrm{TUB}$, the isotope is not expected to discharge in significant amounts and is thus classified as "unimportant." For illustration, the example problem was run for problem times TUB of $1.0 \times 10^{5}, 5.0 \times 10^{5}$ and $1.0 \times 10^{6}$ years. For TUB $=1.0 \times 10^{5}$ years only ${ }^{239} \mathrm{Pu}$ should be considered important because $T_{m}$ for both ${ }^{243} \mathrm{Am}$ and ${ }^{235} \mathrm{U}$ are greater than TUB. For ${ }^{239} \mathrm{Pu}, \mathrm{CN}=1.35$, while for ${ }^{243} \mathrm{Am}$ and ${ }^{235} \mathrm{U}, \mathrm{CN}<1$. It is obvious that NEFTRAN II focused its space and time step considerations on ${ }^{239} \mathrm{Pu}$ as the travel times for the other two isotopes are too long to reach the discharge point during the problem simulation time. Figure 2.10 shows discharge rates for this case. As expected, ${ }^{239} \mathrm{Pu}$ begins to discharge at about 50000 years. However, ${ }^{235} \mathrm{U}$ also shows some discharge. This occurs 


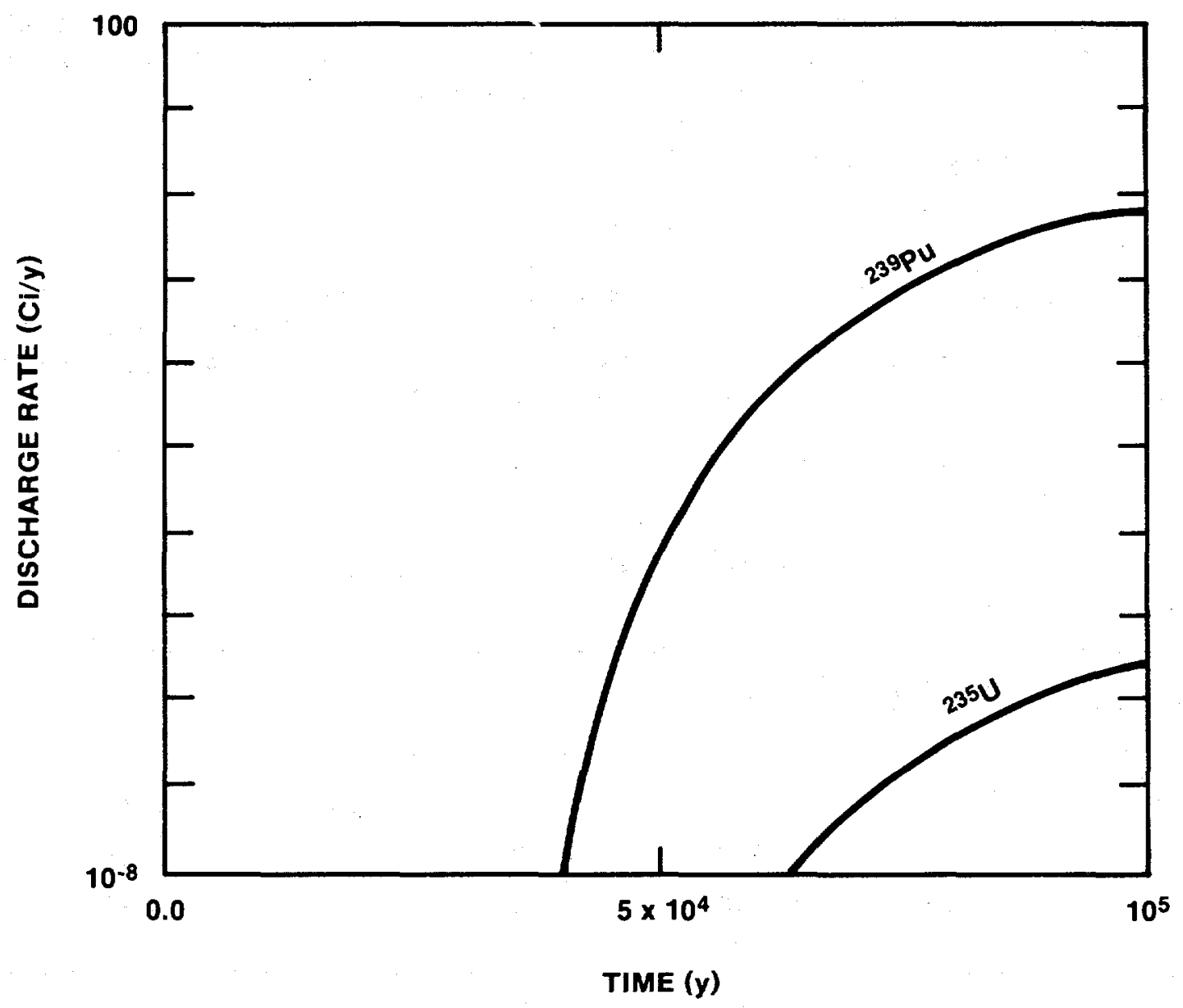

Figure 2.10. Discharge Rate Curves for Problem Simulation Time $=1.0 \times 10^{5} \mathrm{y}$ 
because ${ }^{239} \mathrm{Pu}$ decays along the migration path to ${ }^{235} \mathrm{U}$, some of which is then able to reach the discharge point. None of the original source of ${ }^{235} \mathrm{U}$ reaches the discharge point in this calculation.

The CN for TUB $=5.0 \times 10^{5}$ years are 6.8 for ${ }^{239} \mathrm{Pu}, 4.3$ for ${ }^{235} \mathrm{U}$ and less than $1(0.6)$ for ${ }^{243} \mathrm{Am}$. By referring to Table 2.2 , it is evident that both ${ }^{239} \mathrm{Pu}$ and ${ }^{235 \mathrm{U}}$ are expected to travel from the source to the discharge point during the problem time. Consequently, NEFTRAN II has increased the size of the time step so that both isotopes have $C N>1$. However, NEFTRAN II did not try to satisfy $\mathrm{CN}>1$ for ${ }^{243} \mathrm{Am}$ which has a minimum travel time slightly greater than 500000 years. Discharge curves for this case are shown in Figure 2.11. ${ }^{239} \mathrm{Pu}$ again breaks through at approximately the expected time, while ${ }^{235 \mathrm{U}}$ produced by decay of ${ }^{239} \mathrm{Pu}$ begins discharging at approximately this time also. Note that at approximately $2.5 \times 10^{5}$ years, ${ }^{235} \mathrm{U}$ discharge begins to increase. This corresponds to arrival of the original source inventory of ${ }^{235} \mathrm{U}$.

CN for TUB $=1.0 \times 10^{6}$ years for all three isotopes are $>1$. The discharge rate curves for TUB $-1.0 \times 10^{6}$ years are shown in Figure 2.12 . Note than ${ }^{243} \mathrm{Am}$ does not discharge in $1.0 \times 10^{6}$ years even though its minimum travel time is about $5.0 \times 10^{5}$ years. The reason is that the half-life of ${ }^{243} \mathrm{Am}$ is only $7.37 \times 10^{3}$ years so that all the initial inventory for this isotope has decayed before it reaches the discharge point. The reason that ${ }^{243} \mathrm{Am}$ was apparently considered important (i.e., CN $>1$ ) is that NEFTRAN II was able to produce $C N>1$ for the important isotopes ( ${ }^{239} \mathrm{Pu}$ and ${ }^{235 \mathrm{U})}$ and meet other numerical criteria by simply using the default minimum number of time steps (i.e., the default maximum time step size). This default time step just happened to be large enough to give $\mathrm{CN}>1$ for ${ }^{243} \mathrm{Am}$.

It is not always possible for NEFTRAN II to achieve $C N>1$ for all important isotopes. In the above example, the retardation factors, and hence, the isotope velocities, vary one order of magnitude. Consequently, the largest $C N$ is a factor of 10 larger than the smallest. Suppose the velocities varied over three orders of magnitude. If the slowest isotope were "important" (i.e., expected to discharge) and NEFTRAN II were to select $\Delta \mathrm{x}$ and $\Delta t$ such that this isotope had $\mathrm{CN}>1$, then the fastest isotope would have $\mathrm{CN}>1000$. That is, the fastest isotope would jump 1000 grid blocks each time step. The time step is limited such that the migration path cannot be traversed in one time step and, therefore, to obtain a CN > 1000, more than 1000 grid blocks would be required. For NEFTRAN II, the dimensions are limited to 1000 grid blocks and it would not be possible to satisfy $\mathrm{CN}>1$ for the important isotope in this example without exceeding dimensions. A compromise $\Delta x$ and $\Delta t$ would have to be selected or the dimensions increased. Alternatively, should such a case occur in practice, the user may want to appeal to the physics of the problem. For example, could the very slow moving isotope be left in the source calculation to affect solubilities but not transported? If the isotope is at the bottom of a chain, can it be simply ignored or just take on the Courant number of its parent if it would only discharge with its parent isotope? Another alternative might be to break the decay chain at the slow moving isotope if it is sufficiently long-lived. 


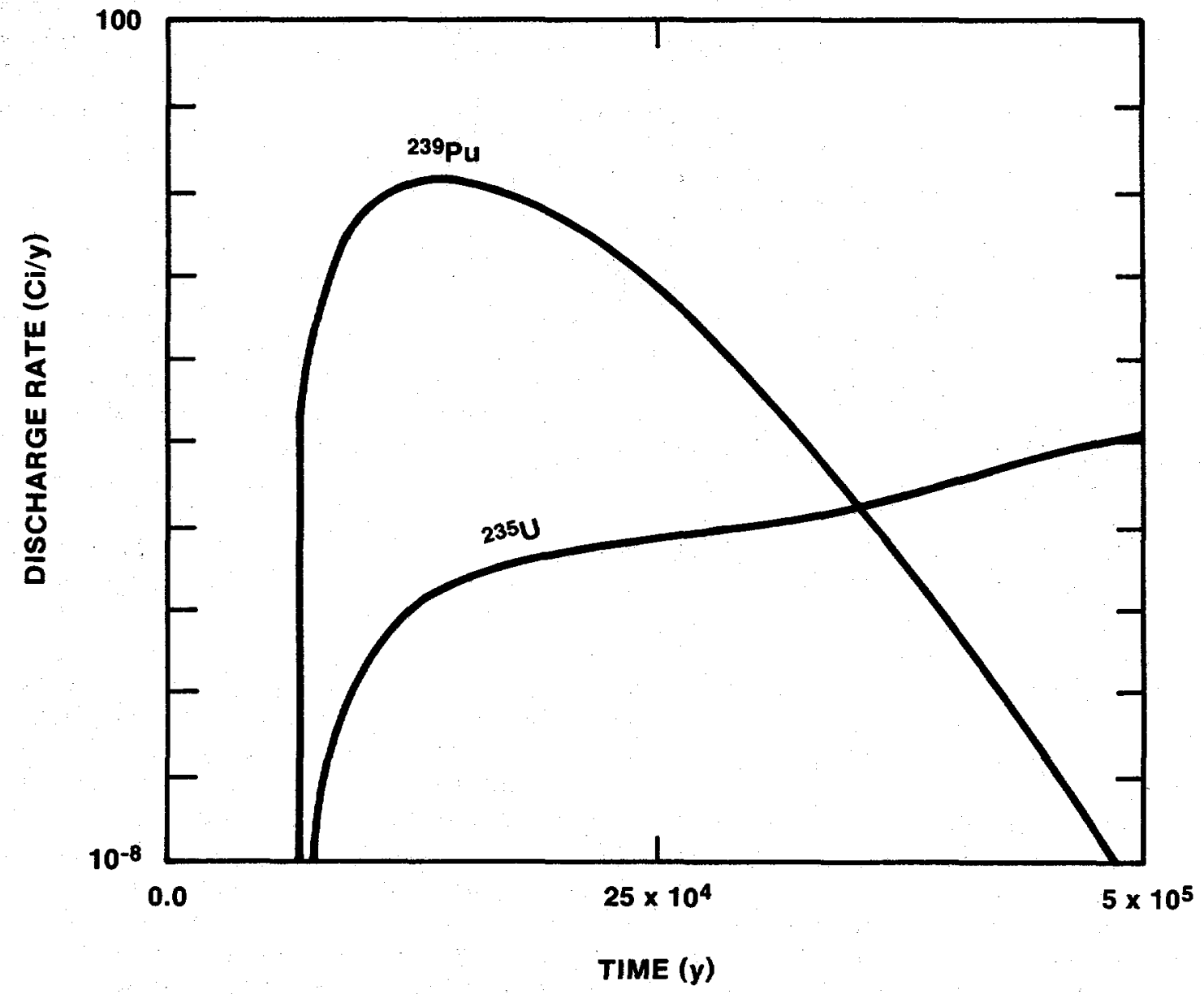

Figure 2.11. Discharge Rate Curves for Problem Simulation Time $=5.0 \times 10^{5} \mathrm{y}$ 


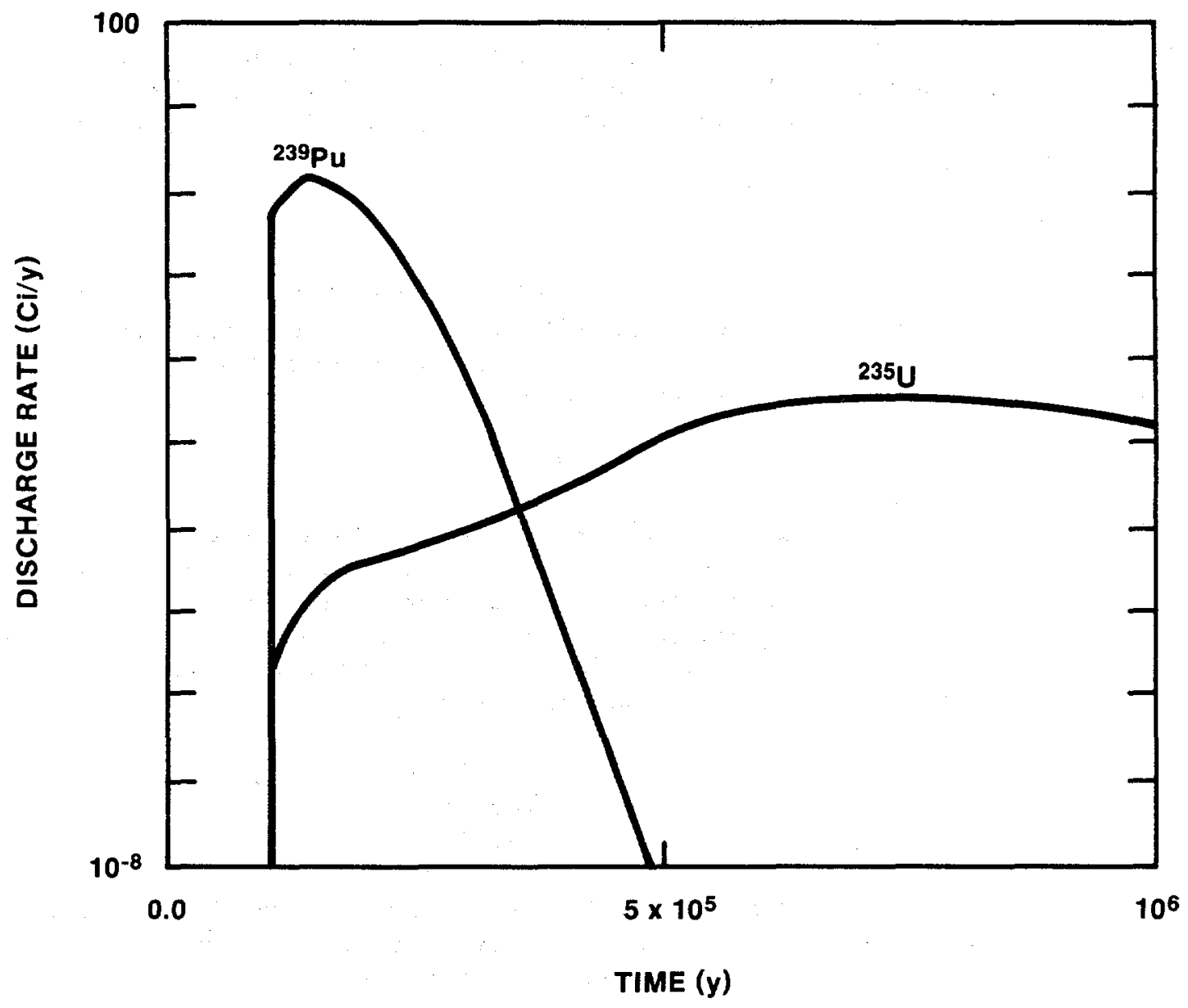

Figure 2.12. Discharge Rate Curves for Problem Simulation Time $=1.0 \times 10^{6} \mathrm{y}$ 
In summary, to get accurate discharge rate results using NEFTRAN II, the user must be aware of isotope travel times, half lives, and $\mathrm{CN}$. In general, CN for important isotopes should be $>1$. Important isotopes are those with half lives sufficiently long ( $>10 \%$ of the minimum travel time) and velocities sufficiently large (minimum travel time < problem simulation time) that they are expected to discharge from their initial inventory during the problem simulation time. In most cases, it is probably not necessary to estimate a minimum travel time but simply to examine the isotope velocities provided by an optional NEFTRAN II output file. As a final summary point, the user must recognize the inherent difficulty in trying to transport chains that have a very large range of velocities. In this case, Courant numbers for the slowest isotopes may have to be compromised. In most realistic cases, however, these isotopes will have such long travel times that they will be classified as unimportant.

\subsubsection{Species Velocity Model}

As indicated in Section 2.4.2, application of the DVM transport model to a radioactive decay chain requires a method for determining the average velocity of atoms that decay to daughter products during a time step, $\Delta t$. As an example, consider the simple decay chain

$$
\mathbf{A} \rightarrow \mathbf{B}
$$

and suppose that $A$ and $B$ have different retardation factors and therefore, different average velocities. To transport all the atoms in a particular grid block, it is necessary to separately transport those A atoms that survive decay during $\Delta t$, atoms of isotope $A$ that decay to $B$ during $\Delta t$, and finally, atoms of isotope $B$ that survive decay during $\Delta t$. That is, one must transport 3 "subchains" namely,

$$
\begin{array}{ll}
A \rightarrow A & \text { velocity } v_{A} \\
A \rightarrow B & \text { velocity } v_{A B} \\
B \rightarrow B & \text { velocity } v_{B}
\end{array}
$$

As velocities $v_{A}$ and $v_{B}$ are assumed known, the problem is to calculate $v_{A B}$, the average velocity for atoms of isotope $A$ that decay to $B$ (and remain as $B$ to $\Delta t$ ) during $\Delta t$. One can write $v_{A B}$ as

$$
v_{A B}={ }_{A}^{-T_{A}} v_{A}+(\Delta t-\bar{T})_{B}
$$

where $\bar{T}_{A}$ is the average time spent as $A$ for atoms that decay to $B$ during $\Delta t . \quad \bar{T}_{A}$ is found by writing the probability that, in time interval $\Delta t$, species A decays to $B$ which survives to the end of $\Delta t$. This probability is written as: 


$$
D P(A \rightarrow B)=\left(e^{-\lambda_{A} t}\right]\left(\lambda_{A} d t\right)\left[e^{-\lambda_{B}(\Delta t-t)}\right) .
$$

The first term in parentheses is the probability that species A survives to time $t$. The second term is the probability that $A$ decays to $B$ during infinitesimal time dt. The last term is the probability that $B$ survives decay to the end of $\Delta t$. Thus, the probability that species A decays to species $B$ over $\Delta t$ is an integral of the product of independent probabilities. To find $\bar{T}_{A}$, the integrand is weighted by $t$ and the resulting integral is divided by $\operatorname{DP}(A \rightarrow B)$. This is equivalent to

$$
\overline{\mathrm{T}}_{\mathrm{A}}=\frac{\int_{0}^{\Delta t} t \lambda_{A} e^{-\lambda_{A} t} e^{-\lambda_{B}(\Delta t-t)} d t}{\int_{0}^{\Delta t} \lambda_{A} e^{-\lambda_{A} t} e^{-\lambda_{B}(\Delta t-t)} d t} .
$$

The solution of Equation 2.85 is

$$
\bar{T}_{A}=\frac{e^{\left(\lambda_{b}-\lambda_{a}\right) \Delta t}}{e^{\left(\lambda_{b}-\lambda_{a}\right) \Delta t}-1} \Delta t-\frac{1}{\lambda_{b}-\lambda_{a}}
$$

Substitution of Equation 2.86 into 2.83 yields $\mathrm{v}_{\mathrm{AB}}$. This approach is extended to decay chains of several isotopes in Appendix A.

The implementation of DVM is based on the transport of subchains within a given decay chain. For example, suppose the decay chain $I_{1} \rightarrow I_{2} \ldots \rightarrow I_{5}$ is to be transported. In DVM, 15 subchains are potentialiy transported over each $\Delta t$. These subchains are

$$
\begin{aligned}
& I_{1} \quad I_{1} \rightarrow I_{2} \quad I_{1} \rightarrow I_{2} \rightarrow I_{3} \quad I_{1} \rightarrow I_{2} \rightarrow I_{3} \rightarrow I_{4} \quad I_{1} \rightarrow I_{2} \rightarrow I_{3} \rightarrow I_{4} \rightarrow I_{5} \\
& \mathrm{I}_{2} \quad \mathrm{I}_{2} \rightarrow \mathrm{I}_{3} \quad \mathrm{I}_{2} \rightarrow \mathrm{I}_{3} \rightarrow \mathrm{I}_{4} \quad \mathrm{I}_{2} \rightarrow \mathrm{I}_{3} \rightarrow \mathrm{I}_{4} \rightarrow \mathrm{I}_{5} \\
& I_{3} \quad I_{3} \rightarrow I_{4} \quad I_{3} \rightarrow I_{4} \rightarrow I_{5} \\
& \mathrm{I}_{4} \quad \mathrm{I}_{4} \rightarrow \mathrm{I}_{5} \\
& I_{5}
\end{aligned}
$$


Column 4, for example, includes the subchains necessary to update the amounts and locations of species $I_{4}$. However, the contribution to species $I_{4}$ over a single time step from species $I_{1}$ and (possibly) species $I_{2}$ may be insignificant due to the relative half-lives of each species. If this is the case, it is reasonable to transport only $I_{3} \rightarrow I_{4}$ and $I_{4}$. Past experience indicates that restricting subchains to a maximum length of five is adequate. Encountering the need to transport a six-member subchain is not anticipated and, in fact, has never been noted. However, when subchains of length 6 or greater are encountered, an approximate scheme, presented in Appendix $A$, is used.

\subsubsection{Discharge Model}

Determining total discharge of species $r$ from $t$ to $t+\Delta t$ is based on evaluation of

$$
\delta(r, t, \Delta t)=\sum_{\mathrm{p}=0}^{\mathrm{q}} \sum_{i=1}^{\mathrm{NB}(\mathrm{r}, \mathrm{r}-\mathrm{p})} \mathrm{F}(\mathrm{r}, \mathrm{r}-\mathrm{p}, \mathrm{i}) \rho\left(\mathrm{r}-\mathrm{p}, \mathrm{N}_{\mathrm{x}}-\mathrm{i}+1, \mathrm{t}\right),
$$

where $N B(r, r-p)$ marks the grid block farthest from the boundary that contains precursor $r$ - $p$ and can contribute to the discharge of $r$, $F(r, r-p, i)$ is the fraction of species $r-p$ in grid block $N_{x}-i+1$ that discharges as species $r$, and $\rho\left(r-p, N_{x}-i+1, t\right)$ is the number of atoms of species $r-p$ in grid block $N_{x}-i+1$ at time $t$.

The determination of array $F$ (and as a by-product, the array NB) is quite complicated and is the subject of Appendix $B$. As seen by the notation, $F$ is independent of time. Hence, $F$ can be constructed during the setup process and stored. The apparent excessive attention to accuracy that is evident in determining the $F$ array is necessary due to the large time steps that are used to limit numerical dispersion for DVM.

For the leg-to-leg transfer option (see Section 2.4.9) particles are not allowed to skip a leg over a time step, thus only particles in the last leg can discharge over a time step. By replacing the path-averaged velocities with the velocities of the last leg, the process of finding discharge fractions for the leg-to-leg transfer option is identical to the leg-averaged option described in Appendix $B$.

\subsubsection{Distribution of Source Into Grid Blocks}

The source model determines the total amount of source for each isotope over time that is available for transport. In the flow-through source model, all material available for transport for each transport time step is injected evenly into the source leg grid blocks, where the source leg is the first leg in the migration path. Consequently, for transport, NEFTRAN II requires at least two legs in the migration path; the first leg contains the source blocks and all subsequent legs the transport path. 
Distribution of source particles into the grid blocks using a mixing-cell source is quite complicated and is described in detail in Appendix $C$. The basic premise is that particles leaving the mixing cell are given a velocity using DVM as they exit the mixing cell, and allowed to travel as far as they will go over the time remaining in the transport time step. As particles exit the mixing cell into the migration path, the scheme takes into account radioactive decay and differing species velocities. Similiar to DVM transport and discharge, sets of fractional multipliers are developed for grid blocks that receive source particles over a time step.

\subsubsection{Leg-to-Leg Transfer Mode1}

Section 2.4.2 discusses the implementation of the DVM transport model based on the assumption of a single one-dimensional homogeneous leg representing the entire migration path for radionuclides. In others words, even though each leg in the migration path may represent a different medium, the velocities in each leg are averaged and the DVM transport model is applied to the averaged velocity with a single dispersivity. For saturated porous media, combining different velocities into a single average velocity has been shown to yield acceptable results and to be computationally efficient (Campbell and others, 1981). However, if some of the legs are to represent fractured media with the accompanying diffusion into the rock matrix, the contact time between fluid in the fracture and matrix becomes important. The contact time induced by using a velocity averaged over both porous and fractured legs may not be physically meaningful. Also, to simulate transport in both unsaturated and saturated media, the dispersivity for each zone may be significantly different such that using DVM based on a single dispersivity may not be appropriate. Therefore, the DVM transport model has the capability to include the concept of transporting radionuclides between different legs over a time step (i.e., leg-to-leg transfer). Within each leg, the DVM concept of distributing velocities is implemented as discussed in Section 2.4.2.

The simulation of leg-to-leg transfer of radionuclides must account for particles that begin a time step in one leg and end the time step in another. For the leg-to-leg transfer model, the magnitude of the time step is restricted such that a leg cannot be skipped over the time step (see Section 2.4.3). Consequently, particles with a given velocity that begin the time step in a certain leg are assumed to either end the time step in that leg or in the next leg. As mentioned previously, with only one exception (discussed at the end of this section), particles that do not exit a leg in a time step are accounted for in the same way as described in Section 2.4.2.

Leg-to-leg transfer is complicated by the possible occurrence of negative velocities in the distribution of velocities for each leg. Negative velocities can occur due to the assumption that solute dispersion can be modeled assuming a Gaussian distribution of velocities. This is the conventional treatment of dispersion and is a property of the mathematical formulation, not the physical process. The physical process of 
mechanical dispersion (1.e., excluding molecular diffusion) can only result in movement of material downstream in the direction of flow and not upstream. For the leg-to-leg transfer model, if negative velocities are encountered, particles are not allowed to travel backwards into the previous leg in the migration path. This is accomplished by attaching "catcher" blocks at the leading edge of each leg. The use of catcher blocks in the leg-to-leg transfer model will be discussed at the end of this section.

The occurrence of negative velocities causes one further difficulty for implementing the DVM leg-to-leg transfer model. It requires that the code track the leading and trailing edges of a contributor block forward in time to see if there is any overlap with the given receiver block. This is different than the implementation of DVM for the path-averaged case, where the code tracks backwards in time from receiver blocks to find all possible contributor blocks.

The need for this altered approach is due to the possibility that contributor blocks may be undefined for some receiver blocks. In other words, every receiver block in a new leg may not have a source block from the previous leg. This happens when a packet of particles ends up straddling a leg boundary. If the velocity of the particles is less than zero in the first leg and greater than zero in the second leg, the original contents split into two separate packets. Consequently, the partial packet that travels backwards in the first leg does not contribute to the expected receiver block in the second $\mathrm{leg}$ and the source for these receiver blocks becomes undefined. Thus, it is easier in the leg-to-leg transfer model to track particles forward in time rather than to search backwards for contributor blocks.

Intuitively, the logical time to check when a packet may straddle a boundary should be at the end of a time step. However, due to radioactive decay, the time when a packet may straddle the leg boundary is checked after the time spent as each isotope. This time is discussed in Section 2.4.6. For example, for the subchain $I_{1} \rightarrow I_{2} \rightarrow I_{3}$, the time the packet is straddling the leg boundary after $T_{1}$, the time spent as $I_{1}$, is significant.

Before discussing the scheme for tracking packets forward in time in the leg-to-leg transfer model, the general set up of NEFTRAN II will be discussed. A packet is defined as the fraction of material in a contributor block that begins the time step as isotope $i, I_{i}$, and ends the time step as isotope $p, I_{p}$. For example, the decay chain $I_{1} \rightarrow I_{2} \rightarrow I_{3}$ will have six packets in each contributor block corresponding to the subchains:
1) $\mathrm{I}_{1} \rightarrow \mathrm{I}_{1}$
2) $\mathrm{I}_{1} \rightarrow \mathrm{I}_{2}$
3) $\mathrm{I}_{2} \rightarrow \mathrm{I}_{2}$
4) $\mathrm{I}_{1} \rightarrow \mathrm{I}_{2} \rightarrow \mathrm{I}_{3}$ 
5) $\mathrm{I}_{2} \rightarrow \mathrm{I}_{3}$

6) $I_{3} \rightarrow I_{3}$

The fraction of material contained in a packet, $b$, is

$$
f_{b}=M_{k} \operatorname{DP}\left[I_{1} \rightarrow I_{p}, t\right] \text {, }
$$

where $M_{k}$ is the material in contributor block $k$ and $D P\left[I_{i} \rightarrow I_{p}\right.$, $\left.t\right]$ is the decay production factor.

For the leg-to-leg transfer model, to track a packet forward, the progress of the packet as species $I_{1}$ for time $T_{1}$ is first tracked. Then the packet is tracked as it migrates with the velocity of species $I_{i+1}$ for time $T_{i+1}$ and so forth. If packet $b$ ends $T_{i}$ with both leading and trailing edge in the same leg, the location for packet $b$ is updated and stored. If the packet ends $T_{i}$ straddling a leg boundary, the packet is split into two new packets and relevant information is stored. Each new packet is characterized by:

a. the location of its leading edge,

b. the location of its trailing edge, and

c. the fraction (geometric mixing fraction) of the original packet that the new packet represents.

At the end of the time step, it is determined whether any of the packets, old or new, overlap receiver blocks. This information is important in order to find the appropriate geometric mixing fractions for the potential receiver blocks.

To find the geometric mixing fractions for contributions from blocks in leg $L$ to receiver blocks in the next leg $(L+1)$, the leg-to-leg transfer model accounts for the following four different overlap possibilities (illustrated in Figure 2.13):

a. both the leading and trailing edges of packet $b$ are located in receiver block $i$,

$b$. the leading edge of packet $b$ is in receiver block $i$, but the trailing edge is not.

c. the trailing edge of packet $b$ is in receiver block $i$ and the leading edge is not, or

d. the leading and trailing edges of packet $b$ envelope receiver block $i$.

If none of the conditions (a) - (b) are true then there is no overlap and the geometric mixing fraction for receiver blocks in the next leg is zero. 


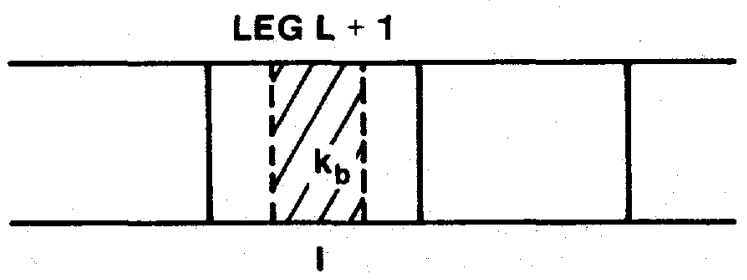

(a)

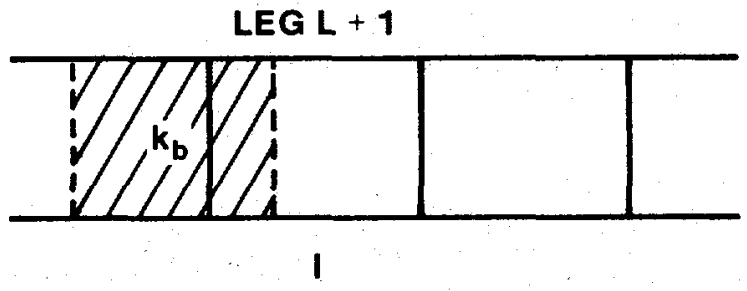

(b)

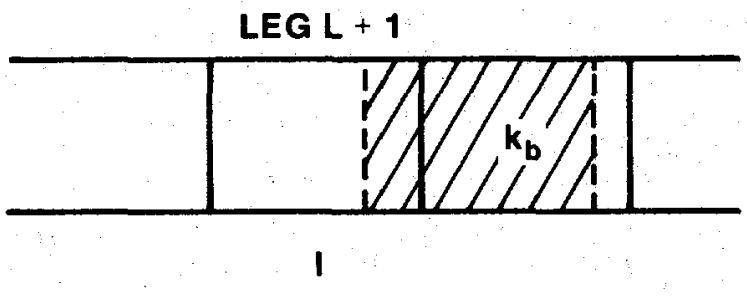

(c)

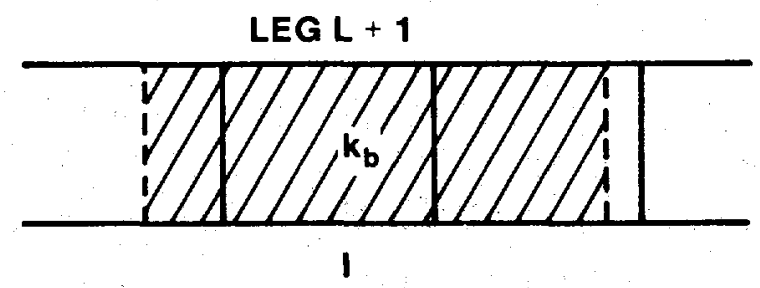

(d)

Figure 2.13. Illustration of Four Possible Situations Arising Because of an Overlap of a Packet and a Given Receiver Block 
As indicated previously, another topic that needs to be addressed for leg-to-leg transfer is when a negative velocity dictates movement from leg $L$ to leg $\mathrm{L}-1$. To account for these negative velocitles, the leg-toleg transfer model artificlally extends the length of leg $I$ to "catch" the negatively directed particles. Figure 2.14 shows three such catcher blocks appended to leg $\mathrm{L}$. Particles in leg L- 1 have a velocity distribution calculated with Equation 2.59 using the pore velocity, dispersivity and retardation of $\mathrm{leg} \mathrm{L}-1$. As these particles arrive at the boundary between leg L-1 and leg L, they are subject to the velocity distribution in leg $\mathrm{L}$ corresponding to the pore velocity, dispersivity and retardation of leg $L$. The velocity distribution in leg $L$ may have negative components that will force particles backwards into leg L-I. However, this is not allowed because once particles leave leg L-1 they are no longer transported with the properties of that leg. Instead, catcher blocks are added to the entry of leg $L$ to allow particles to move backwards and be transported with the velocity distribution of leg $L$ without actually going back into leg L-1. After each time step, the particles that are in the catcher blocks are summed and put into the first grid block of leg $\mathrm{L}$. This ensures that no particles are allowed to migrate farther back in the artificial catcher blocks.

There is a potential inconsistency in the treatment of particles near a leg boundary. This inconsistency can occur for a velocity packet in which the parent velocity is positive and the daughter velocity is negative. An example is a two member chain for which this occurs for at least one packet. In this case the packet splits and part of the original packet remains in leg $L$ and part remains in leg $L+1$. However, for transport in the interior of $\operatorname{leg} \mathrm{L}$, the fraction contributing to the last grid block is larger than what it is supposed to be. In fact, the entire distance from the trailing edge of the packet to the leg boundary is available for interior transport since the packet maintains the velocities for leg $\mathrm{L}$ throughout the time step. Thus, not allowing particles to return to their original leg can result in double counting by the interior transport fraction near the boundary.

To correct for the above special case, an algorithm was developed to adjust the amounts of particles that remain in each leg. For each subchain that has more than one member, the need for adjustment is checked. If a velocity interval is detected having some positive movement followed by some negative movement, the contributions from block to block in the leg interior near the down-gradient boundary are traced. If necessary, an adjustment fraction is generated and saved. This algorithm is also applied to the last leg to avoid inconsistent treatment with the discharge model discussed in Section 2.4.7.

\subsubsection{Matrix Diffusion Mode1}

NEFTRAN II contains a model that treats the exchange of radionuclides between a mobile dynamic fluid and an immobile stagnant fluid in conjunction with the DVM transport model. An example of where this model may be applicable is in simulating radionuclide transport from fractures to the surrounding rock matrix. The expected effect of this phenomenon on the 


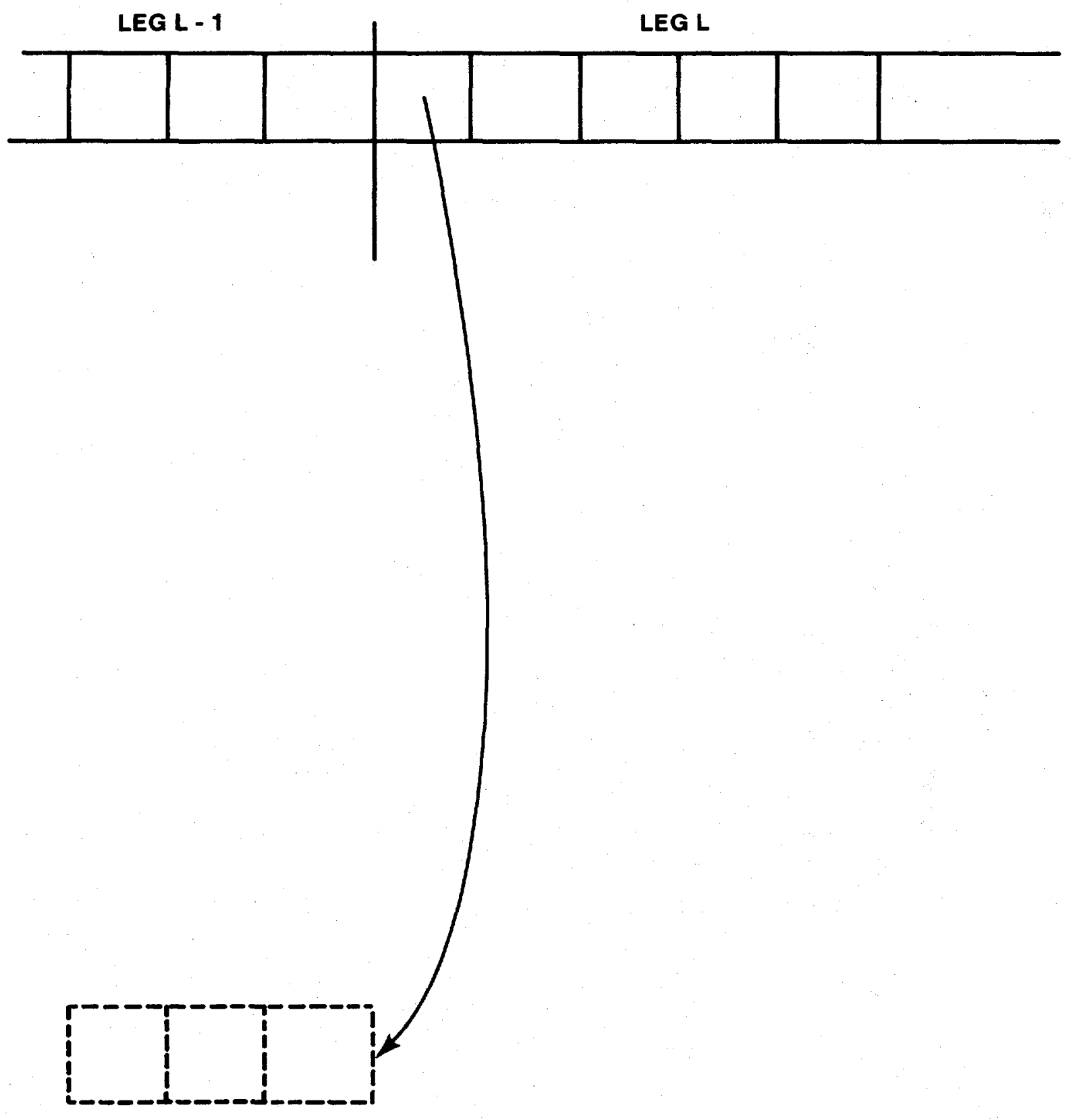

Figure 2.14. Illustration of Catcher Blocks Appended to Leg L 
transport of stable species is to cause dispersion of the species. For decaying species the retention period in the immobile phase may be long enough to reduce concentration through radioactive decay significantly. This model may also be applicable for simulating transport in porous media that contains dead-end pores.

Theory. The matrix diffusion model contained in NEFTRAN II is based primarily on the work of van Genuchten and Wierenga (1976). The model uses the simplifying assumptions of a constant immobile pore volume to represent the immoble phase and a mass exchange coefficient to determine the mass flux between the mobile phase and the immobile phase.

The rate of diffusion from the mobile phase to the immobile phase is assumed to be directly proportional to the concentration difference between the mobile and immobile phases. Letting subscript d (dynamic) denote mobile phase properties and subscript s (stagnant) denote immobile phase properties, the governing mathematical equation for the rate of change of concentration in the immobile phase is

$$
\theta_{s} R_{s} \frac{\partial C_{s}(x, t)}{\partial t}=\beta\left(C_{d}(x, t)-C_{s}(x, t)\right)
$$

where $C$ denotes concentration in units of mass per unit volume and the constant of proportionality, $\beta$, is the mass exchange coefficient with units of inverse time, $\theta$ is the volume fraction occupied and $R$ the retardation factor.

Implementation. As discussed above, van Genuchten and Wierenga (1976) account for the loss of solute from the mobile fluid to the immobile fluid by the addition of a sink term to the convective-dispersion equation. Since time is discretized in DVM, this fracture-matrix exchange can be included at the end of each time step. That is, particles are transported in the mobile phase using the conventional DVM solution of the convective-dispersion equation. At the end of a transport step, concentrations in the mobile phase are compared to those in the immobile phase and diffusional exchange is modeled.

For those legs along the migration path that the user selects as representing dual-porosity media, a parallel system of grid blocks is generated. The only mechanism for molecules to move from one system to the other is by diffusion. Figure 2.15 shows 1 eg $L$ with its associated immobile phase. As indicated in Figure 2.15, the $\mathrm{K}^{\text {th }}$ block of $\operatorname{leg} \mathrm{L}$ is associated with the $K^{\text {th }}$ block containing immobile fluid for leg $L$. Thus, exchange occurs between pairs of blocks in the two systems. No exchange occurs between adjacent immobile phase blocks.

For each block pair, the rate of diffusion is assumed to be directly proportional to the concentration difference between the mobile and immobile phases, as indicated in Equation 2.89. A forward finitedifference approximation of the time derivative in Equation 2.89 yields 


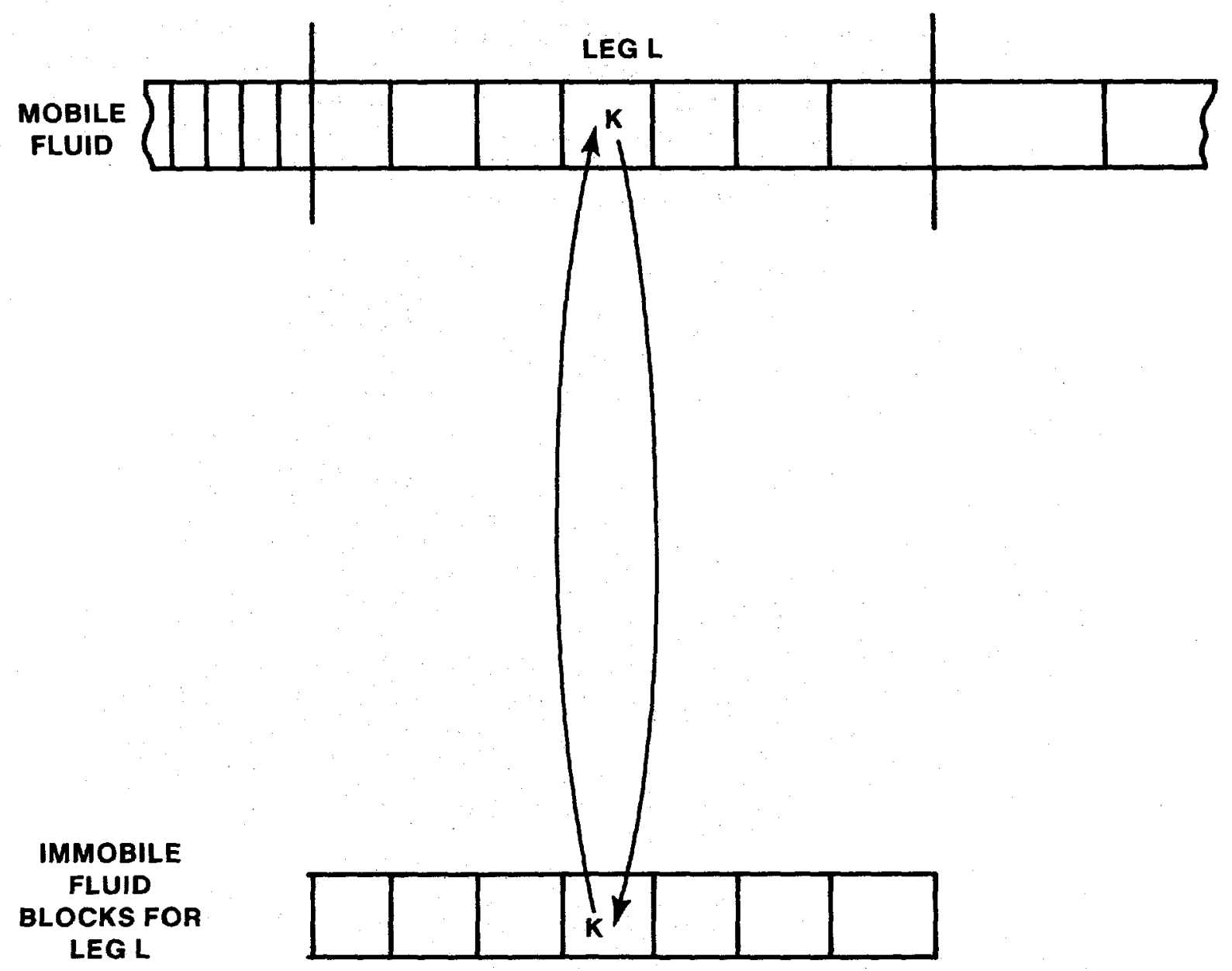

Figure 2.15. Illustration of Adjacent System of Grid Blocks Simulating the Rock Matrix in Dual-Porosity Legs 


$$
C_{s}(x, t+\Delta t)=\frac{\beta}{\theta_{s}} \frac{\Delta t}{R_{s}}\left[C_{d}(x, t)-C_{s}(x, t)\right]+C_{s}(x, t)
$$

Incorporation of Equation 2.90 into DVM requires that particles in a given grid block are converted into concentrations. Allowing for retardation, this conversion is as follows

$$
\left.\begin{array}{l}
c_{d}(x, t)=\frac{P_{d}(x, t)}{\theta_{d} R_{d}} \\
c_{s}(x, t)=\frac{P_{s}(x, t)}{\theta_{s} R_{s}}
\end{array}\right\},
$$

where $P(x, t)$ is the quantity (in atoms) of the given species at location $x$ and time $t, R$ is the retardation factor and $\theta$ is the volume fraction occupied.

Using Equation 2.90 and Equation 2.91, the number of atoms in the stagnant region at $t+\Delta t$ is

$P_{s}(x, t+\Delta t)=\left\{\frac{\beta}{\theta_{s}} \frac{\Delta t}{R_{s}}\left[C_{d}(x, t)-C_{s}(x, t)\right]+C_{s}(x, t)\right\} \theta_{s} R_{s}$.

To complete the solution, the concentration in the dynamic fluid must also be updated. This is done by conserving mass (atoms) between the immobile and mobile phase before and after diffusional exchange. That is

$$
\mathrm{P}_{\mathrm{d}}=\mathrm{P}_{\mathrm{T}}-\mathbf{P}_{\mathrm{S}}
$$

where $P_{T}$ is the total number of atoms in the system (mobile plus immobile) before diffusional exchange.

After $P_{d}$ is found, the value of $P_{s}(x, t+\Delta t)$ is updated to account for radioactive decay. The spatial distribution of particles in the mobile phase at time $t+\Delta t$ is calculated using the approaches discussed in Section 2.4.2.

To summarize, the implementation of the fracture-matrix exchange algorithm is as follows:

(i) transport radionuclides in the mobile phase from $t$ to $t+\Delta t$,

(ii) calculate exchange for each nuclide using Equations 2.90 through 2.93, 
(iii) in preparation for the next time step, age the atoms in the immobile fluid to $t+2 \Delta t$,

(iv) set $t=t+\Delta t$ and return to (i).

These steps are repeated until an upper bound time is reached. Step (iii) insures that radionuclides aged to the same time in both phases are interchanged.

The implementation of step (ii) uses Equations 2.90 through 2.93 with location $x$ governed by grid block number. There is one added complication, however. Since the time steps for DVM are typically large, straightforward use of Equation 2.90 can result in concentrations modified beyond the point of equilibrium. Thus, NEFTRAN II requires the concentration difference between the fractures and the matrix not to change sign over $\Delta t$. So if, for example,

$$
C_{d}>C_{s}
$$

and after accounting for diffusional exchange, it is found that

$$
\mathrm{C}_{\mathrm{d}}<\mathrm{C}_{\mathrm{s}}
$$

then exchange should have ceased when equilibrium was reached. The equality

$$
C_{d}=C_{s},
$$

is imposed whenever this occurs.

\subsubsection{DVM Transport in Time-Dependent Velocity Fields}

The most significant difference between NEFTRAN II and NEFTRAN is the NEFTRAN II capability to transport radionuclides in time-dependent velocity fields. Specifically, NEFTRAN II simulates time-dependent velocity fields by approximating the time-varying velocities with sequential steady-state velocities. The user defines time ranges over which the velocity in each leg is essentially constant and these velocities are read into NEFTRAN II from an external file (see Section 4.3.1). As discussed in Section 2.4.2, the only difference in implementation of DVM for time-dependent velocities is following each time interval, the code recomputes the transport fractions based on the new velocities and new time steps to be used for the next time interval.

This capability was added to NEFTRAN as a first approximation to modeling transport in unsaturated media. For unsaturated-zone transport, transport parameters become time dependent. For example, saturation is a 
function of time and, therefore, the retardation factor and velocity, as they are a function of saturation, are also time dependent. Although radionuclide transport in the unsaturated zone is complex and may not be completely defined at this time, this first approximation may be used to mimic the effects of transport in the unsaturated zone, and still retain the efficiency of the code needed for performance assessment requirements. It is also possible that, for certain combinations of parameters, this approximation is acceptable.

Besides modeling unsaturated-zone transport, this capability can also be used to model significant climatic changes that result in ground-water velocity changes. For example, one could assume that, over a period of 10000 years, the climate changes at 5000 years such that the flow velocities are doubled. However, the basic underlying assumption is that the flow paths do not change with time (i.e., the migration path is constant with time), and only the magnitude of the velocity is time-dependent.

Time-dependent Saturation, As indicated above, for transport in the unsaturated zone, the retardation factor is a function of saturation $\left(\mathrm{n}_{\mathrm{s}}\right)$ which is time-dependent. To be able to mimic this effect, NEFTRAN II allows the user to input time-dependent saturations along with timedependent velocities. The only effect that saturation has is on the retardation factor ( $R$ ), where the unsaturated retardation factor is given by

$$
R_{\text {unsat }}=n_{s} R \quad .
$$

This is a first approximation to the effects of the unsaturated-zone transport on retardation; however, it would be simple to incorporate another relationship, not necessarily based on saturation, as long as the effect on retardation is linear.

Besides being used to model unsaturated-zone transport, this capability could be used to mimic a linear time-dependent geochemical effect on retardation. For example, if the retardation factor was assumed to be a linear function of mineral composition and, the composition was depleted with time, this capability could be utilized. 
The following sections will describe how NEFTRAN II is structured, as well as the subroutines, the parameter statements and the common blocks contained in the code.

\subsection{Structure}

The code structure will first be described in general, including the structure that is followed for multiple simulations. Next, the structure of the source module and the transport module contained in NEFTRAN II will be discussed.

\subsubsection{General}

A flow chart of the general structure of NEFTRAN II is shown in Figure 3.1. The first task that is executed by the main program is to call a subroutine to read the input file(s) provided by the user. Next, if the user has requested for NEFTRAN II to solve the fluid flow network, the network flow model is implemented by calling the appropriate subroutines. The next function of the main program is to implement the source model contained in NEFTRAN II. The source model provides the source rates that are required by the DVM transport model, but is independent of the DVM transport model. The final step in the simulation is radionuclide transport. For multiple chain simulations, the DVM transport model is implemented independently for each chain. After all chains have been transported, the last task of the main program is to call a subroutine to write the discharge rates for each isotope to the appropriate output files.

Automated multiple simulations in NEFTRAN II can be accomplished by two different means. First, a completely new input file or parts of the input file can be read for each new simulation based on certain input options (see Section 4.5.1). Consequently, if the user wanted to run multiple simulations for completely or partially different input sets, one could simply append all of the input sets into one input file, with the appropriate options indicated between each data set. NEFTRAN II would execute each data set until an end-of-file was encountered.

Second, specific input can be over-written based on data from an external file (see Section 4.3.1 for a description of this file). This is termed the statistical Monte Garlo run mode of NEFTRAN II, where the external file contains vectors of randomly sampled input variables found from a computer code like LHS (Iman and Shortencarier, 1984). Each vector contains one value for each of the set of sampled data input variables. For the statistical run mode, NEFTRAN II reads input data from the basic input file, and if the user has requested for sampled data to be read from an external file, it replaces the input data parameters with values from the external file. This is repeated for each sampled vector until an end-of-file is encountered in the external file. The reader should note that these two ways of conducting multiple simulations with NEFTRAN II are not mutually exclusive and although not normally done, both could be implemented at the same time if this was required by the user. 


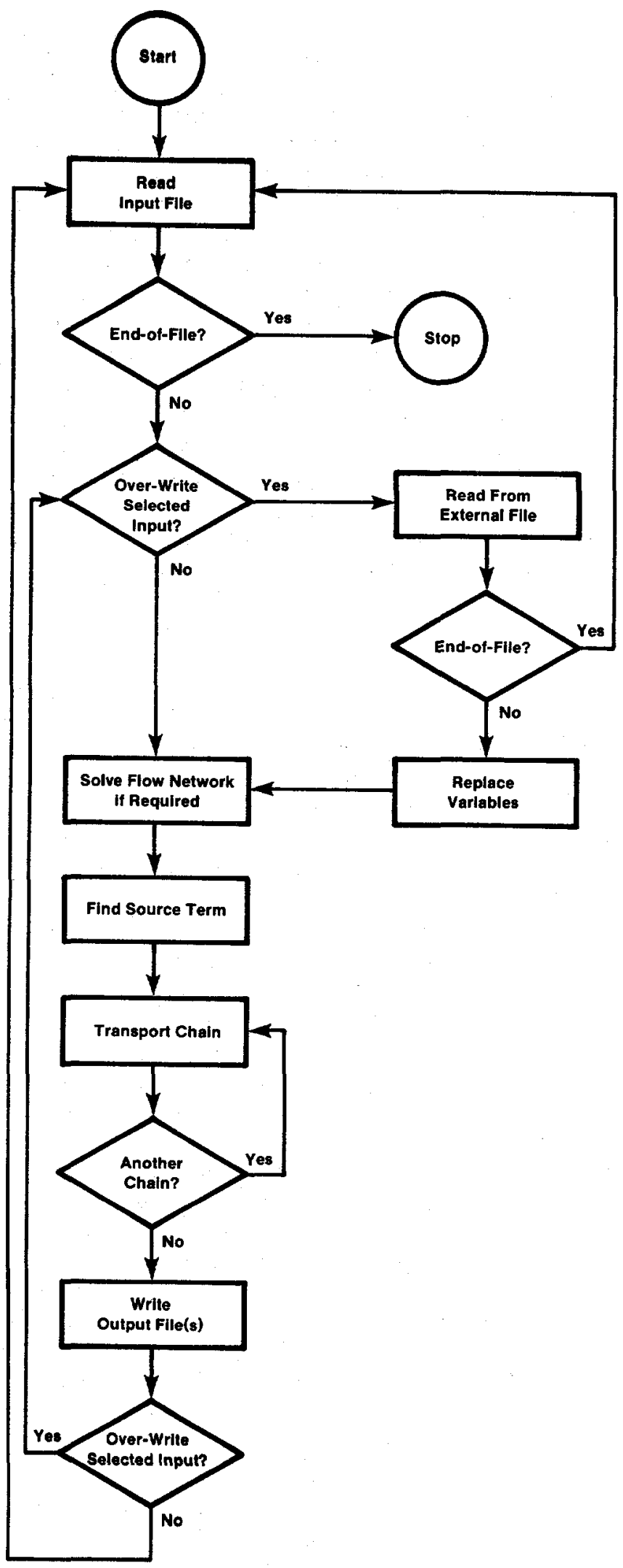

Figure 3.1. General Flowchart of NEFTRAN II 


\subsubsection{Source Module}

Figure 3.2 shows a flowchart of the source module contained in NEFTRAN II. The module loops through time based on source time steps. The first process that is accounted for over a time step is leaching. If leaching is the only process being considered (as specified by the user) then the source rate can be determined based on the amount leached for either the flow-through source or the mixing-cell source, whichever model is being implemented. However, if the solubility-limit of the source is also being modeled, then the amount leached is added to the undissolved inventory. Next, if there is a time-dependent flow rate through the source, the total flow rate over the time increment is found. This is needed in the next step where the amount dissolved, based on solubility limits is found. Next, a comparison between the amount dissolved based on solubility limits and the amount left in the inventory is made.

The amount placed in the dissolved inventory is the minimum of these two quantities. This amount dissolved is then subtracted from the undissolved inventory. At this point the source rates can be found for either the flow-through source or the mixing-cell source, again, depending on which model is being utilized. After the source rates are found, the inventory is updated for the next time step, and the time is incremented. If the incremented time is greater then the problem simulation time, the source module terminates its calculations.

\subsubsection{Transport Module}

Figure 3.3 presents a flowchart of the transport module contained in NEFTRAN II. If the analytical solution is being implemented for simulating transport, the appropriate equations (Equation 2.18 - Equation 2.22) are solved. If the DVM transport model is being utilized to solve for transport, for time-dependent velocities, transport is simulated for the first time interval. If the fluid velocities are not time-dependent, only one time interval occurs and this interval is from the release time to the problem simulation time. Before the simulation actually occurs, the transport fractions can be found. If the mixing-cell source model is being implemented, the fractions relating the distribution of the source in the transport path can be found as well as the leg-to-leg transfer fractions, if the leg-to-leg transfer algorithm is being implemented. Finally, before actually simulating transport, the discharge fractions are found. During the simulation, for each time step, matrix diffusion is accounted for if the user has indicated that some of the legs in the transport path are dual-porosity legs. The transport simulation is terminated when the time is greater than the time interval. If the time interval is greater than or equal to the problem simulation time, the transport module calculations are stopped. Otherwise, the transport fractions are found for the next time interval and transport is simulated for that time period.

\subsection{Subroutines}

The following sections will briefly describe the function of each subroutine contained in NEFTRAN II, as well as where the subroutine is called from and what subroutine(s) it calls. The first subroutine that 


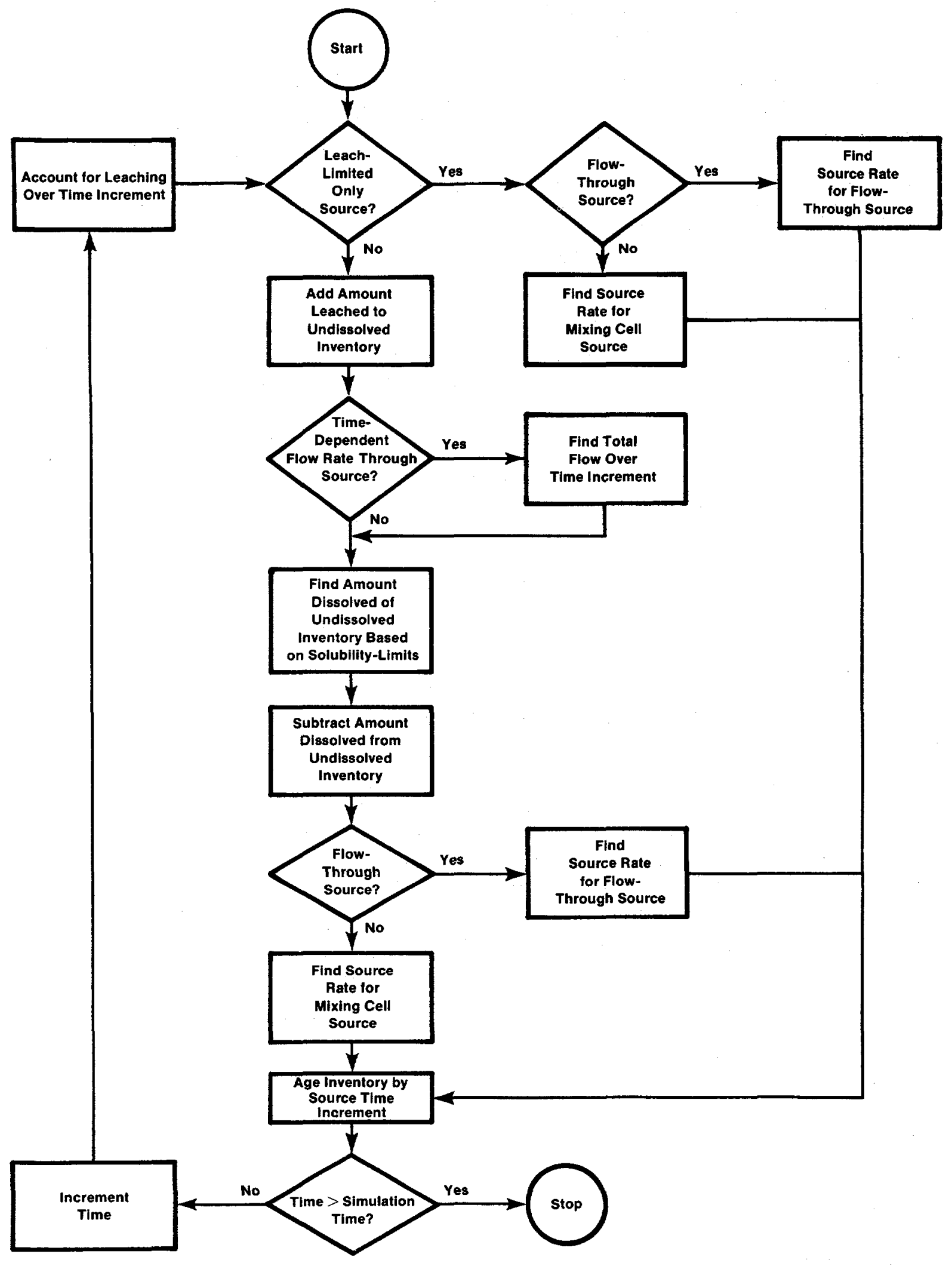

Figure 3.2. Flowchart of Source Module in NEFTRAN II 


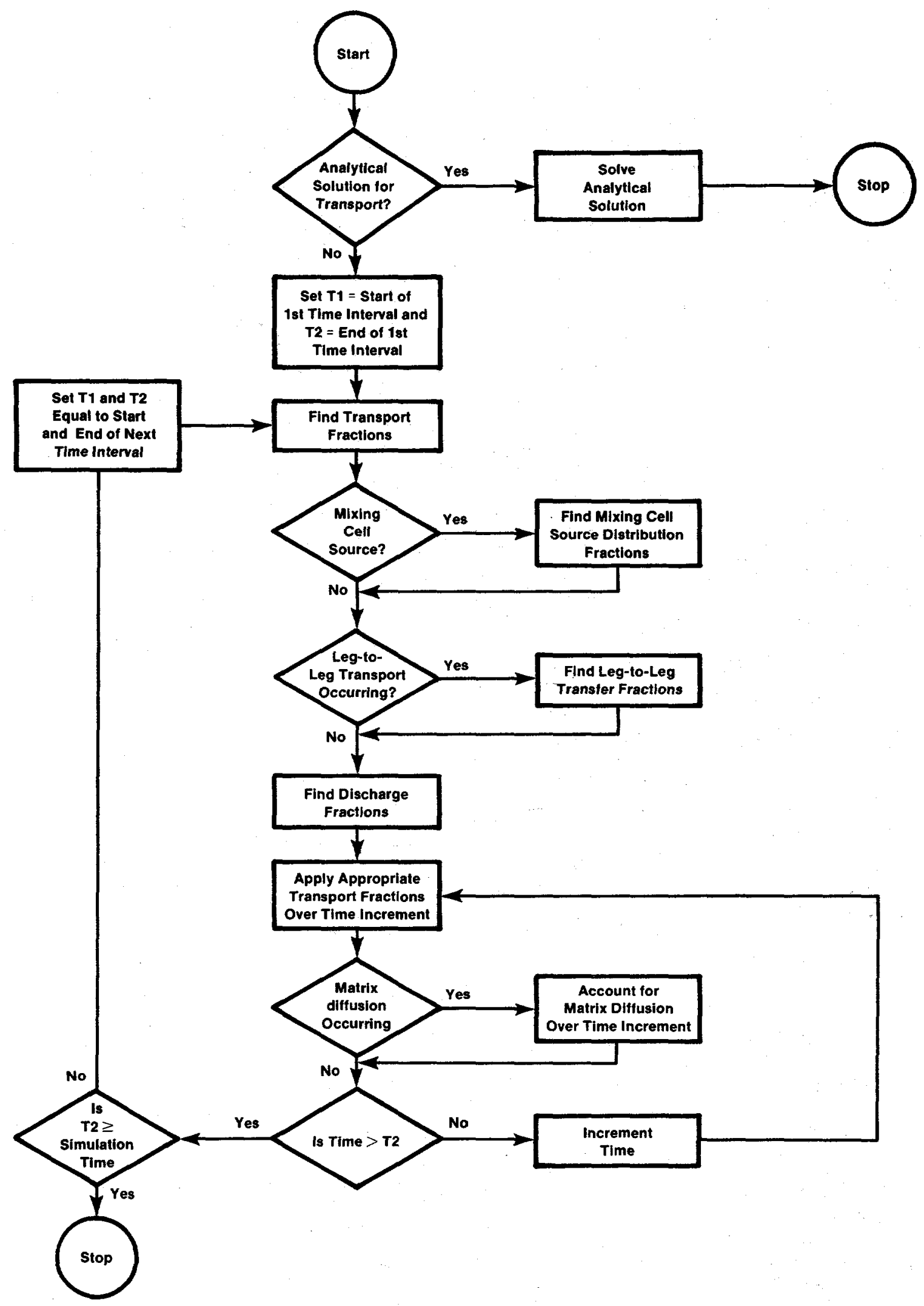

Figure 3.3. Flowchart of Transport Module in NEFTRAN II 
is discussed is the main driving program (NEFMAIN) and subsequent subroutines are discussed in alphabetical order.

\subsubsection{NEFMAIN - main program}

Called From

Calls

-...........

BSOLVE, CHAIN, COEFF, FLOWIN, GETRV, METHOD, PTHLEN, SOURCE, WORK

NEFMAIN is the main driving program of NEFTRAN II. It is capable of handling single or multiple simulations. It calls FLOWIN in which the data input is read. For multiple runs, the initial call to FLOWIN serves as the basic set up. Subsequent runs can be accomplished by either calling FLOWIN for additional data sets or GETRV to read input vectors from an external file (unit 10) and overwrite existing data (for a discussion of this external file see section 4.3.1). If the user opts for the flow network to be solved, NEFMAIN calls COEFF and BSOLVE, checks an error flag to see if BSOLVE was successful in solving the pressure equations, and condenses the pressure array since some of the pressures are known from input. NEFMAIN calls PTHLEN to calculate pore fluid velocities if the flow network has been solved, or to check the user input velocities for flow direction consistency. These velocities are required by the transport model. Next, NEFMAIN implements the source model by calling SOURCE. The source model is decoupled from the transport model and provides source rates for the DVM transport model. If the analytical transport option is chosen, SOURCE updates the user-specified inventories to release time and exits. For each chain, NEFMAIN calls CHAIN and METHOD to implement the transport model. After the transport simulations are completed for each chain, NEFMAIN calls WORK to write discharge information to the appropriate output file (unit 30).

3.2.2 ADJB(VA, LG, IP, TS, ILAST, KNT)
Called From
Calls
FACER
-....

Subroutine ADJB is implemented with the DVM transport mode1 in conjunction with the leg-to-leg transfer option. When the leg-to-leg transfer algorithm is invoked in FACER, negative velocities (see Section 2.4.9) can cause double counting of some particles at the end of each leg. Subroutine ADJB is called from FACER to compute the fractions to correct for this double counting for each leg.

\subsubsection{BAND (TSTART, TEND)}
Called From
Calls
METHOD
GIT, RATIO, TPPRT

Subroutine BAND is called from METHOD when the user opts for the analytical transport model (see Equation 2.18 - Equation 2.22). BAND applies the analytic solution for a chain of up to three radionuclides with equal retardation factors at a prescribed distance from the source. The source 
boundary condition is a point source in space and a decaying band in time.

\section{2 .4 BRANCH (EPS)}

Called From

SOURCE, DTUPDT, SETUP
Calls

ET

Subroutine BRANCH creates the radioactive decay subchains that are used to estimate decay/production of an isotope over a time step and calls ET to find the decay/production factors (as far upchain as necessary) based on the Bateman equations. SOURCE calls BRANCH to update the radionuclide inventory to the release time, DTUPDT calls BRANCH to age the inventory over the initial source time step, and SETUP calls BRANCH to age the inventory by the DVM transport time step.

In creating the subchains, BRANCH traces branches from an isotope upchain to the end of the chain(s). The current code logic allows four such paths. BRANCH determines how far upchain to look for reasonable representation of the production of the isotope over a time step using ratios of Bateman production/decay factors. EPS is the maximum cutoff ratio. When called from SOURCE, EPS is $10^{-8}$, when called from DTUPDT, EPS is $10^{-5}$, and when called from SETUP, EPS is 10-4. Terminating a subchain prior to a branch point decreases the number of necessary paths. For a detailed discussion of how NEFTRAN II stores decay chain information, which is implemented in subroutine BRANCH, see Appendix D.

\subsubsection{BSOLVE(KER)}

Called From NEF

Calls -....

Subroutine BSOLVE is called from NEF when the network flow model is implemented. BSOLVE solves the banded matrix equation set up in subroutine COEFF using a Gaussian elimination technique. This subroutine precludes the need to use an external subroutine package to solve the matrix equation.

\subsection{6 $\mathrm{CATCH}(\mathrm{GA}, \mathrm{NPT}, \mathrm{VDT})$}

Called From $\quad$ Calls
DXDT
$-\cdots$

For the DVM transport model, subroutine CATCH determines the maximum distance (VDT) that will be required to catch negative directed particles for each leg. DXDT and CHAIN use this distance and the space step to determine the total number of catcher blocks needed for each leg.

\subsubsection{CHAIN(IW)}

Called From NEF, SOURCE
Calls

DXDT, TRACER 
Subroutine CHAIN substitutes information for a given chain in a multiplechain execution to local arrays. When called from SOURCE (IW $\neq 0$ ), CHAIN calls TRACER to find the mean travel times for each isotope which are used by DTUPDT to determine isotope importance for source time step determination. When called from NEF (IW - 0), CHAIN calls TRACER to determine mean travel times to be used by DXDT for transport time step determination. For the DVM transport model, CHAIN also calculates the number of catcher blocks needed for each leg. For time-dependent velocities, CHAIN saves the minimum space step required and the maximum distance required to catch negatively directed particles for each leg for all time frames, and checks to make sure dimensions are not exceeded based on these parameters.

\subsubsection{CHKPTH}

Called From $\quad$ Calls
PTHLEN
$-\ldots$.

Subroutine CHKPTH is called by PTHLEN to verify that flow is downgradient along the user-input migration path. It also checks that legs along the migration paths are sequentially connected.

\section{2 .9 COEFF}

Called From Calls
NEF

COEFF is called when the network flow model is implemented and puts into matrix form the system of equations used to solve the flow network (see Section 2.1). Specifically, COEFF creates the transmissivity matrix and the constant vector used in solving for the unknown pressures at leg junctions. COEFF also implements the viscosity submodel.

\subsubsection{DTUPDT}
Called From
Calls
SOURCE
BRANCH, ET

DTUPDT provides the time step for the source model calculations if it is not input by the user. Section 2.3.2 presented the basic criteria for time-step determination and discussed the specific parameters that DTUPDT applies in finding the time step for source calculations. By calling BRANCH and ET, DTUPDT also finds subchains and decay/production factors over the source time step.

\section{2 .11 DXDT}
Called From
Calls
CHAIN
CATCH

DXDT finds the space and/or time step for the DVM transport model if they are not input by the user. Material is presented in sections 2.4 .3 and 2.4.4 that discusses the basic criteria for space- and time-step determination and discusses the structure of DXDT and how it applies the set of 
criteria to determine the time and space increment. DXDT calls CATCH to find the maximum distance that will be required to catch negative directed particles for each leg.

\section{2 .12 ET}

$\begin{array}{ll}\text { Called From } & \text { Calls } \\ \text { SETDIS, BRANCH, DTUPDT } & \text { RATIO }\end{array}$

Function ET is used to evaluate the Bateman coefficients for calculating the radioactive decay/production factors. When called from BRANCH, ET finds the factors for either updating the source inventory to release time or for updating the inventory by the DVM transport time step or the source time step. When called from SETDIS, ET finds the decay/production factors for isotopes surviving decay to discharge. When called from DTUPDT, ET finds the decay/production factors over one half the source time step. ET uses a Taylor's series expansion to solve for the Bateman coefficients. If convergence of the Taylor's series is expected to require too much execution time (i.e., a combination of small half-lives and large time-step), ET calls RATIO to evaluate the Bateman coefficients in a pairwise manner.

\subsubsection{FACER}

$\begin{array}{ll}\text { Called From } & \text { Calls } \\ \text { SETUP } & \text { ADJB }\end{array}$

Subroutine FACER implements the leg-to-leg transfer algorithm for the DVM transport model. FACER finds the leg interface fractions for each isotope in each subchain crossing from a leg to the subsequent one. For velocity packets that are negatively directed, FACER calls ADJB to adjust the fractions to ensure that double counting does not occur at the leg interfaces.

\subsubsection{FLOWIN(IPASS, ROOT, KROOT)}

Called From

NEF
Calls

INPRIN, STOPPER

Subroutine FLOWIN reads in all of the data input required by NEFTRAN II, with the exception of GETRV (see next section). For multiple data sets, IPASS $>1$, FLOWIN re-reads only the input indicated by the INP array (see Section 4.5.1), and if an end-of-file is encountered it is assumed to signify a normal termination. On the first pass (IPASS $=1$ ), FLOWIN opens the input and output files, centers the title and finds the execution date and time. For chains that are not included in the source calculations, (see Section 4.3.2), FLOWIN condenses the appropriate arrays. FLOWIN also calls STOPPER to do general data input checking.

For the PC version of NEFTRAN II, ROOT is a character string input by the user for the name of the input file (\{root\}.INP, unit 2) not including the extension of the file name. ROOT is then used by FLOWIN to access other input files if required and to name output files accordingly. 
KROOT is a counter that is used to count how many characters are contained in the character string ROOT. See section 4.1 for a more detalled discussion of the file naming scheme for NEFTRAN II.

\title{
3.2.15 GETRV(JTRIAL)
}

\author{
Called From Calls \\ NEF $\quad$ STOPPER
}

GETRV is used in performing multiple simulations for statistical studies which allows the user to assign selected sampled values to the appropriate NEFTRAN II variable names. Consequently, for the most part, GERTV is a user-written subroutine and the user is responsible for the required replacement statements and units conversion factors. GETRV reads the external file (unit 10), which contains each statistical trial vector. Simulations are conducted automatically for each vector, where the selected input is over-written with the vector values for each simulation based on the user-supplied replacement statements. JTRIAL is read from the external file for each vector, and indicates the trial or vector number. An end-of-file encountered in the external file indicates normal termination for a statistical run. The format for the external file is discussed in Section 4.3.1.
$3.2 .16 \mathrm{GIT}(\mathrm{T}, \mathrm{Y}, \mathrm{V}, \mathrm{AL})$
Called From
Ca11s
BAND
-...-

Function GIT is called from BAND and uses a fifth-order Hastings approximation (Abramowitz and Stegun, 1965) to calculate the sum of the complementary error function terms given in Equation 2.22. The complete analytical transport model solution is generated in BAND.

\subsubsection{INPRIN(MM, INCRM)}

Called From

Calls

FLOWIN

-...-

INPRIN prints user opted input parameters to the hardcopy output file. Some of the parameters may have been altered in subroutine STOPPER due to input error checks, otherwise they are as input. $M$ M indicates which parameter sets the user has designated to be printed and INCRM is a flag which indicates whether or not the default values are utilized for the space- and time-step determination parameters. INCRM equal to zero indicates that the default values have been used.

3.2.18 INTG(S1, B1, S2, B2, T1, T2, D, F, N, DT, ANS )

Called From

Calls

MXCLL

......

Subroutine INTG is called from MXCLL and is accessed only when the mixing-cell source is used. INTG is used to evaluate the integrals in the mixing-cell source model developed in Appendix $C$. The variables in 
the argument list are the limits of integration that are found in MXCLL and defined in Appendix $C$.

\subsubsection{LECHMOD(T1, T2, F)}

Called From Calls

SOURCE

LECHMOD is part of the source model and is called from SOURCE. LECHMOD finds the total fractions unleached at times $\mathrm{T} 1=t$ and $\mathrm{T} 2=t+\Delta t$. The fraction, F, leached is the difference of these two fractions. Depending on user input, the fraction leached is either based on a constant leach rate or an exponentially decaying leach rate.

\subsubsection{METHOD}

$\begin{array}{ll}\text { Called From } & \text { Calls } \\ \text { NEF } & \text { BAND, SETUP, TPPRT, TRNSPT }\end{array}$

Subroutine METHOD is the driving subroutine for the transport module. If the user selects the analytical transport model, METHOD sets the start time, stop time and time-step and calls BAND to solve the analytical transport model equations (Equation 2.18 - Equation 2.22). See Section 2.2.2 for a discussion on how the starting time, stopping time and timestep are found. Before calling BAND, METHOD checks to see if discharge occurs during the problem simulation time based on the average migration time for each isotope. If no discharge is detected then METHOD calls TPPRT to print out zero discharge information to the appropriate output files at the release time (TRLSE) and the problem simulation time (TUB). For the analytical solution, METHOD also checks to see if the last rate is written at a time less than the problem simulation time. If this is true, METHOD calls TPPRT to print/append zeroes to the appropriate output files.

If the user opts for the DVM transport mode1, METHOD calls DXDT and SETUP to generate the parameters needed by TRNSPT. METHOD calls TRNSPT to simulate radionuclide transport through the migration path for the problem simulation time. For time-dependent fluid velocities, METHOD executes the above sequence of commands for each time frame.

\section{2 .21 MXCLL(KBMAX)}

$\begin{array}{ll}\text { Called From } & \text { Calls } \\ \text { SETUP } & \text { INTG }\end{array}$

Subroutine MXCLL is called from SETUP when the source rate is distributed into the transport path based on a mixing-cell source region. MXCLL creates the source factor array used to update the fraction of the source of a given isotope to a specific grid block over a transport time step. MXCLL is structured according to the subcase numbering structure found in Appendix C. KBMAX is found in MXCLL and is the number of source grid blocks needed for the mixing-cell source. KBMAX is used in CHAIN to find the total number of grid blocks required for the source leg. 
3.2.22 PRP(D, B, V, VP, DL, N , T1, T2)

Called From

Calls

SETDIS

-...

Function PRP solves the integrals that are needed by SETDIS to create the discharge factor array. The discharge factor array is needed by the transport module to determine the discharge rates at the end of the migration path. The variables contained in the argument list are found in SETDIS and are used in the limits of integration.

\subsubsection{PTHLEN}

Called From
NEF
Calls

CHKPTH, TRACER

Subroutine PTHLEN is called from NEF and performs several tasks. First, if the flow network is solved, PHTLEN calculates the volumetric flow rates in each leg based on the pressures solved for in BSOLVE. At the user's option, PTHLEN prints pressures and flowrates of each leg in the network. Next, for the transport calculations, PTHLEN determines the pore fluid velocities for each leg in the migration path. PTHLEN calls CHKPTH to ensure that the pore velocities are consistent with the migration path (i.e., checks flow direction). For the averaged-leg DVM transport calculations, PTHLEN calculates the total path length from the source boundary to the discharge point. If the user opts, PTHLEN prints out path/velocity information after calling TRACER to find the nominal travel time, for steady-state velocities, or the maximum distance the fluid travels for time-dependent velocity fields.

\subsubsection{RATIO(DJ, DI, TIME, RAT)}

Called From
ET, SETDIS

RATIO is called from ET when the Taylor series approximation for finding the Bateman decay/production factors will require too much execution time to converge and is called from SETDIS to treat two-member production subchains. Ratio evaluates terms of the form

$$
\text { RATIO }=\frac{e^{-\lambda_{i} t}-e^{-\lambda_{j} t}}{\lambda_{j}-\lambda_{i}},
$$

where $\lambda_{i}$ is the half-life of isotope $i$ (DI), $\lambda_{j}$ the half-life of isotope $j(D J)$, and $t$ the time the decay/production factor is being found.

RATIO checks for extremes in half-life time products and for the smallness of differences in half-life values.

\section{2 .25 SETDIS}

Called From

SETUP
Calls

ET, PRP, RATIO 
Subroutine SETDIS is called from SETUP to create the discharge factor array. SETDIS finds this array by treating atoms that survive decay to discharge and by treating production subchains at discharge. To find atoms that survive decay to discharge, SETDIS calls ET to find the appropriate decay/production factors. To treat production subchains, an analytical treatment is used for two-member subchains. For this analytical treatment, SETDIS calls RATIO to find production/decay factors. For subchains having more than two members, SETDIS uses a velocity approximation scheme to treat subchains. To implement this approximation, integrals have to be evaluated and SETDIS calls PRP to evaluate the appropriate integrals. At the user's option, SETDIS prints out the discharge factor array to the appropriate output file (unit 6).

\section{2 .26 SETUP}

Called From METHOD
Calls

BRANCH, FACER, MXCLL, SETDIS TIMER

SETUP is called from METHOD to establish the source, transport, and discharge fractions for the DVM transport model. Specifically, SETUP performs the following functions:

1. Calls BRANCH to establish subchains for transport and find decay/production factors for the transport time step.

2. Distributes isotopic velocities normally with intervals of equal probability for each species in each leg.

3. Finds subchain velocities for transport by calling TIMER to find the mean time spent as each isotope for each subchain. This is only done when the number of isotopes input is greater than one.

4. Prints the velocity and decay/production information to the output file (unit 6) if the user has requested this in the input.

5. Determines the transport fractions by finding the pointer array (JV) which points to contributor blocks, the multiplier array (B) which is used to transport the material and the number array (N) which contains the maximum entries of the pointer array. If the user opts in the input, the multiplier array and the pointer array will be printed to the output file (unit 6).

6. Finds arithmetically averaged subchain velocities to be used in the mixing-cell source model and discharge model.

7. If the mixing-cell source model has been used, SETUP calls MXCLI to find the source factor array. MXCLL returns KBMAX, which is the number of source blocks needed if the mixing-cell source modelis used.

8. If the mixing-cell source model has been used, METHOD finds the total number of grid blocks needed based on KBMAX, and checks this total against the dimensions. If dimensions are exceeded but a 
statistical run is not being conducted, the program is stopped. If a statistical run is being conducted, KBMAX is reduced according to the dimensions and a warning is printed to the output file.

9. If the leg-to-leg transfer algorithm is to be used, SETUP calls FACER to find the transport fractions at the leg interfaces.

10. If the user has requested on input, the DVM time and space increments, the total number of grid blocks and the total number of catcher blocks, and the source type are printed to the hardcopy output file (unit 6 ).

11. Calls SETDIS to find the discharge fractions for the discharge model used in the DVM transport calculations.

\section{2 .27 SIFT(NSORT, TSCL)}

Called From

Calls

WORK

If the user requests, discharge rates for all isotopes in each chain are placed on the same time scale in WORK. WORK calls subroutine SIFT to sort the times into ascending order.

\subsubsection{SOURCE(IPASS, JTRIAL)}

Called From

NEF
Ca11s

BRANCH, CHAIN, DTUPDT, LECHMOD

Subroutine SOURCE implements the source model contained in NEFTRAN II by performing the following functions:

1. Calls BRANCH to update the waste inventory to the release time and prints release time inventory if the user has requested this on input. If the analytical transport model is being utilized, this is the only function performed by SOURCE.

2. Initializes the binary file (TAPE24, unit 24) of source rates that will be used by the DVM transport model.

3. If the time-dependent velocity capability of NEFTRAN II is being utilized, SOURCE intitalizes the relevant variables for interpolating the time-varying flowrate through the source regime.

4. Calls DTUPDT to find the source time step (if the user has not input one) and to age the inventory by the initial source time step.

5. If leaching is not included in the source model, depending on user input, SOURCE puts all the initial inventory into the leached-butundissolved inventory. If leaching is included in the source model, SOURCE calls LECHMOD to account for leaching between the leach time (inverse of the input leach rate) and the release time. 
6. Sets up the mixing-cell ratios to be used to determine source rates, if the source is being modeled as a mixing cell, and updates these ratios to the midpoint of the source time step for reporting.

7. If the source is a mixing cell and some leaching has occurred up to release time, SOURCE Initializes the mixing-cell concentrations based on the leached-but-undissolved inventory.

8. If the source is solubility-limited, then SOURCE updates the mixing-cell concentrations based on solubility-limits applied to the undissolved inventory. SOURCE subtracts the dissolved amount from the undissolved inventory.

9. Loops over source time steps. Subsequent steps are executed for each time increment.

10. Calls LECHMOD, if leach-limited source, to determine the amount leached over the time increment. This amount is added to the undissolved inventory.

11. If the source is a flow-through source and is only leach-limited, then SOURCE determines the source rate based on the amount leached during the time increment and the undissolved inventory. If the source is a mixing-cell source and is only leach-limited then SOURCE determines the source rate based on the amount leached, the mixing-cell ratios, and the flow rate through the source.

12. Finds total flow over the time increment by interpolating, if time-dependent flow rate through the source regime are being used.

13. Finds total amount dissolved based on solubility limits and the flow rate through the source and compares this amount to the current amount available in the undissolved inventory. The inventory dissolved is the minimum of these two amounts. Subtracts the dissolved amount from the undissolved inventory for the next time step.

14. Writes the source rates to the binary file (TAP24, unit 24) to be used by the DVM transport model and also to the appropriate output file (unit 25) if the user has opted for this on input.

15. Ages inventory by the source time step for the next time step.

\subsubsection{SRCIN (T, SR1, SR2, TS1, TS2, N1, N2, NTOTX, S24, IEOF)}

Called From
TRNSPT

SRCIN is called from TRNSPT to get the source rate over the transport time step and place it into the appropriate blocks in the first leg of the migration path (the source leg). SRCIN finds the total source per isotope for each transport time increment by interpolating the rates from the binary source rate file written by SOURCE (TAPE24, unit 24). This is 
necessary since the source and transport models are run independently and will not necessarily have the same time step. The variables listed in the argument list are used for the interpolation.

For the flow-through source model, SRCIN sums the atoms that are currently in the source blocks, adds the source rate found for the time step increment, and uniformly distributes the total amount into the source blocks. Also for each time increment, SRCIN sums the atoms that are in the source-leg catcher blocks, adds this sum to the total sum that is to be distributed uniformly, and sets atoms in the catcher blocks to zero. For the mixing-cell source, the source is distributed into grid blocks in the migration path according to the source factor array found in MXCLL.

\subsubsection{STOPPER(ISTOP)}

Called From

Calls

FLOWIN, GETRV

-...

STOPPER is called by FLOWIN and GETRV to check input data for consistency. STOPPER detects both fatal and non-fatal input errors and writes either stop messages or warning messages. NEFTRAN II, unlike its predecessors, does not use numbered stop statements, but a descriptive reason for program termination is provided on both the terminal screen and the hardcopy output file. ISTOP is a flag that indicates which parameter set is being checked. The only data set that currently is not checked by STOPPER is the increment-determination parameters.

\subsubsection{TIMER(KNT)}

Called From SETUP
Calls

TSPFAC

TIMER is called by SETUP to find the mean time spent during a time step as each isotope for each subchain (see Section 2.4.6). SETUP uses this information to find the subchain velocities needed for the DVM transport model. For a chain of five members or less, SRCIN does the calculation directly by calling TSPFAC. For chains of more than five members, TIMER estimates the time using an approximation scheme. If requested by the user in the input, TIMER prints the fractions of DT spent as each isotope.

\subsubsection{TPPRT(TM, TSET)}

Called From

BAND, METHOD, TRNSPT
Calls

-....-

TPPRT prints the discharge curves determined from the transport simulations to the hardcopy output file at a time frequency of IFREQ (input parameter), depending on a user input option. If the user also opts for the discharges rates only to be written to a file (unit 30), then TPPRT writes the discharge curves to temporary local files (unit 15 - 20, one file for each chain in the simulation), where the unit number is a 
function of the chain index. These local files are then used by wORK to write the final discharge rate file.

TPPRT is called from BAND every time step increment to print discharge curve points found using the analytical transport model. When called from TRNSPT every time increment, TPPRT prints discharge curve points found using the DVM transport model. In METHOD, if no discharge is detected during a simulation time for the analytical solution, TPPRT is called to print zero discharge points at the release time and the simulation time. TPPRT is also called from METHOD for the analytical transport solution, to append zeroes to the output files (unit 6 and/or unit 30), when TEND is less than the simulation time.

TPPRT converts the output from the transport models from atoms/yr to $\mathrm{Ci} / \mathrm{yr}$, where less than one atom/yr is considered exact zero. TPPRT also finds the time of the initial breakthrough, the total discharge and the peak discharge rate and time of occurrence for each isotope. In the argument list, TM is the time at which the discharge rate for each isotope is printed. TSET is a variable that is used to make the times at discharge is written consistent with the time source is assumed to be available for transport.

\subsubsection{TRACER(K, TT, PDIS)}

Called From

CHAIN, PTHLEN
Calls

-....-

TRACER is called from PTHLEN $(\mathrm{K}=0)$ to find the mean fluid travel time (TT) for steady-state velocity fields, and for time-dependent velocity fields, TRACER finds the maximum fluid travel distance (PDIS). When called from CHAIN, TRACER finds the mean isotopic travel time $(\mathrm{K}=$ isotope index $)$.

\subsubsection{TRNSPT(T, TSET, NTP, NPT, KALL)}
Called From
Calls
METHOD
SRCIN, TPPRT, XCHNG

TRNSPT is the DVM transport routine that performs the necessary multiplications to transport material over grid blocks for each time increment. TRNSPT accomplishes interior leg transport, leg-to-leg transport, and discharge by using multipliers developed in SETUP. TRNSPT loops over time from the time of source release to the upper time bound specified by the user. If time-dependent velocities are used, TRNSPT loops over time within each time interval. Within the time loop, TRNSPT performs the following functions:

1. Calls SCRCIN to inject the source into the appropriate grid blocks.

2. Calls TPPRT to write the discharge information to the appropriate output files. 
3. Calls XCHNG to simulate diffusion to and from the rock matrix, if the matrix diffusion model is being implemented.

4. Sums the atoms that are currently in the catcher blocks for each leg in the migration path, puts the sum into the first grid block of that leg, and sets the atoms contained in the catcher blocks to zero.

After transport is complete, TRNSPT rewinds the source rate file (unit 24 ) and prints the atom count summary to the output file (unit 6) if the user has opted for this to be printed in the input options.

3.2.35 TSPFAC(DT, D, B, LT, TDP)

Called From Calls

TIMER

-...-

Function TSPFAC is called from TIMER to estimate the mean time a particle spends as each isotope over a time step for chains of five members or less. TSPFAC uses a Taylor series to calculate the Bateman coefficients that are needed to estimate this mean time.

3.2.36 WORK(IPASS, JTRIAL)

$\begin{array}{ll}\text { Called From } & \text { Calls } \\ \text { NEF } & \text { SIFT }\end{array}$

WORK is called from NEF after the transport simulation has been completed and performs several different functions. Initially, WORK condenses the temporary output arrays to account for non-transported decay chains, computes the weighted integrated discharges for each isotope, and prints an optional discharge summary for all of the isotopes to the hardcopy output file (unit 6 ) if the user requests this on input.

The remaining function of WORK is to write discharge rates to the discharge rate file (unit 30 ) based on the temporary files (unit 15 - 19, one file for each chain being transported) written by TPPRT, if the user has requested this output file to be written in the input options. The user may also request on input for all the isotope discharge rates to be written to the discharge file on the same time scale. Different time scales may arise because different DVM transport time steps are allowed for each different radioactive decay chain. To put all of the rates on the same time scale, WORK develops a general time scale based on the first minimum breakthrough time for all the isotopes and the simulation time. WORK appends the peak times and the simulation time to this general time scale and calls SIFT to sort the times into ascending order. WORK consolidates the sequence by accounting for ties and eliminates times exceeding the problem simulation time. WORK linearly interpolates times and the logarithm of the discharge rates from the temporary discharge files to find the discharge rates at the general time scale times. 


\subsubsection{XCHNG}

Called From

Cal1s

TRNSPT

XCHNG is called from the DVM transport model routine, TRSNPT, to implement the matrix diffusion model contained in NEFTRAN II, if the user has designated a leg as a dual porosity leg. Subroutine XCHNG is called at the end of each time step by TRNSPT to update the concentrations because of exchange with the matrix (i.e., immobile phase). The exchange is accomplished with a simple, linear mass-transfer equation and the concentration gradient is not allowed to change sign over a time step. XCHNG updates the concentration in the matrix caused by decay and production for the next time step.

\subsection{Parameter Statements}

The include file (SIZES.INC) required by NEFTRAN II stores the set of PARAMETER statements that define the dimensions of the arrays used in the code. If the user needs to change the dimensions of the arrays, only the PARAMETER statements have to be changed in the include file. These parameter statements are listed below along with definitions of each dimension parameter used by NEFTRAN II.

PARAMETER (MXLEG $=25$, MXJCT $=25, M X L J=5, N U L C D=5$ )

PARAMETER (MXMEM $=10$, MXCHNS $=6$, MXPTH $=10$, MXNVI $=7$ )

PARAMETER ( $M X G R D=1000, N D D F=2500, N D S F=2500$ )

PARAMETER (NDJPN=2000, NDBF $=6000$, MXELEM=30)

PARAMETER (MXTDV $=20$, MXQSC $=100$ )

PARAMETER (MXISO $=$ MXMEM $*$ MXCHNS)

PARAMETER (MXSUB $=($ MXMEM $*($ MXMEM +1$)) / 2)$

PARAMETER (MXTSP $=($ MXMEM* $($ MXMEM +1$) *($ MXMEM +2$)) / 6-$ MXMEM)

PARAMETER (MBDIM $=$ MXSUB - MXMEM +1 )

PARAMETER (IBFDM $=($ MBDIM -1$) *($ MXPTH -1$) * 2)$

MXLEG - The maximum number of legs for the flow network

MXJCT - The maximum number of junctions for the flow network

MXLJ - The maximum number of legs entering or exiting any junction

NULCD - The maximum absolute difference between connected junction numbers (Does not apply to junctions with known pressures)

MXMEM - The maximum number of isotopes per decay chain

MXCHNS - The maximum number of decay chains

MXPTH - The maximum number of legs in the migration path

MXNVI - The maximum number of intervals for velocity distribution

MXGRD - The maximum number of grid blocks

NDDF - The maximum dimension of the discharge-factor array

NDSF - The maximum dimension of the source-distribution array

NDJPN - The maximum dimension of the leg-transfer pointer array

NDBF - The maximum dimension of the leg-transfer fractions array

MXELEM - The maximum number of elements

MXTDV - The maximum number of time frames allowed to simulate timedependent velocity fields

MXQSC - The maximum number of times allowed to simulate timedependent flow through the source regime 
The remaining PARAMETERS are functions of the above list and are defined as :

MXISO - The maximum total number of isotopes

MXSUB - The maximum number of production subchains per chain

MXTSP - The maximum dimension of the average time spent array

MBDIM - The maximum number of subchains of length $>1$

IBFDM - The maximum row dimension of the adjustment arrays

\subsection{Common Blocks}

A11 common blocks in NEFTRAN II are labeled and contain most of the arrays that are used by the code. In addition, there are a few arrays specific to certain subroutines. The following lists each common block statement along with a brief description of the labeled common block, and the arrays and parameters contained in the block. This listing is in alphabetical order according to common block label name.

\section{COMMON / BFIX / MBFX(MBDIM), IBFX(IBFDM, 2), ABFX(IBFDM, 2)}

This block is only used if the leg-to-leg transfer algorithm is invoked. In that case, negative velocities can cause double counting of some particles. This is corrected by applying the correction fractions stored here. All fractions are calculated in subroutine ADJB and are applied in subroutine TRNSPT. The name BFIX arises from the notion that the atoms/ block calculated by the B-ARRAY are being fixed.

MBFX - MBFX(I) points to adjustment-fraction block numbers in the IBFX array for production subchain I. It is set in FACER and used in TRNSPT. In particular, contributor and receiver block indices that require correction for production subchain. $I$ are stored from $\operatorname{IBFX}(\operatorname{MBFX}(I)+1, *)$ to $\operatorname{IBFX}(\operatorname{MBFX}(I+1), *)$.

IBFX - Column 1 is a contributor block and 2 is a receiver block index that require correction for each production subchain. It is found in ADJB, printed in FACER, and used in TRNSPT.

$A B F X$ - As part of calculating transport, TRNSPT applies the correction fraction in column 1 of $A B F X$ to the contents of block $\operatorname{IBFX}(, 1)$ and adds the product to block $\operatorname{ABS}(\operatorname{IBFX}(, 2))$. If $\operatorname{IBFX}(, 2)>0$, TRNSPT applies column 2 to the contents of $\operatorname{IBFX}(, 1)$ and adds the product to block $\operatorname{IBFX}(, 2)+1$. $A B F X$ is found in $A D J B$ and is printed in FACER.

COMMON/ DENSER / PEAK(MXISO), TIMPK(MXISO), TOTD(MXISO),

1 TIMBRK(MXISO), DWT(MXISO), WDIS (MXISO), WDISUM, NONZ

The name DENSER arises from the original intent of these arrays, to be used in condensing discharge rates in NEFTRAN (for a description see Longsine and others, 1987). In NEFTRAN II, condensing discharge rates has been replaced by the capability to place discharge rates from different chains on the same time gridding. 
PEAK - Peak discharge rates for each isotope ( $\mathrm{Ci} / \mathrm{y})$, initialized in NEF, found in TPPRT, used and printed in WORK.

TIMPK - Time of occurrence of the peak discharge rate (y), initialized in NEF, found in TPPRT, used and printed in WORK.

TOTD - Total discharge for each isotope (Ci), initialized in NEF, found in TPPRT, used and printed in WORK.

TIMBRK - Time of first nonzero discharge rate for each isotope (y), initialized in NEF, found in TPPRT, used in WORK.

DWT - Total-discharge weighting factor for each isotope (/Ci), read in FLOWIN, printed in INPRIN, and applied in WORK.

WDIS - Normalized total-discharge for each isotope, found and printed in WORK.

WDISUM - Sum over all normalized discharges, found and printed in WORR.

NONZ - Number of isotopes having nonzero discharge, initialized in NEF, found in TPPRT, used in WORK.

COMMON/ FLOW / C(MXJCT), AW(MXJCT, 2*NULCD+1),

$1 \mathrm{JL}$ (MXJCT,MXLJ), LJ (MXLEG, 2), JEQ(MXJCT), IB (MXJCT),

2 BCJ (MXJCT), NW, NL, NU, NB

This COMMON block contains information to set up and solve the flow network. The solution is cast as the matrix equation $A x=b$. In the notation below, matrix $A$ is called $A W$ and both the constant (b) and solution $(x)$ vectors are stored in array $C$.

$\mathrm{C}$ - In COEFF, $\mathrm{C}$ is the constant vector containing the appropriate transmissibility-pressure products. In BSOLVE, $\mathrm{C}$ is converted to the calculated pressures, which are substituted into the pressure array, $P$ of COMMON JUNCT, by NEF.

AW - Banded matrix of transmissibilities, developed in COEFF and used in BSOLVE. Note that the bands are stored in rectangular form with each co-diagonal as a column.

JL - Legs connected to each junction, used to set off-diagonal entries in array AW by COEFF. Legs whose flow-direction is into the junction are labeled negatively and outflow legs are positive.

IJ - Junctions terminating each $1 \mathrm{eg}$, read in FLOWIN, printed in INPRIN, used in COEFF, PTHLEN, and CHKPTH to develop the JL array and to reference junction properties.

JEQ - For each junction, stores its associated row index in the matrix equation (if the junction has unknown pressure) or zero (if the junction has known pressure) in COEFF. In NEFMAIN, JEQ is used to replace the solved pressures of vector $C$ into vector $P$ of COMMON JUNCT. Note also that this array is temporarily used in FLOWIN, STOPPER, and INPRIN to store and print junction pressure-boundary flags.

IB - Junction numbers having user-specified pressures, developed in FLOWIN and used in COEFF.

BCJ - Known pressures for the junctions stored in array $I B\left(1 \mathrm{~b} / \mathrm{ft}^{2}\right)$, developed in FLOWIN and used in COEFF.

NW - Number of rows of matrix $A W$ and vector $C$, determined in COEFF and used in BSOLVE.

NL - Number of lower codiagonals of matrix AW, determined in COEFF and used in BSOLVE. 
$\mathrm{NB}$ - Number of junctions with user-specified pressures, developed in FLOWIN and used in COEFF and NEF.

COMMON / INAME / ISONAM(MXMEM), NAMALL(MXISO)

CHARACTER *6 ISONAM, NAMALL

Isotope names are stored in the arrays of this common block. Each occurrence of this block is accompanied by the CHARACTER declaration shown. As evident from the CHARACTER declaration, isotope names can be no more than 6 characters.

ISONAM - Isotope names for the chain being transported, set in CHAIN from the NAMALL array and used for printing in subroutines BAND, SETUP, and TRNSPT.

NAMALL - Isotope names for all isotopes, read in FLOWIN, used in CHAIN, and used for printing in INPRIN, SOURCE, and WORK.

COMMON/ ISOTOP / CUROUT(MXMEM), LAMBDA(MXMEM), THALF (MXMEM),

1 VELISO (MXPTH, MXMEM), CI2ATM (MXMEM), IFR (MXMEM), BRFR (MXMEM),

2 NOISO, HLFALL(MXISO), LMTX(MXISO), CURALL(MXISO),

3 IPRALL(MXISO, 2), FPRALL(MXISO, 2), TRAVT(MXISO), NI(MXCHNS),

4 INCHN(MXCHNS), NCHNS, NTOT, MFNDX, ICH

This common contains information for all isotopes (dimensioned by MXISO) in all chains (dimensioned by MXCHNS) and has arrays designated for storing data for the current chain being transported (dimensioned by MXMEM). On input, all decay chain data are read, but following the printing of these data in INPRIN, FLOWIN compacts all arrays dimensioned by MXISO and MXCHNS to include only those chains marked for inclusion in source calculations. The descriptions that follow are pertinent for both data input (through printing) and compacted arrays (for subsequent use). Each occurrence of this common block is accompanied by type statement that declares variable LAMBDA as DOUBLE PRECISION.

CUROUT - Discharge rates for each isotope of the chain being transported at the current time $(\mathrm{Ci} / \mathrm{y})$, initialized to zero in METHOD, calculated for each time step in BAND and TRNSPT in atoms $/ y$, and converted and printed in TPPRT.

LAMBDA - Decay constants for each isotope of the chain being transported $(1 / y)$, set to $\ln (2) /$ THALF in CHAIN, used in BRANCH, DTUPDT, SETDIS, MXCLL, TIMER, and BAND. Several other routines use the values in LAMBDA, but the values are passed to these routines via the calling sequence, usually in array $D$ (which is also declared as DOUBLE PRECISION).

THALF - Half-1ives for each isotope of the chain being transported (y), set from the HLFALL array in subroutine CHAIN and used in CHAIN and DXDT.

VELISO - Retarded velocities in each migration path leg for each isotope of the chain being transported $(\mathrm{ft} / \mathrm{y})$, found and stored in TRACER for steady-state flow runs. For time-dependent flow runs, VELISO is over-written for each time interval in TRACER. For each time interval, including a steady-state frame, TRACER 
provides VELISO to DXDT to find apace/time gridding for the time frame. In METHOD, elther the steady-state VELISO is used or VELISO is re-computed for each time frame. In either case, VELISO is then used in BAND and SETUP.

CI2ATM - Factors used to convert Curles to atoms for each 1sotope of the chain being transported (atoms/C1), calculated in CHAIN as $1.6834 \times 10^{18} \times$ THALF and used in BAND and TPPRT. This conversion also occurs in the SOURCE routine, although CI2ATM is not explicitly used there.

IFR - A daughter indicator for each isotope of the chain being transported, developed in DTUPDT and over-written then redeveloped in CHAIN for use in BRANCH, SETDIS, MXCLL, and TIMER. For each isotope I, IFR(I) - 0 unless isotope I splits its decay to two daughters, in which case IFR(I) is the index of the first daughter encountered.

BRFR - A branching fraction for each lsotope of the chain being transported, developed in DTUPDT and over-written then redeveloped in CHAIN for use in BRANCH, SETDIS, MXCLL, and TIMER. $\operatorname{BRFR}(I)=0$, unless IFR(I) $\neq 0$, in which case BRFR(I) is the branching fraction from parent I to daughter IFR(I). Note that the fractions in BRFR are subtracted from one and sent via calling sequence to several supporting routines, usually in array BFR.

NOISO - Number of isotopes in the chain being transported, used as a local variable and over-written in STOP.PER, SOURCE, and DTUPDT, and set for decay chain transport in CHAIN from the NI array. The value set in CHAIN is used in DXDT, BRANCH, SETUP, SETDIS, FACER, MXCLL, TIMER, METHOD, BAND, TPPRT, TRNSPT, SRCIN, and XCHNG.

HLFALL - Half-1ives for all isotopes (y), read in FLOWIN, checked in STOPPER, printed in INPRIN, and used in SOURCE, CHAIN, and DTUPDT.

LMTX - Element index for all isotopes, read in FLOWIN, checked in STOPPER, printed in INPRIN, and used in SOURCE and CHAIN to assign solubilities and retardations, respectively.

CURALL - Initial inventories for all isotopes (Ci), read in FLOWIN, checked in STOPPER, printed in INPRIN, and used in SOURCE.

IPRALL - Parent identifiers for all isotopes, read in FLOWIN, checked in STOPPER, printed in INPRIN, and used in CHAIN, BRANCH, and DTUPDT .

FPRALL - Branching fractions for all isotopes, read in FLOWIN, checked in STOPPER, printed in INPRIN, and used in CHAIN and DTUPDT.

TRAVT - Mean travel time for all isotopes ( $y$ ), set in CHAIN by calling TRACER and used in DTUPDT and DXDT. There are two cases where TRAVT is set to an arbitrarily large number for an isotope by CHAIN - (1) if the isotope is in a decay chain that is included in source calculations but not transported and (2) if, for a time-dependent velocity run, the isotope cannot reach the discharge point in the total time simulated.

NI - Number of isotopes per chain, read in FLOWIN, checked in STOPPER, and used in SOURCE, CHAIN, DTUPDT, TRNSPT, WORK, and INPRIN. 
INCHN - Inclusion flag for each chain, read in FLOWIN, checked in STOPPER, printed in INPRIN, and used in NEF, SOURCE, CHAIN, TRNSPT, and WORK. On input, each entry can be 0,1 , or 2 . However, as stated above, all information for chains not included (2) is discarded, and the only values of INCHN then possible are 0 (source and transport) or 1 (source only).

NCHNS - Number of chains for source calculations, calculated in FLOWIN, checked in STOPPER, and used in NEF, SOURCE, DTUPDT, and WORK.

NTOT - Total number of isotopes for source calculations, calculated in FLOWIN and used in SOURCE, DTUPDT, and WORK.

MFNDX - Cumulative number of isotopes to the end of the previous chain, calculated and over-written in SOURCE, DTUPDT, and WORK and determined for transport of each chain in CHAIN. The latter value is then used to reference arrays in BRANCH, DXDT, and TPPRT.

ICH - Index of the chain being transported, set and over-written in SOURCE, DTUPDT, and WORK and set for transport of each chain in NEFMAIN. The latter value is then used to reference arrays and disc file unit numbers in CHAIN, METHOD, TPPRT, and TRNSPT.

\section{COMMON / JUNCT / P(MXJCT), EL(MXJCT)}

This block contains all junction properties, with the exception of the boundary conditions found in COMMON FLOW (IB, BCJ, and NB).

$P$ - Pressures at all junctions $\left(1 \mathrm{~b} / \mathrm{ft}^{2}\right)$. In FLOWIN, $P$ is used to temporarily store the user-specified boundary pressures (in psi units) and is zeroed out after being used to print the input pressures in INPRIN. In COEFF, the boundary pressures (converted from psi to $1 \mathrm{~b} / \mathrm{ft}^{2}$ in FLOWIN and stored in array BCJ, see COMMON FLOW) are placed into the appropriate locations in the $P$ array. In NEFMAIN, the calculated pressures (see array C, COMMON FLOW) are slotted into the remaining locations. Subroutine PTHLEN uses the pressures in $P$ to calculate flow rates for each $\mathrm{leg}$ and optionally prints $P$ in input units, psi.

EL - Elevations for each junction ( $f t$ ), read in FLOWIN, printed in INPRIN, and used in COEFF and PTHLEN,

\section{COMMON/ LEGS / AREA(MXLEG), BC(MXLEG), COND(MXLEG), Q(MXLEG), 1 FDENS (MXLEG), PATH (MXLEG), TH(MXLEG)}

The leg properties stored here relate to the flow network, so each array is dimensioned to the maximum number of network legs, as opposed to leg properties specific to the migration path which are stored in COMMON MISC, discussed below. If the flow network is not solved, the only array used from this block is the leg-lengths array, PATH.

AREA - Cross-sectional area perpendicular to flow for each leg ( $f t^{2}$ ), read in FLOWIN, checked in STOPPER, printed in INPRIN, and used in COEFF and PTHLEN. 
BC - Relative brine concentration for each leg (dimensionless between 0 and 1 ), read in FLOWIN, checked in STOPPER, printed in INPRIN, and used in COEFF.

COND - Hydraulic conductivity for each leg ( $\mathrm{ft} / \mathrm{y}$ ), read in FLOWIN in units ft/day, printed in INPRIN, and then converted in FLOWIN to $\mathrm{ft} / \mathrm{y}$ for use in COEFF.

Q - Volumetric fluid flow rate through each leg $\left(\mathrm{ft}^{3} / \mathrm{y}\right)$, calculated in PTHLEN and checked in CHKPTH. Note, if velocities are input directly, $Q$ is equated to velocity in each leg prior to the call to CHKPTH for checking flow directions.

FDENS - Fluid density of each leg ( $\left.\mathrm{lb} / \mathrm{ft}^{3}\right)$, calculated in COEFF and used in PTHLEN.

PATH - Length of each leg ( $f t$ ), read in FLOWIN, checked in STOPPER, printed in INPRIN, and used in COEFF, PTHLEN, TRACER, CHAIN, SETUP, FACER, and DXDT.

TH - Transmissibility for each leg (mixed units), calculated and used in COEFF and used again in PTHLEN.

COMMON/ MISC / ALPHA(MXPTH), PHI(MXPTH), PORE(MXPTH), DXDTPA(9), 1 DTCHN(MXCHNS), DT, Y, MPATH(MXPTH), LCH, MXC, LTOLX, IXCHG,

2 NELM, NLEG, NJCT, NPATH

As the name MISCellaneous suggests, this is somewhat of a catchall common block. Migration path properties and the time step are the most often used variables listed here.

ALPHA - Dispersivities for each leg in the migration path ( $f t$ ), read in FLOWIN, checked in STOPPER, printed in INPRIN, and used in DXDT, BAND, and SETUP. For the analytical solution transport model and for path-averaged-DVM transport using leg-averaged velocities, only one value of dispersivity is used. It is taken to be the first value of the array, ALPHA(1).

PHI - Mobile phase porosity for each leg in the migration path, read in FLOWIN, checked in STOPPER, printed in INPRIN, and used in PTHLEN to convert steady-state Darcy flux to pore velocity and in XCHNG to compute mobile phase concentrations.

PORE - Steady-state pore velocity for each leg in the migration path $(\mathrm{ft} / \mathrm{y})$, read in FLOWIN and, if the flow network is solved, over-written in PTHLEN. PORE is checked in STOPPER, printed in INPRIN, and used in TRACER and CHAIN.

DXDTPA - Increment-determination parameters for the DVM transport model, read or defaulted in FLOWIN and used in DXDT.

DTCHN - DTCHN(I) is the user-specified time step for the transport of decay chain $I$, read in FLOWIN, printed in INPRIN, and used in CHAIN. If input as zero, DXDT determines a time step for that chain. Note that for time-dependent velocity runs, the userspecified value may be modified so that there is an integer number of time steps for each time interval.

DT - Current time step for source or transport (y). If provided by the user, DT for source is equated to DTSRC in DTUPDT. DT for the DVM transport model is equated to DTCHN(I), for each chain $I$, in CHAIN and DT is calculated in METHOD if the analytical 
solution transport model is chosen. Since the time step for transport can change for the time intervals specified by timedependent velocity data, accepted values of DT for each time frame (determined in DXDT) are stored in the TDDT array of COMMON VELFLD, for retrieval by routine METHOD. The source DT is used by SOURCE, BRANCH, and DTUPDT. The transport DT is used by CHAIN, DXDT, SETUP, BRANCH, TIMER, MXCLL, SETDIS, METHOD, BAND, TPPRT, TRNSPT, SRCIN, and XCHNG. There is one other use for DT. DT is temporarily set to release time (TRLSE, see COMMON OUTVC) by subroutine SOURCE prior to calling BRANCH, in order to update the inventories to TRLSE.

$Y$ - Distance from the leading edge of the source regime to the discharge boundary ( $f t$ ), computed by adding leg lengths in PTHLEN. $Y$ is reported in PTHLEN and used in TRACER, CHAIN, DXDT, SETUP, METHOD, and BAND.

MPATH - Migration path leg indices, read in FLOWIN, checked in STOPPER, printed in INPRIN, and used in PTHLEN, CHKPTH, TRACER, CHAIN, DXDT, SETUP, and FACER.

LCH - Source model flag: (0) leaching only, (1) solubilities only, or (2) both are considered in finding source rates. Set in STOPPER to option 19 and used in FLOWIN and DTUPDT.

MXC - Source model flag: (0) fluid flow-through, (1) fluid dilution as modeled by a mixing cell, or (2) let code choose between 0 and 1 to distribute source rates into transport path. Set in STOPPER to option 20 and used in FLOWIN.

LTOLX - A DVM transport flag; (0) leg-averaged velocities or (1) the leg-to-leg transfer algorithm will be used to transport radionuclides. LTOLX is set in FLOWIN to option 22, but may be over-written if option 22 is zero and matrix diffusion is to be modeled or time-dependent flow fields are input. LTOLX is used in CHAIN, DXDT, SETUP, METHOD, and TRNSPT.

IXCHG - A DVM transport flag; (0) no matrix diffusion or (1) yes matrix diffusion will be included for transport. IXCHG is set in FLOWIN after reading the migration path properties, in particular, the leg-dependent diffusion flags. IXCHG is used in STOPPER and TRNSPT.

NLEG - Number of legs in the flow network, read in FLOWIN, checked in STOPPER, printed in INPRIN, and used in COEFF and PTHLEN.

NJCT - Number of junctions in the flow network, read in FLOWIN, checked in STOPPER, printed in INPRIN, and used in NEF, COEFF, and PTHLEN.

NPATH - Number of legs in the migration path, read in FLOWIN, checked in STOPPER, printed in INPRIN, and used in PTHLEN, CHKPTH, TRACER, CHAIN, DXDT, SETUP, SETDIS, METHOD, and XCHNG.

COMMON/ OPTION / IOPT(38)

The intent of individual options is described in Section 4.3.1, so only the definition and use of the entire array is mentioned here.

IOPT - Options, read in FLOWIN, checked in STOPPER, printed in INPRIN, and used in NEFMAIN, FLOWIN, STOPPER, INPRIN, COEFF, PTHLEN, TRACER, SOURCE, CHAIN, DTUPDT, DXDT, SETUP, TIMER, FACER, 
MXCLL, SETDIS, METHOD, BAND, GIT, TPPRT, TRNSPT, WORK, and GETRV.

COMMON / OUTVC / TUB, TRLSE, TLCH, SUMIN(MXMEM), SUMOUT(MXMEM), 1 ICOUNT, IFREQ, QDAREA, QDIS

This common block (OUTVC) contains some output controls and summary information.

TUB - Problem simulation time (y), read in FLOWIN, printed in INPRIN, and used in NEFMAIN, PTHLEN, TRACER, SOURCE, CHAIN, DTUPDT, METHOD, TPPRT, and WORK.

TRLSE - Time of initial radionuclide-fluid contact (y), read in FLOWIN, checked in STOPPER, printed in INPRIN, and used in PTHLEN, TRACER, SOURCE, CHAIN, DTUPDT, DXDT, METHOD, TPPRT, TRNSPT, and SRCIN.

TLCH - Time of onset of leaching (y), read in FLOWIN, checked in STOPPER, printed in INPRIN, and used in SOURCE and LECHMOD.

SUMIN - Total amount input as source for DVM transport for each isotope (atoms), zeroed-out in METHOD, accumulated in SRCIN, and reported in TRNSPT.

SUMOUT - Total amount discharged from DVM transport for each isotope (atoms), zeroed-out in METHOD, accumulated and reported in TRNSPT.

ICOUNT - Time step counter for frequency of printing discharge rates to hardcopy output file (unit 6), initialized in METHOD (for DVM) and BAND (for analytic solution) and used and reset in TPPRT.

IFREQ - Discharge rates are printed to the hardcopy output file (unit 6) every IFREQ time steps. IFREQ is set to the absolute value of option 10 in FLOWIN and is used in METHOD, BAND, AND TPPRT.

QDAREA - Product of cross-sectional area perpendicular to flow and porosity, at the discharge point $\left(f t^{2}\right)$, read in FLOWIN, checked in STOPPER, printed in INPRIN, and used to calculate QDIS, below, in PTHLEN and METHOD.

QDIS - Flowrate at discharge $\left(\mathrm{ft}^{3} / \mathrm{y}\right)$, either set to the calculated flowrate of the last leg in the migration path by PTHLEN or, if the network is not solved, is set to the product of QDAREA and the pore velocity of the last leg in the migration path by PTHLEN (steady-state runs) or METHOD (time-dependent flow runs). QDIS is used in TPPRT to convert radionuclide discharge rates to concentrations.

COMMON/ QSOURCE / TSRCE (MXQSC), QSRCE (MXQSC), NQSC

This common contains information to simulate a time-varying flowrate through the source regime. There can be fixed flow (in which case none of the data here is required) or time-varying flow for steady-state runs. However, runs using time-dependent velocities automatically generate time-varying source flowrates, if not input explicitly designated by the user. 
TSRCE - TSRCE(I) is the time to which flowrate QSRCE(I) applies (y), and is either read in FLOWIN from an external file (see Section 4.3.1) or set in FLOWIN to the time frames specified for the time-dependent velocities given in TDTM of COMMON VELFLD. TSRCE is checked in STOPPER, printed in INPRIN, and used in SOURCE and DTUPDT.

QSRCE - QSRCE(I) is the flowrate through the source regime which applies to time TSRCE(I) $\left(\mathrm{ft}^{3} / \mathrm{y}\right)$, and is either read in FLOWIN from an external file or calculated in FLOWIN as the product of the time-dependent pore velocities given in the first column of TDVEL of COMMON VELFLD and QSAREA of COMMON SOLIMT. QSRCE is checked in STOPPER, printed in INPRIN, and used in SOURCE and DTUPDT .

NQSC - Number of times specified for simulating time-dependent flow through the source regime, set in FLOWIN (upon encountering an end-of-file marker or set to MFL2 of COMMON VELFLD if the timedependent velocities were used) and used in INPRIN, SOURCE, and DTUPDT .

COMMON / RETARD / AKI (MXPTH,MXELEM), AKIM(MXPTH,MXELEM), 1 RKI (MXPTH, MXMEM), RKIM(MXPTH, MXMEM)

The retardation factors for both the mobile and immobile phases are stored here by element and leg for all elements and by isotope and leg for the isotopes of the chain being transported.

AKI - Retardation factors in the mobile phase for each migration leg and each element, read in FLOWIN, checked in STOPPER, printed in INPRIN, and used in CHAIN.

AKIM - Retardation factors in the immobile phase for each migration leg and each element, read in FLOWIN, checked in STOPPER, printed in INPRIN, and used in CHAIN.

RKI - Retardation factors in the mobile phase for each migration leg and each isotope in the chain being transported, set in CHAIN and used in CHAIN, TRACER, METHOD, and XCHNG.

RKIM - Retardation factors in the immobile phase for each migration leg and each isotope in the chain being transported, set in CHAIN and used in XCHNG.

COMMON / SOLIMT / CSALL(MXELEM), AMALL(MXISO), DTPAR(7), ACSSFR,

1 LLONLY, IDSRC, VOL, QSAREA, QSC, LMODEL, RLCH, SAL, DTSRC

Most of the information required for source calculations is included in this common block.

CSALL - Solubility limit for each element (g-element/g-fluid), read in FLOWIN, checked in STOPPER, printed in INPRIN, and used in SOURCE.

AMALL - Atomic mass for each isotope, read in FLOWIN, checked in STOPPER, printed in INPRIN, and used in SOURCE. 
DTPAR - Source time-step determination parameters, read or defaulted in FLOWIN and used in DTUPDT

ACSSFR - Fraction of the inventories input in CURALL of COMMON ISOTOP to be used for source calculations, read in FLOWIN, checked in STOPPER, printed in INPRIN, and used in SOURCE and BAND.

LLONLY - Source control parameter which is assigned one of the first six values of IDSRC (below) following the mixing cell feasibility check, set in DTUPDT and used in SOURCE, SETUP, and SRCIN.

IDSRC - Source control parameter for leach/solubility and mixing cell, set to $3(\mathrm{MXC})+$ LCH (see COMMON MISC) by FLOWIN and used in DTUPDT .

VOL - Pore volume of the source regime for mixing cell calculations $\left(\mathrm{ft}^{3}\right)$, read in FLOWIN, checked in STOPPER, printed in INPRIN, and used in SOURCE and DTUPDT.

QSAREA - Product of cross-sectional area perpendicular to flow and porosity of the source regime $\left(f t^{2}\right)$, read in FLOWIN, checked in STOPPER, printed in INPRIN, and used to calculate QSC, below, in PTHLEN and to calculate QSRCE of COMMON QSOURCE in FLOWIN.

QSC - Fluid flow rate through the source regime $\left(\mathrm{ft}^{3} / \mathrm{y}\right)$. For steadystate source-flow runs, QSC is set in PTHLEN to the calculated flow rate of the first leg in the migration path (if the flow network is solved) or to the product of QSAREA and the pore velocity of the first leg in the migration path (if the flow network is not solved). For time-dependent runs, QSC is set to the flow rate in QSRCE of COMMON QSOURCE that corresponds to the appropriate time interval. QSC is used in SOURCE and DTUPDT.

LMODEL - A leach model flag; (0) constant leach rate or (1) exponential leach rate is used in finding source rates. Set in STOPPER to option 21 and used in LECHMOD.

RLCH - Leach rate for the specified leach model $(1 / y)$, read in FLOWIN, checked in STOPPER, printed in INPRIN, and used in DTUPDT, LECHMOD, and BAND.

SAL - Fluid density in the source regime $\left(1 \mathrm{~b} / \mathrm{ft}^{3}\right)$, read in FLOWIN, checked in STOPPER, and printed in INPRIN. If the flow network is solved, SAL is replaced in PTHLEN by the fluid density calculated for the first leg in the migration path. SAL is used in SOURCE.

DTSRC - User-specified time step for source calculations (y), read in FLOWIN, printed in INPRIN, and checked and used in DTUPDT. If specified as zero, DTUPDT determines the time step.

COMMON / VELFLD / TDTM(MXTDV), TDVEL(MXTDV,MXPTH),

1 TDSAT (MXTDV, MXPTH), TDDT(MXTDV), MFL1, MFL2

Data to simulate time-dependent velocity fields is included in this common block.

TDTM - TDTM(I) is the time to which the velocities TDVEL(I,MXPTH) apply (y), read in FLOWIN from an external file, checked in STOPPER, and printed in INPRIN. TDTM is used in FLOWIN to set TSRCE of COMMON QSOURCE (if TSRCE is not specified). TDTM is 
also used in TRACER, CHAIN, and METHOD. For steady-state runs, TDTM(1) is set to TUB of COMMON OUTVC by FLOWIN and only the first entry is referenced.

TDVEL - TDVEL $(I, J)$ is the pore fluid velocity for the $J^{\text {th }}$ leg in the migration path that applies to time $\operatorname{TDTM}(\mathrm{I})$ ( $\mathrm{ft} / \mathrm{y}$ ), read in FLOWIN from an external file, checked in STOPPER, and printed in INPRIN. TDVEL $(I, 1)$ is used in FLOWIN with QSAREA of COMMON SOLIMT to calculate QSRCE of COMMON QSOURCE, if QSRCE is not specified. TDVEL is also used in PTHLEN, TRACER, CHAIN, and METHOD. For steady-state runs, $\operatorname{TDVEL}(1, \mathrm{~J})$ is set to PORE(J) of COMMON MISC by PTHLEN and only the first time entry is referenced.

TDSAT - TDSAT(I,J) is the saturation for the $J^{\text {th }}$ leg in the migration path that applies to time TDTM(I), read in FLOWIN from an external file, checked in STOPPER, and printed in INPRIN. TDSAT is used in TRACER and METHOD. For steady-state runs, $\operatorname{TDSAT}(1, \mathrm{~J})$ is set to one for all migration legs by PTHLEN and only the first time entry is referenced.

TDDT - TDDT(I) is the time step, for the chain being transported for time frame $I(y)$, set in CHAIN following the call to DXDT, and retrieved by METHOD. For steady-state runs, TDDT(1) is set to DT of COMMON MISC by CHAIN following a call to DXDT and only the first entry is referenced.

MFL1 - Smallest index in TDTM such that time TDTM(MFL1) is at least as large as release time, TRLSE of COMMON OUTVC. MFL1 is found in STOPPER, but is set to one by FLOWIN for steady-state runs. MFL1 is used in PTHLEN, TRACER, CHAIN, and METHOD.

MFL2 - Smallest index in TDTM such that time TDTM(MFL2) is at least as large as simulation time, TUB of COMMON OUTVC. MFL2 is found in STOPPER, but is set to one by FLOWIN for steady-state runs. MFL2 is used in PTHLEN, TRACER, CHAIN, and METHOD.

COMMON / XFER / NX(MXPTH), NC(MXPTH), DX(MXPTH), TMN(MXTSP),

$1 \mathrm{BF}(\mathrm{NDBF}), \mathrm{JPSC}(\mathrm{MXSUB}+1)$, JPN(NDJPN , 3), IMB (MXPTH),

$2 \operatorname{PHIM}(\mathrm{MXPTH}), \mathrm{XFC}(\mathrm{MXPTH}), \mathrm{JBF}(\mathrm{MXMEM})$

Data for the leg-to-leg transfer algorithm is stored in this block, along with matrix diffusion data. If path-averaged velocities are used, only the first entries of $\mathrm{NX}, \mathrm{NC}$, and $\mathrm{DX}$ are required.

$\mathrm{NX}$ - Number of grid blocks for each leg in the migration path, determined in DXDT and possibly refined in CHAIN. NX is used in SETUP, FACER, TRNSPT, and XCHNG.

NC - Number of catcher blocks for each leg in the migration path, determined in DXDT and possibly refined in CHAIN. NC is used in FACER, TRNSPT, and XCHNG.

DX - Grid block size for each leg in the migration path ( $f t$ ), read in FLOWIN and printed in INPRIN. Nonzero DX is checked in DXDT, otherwise, DX is determined in DXDT. Code-determined DX is potentially refined in CHAIN due to dimensioning and in SETUP to preserve the length of each leg. DX is used in FACER, $A D J B, M X C I, L$, and SETDIS. Note that DX(1) and DXX of COMMON XPORTB are identical for path-averaged velocity runs. 
TMN - Mean time spent over a transport time step as each isotope in each production subchain $(y)$, determined and printed in TIMER and used in SETUP and FACER.

BF - Transfer fractions for particles crossing into new legs over a transport time step, determined and printed in FACER and applied in TRNSPT.

JPSC - Pointers to blocks of data in JPN array, below, for each subchain. That is, for subchain $I$, all contributor and receiver block indicies for leg-to-leg transfer are found between entries JPN(JPSC(I) +1$)$ and JPN(JPSC(I+1)), where $\operatorname{JPSC}(1)=0$. JPSC is found and printed in FACER and used in TRNSPT.

JPN - Receiver and contributor block indicies from the leg-to-leg transfer algorithm. Block JPN $(I, 1)$ is contributed to over a time step from blocks $\operatorname{JPN}(I, 2)$ to $\operatorname{JPN}(I, 3)$, although with large Courant numbers there may be blocks in between these latter two indicies that do not contribute. JPN is determined and printed in FACER and used in TRNSPT.

IMB - Flag indicating presence (1) or not (0) of an immobile phase for each leg in the migration path, read in FLOWIN and printed in INPRIN. IMB is used in STOPPER, CHAIN, and XCHNG.

PHIM - Porosity in the immobile phase for each leg in the migration path, read in FLOWIN, checked in STOPPER, printed in INPRIN, and used in XCHNG.

XFC - Mass-transfer rates for each leg in the migration path (1/y), read in FLOWIN, checked in STOPPER, printed in INPRIN, and used in XCHNG.

$J B F$ - $J B F(I)$ is the index of the first transfer fraction in the $B F$ array for subchains ending in isotope I. JPF is found in FACER and used in TRNSPT.

COMMON/ XPORTA / RHO(MXGRD, MXMEM), RHONEW(MXGRD), F(NDDF),

1 SF(NDSF), RHOM (MXGRD, MXMEM)

This common contains several of the large chain- and grid-dependent arrays for the DVM transport model.

RHO - Amount (atoms) in the mobile phase for each grid block and for each isotope being transported, initialized to zero in METHOD and updated for each time step in TRNSPT, SRCIN, and XCHNG. Also used in TRNSPT for discharge calculations and atom count summaries.

RHONEW - Amount in the mobile phase for each grid block for a specific isotope being transported (atoms), initialized to zero in METHOD and used for temporary storage while updating the RHO array, above, in TRNSPT.

F - Discharge fractions over a transport time step for each subchain, found and printed in SETDIS and used in TRNSPT.

SF - Source distribution fractions over a transport time step for each subchain, found and printed in MXCLL and used in SRCIN. 
RHOM - Amount in the immobile phase for each grid block and for each isotope being transported (atoms), initialized to zero in METHOD and updated for each time step in XCHNG. Also used in TRNSPT for computing atom count summaries.

COMMON / XPORTB / B (2*MXNVI, MXSUB , MXPTH), JV(2*MXNVI, MXSUB , MXPTH),

$1 \mathrm{~V}$ (MXNVI, MXSUB, MXPTH), DP(MXSUB), N(MXSUB, MXPTH), JF(MXSUB+1),

2 LPS (MXMEM), LPE (MXMEM), LRP (MXSUB , 2), JSF (MXSUB+1), INSR (MXSUB),

3 DXX, INSRC, KFDT, KFDX(MXPTH), KFDXA, NSB, NTX, NVI

This common contains mostly arrays stacked by subchain for the DVM transport model.

B - Transport fractions for each subchain being transported for particles that remain in a leg over a transport time step, found and printed in SETUP and used in TRNSPT.

JV - Pointers to the transport fractions in array $B$, above, for each subchain being transported for particles that remain in a leg over a transport time step, found and printed in SETUP and used in TRNSPT. In particular, for subchain $J$ in leg $K$, block $I$ receives contribution from blocks $I$ - JV $(L, J, K)$ over a time step, where $L=1, \ldots, N(J, K)$ (see $N$ below). The fractions of the contributor blocks that wind up in block $I$ are found in $B(L, J, K)$, where $L=1, \ldots, N(J, K)$. Note that $K$ is set to one for path-averaged velocity runs.

$\mathrm{V}$ - Distributed velocities for each subchain being transported for each leg in the migration path ( $f t / y)$, found, used, and partially printed in SETUP and used in FACER, MXCLL, and SETDIS. In particular, $V(I, J, K)$ is the $I^{\text {th }}$ velocity for subchain $\mathrm{J}$ in leg $\mathrm{K}$. Note that $\mathrm{K}$ is set to one for path-averaged velocity runs.

DP - Decay survival fractions and production fractions over DT for each subchain. Here, DT, as described in COMMON MISC, can be TRLSE of COMMON OUTVEC, the time step for source calculations, or the time step for DVM transport calculations. DP is found in BRANCH using FUNCTION ET and is printed in SETUP, when DT is the transport time step. DP is used in SOURCE, DTUPDT, SETUP, TIMER, FACER, ADJB, and XCHNG.

$\mathrm{N}$ - Number of contributor blocks to any interior block in each leg of the migration path for each subchain being transported, found and printed in SETUP and used in TRNSPT. In particular, $\mathrm{N}(\mathrm{J}, \mathrm{K})$ is the number of contributor blocks in leg $\mathrm{K}$ for subchain $\mathrm{J}$. Note that $\mathrm{K}$ is set to one for path-averaged velocity runs.

$\mathrm{JF}$ - Pointers to sets of fractions for each subchain in the discharge factor array, $F$ of COMMON XPORTA, found in SETDIS and used in TRNSPT. In particular, the discharge fractions for subchain $I$ are stored contiguously from $F(J F(I)+1)$ to $F(J F(I+1))$.

LPS - LPS(I) points to the beginning of subchain information for subchains ending with isotope $I$, found in BRANCH and used in DTUPDT, SETUP, TIMER, FACER, MXCLL, TRNSPT, SRCIN, and XCHNG. 
For example, the decay survival fraction for isotope I over a time step is stored in $\operatorname{DP}(\operatorname{LPS}(I))$. LPS is also used as a locator for arrays B, JV, V, N, LRP, INSR, JF, and JSF described here and JPSC of COMMON XFER.

LPE - LPE(I) points to the end of subchain information for subchains ending with isotope I, found in BRANCH and used in DTUPDT, SETUP, TIMER, FACER, MXCLL, TRNSPT, SRCIN, and XCHNG. For example, the decay survival fraction for isotope $I$ over a time step, followed by the production fractions for all subchains ending in isotope $I$, are stored from DP(LPS(I)) to DP(LPE(I)). LPE is also used as a locator for arrays B, JV, V, N, LRP, INSR, JF, and JSF described here and JPSC of COMMON XFER.

LRP - Parent and subchain length for each subchain being transported, found in BRANCH and used in DTUPDT, SETUP, TIMER, FACER, ADJB, MXCLI, TRNSPT, SRCIN, and XCHNG. For subchain I, LRP $(I, 1)$ is the index of the first member of the subchain and $\operatorname{LRP}(I, 2)$ is the number of isotopes in the subchain.

JSF - Pointers to sets of fractions for each subchain in the source distribution array, SF of COMMON XPORTA, found and printed in MXCLL and used in SRCIN. In particular, the source distribution fractions for subchain $I$ are stored contiguously from $\operatorname{SF}(J \operatorname{SS}(I)+1)$ to $\operatorname{SF}(\mathrm{JSF}(I+1))$.

INSR - INSR(I) is the number of grid blocks in the up-gradient direction to receive source for subchain $I$, found in MXCLL and used in SRCIN. INSR is only used for the mixing-cell source distribution scheme and is used in conjunction with INSRC, described below.

DXX - Grid block size for transport for runs that use leg-averaged velocities ( $f t$ ), set to $\mathrm{DX}(1)$ by FLOWIN. Nonzero values are checked in DXDT, otherwise DXDT determines an appropriate value. Code-determined values of DXX may be further refined to preserve total path length in DXDT. DXX is used explicitly in MXCLL and implicitly as DX(1) in SETDIS.

INSRC - Index of the first block to receive source for runs that use the fluid flowthrough source model, determined in SETUP and used in SRCIN. If the mixing cell source model is used, INSRC is the index of the first non-catcher block. Source distribution for subchain I therefore starts with block INSRC INSR(I), where the latter array is described above.

KFDT - Flag indicating whether the user has specified (1) the transport time step for the decay chain being transported or not $(0)$, set in CHAIN and used in DXDT.

KFDX - KFDX(I) is a flag indicating whether the user has specified (1) the transport space step for leg $I$ of the transport path or not $(0)$, set in FLOWIN and used in CHAIN, DXDT, and SETUP.

KFDXA - Number of legs for which the user has specified the transport space step, set in FLOWIN and used in CHAIN, DXDT, and SETUP.

NSB - Number of grid blocks to receive the source injection calculated using the flowthrough source model, set to $\mathrm{NX}(1)$ by SETUP if the leg-to-leg transfer algorithm is used and set to the ratio of the length of the first leg of the migration path to the grid block size DXX by DXDT if leg-averaged velocities are used. For runs that use the mixing cell source model, NSB is set to zero in SETUP. NSB is used by SRCIN. 
- Total number of grid blocks, determined in CHAIN following the final refinements of the space steps and the catcher blocks. NTX is used in FACER, TRNSPT, and XCHNG.

NVI - Number of intervals over which the isotope velocities are distributed, set to the first increment determination parameter by FLOWIN, either from user-specified input or from the default value 7. NVI is used in DXDT, SETUP, FACER, ADJB, and MXCLL. 
The data input guide is divided into six sections. Section 4.1 describes the execution procedure for NEFTRAN II. Section 4.2 describes, in general terms, both the parameters and arrays required in the NEFTRAN II input file. Section 4.3 and Section 4.4 provide detailed descriptions of parameter and array input, respectively. The specific units required for the input data are discussed in sections 4.3 and 4.4 , but in general, the code requires data to be input in English Engineering system units. The input requirements for running multiple data sets are defined in Section 4.5 and a sample input file is discussed in Section 4.6.

\subsection{Execution Procedure for NEFTRAN II}

There are two versions of NEFTRAN II. The PC (personal computer) version has been compiled with Microsoft Fortran Version 2.3 and can be run on a personal computer. The PC memory requirements depend on the size of the arrays used by NEFTRAN II (see Section 3.3), which in turn, depends on the size of the problem being simulated.

To execute NEFTRAN II on a personal computer using the disk operating system (DOS), the user must type:

\section{NEFII \Pathname $\backslash$ Filename}

where "\Pathname\Filename" will be retrieved in subroutine FLOWIN and stored as a character string up to, but not including, the extension of "Filename." The character string (ROOT, used in FLOWIN and NEFMAIN) is then used to access other input files, if required, and to name output files as requested. Note that "Filename" need not have an extension, but it must be included on the command Iine if it does have an extension. Also, "\Pathnamel" must be included only if "Filename" exists in a directory different from the one containing the executable file (NEFII.EXE).

This execution procedure allows the user to use any name for the NEFTRAN II input file(s) and also allows the user to execute NEFTRAN II from one directory and write/access input/output files to another directory. For example, suppose the input file name is TEST.INP and resides in the same directory as the executable (NEFII.EXE). Then to execute this input file, the user would type:

NEFII TEST. INP

("\Pathname $\backslash$ " is not needed since the input file and the executable reside in the same directory). The naming of the auxiliary files would proceed as follows:

On Input (as required)

1. TEST.VEL (unit 11) must be the name of the data file containing time-dependent velocity fields.

2. TEST.QSC (unit 12) must be the name of the data file containing time-dependent flow rates through the source. 
3. TEST.SMP (unit 10) must be the name of the data file containing sampled data for over writing input.

On Output (as requested)

1. TEST.OUT (unit 6) is the name of the hardcopy output file written by NEFTRAN II.

2. TEST.DIS (unit 30 ) is the name of the output file containing discharge rates written by NEFTRAN II.

3. TEST.SRC (unit 25) is the name of the output file containing source rates written by NEFTRAN II.

This file naming scheme is useful for keeping track of input/output files that are required or generated by NEFTRAN II by ensuring consistency, and give the user a means of quality control.

For the VAX version of NEFTRAN II, the standard VAX computer execution procedure is required (i.e., RUN NEFII). The main difference between the VAX version and the $P C$ version is the file naming scheme. For the VAX version, the input file(s) must be named NEFII with the appropriate extension, instead of an arbitrary user input character string. For example, to run NEFTRAN II on the VAX, input must reside on a file named NEFII.INP. The auxiliary input files, as required, must be named NEFII.VEL, NEFII.QSC, and NEFII.SMP. The output files that NEFTRAN II would create, as required, would be named NEFII.OUT, NEFII.SRC, and NEFII.DIS. This required file naming procedure for the VAX version is equivalent to the PC version for ROOT equal to NEFII. INP. In the following discussion of input requirements, the name of the external files will be denoted (root) with the appropriate extension, in reference to the above $P C$ version file naming scheme.

\subsection{General Input Description}

All read statements for the input file are found in subroutine FLOWIN. Following the read of the 80-character string title, five sets of parameter data and five arrays are required. For the five arrays to be read, an array title and the actual data are required. Each row of the array is read on a separate record. Every set of parameters and array has a number of error checks in subroutine STOPPER.

Except for the character data (title and array names), all read statements are 1ist-directed. This helps avoid errors involving field width and alignment. However, because the read statements are list-directed, every parameter and array entry must be assigned a value. That is, use of a blank to indicate zero is not permissible. Currently there are up to 55 single-valued parameters that can be input to NEFTRAN II. Each parameter is read on a separate record. Using list-directed format, the value can be followed by a comma or blank and the rest of the record is ignored. If the user chooses to do so there is ample space to append a descriptive comment to each value. With this feature, and proper use of the array names, the input file can be generated in the form of a 
template. The use of a template allows the user to easily edit existing input files for data input changes and also minimizes references to a data input guide for a description of the input required. In sections $4.3,4.4$, and 4.5, for each set of data required, a sample input template is also provided.

If the user opts to include multiple data sets in a single simulation, the first record on the second and subsequent data sets contains flags indicating which of the ten input groups are to be read again. Since the title is not read again, the ten flags required consist of five flags for the parameter sets and five for the arrays. For array input, both the array names and array data must be supplied for all data sets. Data checking is also performed in STOPPER on the later data sets. Note that if external files are attached that contain supplemental input, they are re-wound and re-read for each data set, provided the option to use the file does not change.

\subsection{Parameter Input Description}

There are five sets of parameters that are input to NEFTRAN II. They include most of the options, dimension parameters, and physical parameters that appear in NEFTRAN II input file. Some parameters are derived from other input data. In particular, the option to implement the leg-to-leg transfer algorithm is automatically invoked if an external file of time-dependent velocities is used or if diffusion from mobile to immobile fluid occurs for any leg in the migration path. The latter criterion is used to decide whether the global diffusion flag is zero or nonzero. Also, two input parameters (LCH and MXC, described below) are used to determine an appropriate value for the source-type parameter IDSRC. The next five subsections describe the input for the five sets of parameter data.

\section{3 .1 Options}

Standard Print Options. One dummy title record and 11 records of single integer data. The standard print options are flags that indicate whether NEFTRAN II will print certain information to the hardcopy output file ( (root\}.out, unit 6). For each print option, zero means do not print and a nonzero integer implies print. The following discussion lists the information that is printed for each option. This information is considered standard in the sense that it is useful to the user when the code is executing without any problems. Note that options 2 - 6 echo the array input without any change of units.

\section{option Output Printed if Option is a Nonzero Integer}

IOPT(1) The complete set of parameters.

IOPT(2) The network leg properties array.

IOPT(3) The network junction properties array.

IOPT(4) The migration leg properties array. 
IOPT(5) The inventory array.

IOPT (6) The element properties array.

IOPT(7) Fluid velocities and source/discharge flow rates.

IOPT(8) Subchains, production factors, and velocities, only relevant for the DVM transport model.

IOPT(9) Space and time increments and source type, only relevant for the DVM transport model.

IOPT(10) Time-dependent output at the discharge point. If positive (e.g., $+\mathrm{N})$ then discharge rates $(\mathrm{C} i / \mathrm{y})$ are printed every $\mathrm{N}^{\text {th }}$ time step. If negative $(\mathrm{e} . \mathrm{g} .,-\mathrm{N})$, concentrations $\left(\mathrm{Ci} / \mathrm{ft}^{3}\right)$ are printed every $\mathrm{N}^{\text {th }}$ time step.

IOPT(11) Discharge summary (peak rates and integrated discharge).

An example of the template that can be used in the input file for these standard print options is given in Table 4.1.

External File options. One dummy title record and six single value integer records. In NEFTRAN II, external files are used to either store results (i.e., write-to) or for supplemental input (i.e., read-from). Specifically, external files are (1) used to store resultant source rates and discharge rates, (2) can be used to provide data to subroutine GETRV to over write selected input, usually with statistically sampled data, and (3) can be used to input time-dependent velocity fields and

Table 4.1.

Input Template for Standard Print Options

PARAMETERS, GROUP1 - OPTIONS, STANDARD PRINT, NONZERO $\Rightarrow$ PRINT

0 LIST OF ALL PARAMETERS

0 THE NETWORK LEG PROPERTIES ARRAY

0 THE NETWORK JUNCTION PROPERTIES ARRAY

0 THE MIGRATION PATH PROPERTIES ARRAY

0 THE DECAY CHAIN PROPERTIES ARRAY

0 THE ELEMENT PROPERTIES ARRAY

0 FLUID VELOCITIES \& SOURCE/DISCH FLOWS

0 SUBCHAINS, PRODUCTION \& VELOCITIES

0 SPACE \& TIME STEPS AND SOURCE TYPE

0 TIME DEPENDENT OUTPUT, IF $+/-N$, RATES/CONCS EVERY NTH TIME STEP

0 DISCHARGE SUMMARY 
time-dependent flow rates through the source regime. The following discussion will list each option and describe the external file that is either read-from or written-to. For all the external file options a zero implies not to write/read the external file and a nonzero integer indicates the opposite. The user should also note the added significance of the sign of option 13 .

\section{Option External File Description}

IOPT(12) If nonzero, write source rates to unit 25 . Note, unit 25 is opened by the code with the file name (root).SRC. The first four records written contain (1) title, (2) date and time of run, (3) data set number, trial number, and total number of isotopes (NTOT), and (4) isotope names. The last record written following each execution of the source model contains NTOT +1 zeroes. The records in between the first four and the last contain time (y) and NTOT source rates $(\mathrm{C} i / \mathrm{y})$. The first four records contain character data. All other records are written in list-directed format. This file is only written when the DVM transport model is implemented.

IOPT(13). If nonzero, write discharge rates to unit 30 . Unit 30 is opened with the name \{root\}.DIS. For this option, a nonzero integer has an additional meaning. If transport of multiple chains is simulated, each chain may be transported with a different time step, and, therefore, the discharge rates for each isotope may be found at different times. When the user inputs a positive integer for this option, NEFTRAN II interpolates the discharge rates found at different times and writes all the isotopes on the the same time scale. If this option is negative, NEFTRAN II writes the rates for each isotope sequentially by chain, at times corresponding to the time step used for each chain. In either case, the first two records consist of the title, and the execution date and time. The remaining format of \{root\}.DIS depends on whether the chains have been put on the same time scale or not. If they are on the same time scale, the remainder of the \{root\}.DIS file consists of the following records: (1) data set number, trial number, number of rates, and number of columns (i.e., total number of isotopes), (2) the name of each isotope, and (3) the simulation time (year) and the integrated discharge (Ci) for each isotope. Next, NEFTRAN II writes the time (year) versus discharge rate ( $\mathrm{Ci} / \mathrm{yr}$ ) information for each isotope, where the time is printed in the first column and the rate for each isotope is printed in each subsequent column. NEFTRAN II writes rates for each additional trial number and data set in the same format as described above. If the rates are not 
put on the same time scale for every chain, NEFTRAN II writes the data set number, the trial number and total number of chains. Next, NEFTRAN II writes the following records to \{root\}.DIS for each chain: (1) the chain number, the number of isotopes in the chain, and the number of times at which discharge rates are provided; (2) the name of each isotope in the chain; and ( 3 ) the simulation time and the integrated discharge for each isotope in the chain. The time versus discharge rate for each isotope in the chain is then written by NEFTRAN II. This format is repeated for each subsequent trial number and data set.

IOPT(14) Not currently used.

IOPT(15) If nonzero, read from an external file, attached as unit 10 with the name (root).SMP, usually containing statistically sampled data. This is done by calling subroutine GETRV to read unit 10 and to perform the user-defined instructions coded there. Subroutine GETRV reads from unit 10 in a list-directed format. The first two variables read are integers JTRIAL and NVAR. JTRIAL is the trial number and NVAR is the number of sampled variables to be read subsequently. Each sampled variable is then read which replaces a input variable that was read in FLOWIN from \{root\}. INP.

IOPT(16) If nonzero, read from an external file, attached as unit 11 and named (root).VEL, sets of fluid velocities that apply for discrete time frames. The data on unit 11 is read in a list-directed format by subroutine FLOWIN as illustrated:

$\begin{array}{rcrr}\mathrm{T}_{1} & \mathrm{~L}_{1} & \mathrm{~V}_{1,1} & \mathrm{~S}_{1,1} \\ & \mathrm{~L}_{2} & \mathrm{~V}_{1,2} & \mathrm{~S}_{1,2} \\ & \cdot & \cdot & \cdot \\ & \cdot & \cdot & \cdot \\ & \mathrm{I}_{2} & \mathrm{~V}_{1, \mathrm{~N}} & \mathrm{~S}_{1, \mathrm{~N}} \\ \mathrm{~T}_{2} & \mathrm{~L}_{1} & \mathrm{~V}_{2,1} & \mathrm{~S}_{2,1} \\ & \cdot & \cdot & \cdot \\ & \cdot & \cdot & \cdot \\ & \cdot & \cdot & \cdot\end{array}$

Here $\mathrm{N}$ stands for the number of legs in the migration path (see variable NPATH in section 4.3.2). $T_{1}$ indicates time $(y), L_{j}$ denotes the $j$ th $l e g$ in the migration path, $V_{i, j}$ is the pore fluid velocity $(f t / y)$, and $s_{1, j}$ is the saturation (dimensionless), for time frame $i$ and $\operatorname{leg} j$. It is assumed that the velocities and saturations associated with time $T_{i}$ 
apply from time $T_{1-1}$ to time $T_{1}$, where time $T_{0}$ is zero. It is required that $0<T_{1}<T_{2}<\ldots<T_{m}$, where $m$ is the number of time frames specified. The option to use this file is only available when the DVM transport model is being used and, as noted previously, automatically invokes the leg-to-leg transfer algorithm.

IOPT(17) If nonzero, read from an external file, attached as unit 12 and named \{root\}.QSC, sets of flow rates for source calculations that apply for discrete time frames. The data on unit 12 is read in a listdirected format by subroutine FLOWIN in time-flow rate pairs $\left(t_{1}, Q_{1}\right)$. Times must be increasing and cover the range of release time to simulation time. Flow rates must be non-negative. This option is only available when the DVM transport model is being implemented.

An example of the template that can be used in the input file for these options is given in Table 4.2.

Table 4.2.

Input Template for External File Options

EXTERNAL FILES, NONZERO $\rightarrow$ FILE WRITTEN/READ

0 WRITE SOURCE RATES ( $\mathrm{Ci} / \mathrm{Y}$ ) TO (root).SRC (UNIT 25)

0 WRITE DISCHARGE RATES ( $\mathrm{Ci} / \mathrm{y}$ ) TO (root).DIS, (UNIT 30)

0 NOT CURRENTLY USED

0 READ SAMPLED DATA FOR REPEATED TRIALS FROM \{root\}.SMP (UNIT 10)

0 READ TIME-DEPENDENT VELOCITIES FROM (root).VEL (UNIT 11)

0 READ TIME-DEPENDENT SOURCE FLOW RATES FROM \{root\}.QSC (UNIT 12)

Run Options. One dummy title record and six records of single value integer data. As indicated in previous sections, NEFTRAN II may be run by implementing several different models. The run-options are flags that indicate which models the user wishes to utilize. The following discussion will list each option and describe the model that is implemented depending on the integer value of the run option. If the user inputs run options that are not consistent (e.g., use mixing-cell source with the analytical solution transport model), a warning message will be printed to the hardcopy output files, but execution will continue. Note that option 22 is only applicable if there is no matrix diffusion and time-dependent velocities are not used. 


\begin{abstract}
Option Description
IOPT(18) If zero, use DVM to simulate transport. If nonzero, use the analytic solution.

IOPT(19) If zero, solve the flow network using the properties input for the network legs and junctions. If nonzero, use the pore velocities input in the Migration Path Array or time-dependent velocities from an external file, (see option 15 above). Note, if velocities are to be used from an external file, this option MUST be nonzero.

IOPT(20) The leach/solubility option can be 0,1 , or 2 to indicate to the source model to use leach rates only, solubilities only, or both, to determine source rates. This option is only applicable for the DVM transport mode1.

IOPT(21) The flow-through/mixing-cell option can be 0,1 , or 2 to indicate to the source model to use the flowthrough source, the mixing-cell source, or to choose one as appropriate to determine source rates. This option is only applicable for the DVM transport model.

IOPT(22) If zero, leaching occurs at a constant rate. If one, leaching occurs at an exponentially decaying rate. This option is only relevant for the DVM transport mode1.

IOPT(23) If nonzero, and other input does not impose its use, the leg-to-leg transfer algorithm will be imposed. This is only applicable when the DVM transport model is being implemented.
\end{abstract}

An example of the template that can be used in the input file for these options is given in Table 4.3.

Table 4.3.

Input Template for Run Control Options

RUN CONTROLS

- USE DVM (0) OR ANALYTIC SOLN (NONZERO)

O SOLVE NETWORK (0) OR INPUT VELOCITIES (NONZERO)

0 LEACH (0), SOLUBILITIES (1), OR BOTH (2) FOR SOURCE RATES

0 FLOWTHRU (0), MIXCELL (1), OR CHOOSE (2) FOR SOURCE RATES

0 CONSTANT (0) OR EXPONENTIAL (1) LEACH RATE MODEL

0 FORCE (NONZERO) USE OF LEG-TO-LEG TRANSFER ALGORITHM 
Debug Print Options. One dummy title record and 15 records of single value integer data. The debug print options are flags that indicate for NEFTRAN II to print debug information (i.e., information that can be used to isolate problems) to the hardcopy output file, (root\}.OUT. Consistent with the standard print options, a zero means do not print and a nonzero means print. If the user indicates to print debug information that is not consistent with the run options, as discussed below, a warning message will be printed to the hardcopy output file, and the information will not be printed, but execution will continue.

Option Debug Information Printed if Option is Nonzero

IOPT(24) The transport, discharge, and source distribution fractions (applicable for DVM transport only).

IOPT(25) Diagnostic information for subroutine BAND (applicable for the analytical solution transport model only).

IOPT(26) Diagnostic information for function GIT (applicable for the analytical solution transport model only).

IOPT(27) The coefficient matrix and constant vector used to solve for pressure at the leg junctions (relevant only if the flow network is solved).

IOPT(28) The input read from unit 10. for each call to GETRV (relevant only if option 15 is nonzero).

IOPT(29) Not currently used.

IOPT(30) The inventory at release time.

IOPT(31) Restrictions forced on the code-generated time steps for source and transport (applicable for the DVM transport model only).

IOPT(32) The leg-junction and the junction-leg connections (applicable only if the flow network is solved).

IOPT(33) The entire set of time-dependent velocities from the external file, unit 11 (relevant only for the DVM transport model).

IOPT(34) The atom count summary (applicable only for the DVM transport model).

IOPT(35) The pressure at each junction and flow rates through each leg (relevant only if the flow network is solved). 
IOPT(36) The leg-to-leg transfer and transport adjustment fractions (relevant only for the DVM transport model and if the leg-to-leg transfer algorithm is being used).

IOPT(37) The mean time spent as each isotope in each of the subchains as a fraction of the transport time step (only relevant for the DVM transport model).

IOPT(38) The time-dependent source flow rates (only applicable for the DVM transport model).

An example of the template that can be used in the input file for these options in given in Table 4.4.

Table 4.4.

Input Template for Debug Print Options

DEBUG PRINTS, NONZERO $\Rightarrow$ PRINT

0 DVM MULTIPLIERS FOR TRANSPORT, DISCHARGE, AND SOURCE

0 DIAGNOSTIC INFO FOR SUBROUTINE BAND

0 DIAGNOSTIC INFO FOR FUNCTION GIT

0 MATRIX/VECTOR SYSTEM SOLVED FOR FLOW

0 THE DATA READ FROM UNIT 10

O NOT CURRENTLY USED

0 INVENTORY AT RELEASE TIME

0 RESTRICTIONS PLACED ON THE TIME STEPS

0 LEG/JUNCTION \& JUNCTION/LEG CONNECTIONS

0 VELOCITY FIELDS FROM UNIT 11

0 THE ATOM COUNT SUMMARY

0 JUNCTION PRESSURES \& LEG FLOW RATES

0 LEG-TO-LEG TRANSFER FRACTIONS

0 TIME SPENT AS EACH ISOTOPE IN A SUBCHAIN

0 TIME-DEPENDENT SOURCE FLOW RATES

\subsubsection{Problem Size Parameters}

One dummy title record and five records of single value integer data, followed by two sets consisting of a dummy array title record and a record of multiple integer data. This set of parameters defines the size of the problem to be simulated by NEFTRAN II (e.g., number of legs, number of junctions, number of isotopes). These parameters must be less than the maximum dimensions allowed (see Section 3.3). The problem size parameters are read in two subsets, integer constants followed by integer arrays. The first subset is read like the options described above, one 
record per value. The second subset is read in two record-pairs. The first record of each pair is read as a character string, which can be used as an array title for the input template. The second record of the pair contains all the values of the one-dimensional array. The following discussion will list each parameter required and the definition of that parameter. Note that the decay chain inclusion flags are also read here since they help determine the problem size.

\section{Name $\quad$ Definition}

First Subset:

NLEG Number of legs in the network.

NJCT Number of junctions in the network.

NPATH Number of legs in the migration path.

NCHNS Number of decay chains to be input.

NELM Number of elements input (referenced in the Element Properties Array).

Second Subset (One-dimensional Arrays):

NI An array-title on the first record followed by NCHNS values on the second record, where each value indicates the number of isotopes in each decay chain.

INCHN An array-title on the first record followed by NCHNS values on the second record. Each value is either 0 , 1 , or 2, indicating that the decay chain is to be included in source calculations and transported, included in source calculations but not transported, or ignored for both, respectively.

The values for NLEG, NJCT, and NPATH are used to establish the rowdimensions of the Network Leg Properties Array, the Network Junction Properties Array, and the Migration Path Properties Array (all discussed in Section 4.4), respectively. If NJCT is input as zero, it is assumed that the network flow model will not be implemented and the reading of the Network Junction Properties Array is omitted. The Decay Chain Array (Section 4.4.4) has row-dimension equal to the total number of isotopes, found by summing over the NCHNS values of the NI array. The Element Properties Array (Section 4.4.5) has row-dimension equal to the product of NELM and NPATH.

The purposes of the INCHN array are for user-convenience and for reproducibility of source rates. For those applications that require a representation of a complete radionuclide inventory, the Decay Chain Array can be generated to include the entire representation. Then, all or selected decay chains can be transported for a given simulation by 
flagging appropriate entries in INCHN, which avoids editing of the Decay Chain Array. As discussed in Section 2.3.2, the source model of NEFTRAN II apportions isotope solubility according to the element solubility and the internally determined time-dependent mass fraction of the isotope to the element. Since the mass of the element at any time is the sum of masses of the isotopes of that element, source rates that depend on solubilities may differ depending on which decay chains are included. Source rates can be reproduced, therefore, by including the same decay chains in the source calculations, whether they are to be transported or not. One final note about the INCHN array is that an entry of 2 (the decay chain is not used for source or transport) for a chain causes modification of NCHNS and NI. That is, following the reading of the Decay Chain Array, NCHNS is decreased the appropriate amount, the NIarray is compacted, and the information for such a chain is NOT stored. These actions should not affect the user unless multiple data sets are provided, in which case the second data set cannot reference a decay chain that has been discarded ( $1 . e$. , not included for source or transport) in a previous data set. See section 4.5 for further discussion of multiple data sets. An example of the template that can be used in the input file for these options is given in Table 4.5.

Table 4.5.

Input Template for Problem Size Parameters

GROUP2 - PROBLEM SIZES

0 NUMBER OF NETWORK LEGS

0 NUMBER OF NETWORK JUNCTIONS

0 NUMBER OF MIGRATION PATH LEGS

0 NUMBER OF DECAY CHAINS INPUT

0 NUMBER OF ELEMENTS INPUT

CHAIN1 CHAIN2 CHAIN3 CHAIN4 CHAIN5 CHAIN6 - \# MEMBERS PER CHAIN 0

CHN1 CHN2 CHN3 CHN4 CHN5 CHN6 - SRC/TRANS-0, SOURCE-1, NEITHER-2

0

\subsubsection{Source and Flow Parameters}

One dummy title record and six records of single value floating point data. The physical parameters required on input, defined below, are primarily used to determine source rates. The exception is the last parameter, which is used to convert discharge rates to concentrations for output. 
ACSSFR The fraction of inventory accessed for source calculations.

VOL The pore volume $\left(f t^{3}\right)$ of the accessed source regime, used only if the mixing cell source is implemented.

QSAREA The product of cross-sectional area normal to flow and porosity (resulting units $-f t^{2}$ ). QSAREA is used to convert pore fluid velocity of the first leg in the migration path to fluid flow rate $\left(\mathrm{ft}^{3} / \mathrm{y}\right.$ ) through the source regime, and is used only if the network is not solved and either solubilities are accounted for or the mixing cell source is used. Note that if timedependent velocities are input, NEFTRAN II uses this parameter to create time-dependent flow rates through the source regime unless time-dependent flow rates through the source are also read from an external file.

RLCH The leach rate $\left(y^{-1}\right)$ used if the source is leachlimited.

SAL The density $\left(1 \mathrm{~b} / \mathrm{ft}^{3}\right)$ of fluid in the source regime, used to convert fluid mass to volume for solubility calculations and this value is used only if the fluid flow network is not solved.

QDAREA The product of cross-sectional area normal to flow and effective porosity (resulting units - $f t^{2}$ ), used to convert pore fluid velocity of the last leg in the migration path to fluid flow rate $\left(\mathrm{ft}^{3} / \mathrm{y}\right)$ at the discharge point. QDAREA is used only if the flow network is not solved and time-dependent radionuclide concentrations are requested for output (see Section 4.3.1).

If the network is solved (option 18 equals zero), QDAREA and SAL and QSAREA are determined internally. For time-varying flow fields, the flow rate at discharge will vary as the interstitial fluid velocity varies for the last leg in the migration path. Time-dependent flow rates through the repository are accounted for either by using the external timedependent velocity file (i.e., the velocity of the first leg in the migration path and QSAREA), or the flow rate will be updated periodically with flow rates read from an external file of time-dependent flow rates through the source ( $\{$ root $\} . Q S C)$, in which case QSAREA is not used. If the analytical transport option is chosen (option 17 is nonzero), variables ACSSFR, RLCH, and (optionally) QDAREA are used. In this case the leach time $(\tau)$ for the decaying band source boundary condition is taken to be the reciprocal of the leach rate, RLCH. An example of the template that can be used in the input for these options is given in Table 4.6 . 
Table 4.6.

Input Template for Source/Flow Parameters

GROUP3 - SOURCE/FLOW PARAMETERS

0 . FRACTION OF INVENTORY ACCESSED

0 . PORE VOLUME CONTAINING SOURCE ( $f t * * 3$ )

0 . PORE-AREA OF SOURCE ( $f t * * 2)$

0 . LEACH RATE ( $1 / y)$

0 . DENSITY OF SOURCE FLUID ( $1 \mathrm{~b} / \mathrm{ft} * * 3)$

0 . PORE-AREA AT DISCHARGE (ft**2)

\subsubsection{Time Parameters}

One dummy title record, four records of single value floating point data, a record containing a dummy array title, and a record with multiple single value floating point data. All time parameters required by NEFTRAN II are input in years. Like the Problem Size parameters, this set is separated into a subset of constants (in this case floating point constants) and a subset containing an array. The array must be preceded by a character record, which can be used as a descriptive title for the input template. The following discussion will list and describe each parameter required in this set.

Name Description

First Subset:

TUB The problem simulation time.

TRLSE The release time (i.e, the earliest time that contaminated fluid begins to migrate).

TLCH The time that leaching begins (cannot exceed TRLSE).

DTSRC The time step for source calculations. Entering zero indicates internal determination by NEFTRAN II.

Second Subset:

DTCHN An array title on the first record followed by NCHNS values on the second record, where each value is the time step for each decay chain input. The array title indicates which time step corresponds to which chain. Entering zero for a chain indicates internal determination of the time step by NEFTRAN II. 
For the analytical transport model (option 17 is nonzero), TRLSE and TLCH must be equal and DTSRC and DTCHN are not used. If TRLSE is input less than TLCH, NEFTRAN II sets TRLSE equal to TLCH and writes a warning message to the hardcopy output file ([root\}.OUT). For time-dependent velocities, it is important that the transport time step be an integer divisor of each time interval, and that internally determined time steps (which can vary from time interval to time interval) have this property. As discussed in Chapter 2, a time interval is defined as the beginning and ending time during which specific steady-state fluid flow velocities are assumed to occur in the migration path legs. Since the user can specify only a single value for all time, NEFTRAN II uses this value as a starting point for each time frame and then decreases it, as necessary, to ensure an integral divisor time step for the time interval. An example of the template that can be used in the input file for these options is given in Table 4.7 .

Table 4.7 .

Input Template for Time Parameters

\section{GROUP4 - TIME PARAMETERS}

$0 . \quad$ TIME TO END OF SIMULATION (y)

0 . TIME OF ONSET OF MIGRATION (y)

0 . TIME OF ONSET OF LEACHING ( $y$ )

0 . TIME STEP FOR SOURCE ( $y$ )

CHN1 CHN2 CHAIN3 CHAIN4 CHAIN5 CHAIN6 - TRANSPORT TIME STEPS BY CHAIN
0 . 0.
0 .
0 .
0 .
0 .

\subsubsection{Increment-Determination Parameters}

One dummy title record and 16 records of single value floating point data. Parameters that are used by NEFTRAN II for determining velocity increments, source time steps, transport time steps, and transport space steps are included here. These increment-determination parameters are used only for DVM transport (option 17 is zero) and all parameters have default values in NEFTRAN II. If the first parameter (INCRM) is zero, then all default values will be used and no further input for this data set is required. Otherwise, INCRM must be 16 and a value must be provided for each of the 16 parameters, although a zero indicates the use of the default value for the individual parameter. In the following discussion, after describing each parameter, the default values and recommended sets of values if the default values are not utilized will be presented. Any values different from those recommended should be selected very carefully based on extensive knowledge of the code. 
Following INCRM and the number of valocity intervals (NVI), meven parameters for finding the source time step, and elght parameter for finding the transport time and apace steps are listed. All are read as single-valued parameters, so descriptive mesage can follow each value for the input template. The following discussion will 11 st and describe each increment-determination parameter.

Name Description

INCRM If zero, use default values for all of the remaining parameters in this set and no further input is required for these parameters. Otherwise, a value of 16 must be input, which is the number of parameters (i.e., records) that follow.

NVI

The number of velocity intervals used to distribute velocities for the DVM transport model (default 7).

DTPAR(1) The minimum number of source time steps across the leach time, which is the reciprocal of the leach rate, RLCH (default 5).

DTPAR(2) The number, which when multiplied by an isotope's half-1ife, results in a time that must not exceed the isotope's mean migration time, if the isotope is to be considered important for determining the time step for source calculations (default 20 ).

DTPAR(3) The multiple of the minimum "important" half-life, providing a lower bound for the source time step (default 0.5 ).

DTPAR(4) The multiple of the mean mixing cell residence time to provide a lower bound for the source time step (default 0.2), only used if a mixing cell source is utilized.

DTPAR(5) The fraction of the minimum mean migration time taken over all important isotopes, which is then compared to mean mixing cell residence time, to decide (if optional) if the mixing cell source model should be used (default 0.1 ).

DTPAR(6) The fraction applied to the peak source flow rate to determine a source time step that captures the peak source flow rate (default 0.8). The fraction is multiplied by the peak source rate and this amount is added and subtracted from the peak rate. The times at which these two rates occur determines a time range used to bound the source time step. Only applied for time-varying flow rates through the source. 
DTPAR(7) The minimum number of time steps required to generate source rates (default 20).

DXDTPA(1) The number, which when multiplied by an isotope's half-life, results in a time that must not exceed the isotope's mean migration time, if the isotope is to be considered "important" for determining the time and space steps for transport (default 10).

DXDTPA(2) The number of standard deviations in time for important isotopes, providing a lower bound for the transport time step (default 1.5). This parameter is used only for the path-averaged transport to ensure resolution of the breakthrough curve in time.

DXDTPA(3) The maximum reduction factor that curve resolution restrictions can decrease the time step by (default $0.2)$. Only applied for path-averaged transport.

DXDTPA(4) The minimum number of time steps used for transport (default 30 ). For simulations that utilize timedependent velocities, 30 is divided by the ratio of total simulation time to the length of the current time frame. Either this number or 5 , whichever is greater, is used as the minimum number of time steps required for the current time frame.

DXDTPA(5) The Courant number to be used to initialize the space steps following determination of the time step (default 1.5).

DXDTPA(6) The minimum Courant number to be used if the first attempt at finding the space steps generates a number of space steps which exceeds dimensions (default $0.75)$.

DXDTPA(7) The minimum number of grid blocks for the pathaveraged DVM transport case (default 50).

DXDTPA(8). The minimum number of grid blocks for each leg for the leg-to-leg transfer implementation (default 7).

In regards to the above description of parameters, the concept of isotope importance is discussed in Section 2.3.2 and 2.4.3, and is based on the mean migration time for each isotope. If a decay chain is included in source but is not transported, all isotopes in that chain are considered to be "not important". Isotopes that do not discharge from their initial inventory during the simulation time have undefined mean migration time and are also considered "not important" in finding space and time steps. Further, if time-dependent velocities are used, all of the above parameters are applied to each time frame and there must be a nominal number of time steps across each time frame (refer to DXDTPA(4)). An example of the template that can be used in the input file for these options is given in Table 4.8 . 
Table 4.8 .

Input Template For Increment-Determination Parameters

GROUP5 - INCREMENT-DETERMINATION PARAMETERS

USE DEFAULTS (0) OR SUPPLY ALL (16) VALUES

\{or\}

GROUP5 - INCREMENT - DETERMINATION PARAMETERS

16 USE DEFAULTS (0) OR SUPPLY ALL (16) VALUES

0 \# VELOCITY INTERVALS (DEFAULT - 7)

0. MINIMUM \# OF SOURCE TIME STEPS ACROSS LEACH TIME (DEFAULT = 5)

0 . ISOTOPE IMPORTANCE PARAMETER FOR SOURCE DT (DEFAULT $=20$ )

0 . LOWER BOUND PARAMETER FOR SOURCE DT (DEFAULT $=.5$ )

0 . MIXING CELL PARAMETER FOR SOURCE DT (DEFAULT -.2 )

0 . PARAMETER TO DECIDE IF MIXING CELL USED (DEFAULT $=.1$ )

0 . PARAMETER TO ENSURE PEAK SOURCE FLOWRATE CAPTURE (DEFAULT $=.8$ )

0 . MINIMUM \# OF TIME STEPS FOR SOURCE (DEFAULT $=20$ )

0 . ISOTOPE IMPORTANCE PARAMETER FOR TRANSPORT DT (DEFAULT $=10$ )

0 . GURVE RESOLUTION PARAMETER FOR TRANSPORT DT (DEFAULT $=1.5$ )

0 . MAX. RED. IN TRANS. DT FOR CURVE RESOLUTION DT (DEFAULT $=0.2$ )

0 . MINIMUM \# OF TIME STEPS FOR TRANSPORT DT (DEFAULT $=30$ )

0 . THE INITIAL COURANT \# TO FIND DX'S (1.0)

0 . MINIMUM COURANT \# TO FIND DX'S (0.75)

0 . MINIMUM GRID BLOCKS FOR PATH-AVERAGED TRANSPORT (DEFAULT $=50$ )

0 . MINIMUM GRID BLOCKS/LEG FOR LEG-TO-LEG TRANSFER (DEFAULT $=7$ )

The space and time step parameters have default values in NEFTRAN II as indicated above and in sections 2.3.2, 2.4.3, and 2.4.4 These default values are based on the main purpose of NEFTRAN II, which is to be used in a performance assessment methodology (i.e., Monte Carlo statistical study). However, it is recognized that NEFTRAN II has the ability to be applied in a wide range of applications. Consequently, recommended sets of values for the increment-determination parameters that correspond to different levels of accuracy are given in Table 4.9. Leve1 1, which corresponds to the default values, provides coarse results and is usually adequate for rough analysis and statistical studies. Level 2 is the medium level accuracy and should serve well for generating breakthrough curves and statistical studies. Level 3 is the most accurate and the most time consuming, in general, and should, for the most part, be used only for deterministic runs where detailed breakthrough curves are required. To reiterate, although the user has the option to input any values for these parameters, it is recommended that only the sets of values listed in Table 4.9 be used. 
Table 4.9 .

Recommended Sets of Values

for the Increment-Determination Parameters

\begin{tabular}{llll}
\hline Parameter & $\begin{array}{c}\text { Level 1 } \\
\text { (default) }\end{array}$ & Level 2 & Level 3 \\
\hline DTPAR(1) & 5 & 10 & 25 \\
DTPAR(2) & 20 & 20 & 20 \\
DTPAR(3) & 0.5 & 0.3 & 0.1 \\
DTPAR(4) & 0.2 & 0.1 & 0.05 \\
DTPAR(5) & 0.1 & 0.1 & 0.1 \\
DTPAR(6) & 0.8 & 0.8 & 0.8 \\
DTPAR(7) & 20 & 35 & 50 \\
DXDTPA(1) & 10 & 10 & 10 \\
DXDTPA(2) & 1.5 & 0.7 & 0.35 \\
DXDTPA(3) & 0.2 & 0.1 & 0.05 \\
DXDTPA(4) & 30 & 50 & 60 \\
DXDTPA(5) & 1.5 & 1.8 & 2.0 \\
DXDTPA(6) & 0.7 .5 & 0.95 & 0.95 \\
DXDTPA(7) & 50 & 100 & 150 \\
DXDTPA(8) & 7 & 15 & 25 \\
& & & \\
\hline
\end{tabular}

\subsection{Array Input Description}

For each array that is input, an array title is included in the input file. The reason for this is to help keep track of data while editing input files using standard text editors. The row dimension for each array is specified by the Problem Size Parameters (Section 4.3.2). With the possible exception of the Junction Properties Array, a11 arrays are required input for the initial data set. If multiple data sets are provided, array input is required according to user specification. Both the title and the data must be supplied for any array that is to be read again in a new data set. Refer to Section 4.5 for further discussion on multiple data sets.

Leg properties are required in the first and third arrays. Certain properties are required to solve for flow in the network (e.g., hydraulic conductivity), certain properties are required for transport (e.g., connected porosity), and certain properties are required for both (e.g., leg length). If the pressure equations are not solved, many of the leg properties are not used. However, a value must be provided for each array entry because the input is read in a list-directed format. 


\subsubsection{Network Leg Properties Array}

Three dummy array title records followed by NLEG records containing seven data items. The name of the Network Leg Properties Array is read in three character-formatted records. These are followed by NLEG (Section 4.3.2) records, each containing the seven data items listed and described below. In the following discussion, column numeration is used to indicate a data entry on a record and not a specific column number on that record.

$\begin{array}{ll}\text { Column } & \text { Description } \\ 1 & \text { Leg number. } \\ 2 & \text { Inlet junction number. } \\ 3 & \begin{array}{l}\text { Outlet junction number, the sign convention in NEFTRAN } \\ \text { II for the flow direction is positive from the inlet } \\ \text { to outlet for each leg. }\end{array} \\ 4 & \text { Leg length (ft). } \\ 5 & \text { Cross-sectional area normal to flow (ft }{ }^{2} \text { ). } \\ 6 & \text { Hydraulic conductivity (ft/day). } \\ 7 & \text { Relative brine concentration (dimensionless), zero } \\ \text { implies fresh water and one implies saturated brine. }\end{array}$

If the network flow equations are not solved, columns 5,6 , and 7 are not used and any nonzero entry in these columns will prompt a warning message to be written to the hardcopy output file, but execution will continue. The inlet and outlet junctions are used in checking flow directions along the migration path and the leg lengths are required to find fluid and species migration times.

An array title that provides a template for input consists of column titles for each of the seven data items required. The column titles indicate data names (e.g., Hydraulic $K$ ) and required units (e.g., ft/d). An example of the template that can be used in the input file for this array is given in Table 4.10 . 
Table 4.10.

\section{Input Template for Network Leg Properties Array}

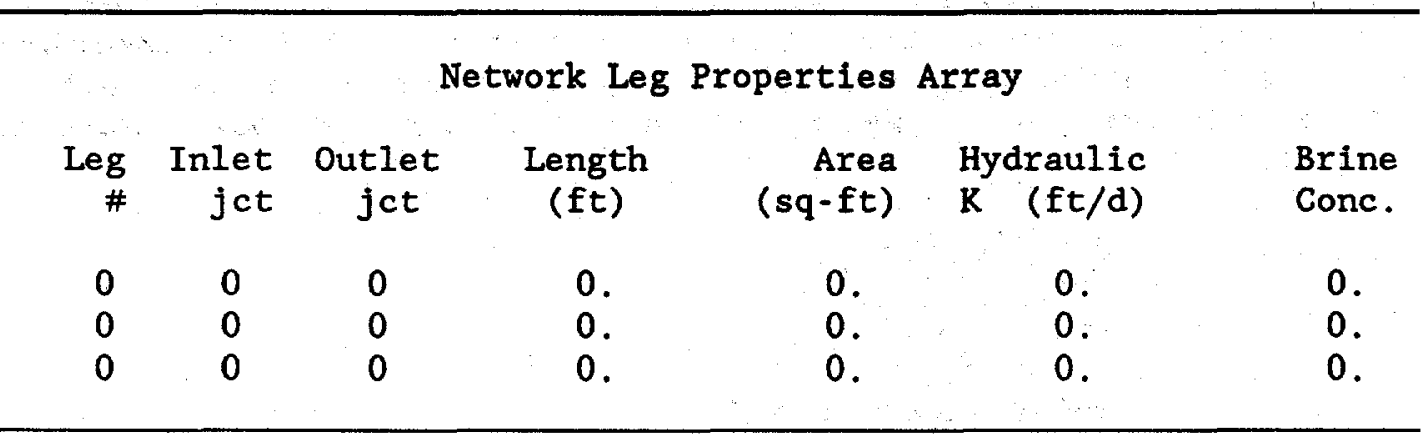

\subsubsection{Junction Properties Array}

Three dummy array title records followed by NJCT records each containing four data items. If the network flow system is not solved, the Junction Properties Array can be omitted. This is detected within NEFTRAN II by the value of NJCT (Section 4.3.2). If NJCT is zero, then the reading of the array-name and array data are bypassed. Otherwise, the array-name and array data are required. If the network is not solved, any nonzero entry in this array will prompt a warning message to be written to the output file, but execution will continue. The name of the Network Junction Properties Array is read in three character-formatted records. These are followed by NJCT records each containing the four data items listed and described below. In the following discussion, column numeration is used to indicate a data entry on a record and not a specific column number on that record.

$$
\begin{aligned}
& \text { Column Description } \\
& 1 \text { Junction number. } \\
& 2 \text { Elevation of the junction ( } \mathrm{ft} \text { ). } \\
& \text { 3. If zero, indicates that pressure is unknown at the } \\
& \text { junction, else the pressure from column number } 4 \text { is } \\
& \text { network. }
\end{aligned}
$$$$
\text { used as a known boundary pressure in solving the flow }
$$

An array title that provides a template for input by column consists of column titles for each of the four data items required. The column titles indicate data names (e.g., Pressure) and required units (e.g., psi). An example of the template that can be used in the input file for this array is given in Table 4.11. 
Table 4.11.

Input Template for Network Junction Properties Array

\begin{tabular}{cccc}
\multicolumn{5}{c}{ Network Junction Properties Array } \\
Jct & Elevation & Known Press? & Pressure \\
$\#$ & $(\mathrm{ft})$ & No/Yes $=0 / 1$ & (psi) \\
& & 0 & 0. \\
0 & 0. & 0 & 0. \\
0 & 0. & 0 & 0. \\
0 & 0. & 0 & 0.
\end{tabular}

\subsubsection{Migration Path Properties Array}

Three dummy array title records followed by NPATH records each containing eight data items. The name of the Migration Path Properties Array is read in three character-formatted records. These are followed by NPATH (Section 4.3.2) records each containing the eight data items listed and described below. In the following discussion, column numeration is used to indicate a data entry on a record and not a specific column number on that record.

\section{Column Description}

1 Leg number of the leg in the migration path, based on the leg numbering scheme of the Network Properties Array.

Longitudinal dispersivity $(\mathrm{ft})$.

Space step ( $f t)$. If input as zero, NEFTRAN II will determine the space step internally.

4 Flag indicating whether diffusion from the mobile phase to the immobile phase occurs in this leg (i.e., whether the matrix diffusion model is to be implemented for this $(\mathrm{eg})$, zero implies no and nonzero implies yes.

Interstitial porosity for the mobile phase.

$6 \quad$ Porosity for the immobile phase.

7 Mass-transfer coefficient for diffusion $\left(y^{-1}\right)$.

8 Interstitial fluid velocity $(\mathrm{ft} / \mathrm{y})$ 
An array title that provides a template for input by column consists of column titles for each of the eight data items required. The column titles indicate data names (e.g., Velocity) and required units (e.g., $\mathrm{ft} / \mathrm{y})$. An example of the template that can be used in the input file for this array is given in Table 4.12.

Table 4.12.

Input Template for Migration Path Properties Array

\begin{tabular}{|c|c|c|c|c|c|c|c|}
\hline \multirow[b]{2}{*}{$\begin{array}{c}\text { Leg } \\
\#\end{array}$} & \multicolumn{5}{|c|}{ Migration Path Properties } & \multicolumn{2}{|c|}{ Array } \\
\hline & $\begin{array}{l}\text { Disp. } \\
\text { (ft) }\end{array}$ & $\begin{array}{l}D X \\
(f t)\end{array}$ & $\begin{array}{l}\text { Diffus? } \\
\mathrm{N} / \mathrm{Y}=0 / 1\end{array}$ & $\begin{array}{l}\text { Mobile } \\
\text { Poros. }\end{array}$ & $\begin{array}{l}\text { Immob } \\
\text { Poros }\end{array}$ & $\begin{array}{l}\text { Mass Xfer } \\
\text { Coef }(1 / y)\end{array}$ & $\begin{array}{c}\text { Velocity } \\
(\mathrm{ft} / \mathrm{y})\end{array}$ \\
\hline 0 & 0 . & 0 . & 0 & 0. & 0. & 0. & 0. \\
\hline 0 & 0. & 0. & 0 & 0. & 0. & 0. & 0 . \\
\hline 0 & 0. & 0. & 0 & 0. & 0. & 0. & 0 \\
\hline
\end{tabular}

To understand the required input of the Migration Path Array, the different run option combinations that NEFTRAN II is capable of implementing must be understood. In NEFTRAN II, there are ten run option combinations as shown in Table 4.13.

Table: 4.13 .

Run Option Combinations

\begin{tabular}{lllll}
\hline $\begin{array}{l}\text { Network } \\
\text { Solved? }\end{array}$ & $\begin{array}{c}\text { External } \\
\text { Velocities? }\end{array}$ & $\begin{array}{c}\text { Solution } \\
\text { Technique }\end{array}$ & $\begin{array}{l}\text { Matrix } \\
\text { Diffusion? }\end{array}$ & $\begin{array}{l}\text { Force } \\
\text { L-to-L } \\
\text { Transfer? }\end{array}$ \\
\hline Yes & No & DVM & No & No \\
Yes & No & DVM & No & Yes \\
Yes & No & DVM & Yes & Default, Yes \\
Yes & No & Anl & No & NA \\
No & No & DVM & No & No \\
No & No & DVM & No & Yes \\
No & No & DVM & Yes & Default, Yes \\
No & No & An1 & No & NA \\
No & Yes & DVM & No & Default, Yes \\
No & Yes & DVM & Yes & Default, Yes \\
& & V & S & \\
\hline
\end{tabular}


The first three columns in Table 4.13 are specified in the run option parameters of Section 4.3.1, which are option numbers 19, 16, and 18 , respectively. Colum number 4 is declded based on the diffusion flags for all of the legs (column number 4 in the Migration Path Array). The last column is also specified in the run option parameters and is option 23. In conjunction with these different run option combinations, three assumptions contained in NEFTRAN II should be noted. First, the analytical transport model is not equipped to treat matrix diffusion nor time-dependent velocities. Second, if matrix diffusion is implemented for any leg in the migration path or if an external file of velocities is used, then the DVM leg-to-leg transfer algorithm is automatically invoked. Third, implementation of the leg-to-leg algorithm can be forced using option 23. Forcing leg-to-leg transport if matrix diffusion is occurring or if time-dependent velocities are being used is not necessary because it is already forced and is listed in Table 4.13 as being "default YES" for these run options. Table 4.14 outlines which columns (and in some cases rows) are used in the Migration Path Array for the specific run-option combinations listed above.

4.4.4 Decay Chain Array

Three dummy array title records followed by NTOT records each containing eleven data items.

Table 4.14.

Migration Path Array Parameters as a Function of Run Option

I. If the NETWORK IS SOLVED

- Leg numbers are used.

- Mobile phase porosities are used.

- At least one value (see below) of dispersivity is used.

A. For the ANALYTICAL SOLUTION

- Only one value of dispersivity is used and this is taken from the first row in the array (i.e. the dispersivity for the first leg).

B. For the DVM SOLUTION

- The flags indicating diffusion/no diffusion and the option forcing the leg-to-leg transfer algorithm are used.

a. If there is NO DIFFUSION and LEG-TO-LEG TRANSFER has not been forced 
- Transport is calculated over a property-averaged path that only one value of dispersivity and one space step are used, and these are taken from the first row in the array.

b. If there is NO DIFFUSION and LEG-TO-LEG TRANSFER has been forced

- Transport is calculated over each leg; therefore, for each leg, a dispersivity and a space step, are used.

c. If there IS DIFFUSION

- Transport is calculated over each leg; therefore, for each leg, a dispersivity, a space step, an immobile phase porosity, and a mass transfer coefficient are used.

II. If the NETWORK IS NOT SOLVED AND AN EXTERNAL FILE IS NOT USED

1. Leg numbers are used.

2. Fluid velocities are used.

3. At least one value (see below) of dispersivity is used.

A. For the ANALYTICAL SOLUTION

- Only one value of dispersivity is used and it is taken from the first row (i.e. the dispersivity for the first leg).

B. For the DVM SOLUTION

- The flags indicating diffusion/no diffusion and the option to force leg-to-leg transfer are used.

a. If there is NO DIFFUSION and LEG-TO-LEG TRANSFER has not been forced

- Transport is calculated over a property averaged path so that only one value of dispersivity and one space step are used, and these are taken from the first row. 
Table 4.14. (Concluded)

Migration Path Array Parameters as a Function of Run Option

b. If there is NO DIFFUSION and LEG-TO-LEG TRANSFER has been forced

- Transport is calculated over each leg; therefore, for each leg, a dispersivity and a space step, are used.

c. If there IS DIFFUSION

- Transport is calculated over each leg; therefore, for each leg, a dispersivity, a space step, a mobile phase porosity, an immobile phase porosity, and a mass transfer coefficient are used.

III. If the NETWORK IS NOT SOLVED AND AN EXTERNAL FILE IS USED

1. Leg numbers are used.

2. Dispersivities are used.

3. Space steps are used.

4. DVM SOLUTION is required.

5. The flags indicating diffusion/no diffusion and the flag to force leg-to-leg transfer are used.

a. If there IS DIFFUSION

- For each leg; a mobile phase porosity, an immobile phase porosity, and a mass transfer coefficient are used.

The name of the Decay Chain Array is read in three character-formatted records. These are followed by NTOT records each containing the eleven data items listed and described below. Here, as in the code, NTOT represents the total number of isotopes input, found by summing the number of isotopes over decay chains. In the following discussion, column numeration is used to indicate a data entry on a record and not a specific column number on that record.

$\underline{\text { Column }}$

1

\section{Description}

Isotope name, in this special case the name MUST be surrounded by single quotes to enable FORTRAN listdirected character input. 
Atomic mass, used only for solubility calculations and can be set to the atomic number.

3

4

5

6

8

9

10

11

Element index, should match an element index in the Element Properties Array below.

Local index, refers to index within the decay chain and is used to reference parent(s).

Local index of parent number 1 .

Local index of parent number 2 , cannot be nonzero unless column 5 is nonzero.

Branching fraction from the decay of parent \# 1 .

Branching fraction from the decay of parent \#2.

The initial inventory (Ci).

The half-life (y).

Weighting factor that divides into total integrated discharge for each isotope. This may be useful for performance assessment applications, where the appropriate weighting factor for each isotope may be found based on the EPA Containment Requirements (EPA, 1985 ), and the resultant weighted integrated discharges would be equivalent to EPA sums.

The element index for all isotopes of a given element should be the same so that solubilities will be treated correctly in the source calculations. The same element retardation is assigned to all isotopes of the element. If weighting factors are input as zero, the default value of 1.0 is used.

An array title that provides a template for input by column consists of column titles for each of the eleven data items required. The column titles indicate data names (e.g., Inventory) and required units (e.g., Ci). An example of the template that can be used in the input file for this array is given in Table 4.15.

\subsubsection{Element Properties Array}

Three dummy array title records followed by NELM record-sets containing NPATH records. The name of the Element Properties Array is read in three character-formatted records. These are followed by NELM (Section 4.3.2) record-sets, each pertaining to a specified element and each containing NPATH (Section 4.3.2) records. The first record of a set contains element index, solubility, the index of the first leg in the migration - path, the mobile phase retardation factor $\left(R_{d}\right)$ for the first leg in the migration path, and the immobile phase $R_{d}$ for the first leg in the migra- 
Table 4.15 .

Input Template for Decay Chains Array

\begin{tabular}{|c|c|c|c|c|c|c|c|c|c|c|}
\hline \multirow[b]{2}{*}{$\begin{array}{l}\text { Name } \\
\text { A6 }\end{array}$} & \multirow[b]{2}{*}{$\begin{array}{l}\text { Atom } \\
\text { Mass }\end{array}$} & \multirow[b]{2}{*}{$\begin{array}{l}\text { Ele- } \\
\text { ment }\end{array}$} & \multicolumn{6}{|c|}{ Decay Chains Array } & \multirow[b]{2}{*}{$\begin{array}{c}\text { Hf-Lf } \\
(y)\end{array}$} & \multirow[b]{2}{*}{$\begin{array}{c}\text { Weigh } \\
\text { Fac }\end{array}$} \\
\hline & & & $\begin{array}{l}\text { Loc } \\
\text { ndx }\end{array}$ & $\begin{array}{r}\text { Par } \\
\# 1\end{array}$ & $\begin{array}{r}\text { Par } \\
\# 2\end{array}$ & $\begin{array}{l}\text { Frac } \\
\text { From1 }\end{array}$ & $\begin{array}{l}\text { Frac } \\
\text { From2 }\end{array}$ & $\begin{array}{r}\text { Inven } \\
\text { (Ci) }\end{array}$ & & \\
\hline $\begin{array}{l}\text { 'name1' } \\
\text { 'name2' } \\
\text { 'name3' }\end{array}$ & $\begin{array}{l}0 \\
0 \\
0\end{array}$ & $\begin{array}{l}0 \\
0 \\
0\end{array}$ & $\begin{array}{l}0 \\
0 \\
0\end{array}$ & $\begin{array}{l}0 \\
0 \\
0\end{array}$ & $\begin{array}{l}0 \\
0 \\
0 .\end{array}$ & $\begin{array}{l}0 . \\
0 . \\
0 .\end{array}$ & $\begin{array}{l}0 . \\
0 . \\
0 .\end{array}$ & $\begin{array}{l}0 . \\
0 . \\
0 .\end{array}$ & $\begin{array}{l}0 . \\
0 . \\
0 .\end{array}$ & $\begin{array}{l}0 . \\
0 . \\
0 .\end{array}$ \\
\hline
\end{tabular}

tion path. Records 2 through NPATH of a set contain the leg index, the mobile phase $R_{d}$, and the immobile phase $R_{d}$ for legs 2 through NPATH, respectively. Every element index referenced in the Decay Chains Array above should be included in the first column of the Element Properties Array. Each column required in the Element Properties Array is listed below, along with a description of that column. In the following discussion, column numeration is used to indicate a data entry on a record and not a specific column number on that record.

\section{Column Description}

First Record:

1 Index of the element.

2 Solubility (g-element/g-fluid).

3 Index of the first leg in the migration path.

4 Mobile $\mathrm{R}_{\mathrm{d}}$ for the first $\mathrm{leg}$ in the migration path.

5 Immobile $R_{d}$ for the first leg in the migration path.

$I^{\text {th }}$ Record, $I=2, \ldots$, NPATH:

1 Index of the $I^{\text {th }}$ leg in the migration path.

2 Mobile $R_{d}$ for the $I^{\text {th }}$ leg in the migration path.

3 Immobile $R_{d}$ for the $I^{\text {th }}$ leg in the migration path

Note that the record-set described above must be repeated for each element. Solubilities are not used if only leaching is considered in finding the source rate (option 20, section 4.3.1). Immobile phase $R_{d}$ is 
not used unless diffusion is being modeled. If nonzero values are input for these parameters for these cases, a warning message is written to the hard copy output file, but execution continues. However, it should be reiterated that, since the array is input in a list-directed format, a blank is not accepted as input for these parameters.

An array title that provides a template for input by column consists of column titles for each of the data items required. The column titles indicate data names (e.g., Solubility) and required units $(e . g ., \mathrm{g} / \mathrm{g}$ ). -An example of the template that can be used in the input file for this array is given in Table 4.16 .

Table 4.16 .

Input Template for Element Properties Array

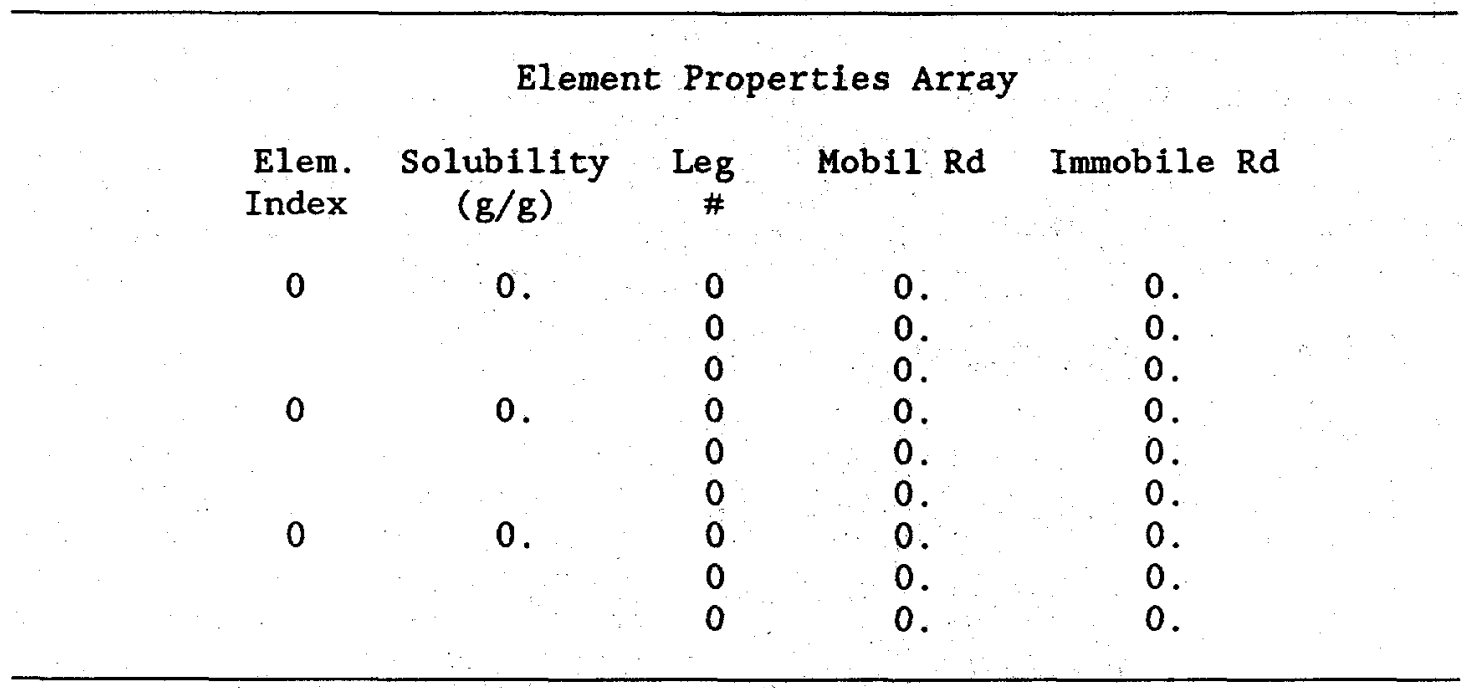

\subsection{Data Required for Multiple Simulations}

For a single run of NEFTRAN II, the run-title, the five sets of parameters, and the five arrays described are required in the input file. Multiple runs of NEFTRAN II can be accomplished using two methods. First, multiple data sets can be included sequentially on the input file. Second, an external file can be read and user-specified replacement statements implemented. In fact, although not routinely done, these two methods can be used together. The two methods are described in the next two subsections.

\subsubsection{Multiple Data Sets}

One dummy title record and one multiple integer record. For NEFTRAN II, the initial data set is read, and following transport of all chains, the code returns to subroutine FLOWIN to read additional data. If an end-of- 
file is encountered on the input file, the program terminates normally. Otherwise, the code reads the INP array, which contains flags that indicate which data groups are to be re-read. In NEFTRAN II there are 10 data groups that can be flagged. The basic idea is to provide the capability to perform additional calculations without having to re-input those data groups that do not change.

For NEFTRAN II the INP array is read in two records. The first record is a character record that can contain a descriptive title for the second record. The second record contains 10 integers, the first five for the parameter sets and the second five for the arrays. A nonzero entry signifies that the corresponding data-group should be re-read. The following discussion lists each entry in the INP-array and the corresponding data-group that is read again if the entry is nonzero.

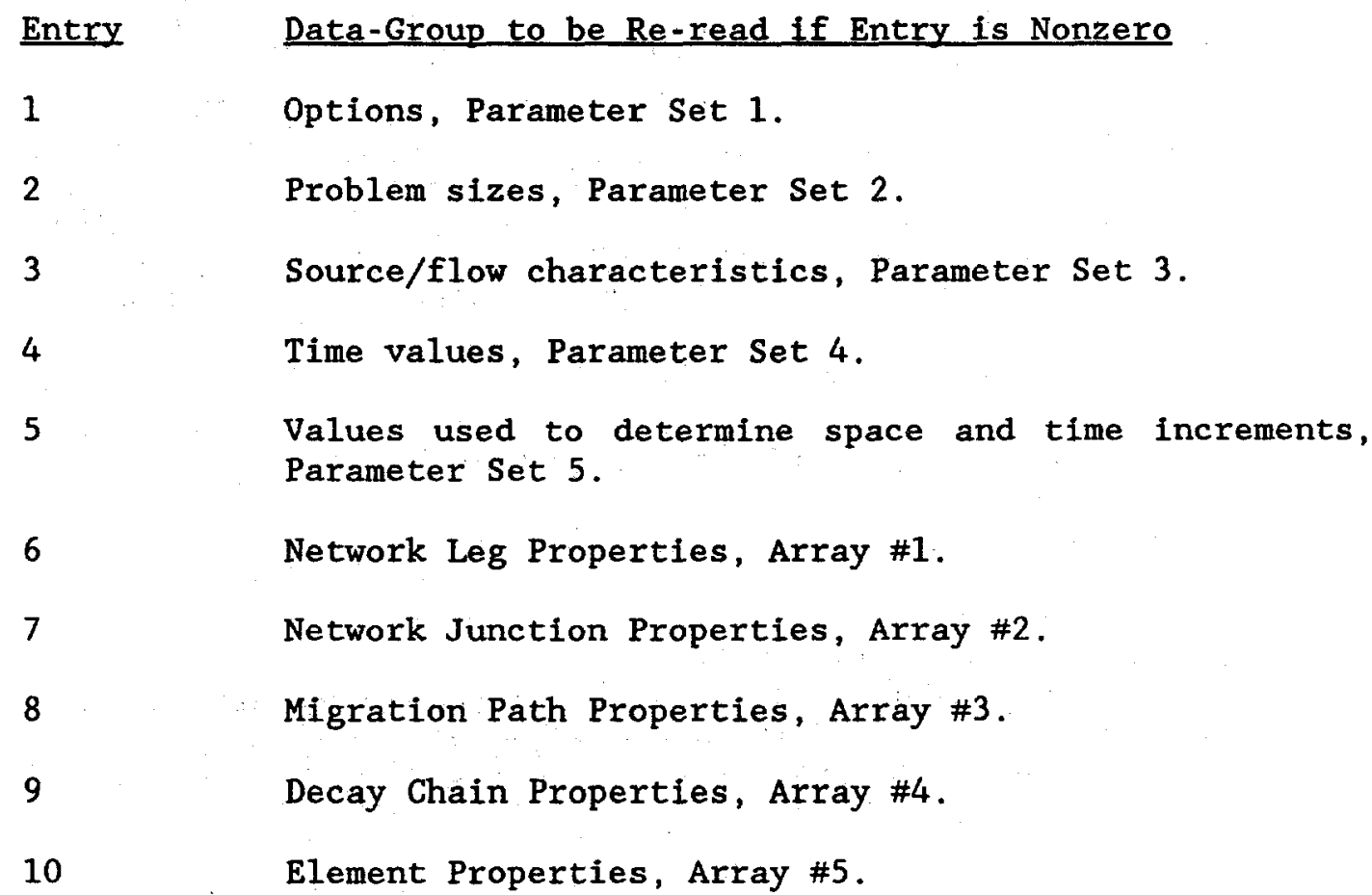

An example of a template that can be used in the input file for this array is given in Table 4.17. The array title consists of column titles for each data group (e.g., set one, set two, array one, array two).

Table 4.17.

Input Template for Multiple Data Set Options

SET1 SET2 SET3 SET4 SET5 ARY1 ARY2 ARY3 ARY4 ARY5 RE-READ FLAGS 1
0
0
0
0

0

$\begin{array}{llll}0 & 0 & 0 & 0\end{array}$ 
All data-items are subject to error checking during the input of a second or later data set. In addition, if all data groups are not re-read, subroutine STOPPER is called to re-check the entire current data set. This should trap most errors. However, it is recommended that any change in Problem Sizes be accompanied by re-reading all of the affected arrays. In particular, unintended information can be accessed if a decay chain was not included on an earlier data set (see the INCHN Array, Section 4.3.2) and is included in source and/or transport for a later data set.

It should be noted that any external input files are re-wound and re-read for each data set, as long as the option to include such files has not changed. For (root).SMP this means that a second set of repeated trials can be run with different data sets. For (root).VEL and (root).QSC, the re-reading is done mainly for Internal code convenience.

\subsubsection{Use of Subroutine GETRV}

NEFTRAN II allows for the use of an external file to over-write data that was read in the input file. The logic is to first read an input file and, if option 15 is nonzero, to then call subroutine GETRV to (1) read a record from unit 10 (\{root\}.SMP) and (2) implement the user-defined replacement statements that equate NEFTRAN II variable names to the data from unit 10 .

In NEFTRAN II, \{root\}.SMP is read until an end-of-file marker is encountered. At that time the program returns to subroutine FLOWIN to read another data set. If one is encountered, unit 10 is re-wound and is ready for a second set of repeated trials. The required structure of unit 10 is discussed in Section 4.3.1.

NEFTRAN II calls subroutine STOPPER following the users replacement statements in subroutine GETRV. In this manner, data checking is available in NEFTRAN II that augments any user-defined data checks defined by the user in GETRV.

\subsection{Sample Input File}

The purpose of this section is to present a sample input file to summarize the information that has been presented in sections $4.2-4.5$. Consequently, a sample input file for an arbitrary problem is shown in Table 4.18. Specific sample problems and their corresponding input will be presented in Chapter 5. As shown in Table 4.18, the first record in the input file contains the title for the simulation, "NEFTRAN II SAMPLE INPUT FILE". For the options, all standard prints are requested and two debug prints are requested. From the second subset of options, both source and discharge rate files are to be written. From the run controls, the code is instructed to use the DVM transport model, the pore velocities input in the Migration Path Properties Array, and to consider leaching using a constant leach rate model with a flow-through source, ignoring solubilities and mixing cell effects. 
Table 4.18 .

Sample Input File

NEFTRAN II SAMPLE INPUT FILE
PARAMETERS, GROUP1 - OPTIONS, STANDARD PRINT, NONZERO $\rightarrow$ PRINT

1 LIST OF ALL PARAMETERS

1 THE NETWORK LEG PROPERTIES ARRAY

1 THE NETWORK JUNCTION PROPERTIES ARRAY

1 THE MIGRATION PATH PROPERTIES ARRAY

1 THE DECAY CHAIN PROPERTIES ARRAY

1 THE ELEMENT PROPERTIES ARRAY

1 FLUID VELOCITIES \& SOURCE/DISCH FLOWS

1 SUBCHAINS, PRODUCTION \& VELOCITIES

1 SPACE \& TIME STEPS AND SOURCE TYPE

1 TIME DEPENDENT OUTPUT, IF + $\mathrm{N}$, RATES/CONCS EVERY NTH TIME STEP

1 DISCHARGE SUMMARY

EXTERNAL FILES, NONZERO $\rightarrow$ FILE WRITTEN/READ

1 WRITE SOURCE RATES ( $\mathrm{Ci} / \mathrm{y}$ ) TO \{root\}.SRC (UNIT 25)

1 WRITE DISCHARGE RATES (Ci/y) TO (root).DIS, (UNIT 30)

0 NOT CURRENTLY USED

0 READ SAMPLED DATA FOR REPEATED TRIALS FROM (root).SMP (UNIT 10)

0 READ TIME-DEPENDENT VELOCITIES FROM (root\}.VEL (UNIT 11)

0 READ TIME-DEPENDENT SOURCE FLOWRATES FROM (root).QSC (UNIT 12)

RUN CONTROLS

0 USE DVM (0) OR ANALYTIC SOLN (NONZERO)

1 SOLVE NETWORK (0) OR INPUT VELOCITIES (NONZERO)

0 LEACH (0), SOLUBILITIES (1), OR BOTH (2) FOR SOURCE RATES

0 FLOWTHRU (0), MIXCELL (1), OR CHOOSE (2) FOR SOURCE RATES

0 CONSTANT (0) OR EXPONENTIAL (1) LEACH RATE MODEL

0 FORCE (NONZERO) USE OF LEG-TO-LEG TRANSFER ALGORITHM

DEBUG PRINTS, NONZERO $\Rightarrow$ PRINT

0 DVM MULTIPLIERS FOR TRANSPORT, DISCHARGE, AND SOURCE

0 DIAGNOSTIC INFO FOR SUBROUTINE BAND

0 DIAGNOSTIC INFO FOR FUNCTION GIT

0 MATRIX/VECTOR SYSTEM SOLVED FOR FLOW

0 THE DATA READ FROM UNIT 10

O NOT CURRENTLY USED

1 INVENTORY AT RELEASE TIME

1 RESTRICTIONS PLACED ON THE TIME STEPS

0 LEG/JUNCTION \& JUNCTION/LEG CONNECTIONS

0 VELOCITY FIELDS FROM UNIT 11

0 THE ATOM COUNT SUMMARY

0 JUNCTION PRESSURES \& LEG FLOWRATES

0 LEG-TO-LEG TRANSFER FRACTIONS

0 TIME SPENT AS EACH ISOTOPE IN A SUBCHAIN

0 TIME-DEPENDENT SOURCE FLOWRATES 
Table 4.18 .

Sample Input File (Continued)

GROUP2 - PROBLEM SIZES

3 NUMBER OF NETWORK LEGS

O NUMBER OF NETWORK JUNCTIONS

3 NUMBER OF MIGRATION PATH LEGS

1 NUMBER OF DECAY CHAINS INPUT

3 NUMBER OF ELEMENTS INPUT

CHAIN1 CHAIN2 CHAIN3 CHAIN4 CHAIN5 CHAIN6 - \# MEMBERS PER CHAIN 3

CHN1 CHN2 CHN3 CHN4 CHN5 CHN6 - SRC/TRANS-0, SOURCE-1, NEITHER-2

0

GROUP3 - SOURCE/FLOW PARAMETERS

$1.0 \quad$ FRACTION OF INVENTORY ACCESSED

0 . PORE VOLUME CONTAINING SOURCE ( $f t * * 3$ )

0 . PORE-AREA OF SOURCE ( $f t * * 2)$

$1.0 \mathrm{e}-4$ LEACH RATE ( $1 / \mathrm{y})$

0 . DENSITY OF SOURCE FLUID ( $1 \mathrm{~b} / \mathrm{ft} * * 3$ )

0 . PORE-AREA AT DISCHARGE (ft**2)

GROUP4 - TIME PARAMETERS

1.0e4 TIME TO END OF SIMULATION (y)

0 . TIME OF ONSET OF MIGRATION $(y)$

0 . TIME OF ONSET OF LEACHING $(y)$

0 . TIME STEP FOR SOURCE ( $y$ )

CHN1 CHN2 CHAIN3 CHAIN4 CHAIN5 CHAIN6 - TRANSPORT TIME STEPS BY CHAIN 0 .

GROUP5 - INCREMENT DETERMINATION PARAMETERS

0 USE DEFAULTS (0) OR SUPPLY ALL (16) VALUES

Network Leg Properties Array

\begin{tabular}{|c|c|c|c|c|c|}
\hline $\begin{array}{c}\text { Leg } \\
\#\end{array}$ & $\begin{array}{c}\text { Inlet } \\
\text { jet }\end{array}$ & $\begin{array}{l}\text { Outlet } \\
\text { jct }\end{array}$ & $\begin{array}{l}\text { Length } \\
\text { (ft) }\end{array}$ & $\begin{array}{r}\text { Area } \\
(s q-f t)\end{array}$ & $\begin{array}{l}\text { Hydraulic } \\
\mathrm{K} \quad(\mathrm{ft} / \mathrm{d})\end{array}$ \\
\hline 1 & 1 & 2 & 50 & 0. & 0. \\
\hline 2 & 2 & 3 & 1000 & 0 . & 0. \\
\hline 3 & 3 & 4 & 2000 & 0. & 0 \\
\hline
\end{tabular}

Migration Path Properties Array

$\begin{array}{cccccccc}\begin{array}{c}\text { Leg } \\ \#\end{array} & \begin{array}{c}\text { Disp. } \\ (\mathrm{ft})\end{array} & \begin{array}{c}\text { DX } \\ (\mathrm{ft})\end{array} & \begin{array}{c}\text { Diffus? } \\ \mathrm{N} / \mathrm{Y}=0 / 1\end{array} & \begin{array}{c}\text { Mobile } \\ \text { Poros. }\end{array} & \begin{array}{c}\text { Immob } \\ \text { Poros }\end{array} & \begin{array}{c}\text { Mass Xfer } \\ \text { Coef }(1 / \mathrm{y})\end{array} & \begin{array}{c}\text { Velocity } \\ (\mathrm{ft} / \mathrm{y})\end{array} \\ 1 & 100 . & 0 . & 0 & 0 . & 0 . & 0 . & 0.1 \\ 2 & 0 . & 0 . & 0 & 0 . & 0 . & 0 & 1.0 \\ 3 & 0 . & 0 . & 0 & 0 . & 0 . & 0 . & 0.5\end{array}$


Table 4.18.

Sample Input File (Continued)

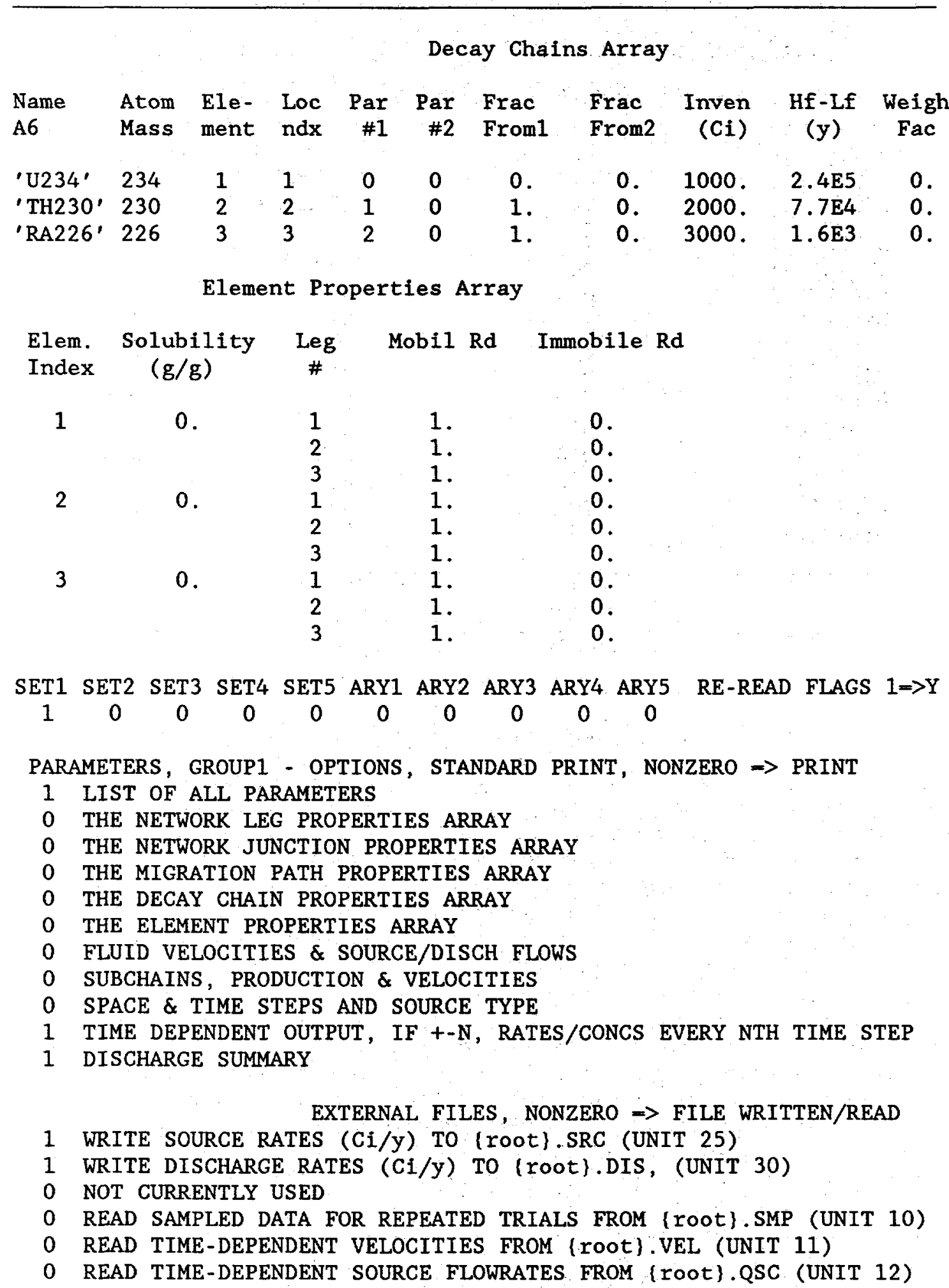


Table 4.18 .

Sample Input File (Concluded)

RUN CONTROLS

0 USE DVM (0) OR ANALYTIC SOLN (NONZERO)

1 SOLVE NETWORK (O) OR INPUT VELOCITIES (NONZERO)

0 . LEACH (0), SOLUBILITIES (1), OR BOTH (2) FOR SOURCE RATES

0 FLOWTHRU (0), MIXCELL (1), OR CHOOSE (2) FOR SOURCE RATES

1 CONSTANT (0) OR EXPONENTIAL (1) LEACH RATE MODEL

0 FORCE (NONZERO) USE OF LEG-TO-LEG TRANSFER ALGORITHM

DEBUG PRINTS, NONZERO $\rightarrow$ PRINT

0 DVM MULTIPLIERS FOR TRANSPORT, DISCHARGE, AND SOURCE

0 DIAGNOSTIC INFO FOR SUBROUTINE BAND

0 DIAGNOSTIC INFO FOR FUNCTION GIT

0 MATRIX/VECTOR SYSTEM SOLVED FOR FLOW

0 THE DATA READ FROM UNIT 10

0 NOT CURRENTLY USED

0 INVENTORY AT RELEASE TIME

0 RESTRICTIONS PLACED ON THE TIME STEPS

0 LEG/JUNCTION \& JUNCTION/LEG CONNECTIONS

0 VELOCITY FIELDS FROM UNIT 11

0 THE ATOM COUNT SUMMARY

0 JUNCTION PRESSURES \& LEG FLOWRATES

0 LEG-TO-LEG TRANSFER FRACTIONS

0 TIME SPENT AS EACH ISOTOPE IN A SUBCHAIN

0 TIME-DEPENDENT SOURCE FLOWRATES

The network for this problem consists of three legs connected end-toend, and the same three legs provide the migration path. Since the flow network is not solved, it is not necessary to provide junction data; that is, the number of junctions is set to zero. One threemember decay chain is transported and the isotopes are of three distinct elements.

Based on the source run control options, the only required entries from the source and flow parameters are the access fraction and the leach rate. Specifically, the source pore volume is not required (the mixing-cell model is not activated); the pore-area of the source regime is not required (neither the mixing cell nor solubilities are treated), the source fluid density is not required (solubilities are not treated), and the pore-area at discharge is not required (concentration output is not requested). 
As indicated by the time parameters, simulation is to occur to 10000 years and leaching and fluid flow both start at closure (i.e., zero years). The time steps for source and transport calculations are to be determined by the code.

Only the inlet and outlet junction numbers and the leg lengths are used from the Network Leg Properties Array. Since diffusion from mobile to immobile fluid is not treated for any of the legs in the migration path, NEFTRAN II transports radionuclides over an average, single-porosity path. Thus, the dispersivity and space step are taken from the first row of the Migration Path Properties Array and the remaining rows are ignored for these two columns. The space step is input as zero which indicates that NEFTRAN II will determine the space increment internally. Average species velocities are found using the fluid velocities provided in the last column and the retardation factors provided in the Element Properties Array. Mobile phase porosities are not required (the network is not solved and diffusion is not treated), immobile phase porosities are not required (diffusion is not treated), and mass-transfer coefficients are not required (diffusion is not treated).

The Decay Chains Array shows the three-member chain transported with corresponding inventories and half-lives. The normalization (weighting) factors are input as zero so the code defaults them to unity. The element numbering scheme must be consistent with the Element Properties Array. If there were additional isotopes of uranium, for example, either in this chain or subsequent decay chains, all should have element index equal to 1 .

The Element Properties Array has NELM $(-3)$ times NPATH $(=3)$ rows. The first row for each element has a different data list than the other rows. Note that each element index in the Decay Chains Array has a corresponding index in the Element Properties Array. Each leg-index in the migration path is repeated for each element. As shown, all elements are not retarded (i.e., $R_{d}=1$ ) for all legs in the migration path. Solubilities are not required (only leaching of waste form is treated) and immobile phase retardations are not required (diffusion is not treated).

With the reading of the ninth row of the Element Properties Array, input of the first data set is complete. NEFTRAN II solves for source and transport then returns to subroutine FLOWIN to check for additional data sets. The second data set is initialized by a descriptive header and flags indicating which data groups are to be re-read. For the second data set, some of the options are disabled, but the change of interest is the choice of leach models. For the second data set, the exponential leach rate model is used to determine source rates instead of the constant leach rate model that was used in the first data set. 
After reading the options, NEFTRAN II performs data checking to help ensure a consistent data set. The code then solves for source and transport using the new options. Following that, it again returns to subroutine FLOWIN to read another data set. At this point, since there is no further input, an end-of-file marker is detected and the program terminates normally. 


\subsection{VERIFICATION AND SAMPLE PROBLEMS}

Three examples of problems simulated with NEFTRAN II are presented in the next three sections. The purpose of these sample problems is to demonstrate the use of different features of the code, as well as to illustrate how NEFTRAN II can be used to simulate different types of problems (e.g., field scale, laboratory scale). The sample problems are also designed to illustrate the process involved in using NEFTRAN II to model real problems since, although NEFTRAN II may appear simple to use, applications of it to real problems require as much thought and analysis as using what might appear to be a more complex code. Each section will include instructions for preparing each input file and each sample problem is also compared with an analytical solution to verify that the computer code has implemented the corresponding mathematical model correctly.

\subsection{Sample Problem 1 - DVM and Analytical Transport Mode1}

The first sample problem is designed to demonstrate the use of the flow network model, the use of both the DVM and analytical transport model, the use of multiple data sets, and the ability of DVM to reproduce the analytical solution output. The problem is based on a hypothetical Reference Site, as was presented by Campbel1 and others (1981b) in the NWFT/DVM user's manual. SWIFT II (Reeves and others, 1986a, 1986b) computer code simulations of the site were used to define the network flow system in NEFTRAN II and to ensure that the network flow model was accurately representing the flow field. The following subsections will present information about the Reference Site, how this information was used to formulate the network flow model in NEFTRAN II, the input required for this problem, and the resultant discharge rate curves for each isotope.

\subsubsection{Reference Site}

The site is located in a symmetrical upland valley, half of which is shown in Figure 5.1. The crest of the ridge surrounding the valley is at an elevation of $6000 \mathrm{ft}$. The crest is a surface water and ground water divide. Therefore, ground-water movement in the valley is from water that has fallen in the valley. The valley is drained by a major river, River L, which is at an elevation of 2500 feet opposite the surface structures of the repository.

The geology of the area near the site is shown in cross-section in Figure 5.2. The valley is underlain by crystalline bedrock which crops out over a narrow strip lying at the ridge crest surrounding the valley. The bedrock is assumed impermeable to ground-water flow. The bedrock is overlain by a sequence of sedimentary rocks as shown in Figure 5.2.

SWIFT II computer code simulations of ground-water flow at the Reference Site were conducted. Figure 5.3 shows the direction of ground-water flow schematically. Flow in the upper sand and gravel aquifer is not shown here as it is hydraulically isolated from the middle sandstone by the 


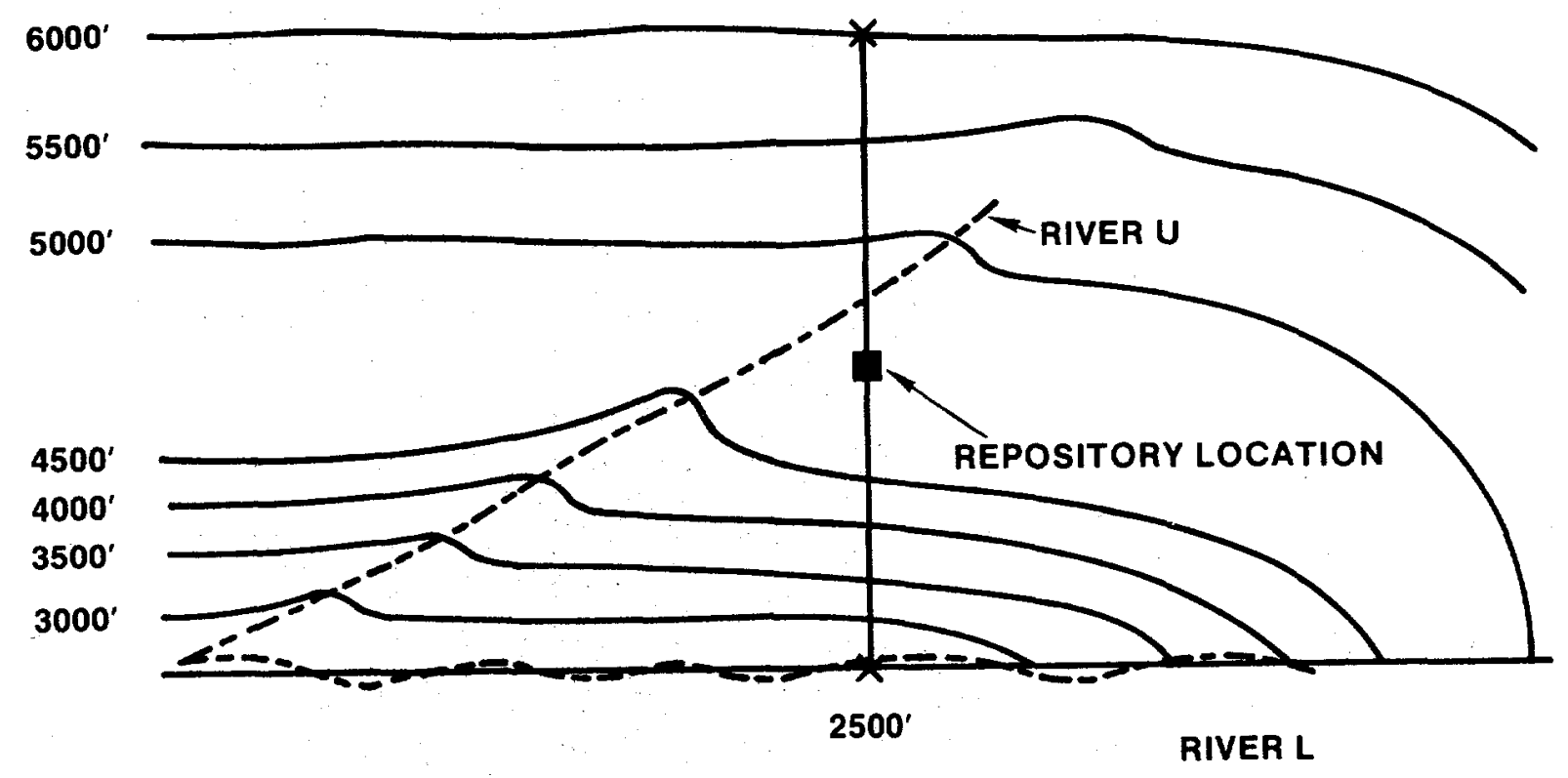

Figure 5.1. Physiographic Setting of the Reference Site 


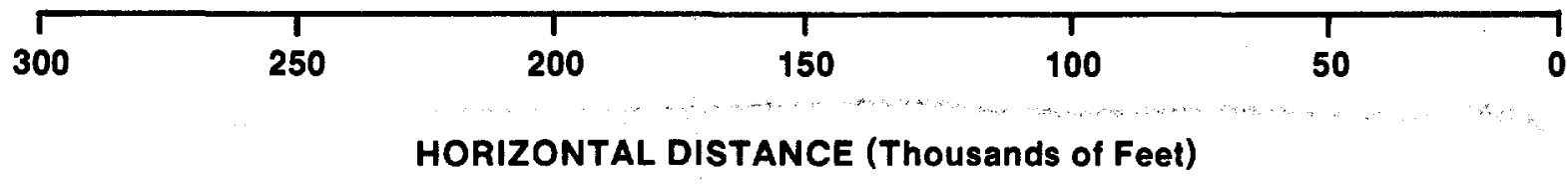

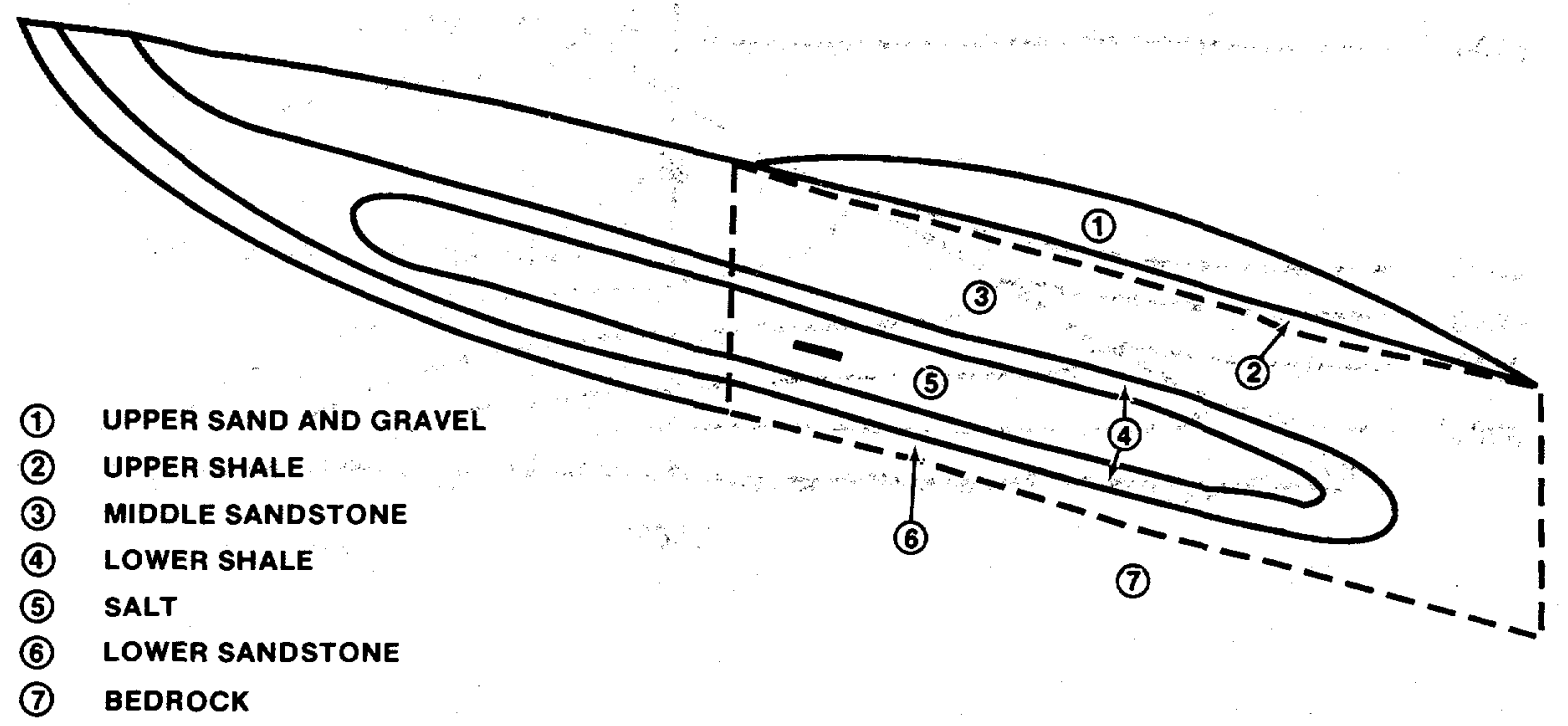

Figure 5.2. Reference Site Geology 


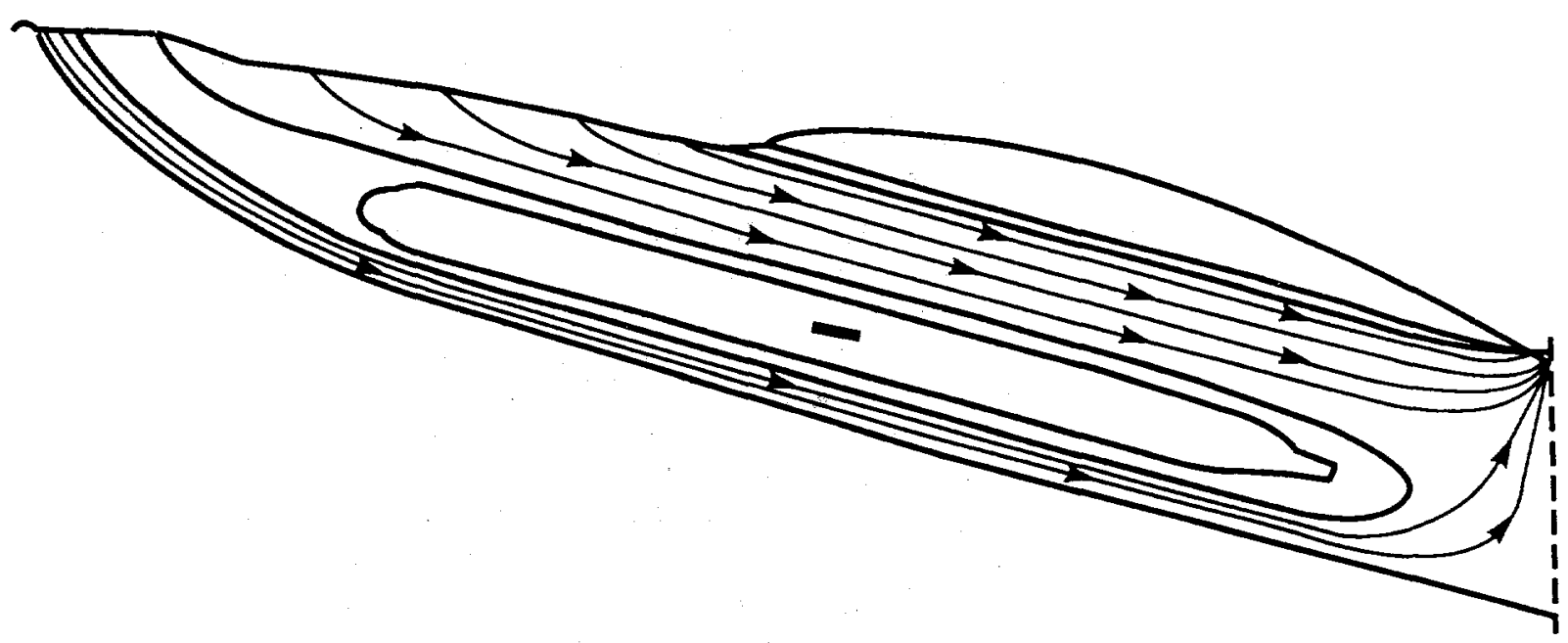

Figure 5.3. Ground-Water Flow at Reference Site 
upper shale. With the exception of disruptive events, the permeability of the shale and salt is sufficiently low that flow through these layers is generally insignificant. A reduced representation of the Reference Site (the outlined portion in Figure 5.2) was used in the SWIFT II computer code simulations to find pressures. Figure 5.4 shows the resultant pressure head distribution and Figure 5.5 shows the gridding used in the SWIFT II simulations.

Both Figures 5.3 and 5.4 suggest that flow in the middle and lower sandstone aquifers (or some portion of them) can be adequately represented by one-dimensional flow systems. Thus, the network flow model contained in NEFTRAN II can be used to reproduce this flow system.

\subsubsection{Network Flow Mode1}

Figure 5.6 shows the NEFTRAN II network superimposed with the darker lines on the Reference Site, and Figure 5.7 shows the NEFTRAN II flow network for this problem with the corresponding leg and junction numbering scheme. Legs $1-4$ of the network are placed at the middle shale/middle sandstone interface and legs 5-8 lie at the lower shale/lower sandstone interface. Legs $9,10,11,12,14$ and 15 are used to represent various disruptive features which affect the salt and shale layers near the repository (leg 13) and connect the repository to the overlying aquifer. The left-hand boundary of the network is taken as the midpoint of Column 29 (Figure 5.6) in order to satisfy two restrictions. First, it is of sufficient distance down-dip from the recharge region to ensure that the fluid flow has an insignificant vertical component. Second, it is far enough up-dip to ensure that most disruptive features near the repository will not affect the pressure boundary conditions. The resulting emplacement of the NEFTRAN II flow network at the Reference Site is critical to the input of boundary pressures, elevations, and leg lengths.

\subsubsection{Data Set \#1}

The input file for this sample problem is given in Table 5.1. Since Chapter 4 gives detailed instructions for data input, only those input parameters that are specific to this sample problem will be discussed in this section. The user is reminded that, the read statements in NEFTRAN II are list-directed; therefore, every parameter and array entry must be assigned a value (i.e., a blank is not permissible).

\section{Options}

As indicated in Table 5.1, the user has requested for several different quantities to be written to the hardcopy output file (\{root\}.OUT) by setting the corresponding option to one. Option 8 and 9 are set to zero indicating that the decay/production and velocities of each subchain, and the space step, time increment and source type will not be written to the output file. The user has also indicated to write the discharge rates to an external file, (root).DIS, in the external file options. 


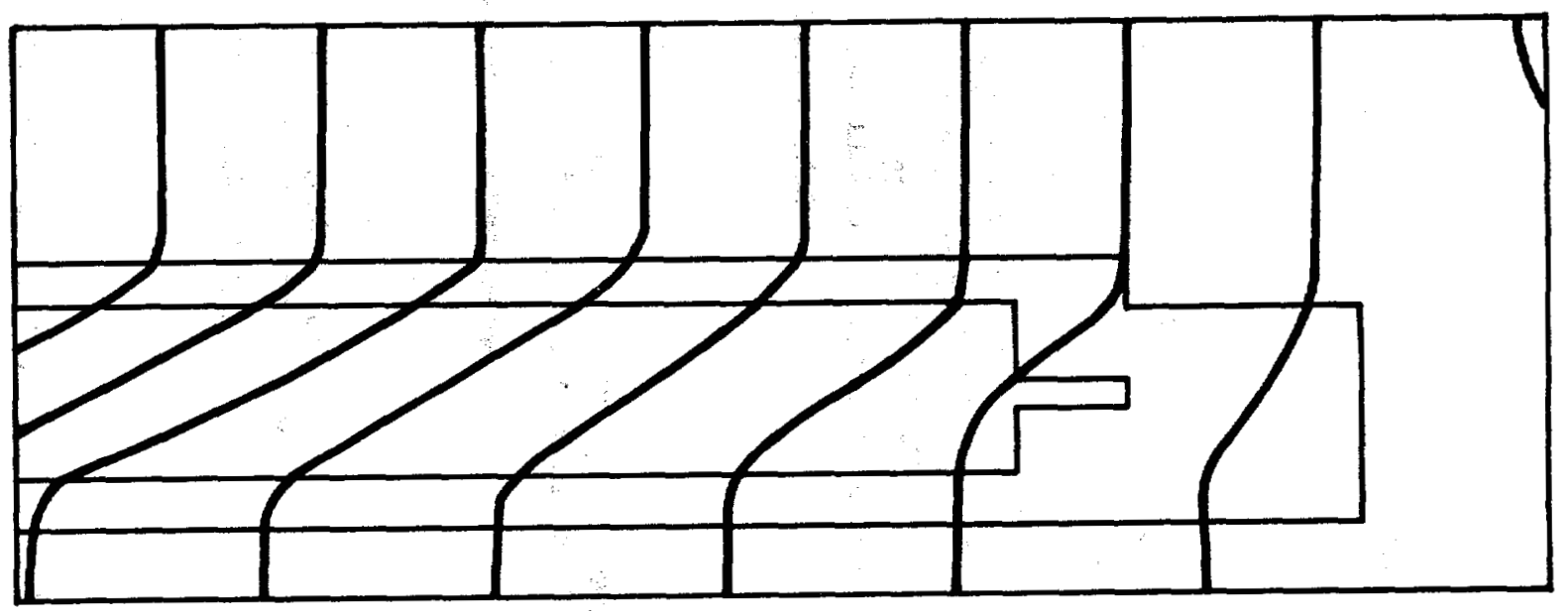

Figure 5.4. Hydraulic Head Distribution at the Reference Site 


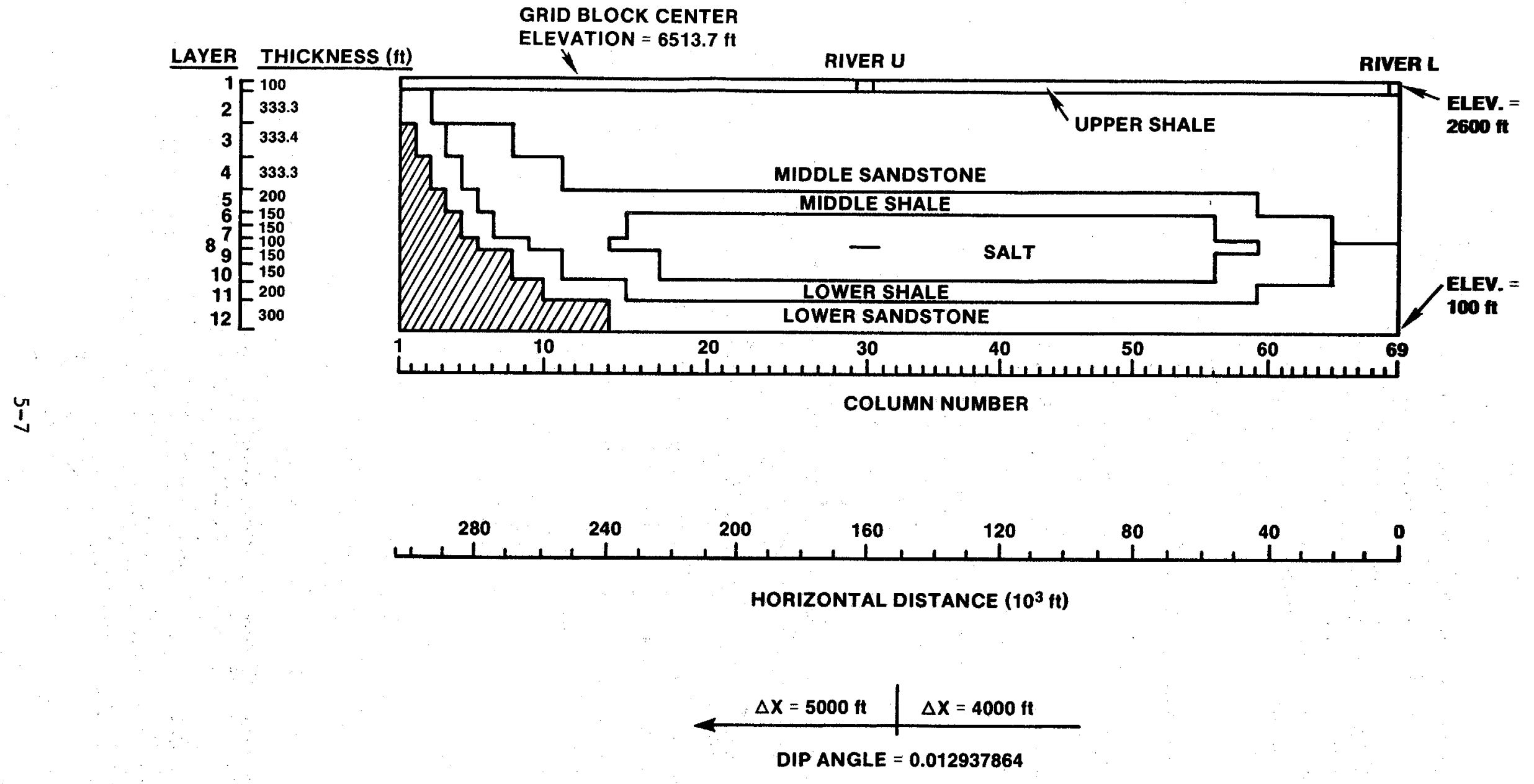

Figure 5.5. SWIFT II Gridding for the Reference Site 

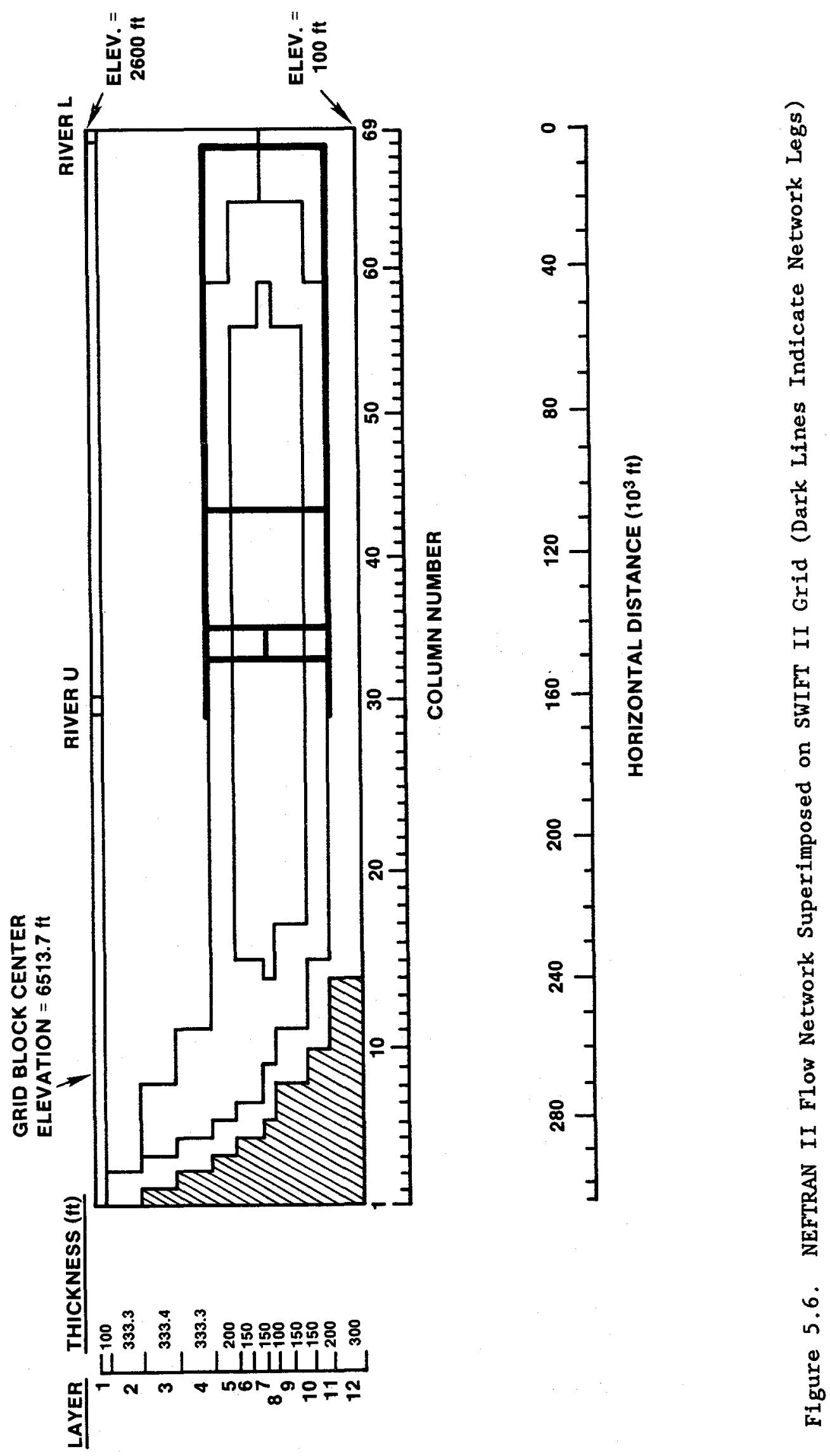


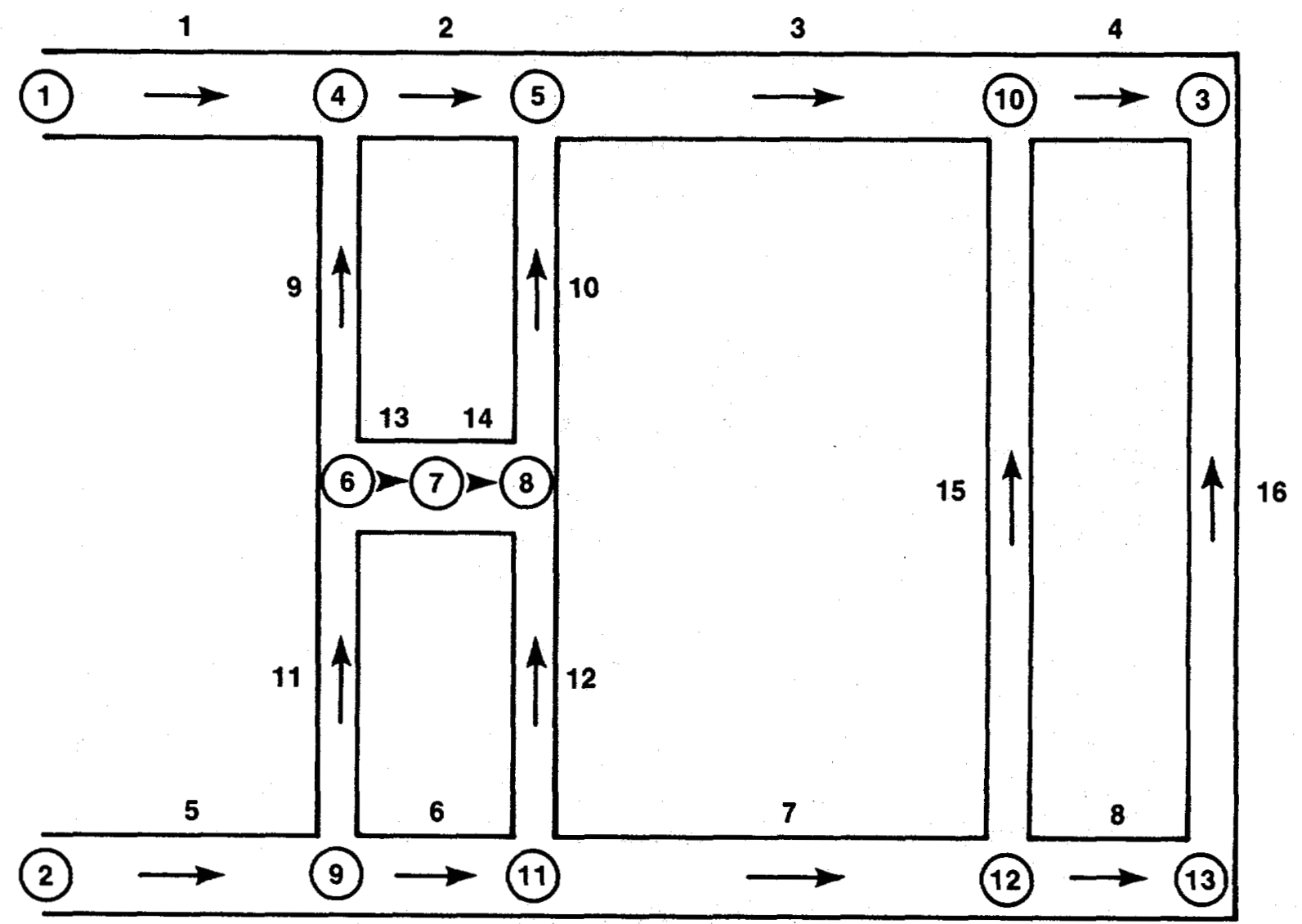

Figure 5.7. NEFTRAN II Flow Network Used in Sample Problem \#1 
Table 5.1.

Input File for Sample Problem \#1

NEFTRAN II: SAMPLE PROBLEM \#1 FOR USER'S MANUAL (PC VERSION)

PARAMETERS, GROUP1 - OPTIONS, STANDARD PRINT, NONZERO $\rightarrow$ PRINT

1 LIST OF ALL PARAMETERS

1 THE NETWORK LEG PROPERTIES ARRAY

1 THE NETWORK JUNCTION PROPERTIES ARRAY

1 THE MIGRATION PATH PROPERTIES ARRAY

1 THE DECAY CHAIN PROPERTIES ARRAY

1 THE ELEMENT PROPERTIES ARRAY

1 FLUID VELOCITIES \& SOURCE/DISCH FLOWS

0 SUBCHAINS, PRODUCTION \& VELOCITIES

0 SPACE \& TIME STEPS AND SOURCE TYPE

1 TIME DEPENDENT OUTPUT, IF $+-N$, RATES/CONCS EVERY NTH TIME STEP

1 DISCHARGE SUMMARY

EXTERNAL FILES, NONZERO $\Rightarrow$ FILE WRITTEN/READ

0 WRITE SOURCE RATES ( $\mathrm{Ci} / \mathrm{y}$ ) TO (root\}. SRC (UNIT 25)

1 WRITE DISCHARGE RATES ( $\mathrm{CI} / \mathrm{y}$ ) TO (root).DIS, (UNIT 30)

0 NOT CURRENTLY USED

0 READ SAMPLED DATA FOR REPEATED TRIALS FROM (root\}.SMP (UNIT 10)

0 READ TIME-DEPENDENT VELOCITIES FROM (root).VEL (UNIT 11)

0 READ TIME-DEPENDENT SOURCE FLOWRATES FROM (root).QSC (UNIT 12)

RUN CONTROLS

1 USE DVM (0) OR ANALYTIC SOLN (NONZERO)

0 SOLVE NETWORK (0) OR INPUT VELOCITIES (NONZERO)

0 LEACH (0), SOLUBILITIES (1), OR BOTH (2) FOR SOURCE RATES

O FLOWTHRU (0), MIXCELL (1), OR CHOOSE (2) FOR SOURCE RATES

0 CONSTANT (0) OR EXPONENTIAL (1) LEACH RATE MODEL

0 FORCE (NONZERO) USE OF LEG-TO-LEG TRANSFER ALGORITHM

$$
\text { DEBUG PRINTS, NONZERO } \Rightarrow \text { PRINT }
$$

0 DVM MULTIPLIERS FOR TRANSPORT, DISCHARGE, AND SOURCE

0 DIAGNOSTIC INFO FOR SUBROUTINE BAND

0 DIAGNOSTIC INFO FOR FUNCTION GIT

O MATRIX/VECTOR SYSTEM SOLVED FOR FLOW

0 THE DATA READ FROM UNIT 10

0 NOT CURRENTLY USED

0 INVENTORY AT RELEASE TIME

0 RESTRICTIONS PLACED ON THE TIME STEPS

1 LEG/JUNCTION \& JUNCTION/LEG CONNECTIONS

0 VELOCITY FIELDS FROM UNIT 11

0 THE ATOM COUNT SUMMARY

1 JUNCTION PRESSURES \& LEG FLOWRATES

0 LEG-TO-LEG TRANSFER FRACTIONS

0 TIME SPENT AS EACH ISOTOPE IN A SUBCHAIN

0 TIME-DEPENDENT SOURCE FLOWRATES 
Table 5.1.

Input File for Sample Problem \#1 (Continued)

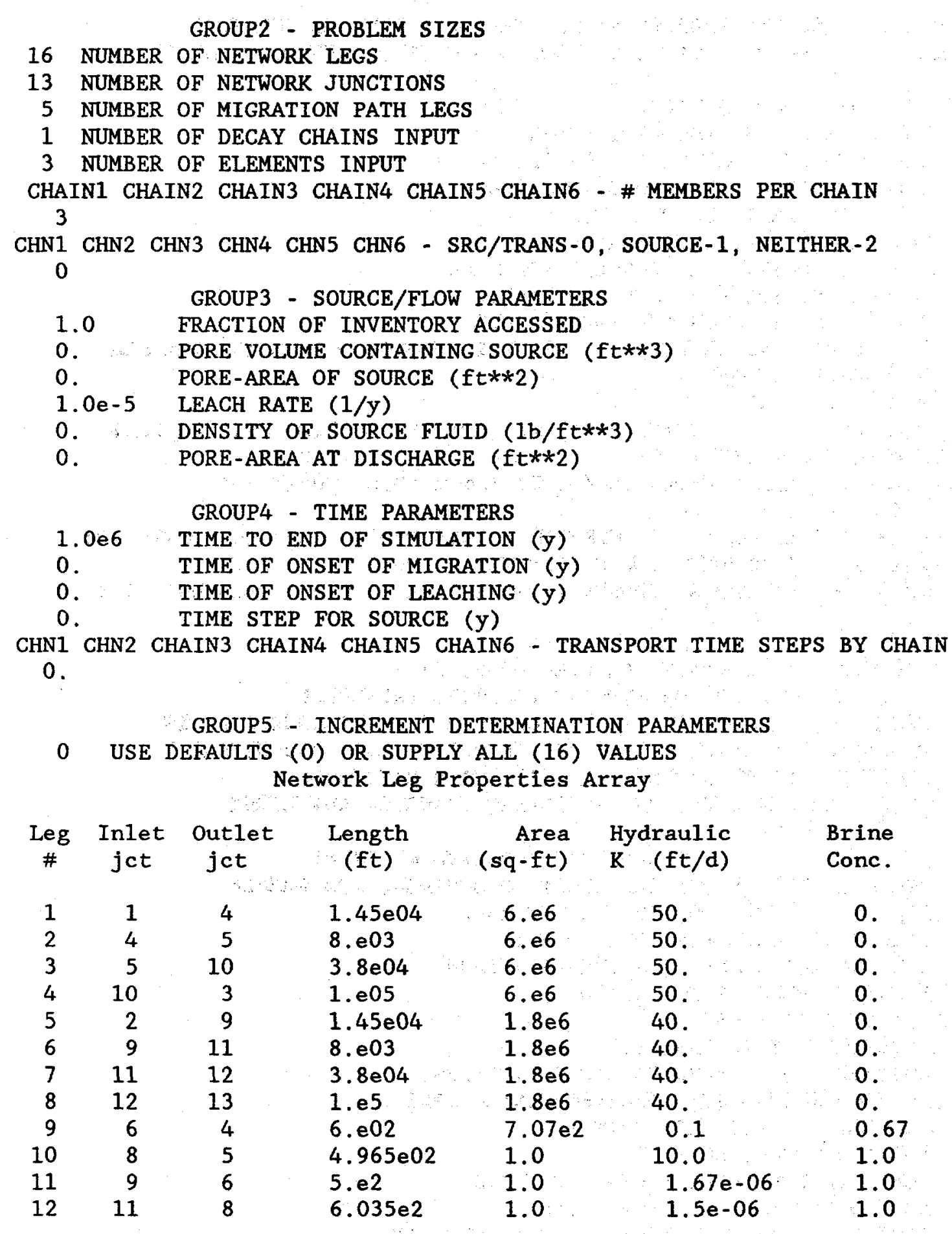


Table 5.1.

Input File for Sample Problem \#1 (Continued)

\begin{tabular}{rrrllll}
\hline 13 & 6 & 7 & $4 . \mathrm{e} 03$ & $5.4 \mathrm{e} 02$ & 10.0 & 1.0 \\
14 & 7 & 8 & $4 . e 03$ & $5.4 \mathrm{e} 02$ & 10.0 & 1.0 \\
15 & 12 & 10 & $1.1 \mathrm{e} 03$ & 1.0 & $1.57 \mathrm{e}-06$ & 1.0 \\
16 & 13 & 3 & $1.1 \mathrm{e} 03$ & $1.2 \mathrm{e} 8$ & 2.5 & 0.
\end{tabular}

Network Junction Properties Array

$\begin{array}{cccc}\begin{array}{c}\text { Jct. } \\ \#\end{array} & \begin{array}{c}\text { Elevation } \\ \text { (ft) }\end{array} & \begin{array}{c}\text { Known Press? } \\ \text { No/Yes }=0 / 1\end{array} & \begin{array}{c}\text { Pressure } \\ \text { (psi) }\end{array} \\ 1 & & 1 & \\ 2 & 3.60241 \mathrm{e} 3 & 1 & 432.7 \\ 3 & 2.50241 \mathrm{e} 3 & 1 & 646.1 \\ 4 & 1.52589 \mathrm{e} 3 & 0 & 432.7 \\ 5 & 3.41481 \mathrm{e} 3 & 0 & 0 . \\ 6 & 3.31131 \mathrm{e} 3 & 0 & 0 . \\ 7 & 2.81481 \mathrm{e} 3 & 0 & 0 . \\ 8 & 2.81481 \mathrm{e} 3 & 0 & 0 . \\ 9 & 2.81481 \mathrm{e} 3 & 0 & 0 . \\ 10 & 2.31481 \mathrm{e3} & 0 & 0 . \\ 11 & 2.81967 \mathrm{e} 3 & 0 & 0 . \\ 12 & 2.21131 \mathrm{e3} & 0 & 0 . \\ 13 & 1.71967 \mathrm{e} 3 & 0 & 0 .\end{array}$

Migration Path Properties Array

$\begin{array}{cccccccc}\begin{array}{c}\text { Leg } \\ \#\end{array} & \begin{array}{c}\text { Disp. } \\ (\mathrm{ft})\end{array} & \begin{array}{c}\text { DX } \\ (\mathrm{ft})\end{array} & \begin{array}{c}\text { Diffus? } \\ \mathrm{N} / \mathrm{Y}=0 / 1\end{array} & \begin{array}{c}\text { Mobile } \\ \text { Poros. }\end{array} & \begin{array}{c}\text { Immob } \\ \text { Poros }\end{array} & \begin{array}{c}\text { Mass Xfer } \\ \text { Coef }(1 / \mathrm{y})\end{array} & \begin{array}{c}\text { Velocity } \\ (\mathrm{ft} / \mathrm{y})\end{array} \\ 13 & 500 . & 0 . & 0 & 0.3 & 0 . & 0 . & 0.0 \\ 14 & 0 . & 0 . & 0 & 0.3 & 0 . & 0 . & 0.0 \\ 10 & 0 . & 0 . & 0 & 0.15 & 0 . & 0 . & 0.0 \\ 3 & 0 . & 0 . & 0 & 0.3 & 0 . & 0 . & 0.0 \\ 4 & 0 . & 0 . & 0 & 0.3 & 0 . & 0 . & 0.0\end{array}$

\begin{tabular}{|c|c|c|c|c|c|c|c|c|c|c|}
\hline & & & & & $\mathrm{Dec}$ & ay Cha & Array & & & \\
\hline $\begin{array}{l}\text { Name } \\
\text { A6 }\end{array}$ & $\begin{array}{l}\text { Atom } \\
\text { Mass }\end{array}$ & $\begin{array}{l}\text { Ele- } \\
\text { ment }\end{array}$ & $\begin{array}{l}\text { Loc } \\
\text { ndx }\end{array}$ & $\begin{array}{r}\text { Par } \\
\# 1\end{array}$ & $\begin{array}{r}\text { Par } \\
\# 2\end{array}$ & $\begin{array}{l}\text { Frac } \\
\text { From1 }\end{array}$ & $\begin{array}{l}\text { Frac } \\
\text { From2 }\end{array}$ & $\begin{array}{c}\text { Inven } \\
(C i)\end{array}$ & $\begin{array}{c}\text { Hf - Lf } \\
(y)\end{array}$ & $\begin{array}{c}\text { Weigh } \\
\text { Fac }\end{array}$ \\
\hline JP237 & 23 & 1 & 1 & 0 & 0 & 0 & 0 & 10 & 2.14 & 1. \\
\hline U233' & 233 & 2 & 2 & 1 & 0 & 1 & 0 . & 1000 . & $1.62 \mathrm{E} 5$ & 1. \\
\hline TH229' & 229 & 3 & 3 & 2 & 0 & 1. & 0. & 1000 . & $7.30 \mathrm{E} 3$ & 1. \\
\hline
\end{tabular}


Table 5.1.

Input File for Sample Problem \#1 (Continued)

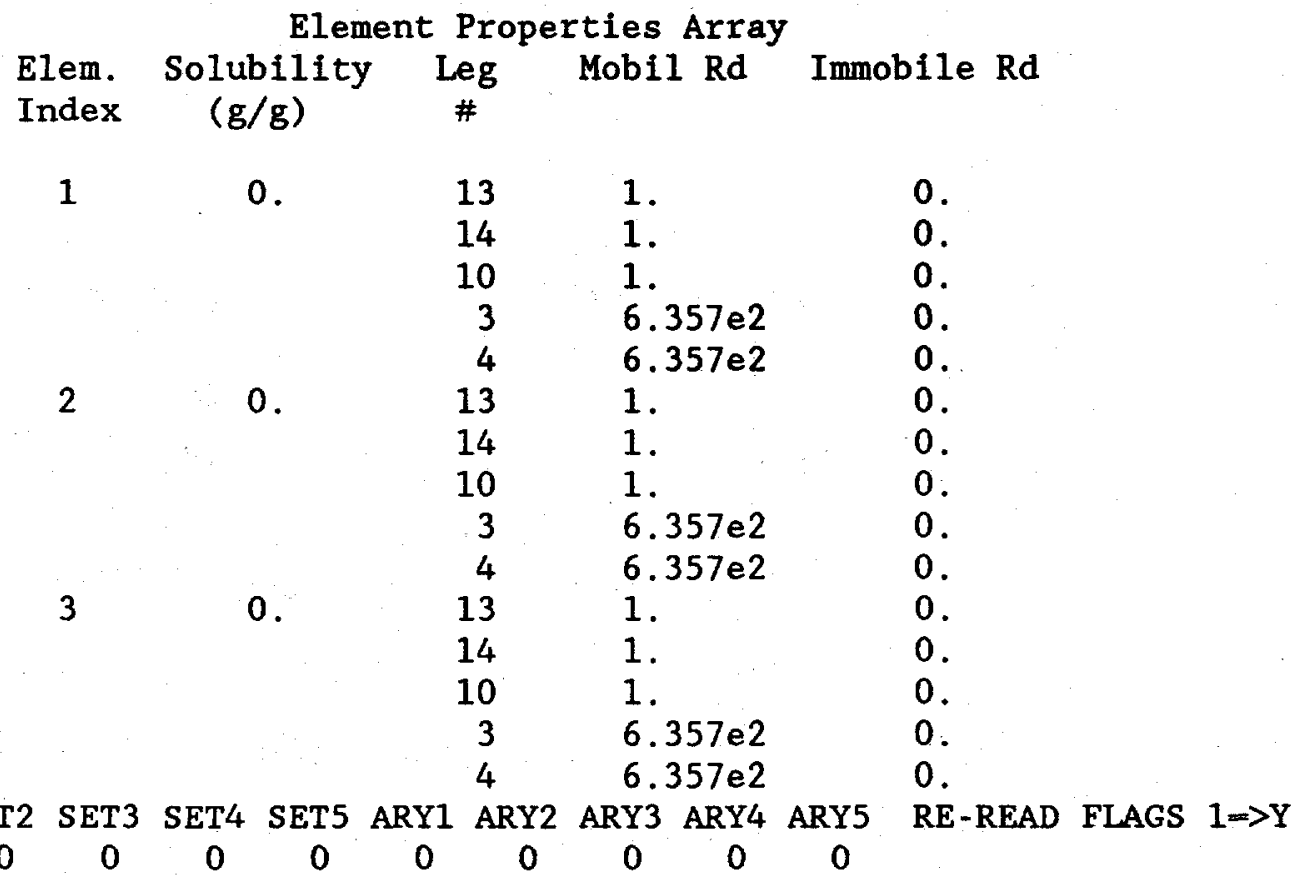

PARAMETERS, GROUP1 - OPTIONS, STANDARD PRINT, NONZERO $\Rightarrow$ PRINT

1 LIST OF ALL PARAMETERS

0 THE NETWORK LEG PROPERTIES ARRAY

0 THE NETWORK JUNCTION PROPERTIES ARRAY

0 THE MIGRATION PATH PROPERTIES ARRAY

0 THE DECAY CHAIN PROPERTIES ARRAY

0 THE ELEMENT PROPERTIES ARRAY

1 FLUID VELOCITIES \& SOURCE/DISCH FLOWS

1 SUBCHAINS, PRODUCTION \& VELOCITIES

1 SPACE \& TIME STEPS AND SOURCE TYPE

1 TIME DEPENDENT OUTPUT, IF $+-N$, RATES/CONCS EVERY NTH TIME STEP

1 DISCHARGE SUMMARY

EXTERNAL FILES, NONZERO $\Rightarrow$ FILE WRITTEN/READ

0 WRITE SOURCE RATES (Ci/y) TO (root).SRC (UNIT 25)

1 WRITE DISCHARGE RATES ( $\mathrm{Ci} / \mathrm{y}$ ) TO \{root\}.DIS, (UNIT 30)

0 NOT CURRENTLY USED

0 READ SAMPLED DATA FOR REPEATED TRIALS FROM (root).SMP (UNIT 10)

0 READ TIME-DEPENDENT VELOCITIES FROM (root).VEL (UNIT 11)

0 READ TIME-DEPENDENT SOURCE FLOWRATES FROM \{root\}.QSC (UNIT 12)

RUN CONTROLS

0 USE DVM (0) OR ANALYTIC SOLN (NONZERO)

0 SOLVE NETWORK (0) OR INPUT VELOCITIES (NONZERO) 
Table 5.1.

Input File for Sample Problem \#1 (Concluded)

0 LEACH (0), SOLUBILITIES (1), OR BOTH (2) FOR SOURCE RATES

0 FLOWTHRU (0), MIXCELL (1), OR CHOOSE (2) FOR SOURCE RATES

0 CONSTANT (0) OR EXPONENTIAL (1) LEACH RATE MODEL

0 FORCE (NONZERO) USE OF LEG-TO-LEG TRANSFER ALGORITHM

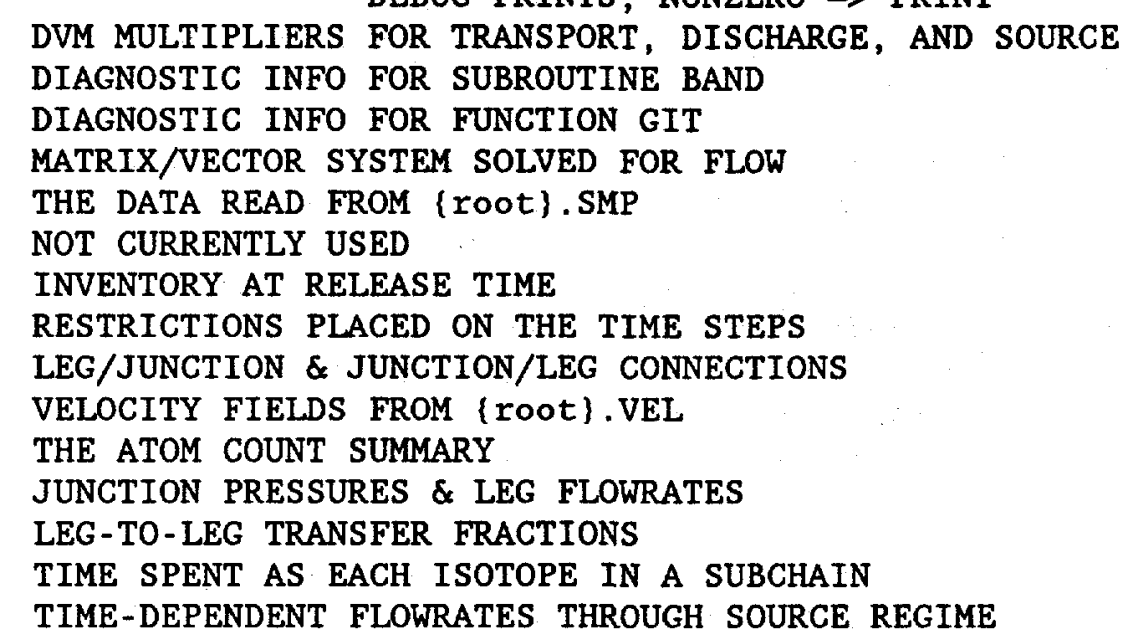

For the first data set, option 18 is set to one to implement the analytical solution transport model contained in NEFTRAN II and not the DVM transport model. This is the option that will change in the second data set, which will be discussed in Section 5.1.4. Option 19 is set to zero which invokes the flow network model. As discussed in Section 2.2, when the analytical solution transport model is being implemented the source model contained in NEFTRAN II is not implemented. Consequently, options 20 and 21 are not applicable and are set to zero, as indicated in Table 5.1. The option to invoke leg-to-leg transfer (option 23) is also not applicable when the analytical transport model is being implemented, and is input as zero.

The next set of options shown in Table 5.1 are the debug print options. The user has requested for the $1 \mathrm{eg} / \mathrm{junction}$ and junction/leg connections, and the junction pressures and leg flow rates to be printed to the hardcopy output file by setting options 32 , and 35 , respectively, to one. At this point all the options that are required on input have been defined.

\section{Parameters}

The first set of parameters required are the problem size parameters. First, the size of the network needs to be defined, and therefore, the 
number of network legs, number of network junctions, and number of migration path legs are needed. As shown in Figure 5.7, there are 16 network legs, and 13 network junctions. The migration path is assumed to occur through legs 13, 14, 10, 3 and 4 ; therefore, 5 legs are in the migration path. This path was chosen arbitrarily for this sample problem. However, in practice, a particle tracker can be used with the flow simulation results to define the migration path in the network.

To finish defining the problem size parameters, the number of decay chains, the number of elements, and the decay chain inclusion indexes are required. As indicated in Table 5.1, the user has input one decay chain and three different elements. The chain inclusion index is not applicable when the analytical transport model is being implemented, and, therefore, is input as zero.

The next group of parameters shown in Table 5.1 is the source/flow parameters. Here, the user has indicated that the complete source be accessed (i.e., the access fraction is equal to one) and that the leach rate is $1.0 \times 10^{-5} \mathrm{y}^{-1}$. In terms of the analytical transport model, the leach time $(\tau)$ for the concentration boundary condition is taken to be the reciprocal of the leach rate. The remaining parameters are not required when the analytical transport model is being implemented and when output concentrations are not desired (option 10 is positive), and are input as zero.

As shown in Table 5.1, for the time parameters, the user has indicated that the simulation time is one million years, and that release and leaching begin at time zero. The source and transport time steps and the increment-determination parameters are not applicable when the analytical solution is implemented and therefore zero is input for these parameters:

\section{Network Leg Properties Array}

Table 5.1 presents the Network Leg Properties Array. The first three columns of this array are easily input based on the resultant NEFTRAN II flow network of the Reference site shown in Figure 5.7. Column four requires the length of each leg. These lengths can be determined from Figure 5.6. Specifically, from the right edge of the repository to River $\mathrm{L}$ midpoint is $138000 \mathrm{ft}$ (length of $\mathrm{leg} 3+\mathrm{leg} 4$ ). Repository length is $8000 \mathrm{ft}$ (length of $\mathrm{leg} 13+\mathrm{leg} 14$ ) and is assumed to be level. Leg 14 is assumed to be part of the repository, but not to contain any waste; therefore, leg 13 is designated the source leg for transport and assumed to be $4000 \mathrm{ft}$. From the left edge of the repository to the aquifer inlets in the flow network is $14500 \mathrm{ft}$ (length of leg 1 and leg 5). Vertical legs must maintain a total length of $1100 \mathrm{ft}$ (length of leg $9+$ leg $11, \operatorname{leg} 10+\operatorname{leg} 12, \operatorname{leg} 15$ and $\operatorname{leg} 16)$. Leg 15 is arbitrarily placed $138000 \mathrm{ft}$ (length of leg 7 and leg 8) down dip from legs 10 and 12 .

Column five in the Network Leg Properties Array requires the crosssectional area of each leg. For the Reference Site, the aquifer widths are assumed to be that of the repository $(6000 \mathrm{ft})$. Upper and lower aquifer depths (1000 ft and $300 \mathrm{ft}$, respectively) can be found from 
Figure 5.6 as well as the width of $1 \mathrm{eg} 16$ (20000 ft). Based on this data the cross-sectional areas of each $\mathrm{leg}$ are given by:

$$
\begin{array}{ll}
(\text { legs } 1-4) & 1000 \times 6000=6 \times 10^{6} \mathrm{ft}^{2}, \\
(\text { legs } 5-8) & 300 \times 6000=1.8 \times 10^{6} \mathrm{ft}^{2}, \\
(\operatorname{leg~16)} & 20000 \times 6000=1.2 \times 10^{8} \mathrm{ft}^{2}
\end{array}
$$

The rest of the legs were assigned arbitrary cross-sectional areas since these legs represent disruptive events that may effect the flow system.

The next required input for the Network Leg Properties Array is the hydraulic conductivity of each leg (data column 6). The Reference Site hydraulic properties used in the SWIFT II calculations are shown in Table 5.2. Appropriate hydraulic conductivities for legs $1,2,3$, and 4 are the horizontal conductivity of the middle sandstone. The horizontal conductivity of the lower sandstone is appropriate for legs 5, 6, 7, and 8. The horizontal conductivity of the repository area ( $\operatorname{leg} 13$ and $\operatorname{leg}$ 14) is assumed to be the five orders of magnitude higher than the conductivity of salt. As the hydraulic conductivity across the salt and shale is essentially vertical, an effective vertical conductivity of salt and shale to legs $9,10,11,12$, and 15 is assigned. Similarly, leg 16 represents vertical flow through portions of both the middle and lower sandstone. The differing media can be viewed as providing resistance to flow in series and therefore, one effective conductivity for the differing media can be found. For example, the effective conductivity of leg 16 is given by:

$$
R_{16}=R_{L}+R_{M}
$$

where $R_{16}$ is the total resistance to be assigned leg $16, R_{L}$ the resistance of lower sandstone, $R_{M}$ the resistance of middle sandstone,

and:

$$
R_{i}=\frac{L_{i}}{K_{1} A_{i}}
$$

where $L_{i}$ is the length ( $\left.f t\right), K_{i}$ is the hydraulic conductivity (ft/day), $A_{1}$ is the cross-sectional area $\left(\mathrm{ft}^{2}\right)$.

Substituting the appropriate values for each media type into Equation 5.4 and rearranging Equation 5.5, the effective conductivity of 1 eg 16 can be found.

The last column of input data needed for the Network Leg Properties Array is the brine concentration (C) of each leg. The interior legs are assumed to contain saturated brine $(C=1)$, except for leg 9 (assumed $C=$ $0.67)$, and the aquifer legs are assumed to be fresh water $(C=0)$. 
Table 5.2 .

Reference Site Hydraulic Properties

\begin{tabular}{lccc}
\hline & $\begin{array}{c}\text { Horizontal Hydraulic } \\
\text { Conductivity } \\
\text { (ft/day) }\end{array}$ & $\begin{array}{c}\text { Vertical Hydraulic } \\
\text { Conductivity } \\
\text { (ft/day) }\end{array}$ & Porosity \\
\cline { 2 - 3 } $\begin{array}{l}\text { Middle } \\
\text { Sandstone }\end{array}$ & 50 & 1.4 & 0.3 \\
Lower Shale & $10^{-2}$ & $10^{-3}$ & 0.3 \\
Salt & $10^{-5}$ & $10^{-6}$ & 0.03 \\
$\begin{array}{l}\text { Lower } \\
\text { Sandstone }\end{array}$ & 40 & 7.0 & 0.3 \\
& 4 & & \\
\hline
\end{tabular}

\section{Network Junction Properties Array}

The next input array shown in Table 5.1 is the Network Junction Properties Array. The first column is a listing of each junction in ascending order. The second column requires the elevation of each junction. To find the elevations the SWIFT II simulations are used. SWIFT II flow simulations accounted for the dip angle at the Reference Site. Using the sine of this dip angle and the top center of the SWIFT II $y$-axis grid blocks as measuring points, the grid block distance vertically (i.e., y = 0 to $y=29$ ) is $140000 \mathrm{ft}$. Since the elevation of the first grid block is known to be $6514 \mathrm{ft}$, the elevation of the bottom grid block is given by:

$$
6514 \mathrm{ft}-140000 \mathrm{ft} * \text { sine (dip angle) }=4702 \mathrm{ft} \text {. }
$$

The elevation of junction 1 (the upper aquifer inlet) is therefore,

$$
4702.41 \mathrm{ft}-1100 \mathrm{ft}=3602 \mathrm{ft} .
$$

With this reference point, the leg lengths and dip angle are used to determine the remaining junction elevations.

The next column in the Network Junction Properties Array is the flag that indicates whether the pressure is known at that boundary. For this flow network, pressures at elevation for junctions 1,2 and 3 are required boundary conditions for the network flow model. Therefore, the flag is set to 1 for junctions 1,2 , and 3 in the third column of the Junction Properties Array. 
Next, in the Network Junction Properties Array is the actual pressure values for the boundary junctions (In this case, Junctions 1,2 and 3 ). These pressures are taken from the output of the SWIFT II simulation of the Reference Site. SWIFT II calculates pressures at the top center of each grid block. Junctions 1 and 2 correspond directly to grid blocks in the SWIFT II simulation. Junction 3 is treated somewhat differently. The SWIFT II output indicates that pressures along the elevation of Junction 3 show little change from columns $29-65$ with a value of approximately 433 psi (see Figure 5.5). However, from columns $66-69$ pressures increase in order to drive the flow upward to River $L$. The discharge point in NEFTRAN II is $1100 \mathrm{ft}$ below River L; consequently, there is no need for NEFTRAN II to simulate the pressure increase indicated by SWIFT II. Therefore, the pressure at Junction 3 is assumed to be approximately 433 psi. NEFTRAN II flow calculations are more similar to those of SWIFT II when the input outlet pressure is assumed to be constant and not to increase.

\section{Migration Path Properties Array}

The next array shown in Table 5.1, is the Migration Path Properties Array. The first column in this array is the leg number for each leg in the migration path. These leg numbers are in reference to the flow network and, as defined arbitrarily, are legs 13, 14, 10, 3 and 4 . The next column in this array requires a dispersivity for each leg. When the analytical solution is being implemented, the only dispersivity that is utilized is the one input for the first leg. As Table 5.1 indicates, a dispersivity of $500 \mathrm{ft}$ is assumed for this problem. The next column in the Migration Path Properties Array is the space step column which is not applicable when the analytical solution is being implemented. Implementation of the matrix diffusion model is also not relevant for the analytical solution transport model and, therefore, data input for the fourth, sixth, and seventh columns of this array are not pertinent and are input as zero.

The column in this array that does requires input data is the mobile phase porosity column. The porosity of legs 3 and 4 are taken from the values for middle sandstone in Table 5.2. The repository, legs 13 and 14, is assigned a porosity one order of magnitude higher than salt, and the leg 10 porosity is assumed to be 0.15 . For the last column in this array, zeroes have been input, since the network flow model has been implemented and the user does not need to input pore velocities.

\section{Decay Chain Array}

The next array to be presented in Table 5.1 is the Decay Chain Array. The decay chain that is being transported for this sample problem is ${ }^{237} \mathrm{~Np} \rightarrow{ }^{233} \mathrm{U} \rightarrow{ }^{229} \mathrm{Th}$. For each of these three isotopes, the user has supplied: 1) an isotopic name, 2) the atomic mass of the isotope, 3) an element index, 4) a local decay chain index, 5) the local index of parent number 1 and 2,6 ) the fraction from parent number 1 and 2,7 ) the inventory, 8) the half-life and 9) the weighting factor. For this chain, ${ }^{233} \mathrm{U}$ and ${ }^{229} \mathrm{Th}$ each have one parent and each parent decays completely into the daughter (i.e., the fraction from parent number 1 is one). The user 
has input $1000 \mathrm{Ci}$ initial inventory and a weighting factor equal to one for each isotope.

\section{Element Properties Array}

The last array to be read in the input file shown in Table 5.1 , is the Element Properties Array. The first column is the element index that is equivalent to the element index defined in the Decay Chain Array. The solubilities and the immobile retardation factors required for this array are input as zero since this data is not relevant for the analytical solution implementation. For the mobile phase retardation factors, the only legs that retard any of the isotopes are legs 3 and 4 , and for all three isotopes, the retardation factor in these legs is input as 635 . Implementation of the analytical solution transport model requires that the retardation factors are equal for all isotopes.

\subsubsection{Data Set \#2}

The next input shown in Table 5.1 is the array (INP) that indicates which input data group will be changed for the next data set. As indicated in Table 5.1 , the only data input that will be modified for the next data set is SET1, the options. For the first group of options, the standard print options, the user has indicated that the input arrays will not be written to the output file by setting the corresponding options to zero. The user has requested other information to be printed to the output file by setting the corresponding options to one.

The next set of options that are different from the first data set are the run options. Here, the most significant change between the data sets is accomplished by implementing the DVM transport model instead of the analytical transport model (1.e., setting option 18 to zero instead of one). As discussed in Section 2.3, when the DVM transport model is being implemented, the source model contained in NEFTRAN II is utilized. Consequently, options 20 and 21 are relevant for the second data set. In Table 5.1, option 20 equal to zero indicates that only leaching will be modeled in determining DVM source rates, and option 21 equal to zero indicates that the flow-through source model will be used to distribute the source into the source leg (see Section 2.4.8). Option 23 is input as zero which indicates that the leg-to-leg transfer model will not be implemented for DVM transport. Therefore, DVM transport will be simulated using leg-averaged properties (see Section 2.4). Finally, the debug print options are changed so that no debug information is written to the output file for the second data set. The rest of the required input for this second data set is taken from the data input in the first data set.

In the first data set the user input a zero for some data entries to indicate that these data were not relevant for the analytical transport mode1. By changing the run option to the DVM transport model, the zeroes that were input in the first data set may have different meanings when used in the second data set. For example, the zeroes that were input for the source time step and transport time step indicate that the code is to determine these time steps internally for the DVM transport model. Other examples can be found in the Migration Path Array (e.g., space step) and the Element Properties Array (e.g., solubility limit). 


\section{1 .5 Results}

An example of the output file for this sample problem can be found in Appendix E. As requested by the user on input, the first information written to the output file is the input quantities. The user should note that warnings are written to the output file if data inconsistencies are found but are not fatal. This occurs when data is input but is not used (i.e., if dispersivities would have been input for each leg). The remainder of the output file contains the output information the the user has requested.

Figure 5.8, Figure 5.9 and Figure 5.10 show the resultant discharge rate curves for ${ }^{237} \mathrm{~Np},{ }^{233} \mathrm{U}$, and ${ }^{229} \mathrm{Th}$, respectively. Each figure contains the results obtained utilizing the analytical transport model and the DVM transport model contained in NEFTRAN II. Agreement between the two solutions, for each isotope is quite good and indicates that, for this problem, the DVM transport model reproduces results obtained by an analytical solution. Therefore, the code has implemented the mathematical model (the convective-dispersion equation) correctly.

\subsection{Sample Problem 2 - Matrix Diffusion}

The purpose of this sample problem is to demonstrate the matrix diffusion model capability in NEFTRAN II, and by comparing results to an analytical solution, to verify that the matrix diffusion model contained in NEFTRAN II has implemented the mathematical model correctly. This problem also illustrates the process of equating input variables required by the analytical solution to NEFTRAN II input variables.

As discussed in section 2.4.10, the conceptual model for the matrix diffusion model contained in NEFTRAN II consists of assuming that two phases are present in the transport system, a mobile and an immobile phase. The immobile phase serves as a diffusive sink/source for solutes that are being transported in the mobile phase. This conceptual model could be used to represent transport through connected/dead-end pores, liquid/vapor, and fractures/matrix systems. An analytical solution for this conceptual model is given by van Genuchten and Wierenga (1976) and is used for comparison in this sample problem.

The input required for the analytical solution is taken from van Genuchten and Wierenga (1976) and is listed as follows:

$$
\begin{aligned}
& \mathrm{q}=\text { volumetric flow velocity }=10 \mathrm{~cm} / \text { day } \\
& D=\text { dispersion coefficient }=30 \mathrm{~cm}^{2} / \text { day } \\
& \rho=\text { bulk density }=1.30 \mathrm{~g} / \mathrm{cm}^{3} \\
& L=\text { length of column }=30 \mathrm{~cm} \\
& f=\text { fraction adsorption sites in mobile region }=0.40
\end{aligned}
$$




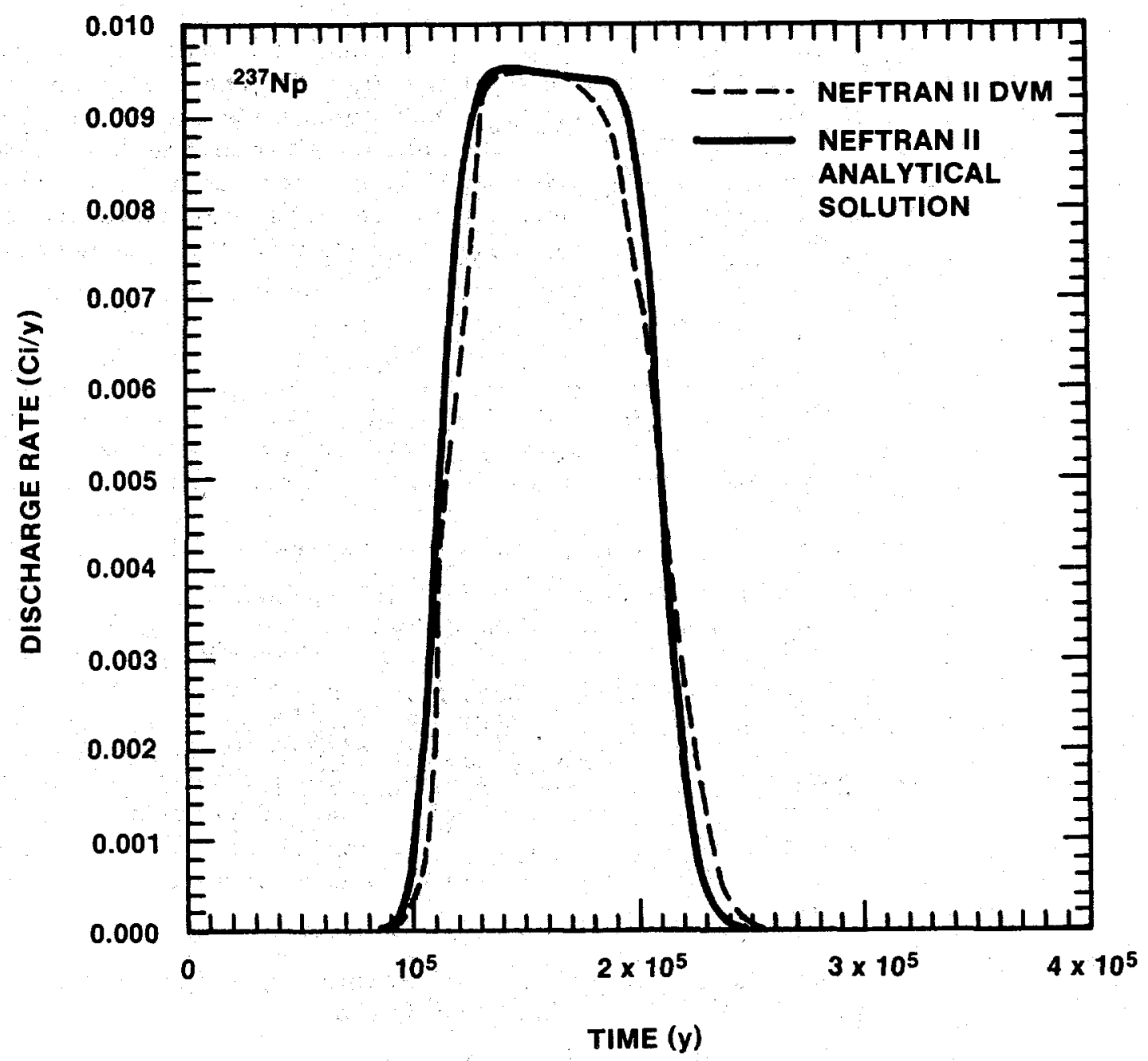

Figure 5.8, ${ }^{237} \mathrm{~Np}$ Discharge Rates for Sample Problem \#1 


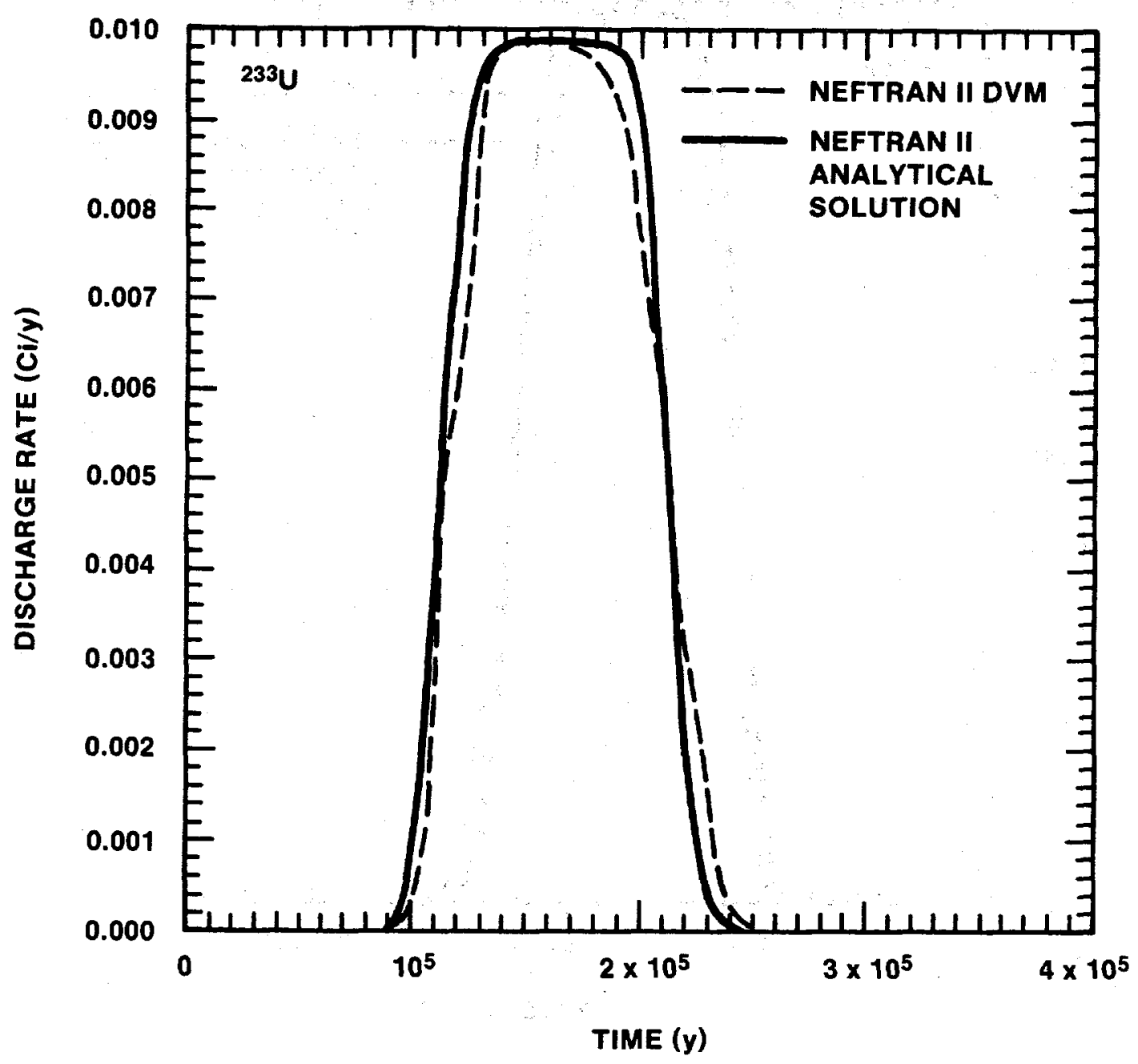

Figure 5.9. 233U Discharge Rates for Sample Problem \#1 


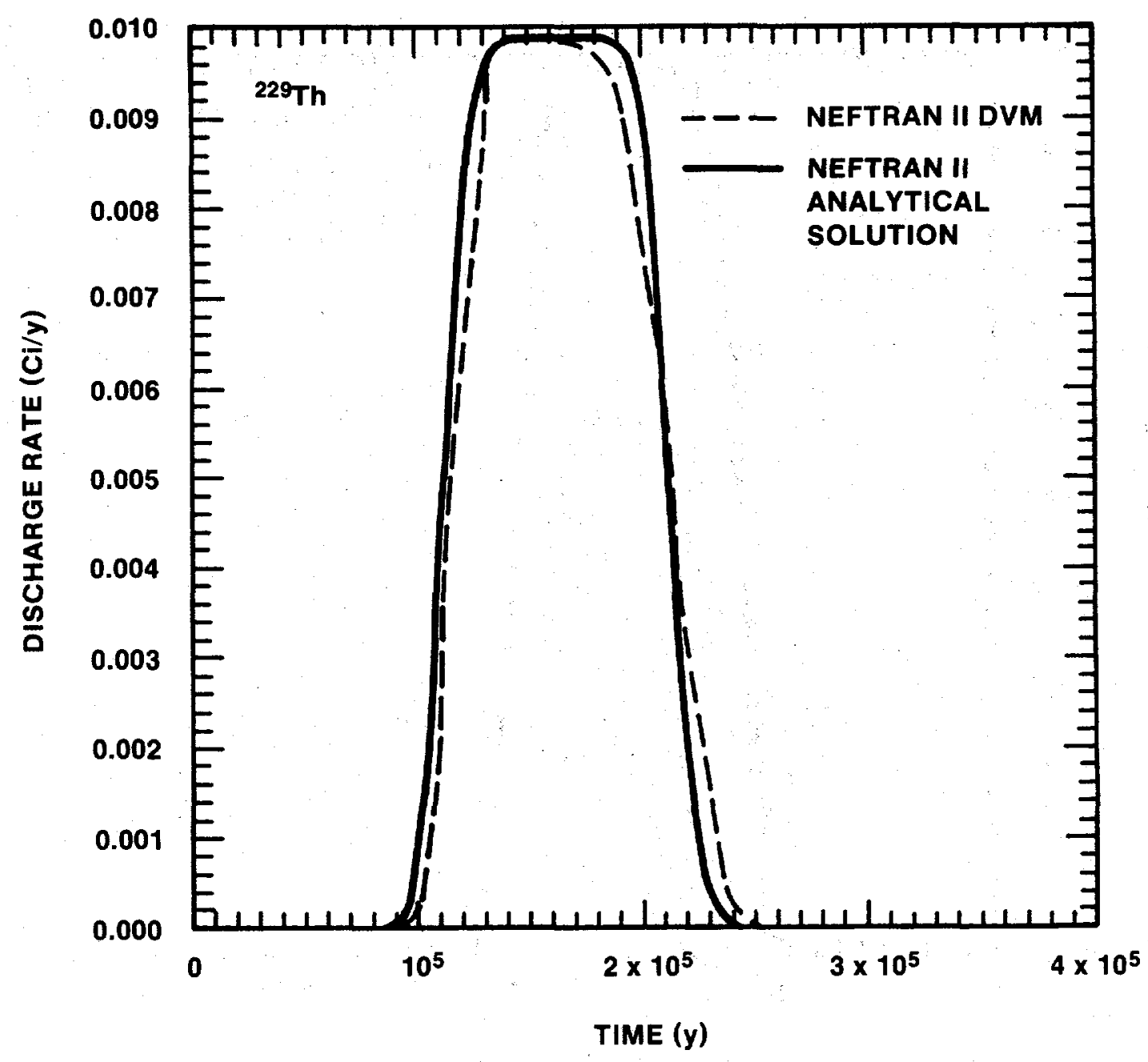

Figure 5.10. ${ }^{229} \mathrm{Th}$ Discharge Rates for Sample Problem \#1 


$$
\begin{aligned}
& \alpha=\text { mass transfer coefficient }=0.15 \text { day }-1 \\
& \mathrm{~T}_{1}=\text { dimensionless pulse period }-3.0 \\
& \mathrm{~K}=\text { distribution coefficient }=0.5 \mathrm{~cm}^{3} / \mathrm{g} \\
& \phi=\text { fraction mobile water }=0.65 \\
& \theta=\text { water content }-0.40 \\
& \theta_{\mathrm{m}}=\phi \theta=\text { mobile phase water content }=0.26 \\
& \theta_{1 \mathrm{~m}}=\theta-\theta_{\mathrm{m}}-\text { immobile phase water content }-0.14
\end{aligned}
$$

Before these data can be input into a NEFTRAN II input file, several conversions must be performed to determine the equivalent NEFTRAN II input variables. Each variable given above, and the equivalent NEFTRAN II required input is as follows:

\section{1. water content $\rightarrow$ porosities}

The mobile and immobile water contents given above are equivalent to the mobile and immobile porosities required by NEFTRAN II.

\section{2. adsorption data $\rightarrow$ retardation factors}

Utilizing the fraction of adsorption sites in the mobile phase, the bulk density and the distribution coefficient given above, the mobile $\left(R_{m}\right)$ and immobile phase $\left(R_{\text {Im }}\right)$ retardations required by NEFTRAN II are

$$
R_{m}=1+\frac{f \rho K}{\theta_{m}},
$$

and

$$
R_{i m}=1+\frac{(1-f) \rho K}{\theta_{i m}},
$$

respectively.

3. volumetric fluid velocity $\rightarrow$ pore velocity

In NEFTRAN II, assuming a unit cross-sectional area, the volumetric fluid velocity given above is equivalent to the Darcy velocity. Therefore, the pore velocity through the migration path is

$$
v_{m}=\frac{q}{\theta_{m}}
$$


which must be converted from $\mathrm{cm} /$ day to $\mathrm{ft} / \mathrm{yr}$ for input into NEFTRAN II. By inputting the pore velocity directly into NEFTRAN II, the network flow model is not used and the Network Junctions Property Array does not need to be input (set number of junctions equal to zero).

Also, due to the unit cross-sectional area, the fluid volumetric flow rate (Q) through the source, that will be needed to determine source parameters subsequently, is equivalent to $q$.

4. dispersion coefficient + dispersivity

NEFTRAN II requires input of the dispersivity based on

$$
D-\alpha \mathrm{v} .
$$

Therefore, using the dispersion coefficient given above, the corresponding dispersivity $\alpha$ can be found from

$$
\alpha=\frac{D}{v_{m}}
$$

where $\alpha$ in centimeters must be converted to feet for input into NEFTRAN II.

5. mass transfer coefficient $\rightarrow$ mass exchange coefficient

The mass transfer coefficient given above is equivalent to the mass exchange coefficient used in NEFTRAN II except for units (day ${ }^{-1}$ must be converted to $\mathrm{y}^{-1}$ ).

6. length of column $\rightarrow$ migration path

Since the network flow model is not being implemented, the flow network is structured only with regard to the migration path. NEFTRAN II requires a minimum of two legs in the migration path, where the first leg acts as a source leg. Consequently, the network is structured to contain two legs which is equivalent to the two legs in the migration path. The only input needed for the Network Properties Array is the length of the first and second leg. The length of the column given above and the length of the second leg in the migration path are equivalent except for the units (centimeters must be converted to feet). The length of the source leg is assumed to be 108 of the length of the second leg. This is based on making the first leg as small as possible since it is not physically representing any part of the column, but not too small that the DVM model (e.g., time or space step criteria) is effected.

7. no decay $\rightarrow$ half-1ife

The van Genuchten and Wierenga (1976) analytical solution does not include radioactive decay. To accomplish this in NEFTRAN II, the halflife of the input solute should be set relatively high compared to the simulation time, to ensure that decay of the solute does not occur during the simulation. 
8. pulse time $\rightarrow$ leach rate

To approximate the constant flux boundary condition for the analytical solution (see van Genuchten and Wierenga), the constant leach-limited only, flow-through source model should be implemented in NEFTRAN II, by inputting the appropriate run options. Consequently, one of the required source input parameters is the leach rate. The pulse time given above is in dimensionless pore volume units. This is equivalent to

$$
T_{1}=\frac{v_{m^{\phi}}}{L t_{1}}
$$

where $t_{1}$ is the leach time (in days). Solving for this leach time and taking the reciprocal is the constant leach rate required by NEFTRAN II (days must be converted to years).

\section{9. unit source concentration $\rightarrow$ initial inventory}

Part of the boundary condition for the analytical solution is a constant unit source concentration throughout the pulse period. Requiring a unit source concentration is equivalent in NEFTRAN II to requiring that the source rate $S(\mathrm{Ci} / \mathrm{yr})$ be equal to the volumetric flow rate $Q$ ( $\left.\mathrm{ft}^{3} / \mathrm{y}\right)$ throughout the leach time, with no decay. In other words, $S$ divided by $Q$ must equal 1. In NEFTRAN II the source rate for a leach limited only source model is given by

$$
s=\frac{c_{0}}{t_{1}}
$$

where $C_{0}$ is the initial inventory. Therefore, substituting $S$ equal to $Q$, the initial inventory is

$$
C_{0}=Q t_{1}
$$

Recal1 that $Q$ is equivalent to $q$ given above, assuming a unit crosssectional area (convert from $\mathrm{cm} /$ day to $\mathrm{ft} / \mathrm{y}$ ).

Applying the above conversions, the resultant NEFTRAN II input file is shown in Table 5.3 and the output file is shown in Appendix $E$. Because the objective of this problem was to compare the NEFTRAN II results to the analytical solution results, the resultant NEFTRAN II discharge rates for the solute are converted to dimensionless times and relative concentrations. Figure 5.11 shows the breakthrough curve for both the analytical solution and the NEFTRAN II simulation. NEFTRAN II reproduces the analytical solution quite well verifying that, for this data set, the matrix diffusion model in NEFTRAN II is a correct representation of the mathematical governing equations presented by van Genuchten and Wierenga (1976). 
Table 5.3.

Input File for Sample Problem \#2

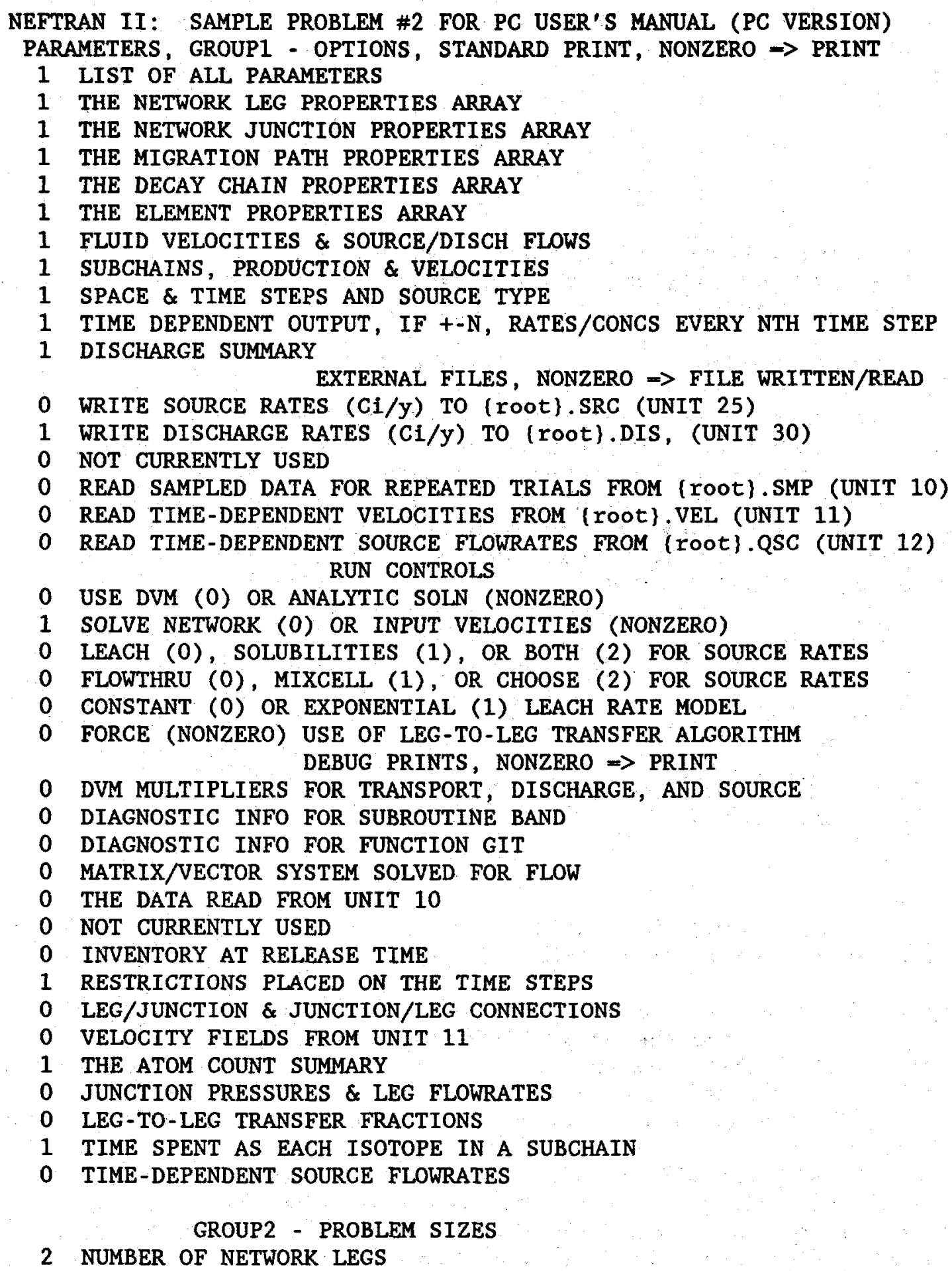


Table 5.3.

Input File for Sample Problem \#2 (Continued)

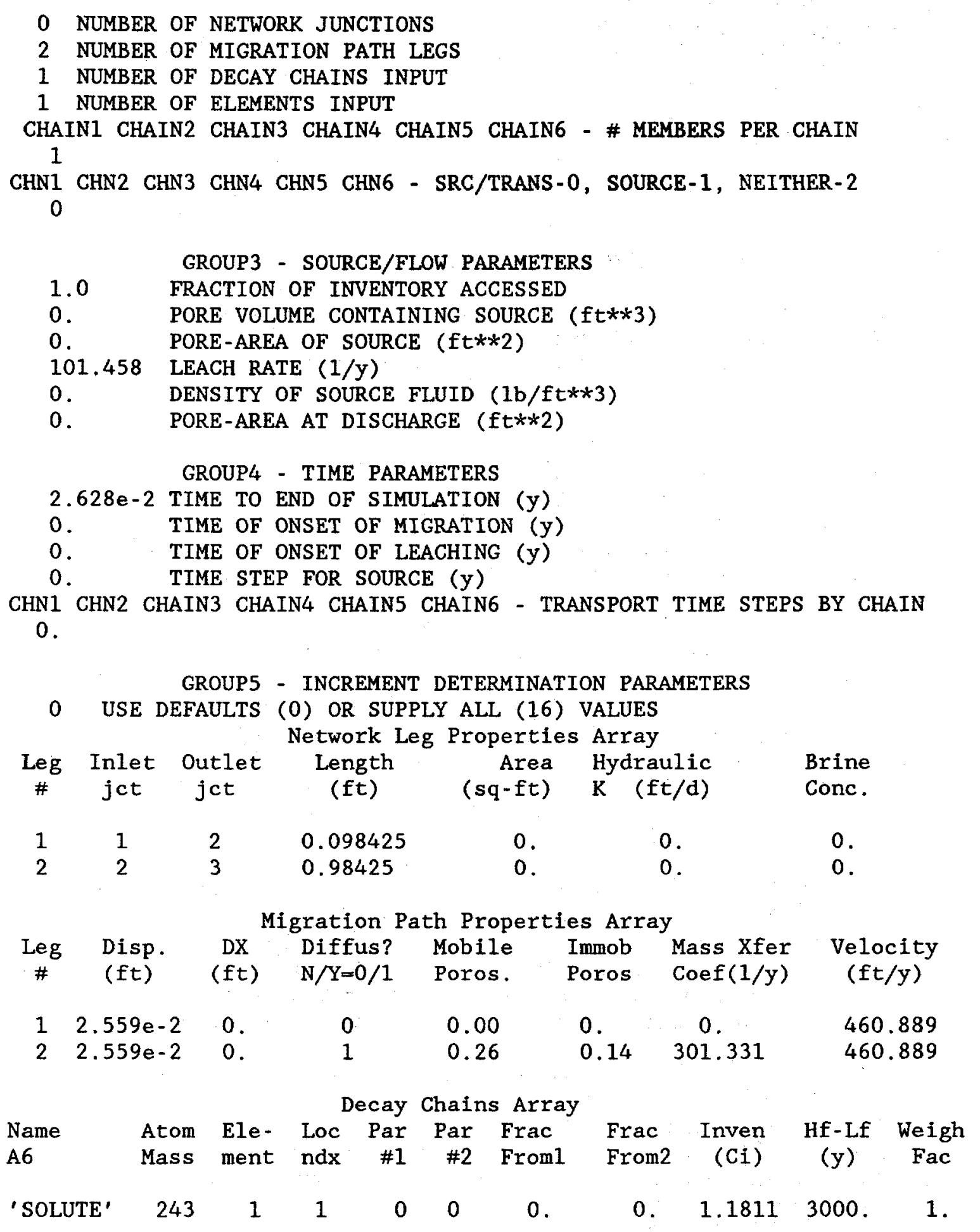


Table 5.3.

Input File for Sample Problem \#2 (Concluded)

\begin{tabular}{ccccc}
\multicolumn{5}{c}{ Element Properties Array } \\
Elem. & Solubility & Leg & Mobil Rd & Immobile Rd \\
Index & $(\mathrm{g} / \mathrm{g})$ & $\#$ & & \\
& & 1 & 1.0 & 0. \\
1 & 0 & 1 & 2.0 & 3.786
\end{tabular}

\subsection{Sample Problem 3 - Time-dependent DVM Velocities}

The purpose of this sample problem is to demonstrate the ability of NEFTRAN II to transport radionuclides in a time-varying flow field and to verify that the DVM transport model is implemented correctly for timedependent velocity conditions.

The analytical solution that is used for the comparison is given by Gelbard (1989). The solution is developed for arbitrary time-dependent fluid-flow velocities and dispersion coefficients. However, the solution is constrained to radionuclides that have identical retardation factors. The boundary and initial condition for the analytical solution are an instantaneous release of radionuclides at time zero from a region $-h \leq x$ $\leq 0$.

Table 5.4 shows the NEFTRAN II input file for this sample problem and Table 5.5 shows the external file that contains the time-varying velocity data. The output file for this sample problem is shown in Appendix $E$. An attempt was made to use input parameters that may be representative of a HLW repository located in unsaturated, fractured media. This is indicated in the following discussion by including the reference for the data that was used. However, the results should not be considered as indicative of the performance of such a repository.

As shown in Table 5.4, the simulation time for this problem is 80000 years. As indicated in Table 5.5, initially, a constant fluid-flow velocity of $3.0 \mathrm{~cm} / \mathrm{yr}$ (Travis and others, 1984) is assumed. At 50000 years the fluid-flow velocity is doubled. The basic underlying assumption for this problem is that the flow direction does not change with time, but only the magnitude of the velocity is time-dependent.

The migration path length is taken to be $200 \mathrm{~m}$ (Travis and others, 1984), which is equivalent to the length of the second leg. The length of the first leg in the migration path, the source leg, is assumed to be 108 of the length of the second leg. This is done because the analytical solution does not account for transport in the source regime, and therefore, the first leg should be as short as possible without affecting the DVM transport model. The dispersivity for each leg is assumed to be $10 \%$ of the length of the leg. 


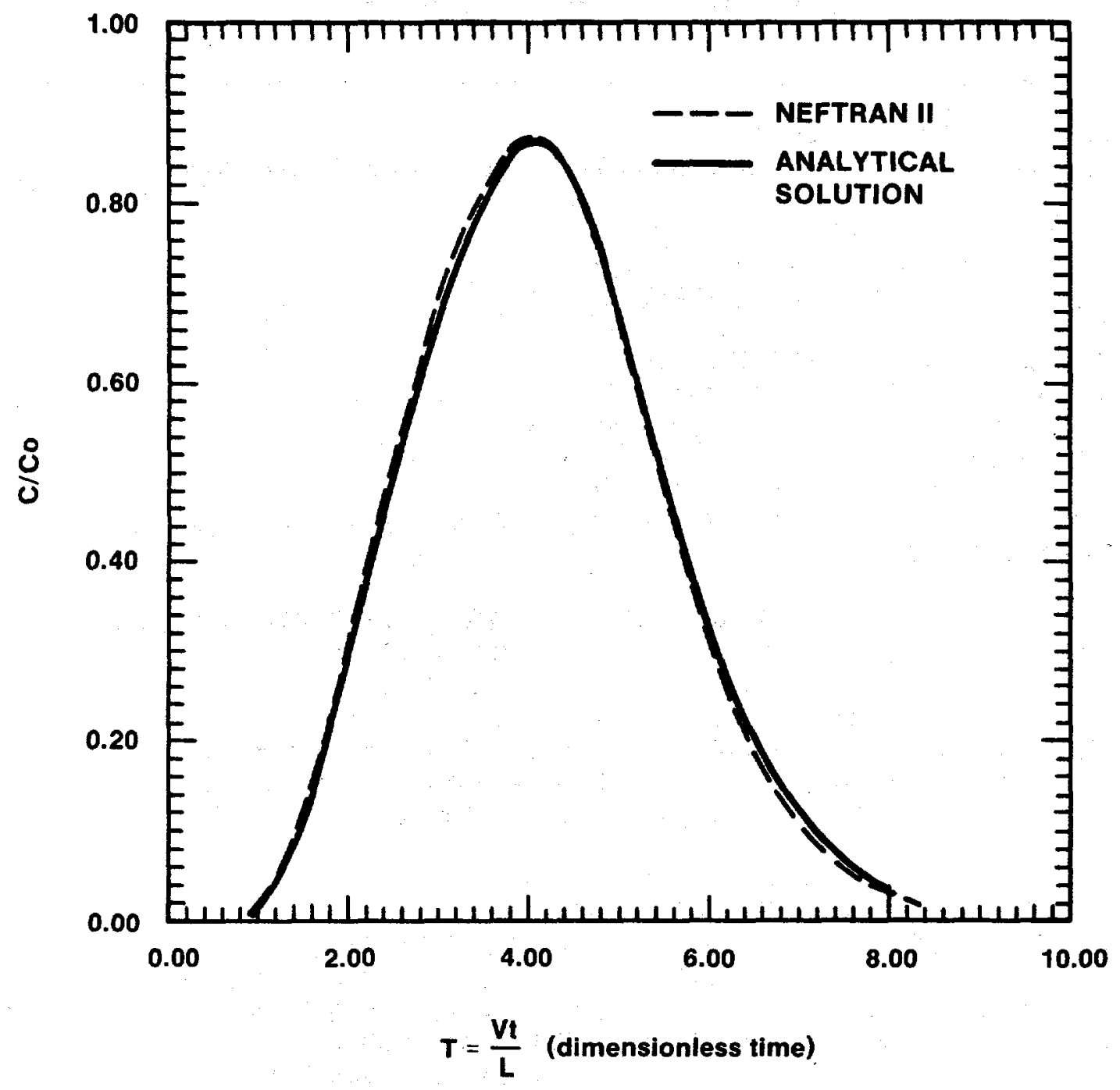

Figure 5.11. Breakthrough Curve for Sample Problem \#2 
Transport is simulated for the three member chain of ${ }^{243} \mathrm{Am},{ }^{239} \mathrm{Pu}$ and ${ }^{235 \mathrm{U}}$, with half-lives of 7593 years, 24400 years and $7.1 \times 10^{8}$ years, respectively, and initial inventories of $6.0 \times 10^{5} \mathrm{Ci}, 1.3 \times 10^{7} \mathrm{Ci}$, and $7.5 \times 10^{2} \mathrm{Ci}$, respectively. These inventories correspond to the amount of HLW that may be placed in a HLW repository (Sinnock and others, 1984). A retardation factor of 19 is assumed for all the radionuclides.

Table 5.4 .

Input File for Sample Problem \#3

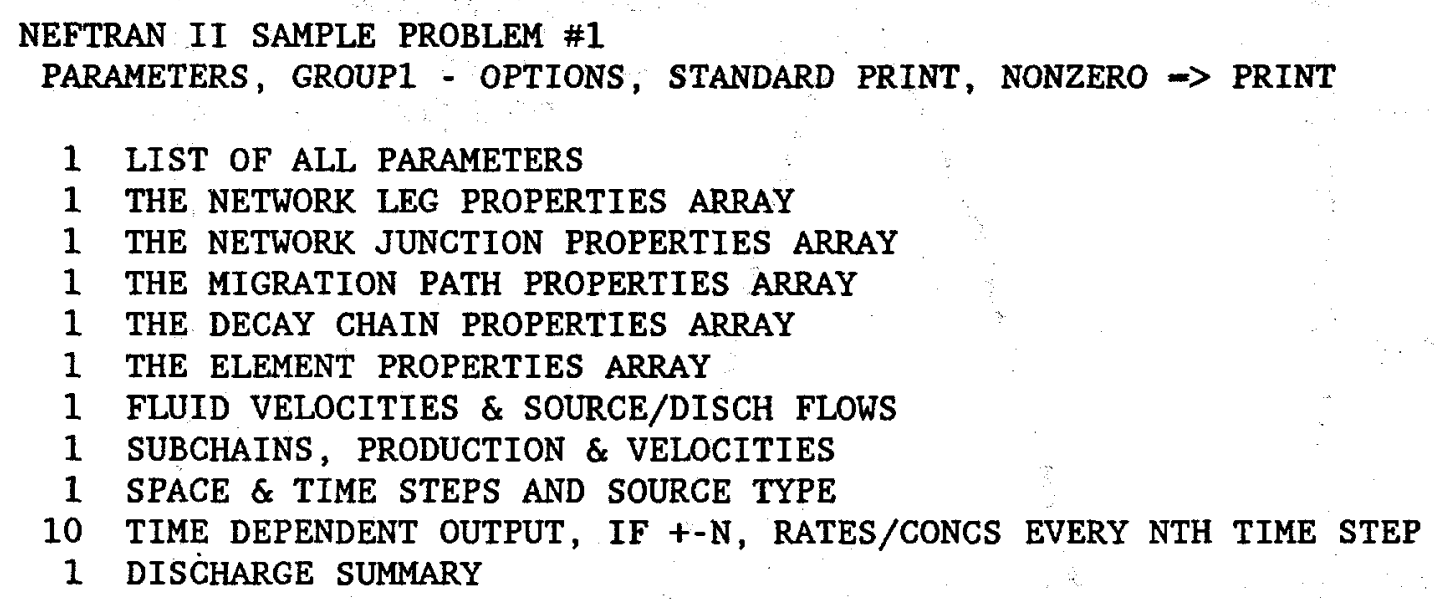

EXTERNAL FILES, NONZERO $\Rightarrow$ FILE WRITTEN/READ

1 WRITE SOURCE RATES (Ci/Y) TO (root).SRC (UNIT 25)

1 WRITE DISCHARGE RATES ( $\mathrm{Ci} / \mathrm{y}$ ) TO \{root\}.DIS, (UNIT 30)

0 NOT CURRENTLY USED

0 READ SAMPLED DATA FOR REPEATED TRIALS FROM \{root\}.SMP (UNIT 10)

1 READ TIME-DEPENDENT VELOCITIES FROM \{root\}.VEL (UNIT 11)

0 READ TIME-DEPENDENT SOURCE FLOWRATES FROM \{root\}.QSC (UNIT 12)

RUN CONTROLS

O USE DVM (0) OR ANALYTIC SOLN (NONZERO)

1 SOLVE NETWORK (0) OR INPUT VELOCITIES (NONZERO)

0 LEACH (0), SOLUBILITIES (1), OR BOTH (2) FOR SOURCE RATES

O FLOWTHRU (0), MIXCELL (1), OR CHOOSE (2) FOR SOURCE RATES

0 CONSTANT (0) OR EXPONENTIAL (1) LEACH RATE MODEL

0 FORCE (NONZERO) USE OF LEG-TO-LEG TRANSFER ALGORITHM

DEBUG PRINTS, NONZERO $\Rightarrow$ PRINT

0 DVM MULTIPLIERS FOR TRANSPORT, DISCHARGE, AND SOURCE

0 DIAGNOSTIC INFO FOR SUBROUTINE BAND

0 DIAGNOSTIC INFO FOR FUNCTION GIT

0 MATRIX/VECTOR SYSTEM SOLVED FOR FLOW

0 THE DATA READ FROM UNIT 10

0 NOT CURRENTLY USED

0 INVENTORY AT RELEASE TIME 
Table 5.4.

Input File for Sample Problem \#3 (Continued)

1 RESTRICTIONS PLACED ON THE TIME STEPS

0 LEG/JUNCTION \& JUNCTION/LEG CONNECTIONS

1 VELOCITY FIELDS FROM UNIT 11

0 THE ATOM COUNT SUMMARY

0 JUNCTION PRESSURES \& LEG FLOWRATES

0 LEG-TO-LEG TRANSFER FRACTIONS

0 TIME SPENT AS EACH ISOTOPE IN A SUBCHAIN

0 TIME-DEPENDENT SOURCE FLOWRATES

GROUP2 - PROBLEM SIZES

2 NUMBER OF NETWORK LEGS

O NUMBER OF NETWORK JUNCTIONS

2 NUMBER OF MIGRATION PATH LEGS

1 NUMBER OF DECAY CHAINS INPUT

3 NUMBER OF ELEMENTS INPUT

CHAIN1 CHAIN2 CHAIN3 CHAIN4 CHAIN5 CHAIN6 - \# MEMBERS PER CHAIN 3

CHN1 CHN2 CHN3 CHN4 CHN5 CHN6 - SRC/TRANS-0, SOURCE-1, NEITHER-2

0

GROUP3 - SOURCE/FLOW PARAMETERS

FRACTION OF INVENTORY ACCESSED

0 . PORE VOLUME CONTAINING SOURCE ( $\mathrm{ft} * * 3$ )

0 . PORE - AREA OF SOURCE ( $f t * * 2)$

1.0e-4 LEACH RATE $(1 / \mathrm{y})$

0 . DENSITY OF SOURCE FLUID (1b/ft**3)

0 . PORE-AREA AT DISCHARGE ( $\mathrm{ft} * * 2)$

GROUP4 - TIME PARAMETERS

8.0e4 TIME TO END OF SIMULATION (y)

0 . TIME OF ONSET OF MIGRATION $(y)$

0 . TIME OF ONSET OF LEACHING ( $y$ )

1000.0 TIME STEP FOR SOURCE (y)

CHN1 CHN2 CHAIN3 CHAIN4 CHAIN5 CHAIN6 - TRANSPORT TIME STEPS BY CHAIN 1000.0

GROUP5 - INCREMENT DETERMINATION PARAMETERS

0 USE DEFAULTS (0) OR SUPPLY ALL (16) VALUES

Network Leg Properties Array

$\begin{array}{ccccrcc}\text { Leg } & \text { Inlet } & \text { Outlet } & \text { Length } & \text { Area } & \text { Hydraulic } & \text { Brine } \\ \# & j c t & j c t & (f t) & (s q-f t) & K(f t / d) & \text { Conc. }\end{array}$

$\begin{array}{ccccccc}1 & 1 & 2 & 1.5 & 0 . & 0 . & 0 . \\ 2 & 2 & 3 & 656 . & 0 . & 0 . & 0 .\end{array}$


Table 5.4.

Input File for Sample Problem \#3 (Concluded)

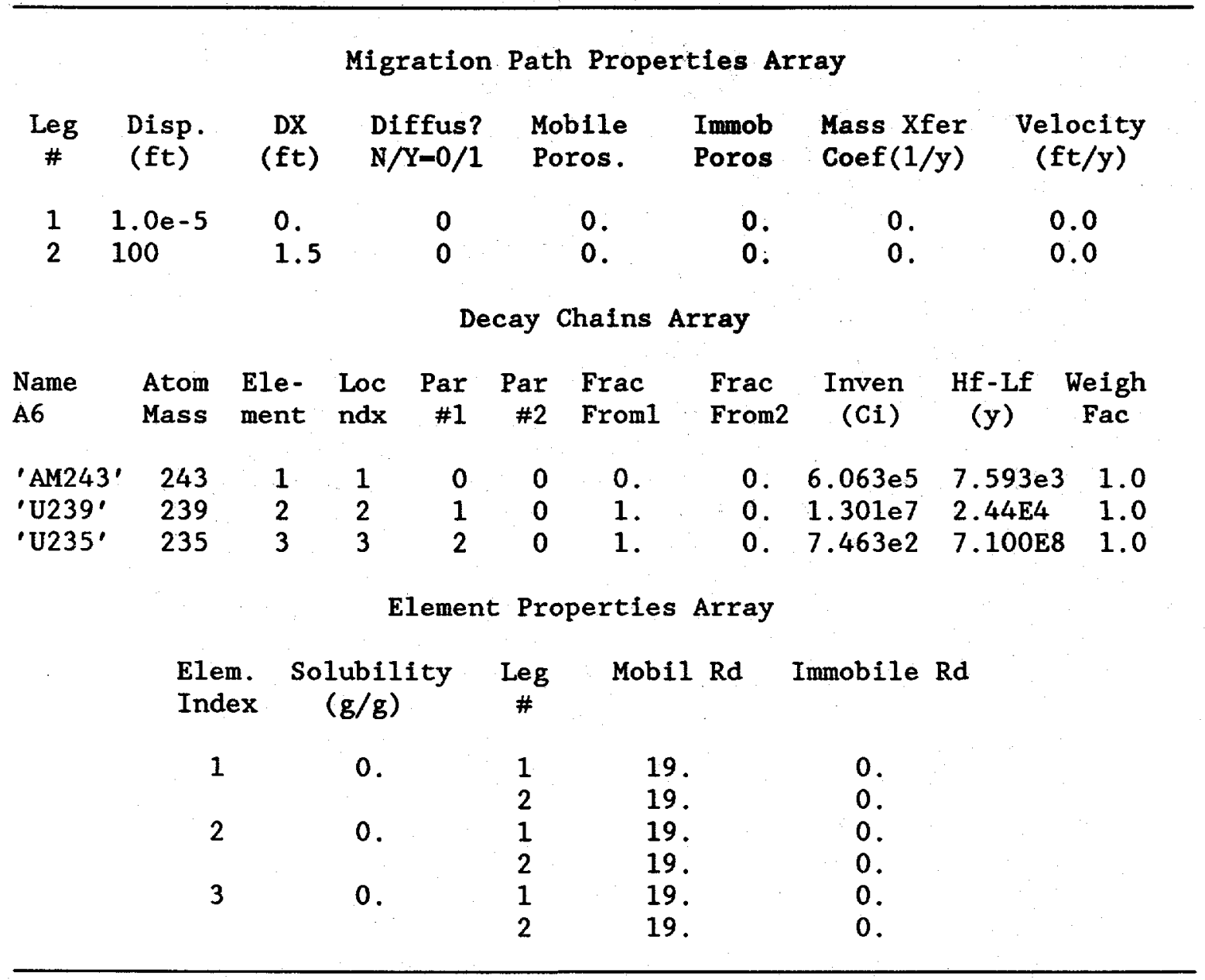

Table 5.5.

External File of Time-Varying Velocity Data

$\begin{array}{llll}50000 . & 1 & 0.098 & 1 . \\ 80000 . & 2 & 0.098 & 1 . \\ & 1 & 0.196 & 1 . \\ & 2 & 0.196 & 1 .\end{array}$


For this sample problem, the source model is assumed to be a constant leach-limited, flow-through source. This model is the best approximation to the instantaneous (in time) source boundary condition of the analyti. cal solution. However, the transport time step is also relevant for this approximation, since the source rate for each isotope is essentially "smeared" over the transport time step. Therefore, the transport time step also needs to be relatively small to try to approximate the source boundary condition. In relation to the DVM transport model, it is difficult to make the time step significantly small to approximate the instantaneous source, since numerical dispersion increases with decreasing time step (see Section 2.4.5).

Figure 5.12 shows the resultant discharge curves for ${ }^{243} \mathrm{Am},{ }^{239} \mathrm{Pu}$, and ${ }^{235 \mathrm{U}}$ from NEFTRAN II and from the analytical solution. The overall agreement between the analytical solution and NEFTRAN II is reasonable. The slight discrepancy may be due to NEFTRAN II not being able to simulate the instantaneous source term accurately. However, what is significant is that NEFTRAN II is reproducing the general shape of the analytical solution near the time the velocities are changing. This verifies that the DVM transport model for time-dependent fluid velocity conditions has implemented the corresponding mathematical model correctly. 


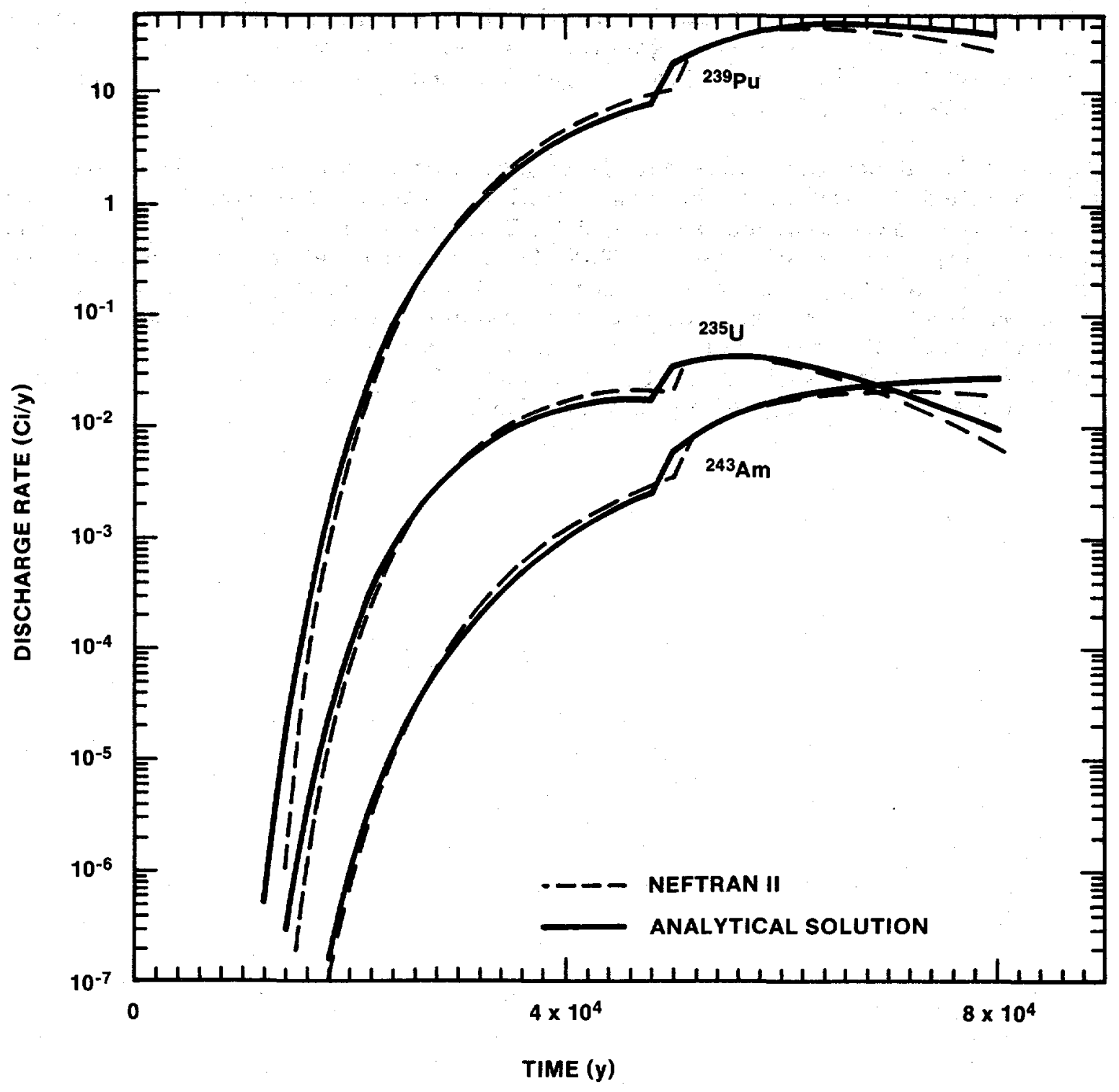

Figure 5.12. Discharge Rate Curves for Sample Problem \#3 
Abramowitz, M. and J. A. Stegun, Handbook of Mathematical Functions, Dover Publication, Inc., New York, 1965.

Bonano, E. J., P. A. Davis, L. R. Shipers, K. F. Brinster, W. E. Beyeler, C. D. Updegraff, E. R. Shepherd, L. M. Tilton, K. K. Wahi, Demonstration of a Performance Assessment Methodology for HighLevel Radioactive Waste Disposal in Basalt Formations, SAND86-2325, NUREG/CR-4759, Sandia National Laboratories, Albuquerque, NM, 1989.

Campbe11, J. E., P. C. Kaestner, B. S. Langkopf, and R. B. Lantz, Risk Methodology for Geologic Disposal of Radioactive Waste: The Network Flow and Transport (NWFT) Mode1, SAND79-1920, NUREG/CR-1190, Sandia National Laboratories, Albuquerque, NM, 1979.

Campbe11, J. E., D. E. Longsine, and M. Reeves, Risk Methodology for Geologic Disposal of Radioactive Waste: The Distributed Velocity Method of Solving the Convective-Dispersion Equation, SAND80-0717, NUREG/CR-1376, Sandia National Laboratories, Albuquerque, NM, 1980.

Campbe11, J. E. and D. E. Longsine, Distributed Velocity Method of Solving the Convective-Dispersion Equation: 2. Error Analysis and Comparison with Other Methods, Advances in Water Resources, Vol. 4, pp. $109-116,1981$.

Campbe11, J. E., D. E. Longsine, and M. Reeves, Distributed Velocity Method of Solving the Convective-Dispersion Equation: 1 . Introduction, Mathematical Theory, and Numerical Implementation, Advances in Water Resources, Vol. 4, pp. 102-108, 1981a.

Campbe11, J. E., D. E. Longsine, and R. M. Cranwe11, Risk Methodology for Geologic Disposal of Radioactive Waste: The NWFT/DVM Computer Code User's Manual, SAND81-0886, NUREG/CR-2081, Sandia National Laboratories, Albuquerque, NM, $1981 \mathrm{~b}$.

Cranwe11, R. M., J. E. Campbe11, J. C. Helton, R. L. Iman, D. E. Longsine, N. R. Ortiz, G. E. Runkle, and M. J. Shortencarrier, Risk Methodology for Geologic Disposal of Radioactive Waste: Final Report, SAND81-2573, NUREG/CR-2452, Sandia National Laboratories, Albuquerque, NM, 1982.

EPA, 40CFR191: Environmental Standards for the Management and Disposal of Spent Nuclear Fuel High-Level and Transuranic Radioactive Waste: Final Rule, Federal Register 50, No. 182, 38066-38089, 1985.

Gelbard, F., Modeling One-Dimensional Radionuclide Transport Under TimeVarying Fluid Flow Conditions, SAND89-1521, NUREG/CR-5412, Sandia National Laboratories, Albuquerque, NM, 1989.

Iman, R. L. and M. J. Shortencarrier, A FORTRAN 77 Program and User's Guide for the Generation of Latin Hypercube and Random Samples for Use With Computer Models, SAND83-2365, NUREG/CR-3624, Sandia National Laboratories, Albuquerque, NM, 1984. 
Lester, D. H., G. Jansen, and H. C. Burkholder, Migration of Radioactive Chains Through an Absorbing Medium, AICHE Symposium Series 152, Adsorption and Ion Exchange, Vo1. 71, pp. 202-213, 1975.

Longsine, D. E., E. J. Bonano, and C. P. Harlan, User's Manual for the NEFTRAN Computer Code, Sandia National Laboratories, NUREG/CR-4766, SAND86-2405, Albuquerque, NM, 1987.

Reeves, M., D. S. Ward, N. D. Johns, and R. M. Cranwe11, Theory and Implementation for SWIFT II. The Sandia Waste-Isolation Flow and Transport Mode1 for Fractured Media, Release 4.84, SAND83-1159, NUREG/CR-3328, Sandia National Laboratories, Albuquerque, NM, 1986a.

Reeves, M., D. S. Ward, N. D. Johns, and R. M. Cranwell, Data Input Guide for SWIFT II. The Sandia Waste-Isolation Flow and Transport Model for Fractured Media, Release 4.84, SAND83-0242, NUREG/CR-3162, Sandia National Laboratories, Albuquerque, NM, 1986b.

Sinnock, S, Y. T. Lin, and J. P. Brannen, Preliminary Bounds on the Expected Postclosure Performance of the Yucca Mountain Repository Site, Southern Nevada, Sandia National Laboratories, SAND84-1492, Albuquerque, NM, 1984.

Travis, B. J., S. W. Hodson, H. E. Nuttal1, T. L. Cook, and R. S. Rundberg, Preliminary Estimates of Water Flow and Radionuclide Transport in Yucca Mountain, Los Alamos National Laboratory, Los Alamos, NM, LA-UR-84-40, 1984.

Updegraff, C. D. and C. Lee, DCM3D-A Dual Continuum, 3-D Ground-Water Flow Code for Unsaturated, Fractured, Porous Media, SAND90-7015, NUREG/CR-5536, Albuquerque, NM, 1991.

van Genutchen, M. Th. and P. J. Wierenga, Mass Transfer Studies in Sorbing Porous Media, I. Analytical Solutions, Soil Science Society of America Journal, Vol. 40, No. 4, pp. 473-480, 1976. 
Application of the DVM transport model to a radioactive decay chain requires a method for determining the average velocity of atoms that decay to daughter products during a time step, $\Delta t$. Consider a chain $I_{1} \rightarrow I_{2} \rightarrow \ldots \rightarrow I_{p}$. Without loss of generality, the subscripts can be used to identify the chain (i.e., $1 \rightarrow 2 \rightarrow \ldots \rightarrow$ p). The decay rate constants are denoted by $\lambda_{1}, \lambda_{2}, \ldots, \lambda_{p}$.

For a given velocity interval in the calculation of species velocities, the averaged velocity for particles that begin $\Delta t$ as species 1 and end $\Delta t$ as species $p, v_{m}(1, \ldots, p)$, is given by

$$
v_{m}(1, \ldots, p)=\frac{1}{\Delta t} \underset{i=1}{p} \bar{T}_{i} v_{i}
$$

where $\bar{T}_{i}$ is the average time spent as species $i$ during $\Delta t, v_{i}$ the velocity of the species, and $\sum_{i=1}^{p} \bar{T}_{i}=\Delta t$.

In order to find the particle-averaged velocities, $\overline{\mathrm{T}}_{\mathfrak{i}}$ must be determined for each isotope $i$.

If species 1 survives decay to time $t_{1}$, decays to species 2 at that time, survives as species 2 to time $t_{2}$, decays to species 3 at that time, etc., then, $0=t_{0}<t_{1}<t_{2}<\ldots<t_{p}=\Delta t$ and the probability that species $i$ survives decay over its allotted time is $e^{-\lambda_{i}\left(t_{i}-t_{i-1}\right)}$.

The probability that species $i$ decays to species $i+1$ over an infinitesimal time interval, $\mathrm{dt}_{1}$, is given by $\lambda_{i} d t_{1}$.

Thus, the probability that species 1 decays to species $p$ over $\Delta t$, DP $(1, \ldots, p, \Delta t)$, is the multiple integral of the product of the following independent probabilities,

$$
\operatorname{pr}(1, \ldots, p, \Delta t)=\prod_{i=1}^{p} e^{-\lambda_{i}\left(t_{i}-t_{i-1}\right)} \underset{i=1}{p-1} \lambda_{i} \underset{i=1}{p-1} d t_{i},
$$

with integration limits $\left(0, t_{2}\right),\left(0, t_{3}\right), \ldots,\left(0, t_{p}\right)$. It can be demonstrated that by integrating the probabilities

$$
\operatorname{DP}(1, \ldots, p, \Delta t)=\int_{0}^{t_{p}} \ldots \int_{0}^{t_{2}} \operatorname{pr}(1, \ldots, p, \Delta t) .
$$


the resulting expression is given by the Bateman coefficient

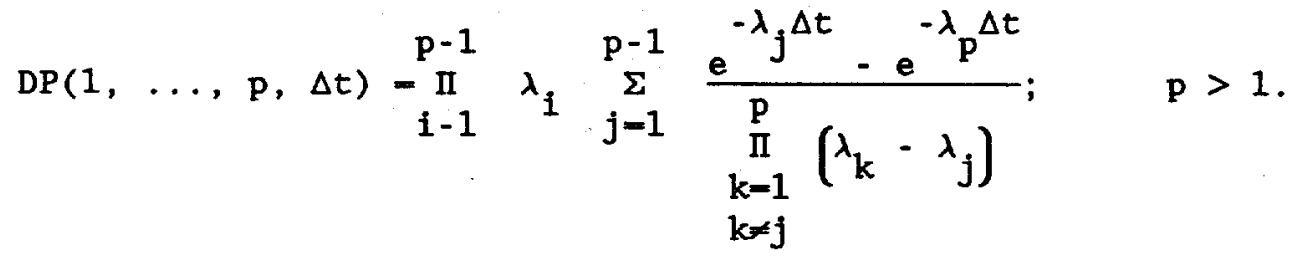

To find $\bar{T}_{1}$, the integrand $\operatorname{pr}(1, \ldots, \mathrm{p}, \Delta t)$ in Equation $A .3$ is weighted by $t_{1}-t_{1-1}$, and the resulting integral is divided by $\operatorname{DP}(1, \ldots, p, \Delta t)$. This is equivalent to the following formulation:

$$
\begin{aligned}
& \overline{\mathrm{T}}_{1}=\left[\int_{0}^{t} \mathrm{p} \int_{0}^{t_{2}} t_{1} \operatorname{pr}(1, \ldots \mathrm{p}, \Delta t)\right] / \mathrm{DP}(1, \ldots, \mathrm{p}, \Delta t), \\
& \overline{\mathrm{T}}_{\mathbf{i}}=\left[\int_{0}^{t} \mathrm{p} \ldots \int_{0}^{t_{2}} t_{i} \operatorname{pr}(1, \ldots \mathrm{p}, \Delta t)\right] / D P(1, \ldots, p, \Delta t)-\sum_{j=1}^{i-1} \overline{\mathrm{T}}_{j}
\end{aligned}
$$

for $i=2, \ldots, p-1$ and

$$
\bar{T}_{p}=\Delta t-\sum_{i=1}^{p-1} \bar{T}_{i}
$$

Before evaluating Equation A.5, one should note that for the purposes of finding the mean time spent as each isotope, the decay chain $I_{1} \rightarrow I_{2} \rightarrow \ldots \rightarrow I_{p}$ is equivalent to $1 \rightarrow 2 \rightarrow \ldots+p$ where $p=I_{p}$ and $I_{1}$, $I_{2}, \ldots, I_{p-1}$ is any permutation of the integers $1,2, \ldots, p-1$. That is, the particles must spend the same amount of time as each species from 1 to $p-1$ regardless of the order of the decay sequence. Thus, as shown below, the mean time spent as isotope 1 is found. Subsequently, the mean time spent as isotope $i$ is obtained using the same formula used to find $\mathrm{T}_{1}$ by interchanging the roles of $\lambda_{1}$ and $\lambda_{i}, i=2, \ldots, p-1$.

The general formula for the mean time spent as isotope 1 is

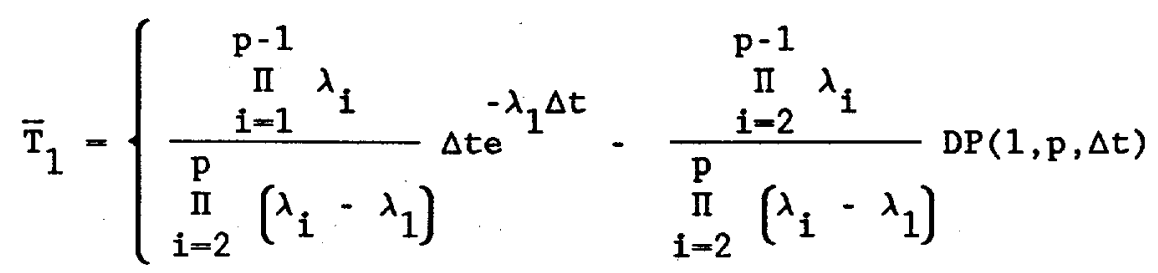




$$
\begin{aligned}
& -\frac{\prod_{i=2}^{p-2} \lambda_{i}}{\prod_{i=2}^{p-1}\left(\lambda_{i}-\lambda_{1}\right)} \mathrm{DP}(1, \mathrm{p}-1, \Delta t)-\frac{\prod_{i=2}^{\mathrm{p}-3} \lambda_{i}}{\prod_{i=2}^{\mathrm{p}-2}\left(\lambda_{i}-\lambda_{1}\right)} \cdot \mathrm{DP}(1, \mathrm{p}-2, \mathrm{p}-1, \mathrm{p}, \Delta t) \\
& \left.\ldots \frac{1}{\lambda_{2}-\lambda_{1}} \operatorname{DP}(1, \ldots, p, \Delta t)\right\} / \mathrm{DP}(1, \ldots, \mathrm{p}, \Delta t)
\end{aligned}
$$

where $\operatorname{DP}(1, p, \Delta t)$ denotes the production factor that would result from decay directly from species 1 to species $p$. That is, assume species 2, $\ldots, p-1$ are not present in the decay chain so that $I_{1} \rightarrow I_{p}$. The factor DP $(1, p, \Delta t)$ is then defined by

$$
\operatorname{DP}(1, \mathrm{p}, \Delta t)=\lambda_{1} \frac{e^{-\lambda_{1} \Delta t}-e^{-\lambda} p^{\Delta t}}{\lambda_{p}-\lambda_{1}} .
$$

Similar definitions are used for DP $(1, p-1, p, \Delta t), D P(1, p-2, p-1, p$, $\Delta t)$, etc.

In order to reduce numerical errors as much as possible, the exponential terms in Equation A.7, are evaluated using an infinite series. Terms in the infinite series with identical powers of $\Delta t$ are collected to create a single series which is then evaluated. The complexity of the resulting series is dependent on the value of $p$. Currently, NEFTRAN II only implements this approach for $\mathrm{p} \leq 5$.

Encountering the need to transport a six-member subchain is not anticipated and, in fact, has never been noted. In NEFTRAN II, however, an approximate scheme for subchains of length 6 or greater has been implemented. This scheme is also used if it is judged possible that convergence of the above-mentioned series will be too slow or numerically infeasible. Comparing mean-lives of the species to equal shares of the time step, one of three possibilities must be true:
(a) $\frac{1}{\lambda_{i}}>\frac{\Delta t}{p}$
for $a 11 i=1, \ldots, p$
(b) $\frac{1}{\lambda_{i}} \leq \frac{\Delta t}{p}$
for all $1=1, \ldots, p$
(c) for some isotopes $\mathrm{k}$,
$\frac{1}{\lambda_{k}} \leq \frac{\Delta t}{p}$ and for the remainder $j, \frac{1}{\lambda_{j}}>\frac{\Delta t}{p}$ 
In case (a) it can be shown that setting

$$
\overline{\mathrm{T}}_{1}-\frac{\Delta t}{\mathrm{p}}
$$

for all $1=1, \ldots, p$ is a good approximation. One should note that in this case, average velocities are simply arithmetic averages. In case (b) a good approximation is to set

$$
\overline{\mathrm{T}}_{i}=\frac{1 / \lambda_{i}}{\overline{\mathrm{T}}} \Delta t \text {, }
$$

where

$$
\bar{T}=\sum_{i=1}^{p} 1 / \lambda_{i}
$$

That is, the time spent as species $i$ during $\Delta t$ is set to a fraction of $\Delta t$ defined by the ratio of the mean-life of species $i$ to the sum of all mean-lives. Finally, for case (c) those species having the smaller meanlives are assigned their mean-life,

$$
\overline{\mathrm{T}}_{\mathrm{k}}=\frac{1}{\lambda_{\mathrm{k}}}
$$

and the time remaining in $\Delta t$,

$$
\mathrm{TR}=\Delta t-\sum_{\mathrm{k}} \frac{1}{\lambda_{\mathrm{k}}}
$$

is divided evenly among the species with larger mean-lives

$$
\overline{\mathbf{T}}_{\mathbf{j}}=\frac{\mathrm{TR}}{\mathrm{p}-\mathrm{m}}
$$

where $\mathrm{m}$ is the number of species having the smaller mean-lives. 
The discharge model contained in NEFTRAN II consists of multiplying the number of atoms of species $r$, at time $t$, in grid block $N_{x}$ by the fraction of species $r$ - $p$ in grid block $N_{x}$ that discharge as species $r$. These set of fractions are stored in array $F$. The determination of array $F$ is the subject of this appendix.

$F$ can be determined in a potentially different manner for each different number of precursors (p). However, with simplifying assumptions concerning velocities and production, it is possible to reduce the cases to $\mathrm{p}=0$ and $\mathrm{p} \geq 1$.

Case $1(p=0)$. Here, only atoms of species $r$ at $t$, that discharge as species $r$ during the time increment $\Delta t$ are of concern. Recall that each grid block has its contents of species $r$ partitioned into $\mathrm{N}_{\mathrm{v}}$ equally weighted velocity packets. Packet $\mathbf{j}$ is assigned velocity $v(j, r), j=1$, $\ldots, N_{v}$. So packet $j$ in grid block $N_{x}-1+1$ can contribute to the discharge of species $r$ if

$$
v(j, r) \cdot \Delta t \geq(i-1) \cdot \Delta x
$$

The measure of its contribution and the time required to discharge depends on the Courant number (CN),

$$
C N(j, r)=v(j, r) \bullet \frac{\Delta t}{\Delta x} \bullet \geq(i-1) \text { for contribution }
$$

A packet may totally or partially traverse the boundary as shown in Figure B.1. The contribution factor of packet $j$ in grid block $N_{x}-i+1$ is then,

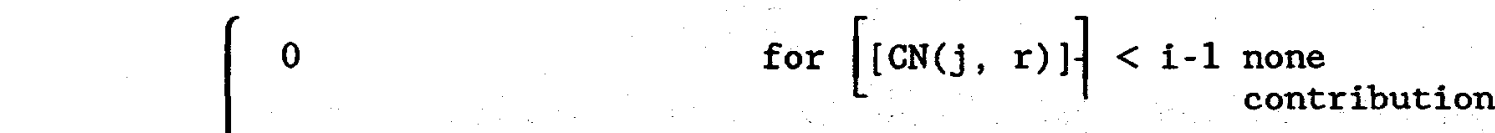

$$
\begin{aligned}
& h(j, r)=\{\mathrm{CN}(j, r)-[[\mathrm{CN}(j, r)]] \text { for }[[\mathrm{CN}(j, r)]]=i-1 \text { partial } \\
& 1 \text { for }[[C N(j, r)]]=i=1 \text { total }
\end{aligned}
$$

where $[[\mathrm{CN}]]$ is the greatest integer less than or equal to $\mathrm{CN}$. 

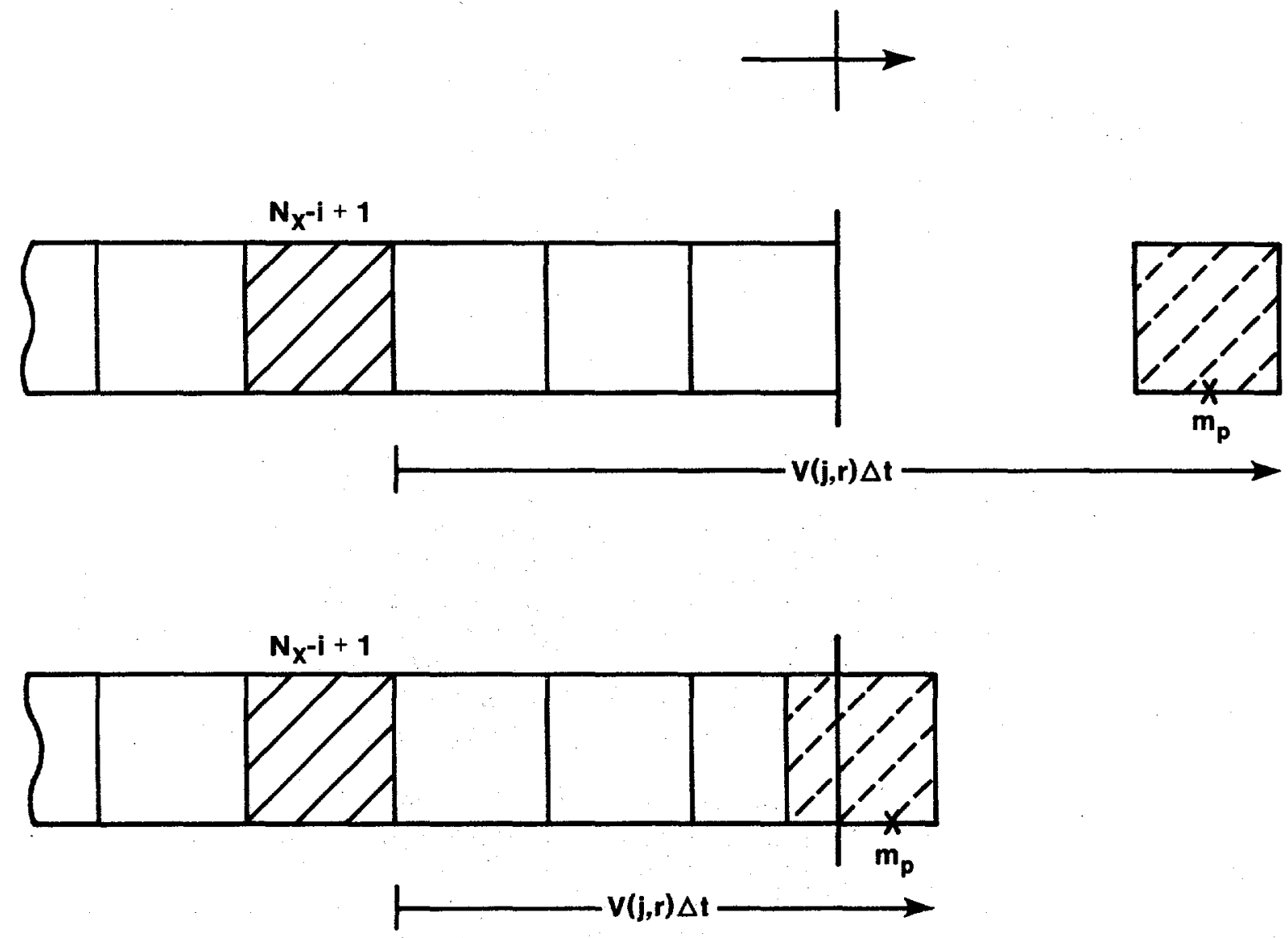

Figure B.1. Total and Partial Discharge of a Typical Velocity Packet 
The time to discharge, $t(j, r, i)$, is defined as the time at which the spatial midpoint, $m_{p}$, crosses the boundary. That is,

$$
t(j, r, i)= \begin{cases}\frac{i-\frac{1}{2}}{C N(j, r)} \Delta t & \text { for }[[C N(j, r)]]>i-1 \\ \frac{C N(j, r)+i-1}{2 C N(j, r)} \Delta t & \text { for }[[C N(j, r)]]=i-1\end{cases}
$$

This time is useful because of the decay of species $r$. Recalling that packet $j$ is weighted by $\left(\mathrm{N}_{\mathrm{v}}\right)^{-1}$, we write

$$
F(r, r, i)=\frac{1}{N_{v}} \sum_{j=1}^{N_{v}} h(j, r) e^{-\lambda} t(j, r, i)
$$

Note that $\mathrm{NB}(r, r)=\left[\left[\mathrm{CN}\left(\mathrm{N}_{\mathrm{v}}, \mathrm{r}\right)+1\right]\right]$.

Case $2 p \geq 1$. Consider the subcase $p=1$ as a model. Suppose an atom of species $r-1$ at the beginning of time interval $\Delta t$ is distance $L$ from the discharge point. We determine the probability that this atom decays to species $r$ and discharges $1+i d$ as the product of probabilities of the following three independent events.

Event 1. The atom is of specles type $r-1$ after time $t_{d}$,

$$
0<t_{d}<\Delta t
$$

Event 2. During infinitesimal time $d t_{d}$, the atom decays to species type $r$.

Event 3. The atom remains as species $r$ in the time remaining to discharge.

These events have the probabilities:

$$
\begin{aligned}
& \operatorname{pr}\left(\text { Event 1) }=e^{-\lambda} r-1 t_{d}\right. \\
& \operatorname{pr}\left(\text { Event 2) }=f(r, r-1) \lambda_{r}-1 d t_{d}\right. \\
& \operatorname{pr}\left(\text { Event 3) }=e^{-\lambda_{r} t_{R}}\right.
\end{aligned}
$$


where $f(r, r-1)$ is the decay fraction of species $r-1$ to $r$ and $t_{R}$ is the time remaining to discharge, i.e.,

$$
t_{R}=\frac{L-v(r-1) t_{d}}{v(r)}
$$

Note that $v(r)$ and $v(r-1)$ are picked from the velocity distributions for species $r$ and $r-1$. However, they are chosen from the same velocity subinterval and thus the velocity index $j$ is dropped.

The production fraction of species $r$ by species $r-1$ is then found by integrating over possible time (i.e., time range during which atoms of species $r$ - 1 can remain as such and still discharge as species $r$ ),

$$
\begin{aligned}
& P(r, r-1)=\lambda_{r}-1 \text { f(r, r - 1) } \int_{T_{1}}^{T} e^{-\lambda r-1 t_{d}-\lambda r \frac{\left(L-v(r-1) t_{d}\right)}{v(r)}} d t d \\
& -\frac{\lambda_{r} L}{v(r)} \\
& =\lambda_{r}-1^{f(r, r-1) e} \\
& \text { - } \quad\left(\frac{1 / v(r-1)}{\frac{\lambda_{r}}{v(r)}-\frac{\lambda_{r}-1}{v(r-1)}}\right) e^{\left(\frac{\lambda_{r}}{v(r)}-\frac{\lambda_{r}-1}{v(r-1)}\right.} v(r-1) t_{d} \mid T_{2}
\end{aligned}
$$

The above integration is valid as long as

$$
\frac{\lambda_{r}}{v(r)} \neq \frac{\lambda_{r}-1}{v(r-1)}
$$

Otherwise, Equation B. 9 is replaced by

$$
P(r, r-1)=\lambda_{r}-1 f(r, r-1) e^{-\frac{\lambda_{r} L}{v(r)}}\left(T_{2}-T_{1}\right)
$$

Here, $T_{1}$ and $T_{2}$ define the time range during which atoms of species $r-1$ can remain as such and still discharge as species $r$.

There are two conditions that must be met to define $T_{1}$ and $T_{2}$. First, one of the velocities must be large enough so that atoms traveling with 
that velocity can reach the discharge boundary. Second, the velocity of the daughter isotope, $r$, must be positive. Relative to the latter, recall that velocities are normally distributed. There are combinations of dispersivity, mean velocity, and time step that will produce negative distributed velocities.

Mathematically, the first criterion above means that $\max \left(v_{r-1}, v_{r}\right) \bullet \Delta t \geq L$ and the second means $v_{x} \geq 0$. With these two criteria met, the following table defines the values $\mathrm{T}_{1}$ and $\mathrm{T}_{2}$ :

$$
v_{r-1}>v_{r} \quad v_{r}>v_{r-1}>0 \quad v_{r}>0>v_{r-1}
$$

\begin{tabular}{|c|c|c|}
\hline $\max (0, t)$ & 0 & 0 \\
\hline$\stackrel{\mathrm{L} / \mathrm{v}}{\mathbf{r}-1}$ & $\min (L / v) r-1{ }^{t}$ & t \\
\hline
\end{tabular}

where $t_{I}=\left(L-v_{r} \Delta t\right) /\left(v_{r-1}-v_{r}\right)$.

The basis for $T_{1}$ and $T_{2}$ is shown for one case $\left(v_{r-1}>v_{r}\right)$. The other cases follow from similar reasoning. For the first condition above, $v_{r-1} \Delta t \geq L$. Thus, the atoms can reach the boundary traveling as the parent ( $r-1)$; however, they must not cross the boundary prior to decaying to $r$. The latest time that decay can occur is therefore given by the travel time to the boundary, $\mathrm{T}_{2}=\mathrm{L} / \mathrm{v}_{\mathrm{r}-1}$. On the other hand, it is not known from the conditions stated whether atoms can reach the boundary traveling as the daughter. If they can, then decay can occur instantaneously so that $\mathrm{T}_{1}=0$. If not, then the atoms must travel for sufficient distance as the parent so that when decay does occur, the distance remaining to the discharge boundary can be covered traveling as the daughter in the time remaining in the time step. This condition can be stated as:

$$
v_{r-1} T_{1}+v_{r}\left(\Delta t-T_{1}\right)=L
$$

Solving for $T_{1}$ gives the expression for $t_{I}$ above. Note that the condition on the adequacy of the daughter velocity to displace atoms to the discharge point is accounted for by taking the maximum value as shown in the first column.

In order to generalize Equations B.9 and B.11 to cases p $>1$, one could consider the set

$$
\{t(r-p), \ldots, t(r-1)\}
$$


where $t(r-k)$ denotes the time interval over which species $r-k$ does not decay and travels with velocity $v(r-k), k=i, \ldots, p$. Then $d t(r-$ $k$ ) would be that infinitesimal time for decay from species $r$ - $k$ to species $r-(k-1)$. In this fashion $p-1$ nested integrals must be evaluated. However, finding the $2(p-1)$ limits of integration then becomes a formidable task.

As an option we treat the decay of species

$$
r-p \rightarrow r-(p-1) \rightarrow \ldots \rightarrow r-1
$$

as a single process over time

$$
t_{d}=\sum_{k=1}^{p} t(r-k)
$$

The velocity during time $t_{d}$ is approximated by the arithmetic average of species' velocities, i.e., define $v(r p)$ by

$$
v(r p)=\frac{1}{p} \sum_{k=1}^{p} v(r-k)
$$

This is a reasonable approximation for small $t_{d}$ (see Appendix A, Species Velocity Mode1), which is generally the case in this analysis.

Event 1 is then restated as:

Event $1^{\prime}:$ The atom begins $\Delta t$ as type $r-p$ and is of type $r-1$ at $t_{d}$.

Events 2 and 3 remain the same with $t_{R}$ replaced by

$$
t_{R}=\frac{L-v(r p) t}{v(r)} d
$$

The probability of event $1^{\prime}$ is the Bateman production coefficient over $t_{d}$,

$$
\underset{k=2}{\mathrm{p}} f(r-k, r-(k-1)) \lambda_{r-k} \sum_{\ell=2}^{p}\left\{\frac{e^{-\lambda} r-\ell^{t}-e^{-\lambda} r-1}{\prod_{m=1}^{p}\left(\lambda_{r-m}-\lambda_{r-l}\right)}\right\}
$$


where $f(r-k, r-(k-1))$ is the decay fraction of species $r-k$ to species $r-(k-1)$. The production fraction is then

$$
\begin{aligned}
& P(r, r-p)=\prod_{k=1}^{p} f(r-k, r-(k-1)) \lambda_{r-k}
\end{aligned}
$$

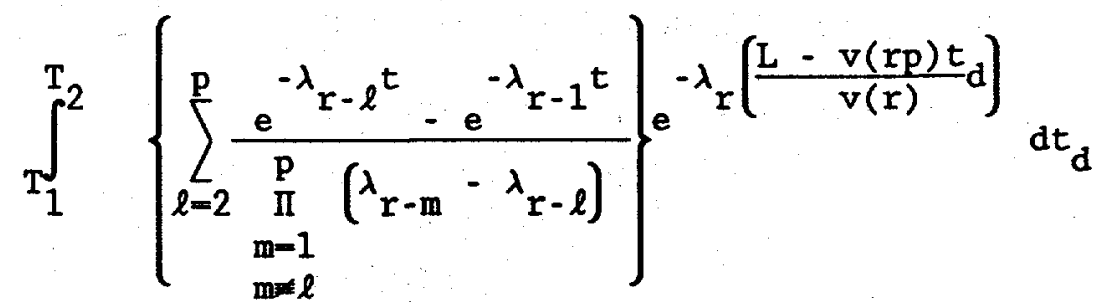

If $\frac{\lambda_{r}}{v(r)} \neq \frac{\lambda_{r}-j}{v(r p)}$ for all $k-1, \ldots, p$, then integrating Equation B.17 and rearranging terms yields

$$
\begin{aligned}
& P(r, r-p)=\prod_{k=1}^{p} f(r-k, r-(k-1)) \lambda_{r-k} e^{-\frac{\lambda_{r}}{v(r)} L}
\end{aligned}
$$

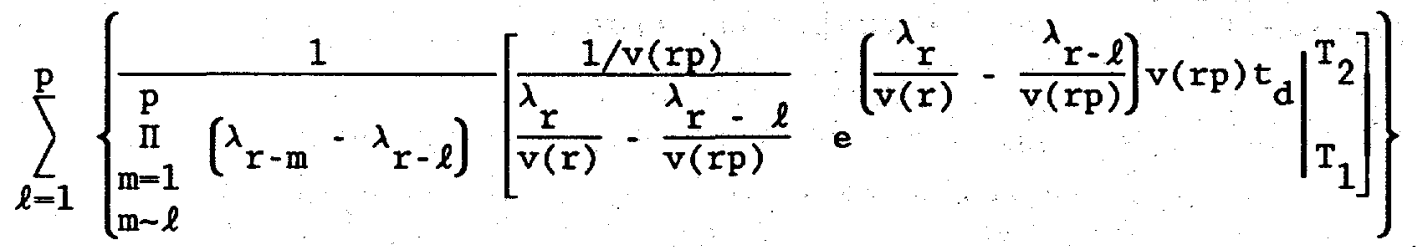

If for $p=1$ we define $\underset{\substack{m=1 \\ m-l}}{\stackrel{p}{I}}\left(\lambda_{r-m}-\lambda_{r-l}\right)=1$, then Equation B.18 contains Equation B.9.

If for some $\ell=\ell^{\prime}, \lambda_{r} / v(r)-\lambda_{r-\ell^{\prime}} / v(r p)^{\prime}$ then the sum $\sum_{\ell=1}^{p}$ is replaced by p

$\Sigma$ and the term

$\ell=1$

$\ell \neq \ell^{\prime}$ 


$$
\prod_{k=1}^{p} f(r-k, r-(k-1)) \lambda_{r-k} e^{-\frac{\lambda_{r}}{v(r)} L} \frac{T_{2}-T_{1}}{\prod_{\substack{m=1 \\ m \neq \ell^{\prime}}}^{p}\left(\lambda_{r-m}-\lambda_{r-\ell^{\prime}}\right)}
$$

is added.

The determination of $T_{1}$ and $T_{2}$ proceeds as above with $v(r-1)$ replaced by $\mathrm{v}(\mathrm{rp})$.

Since $v(r)=v(j, r)$ for some velocity interval $j=1, \ldots, N, v(r p)$ and thus $P(r, r-p)$ depend on $j$. For a given grid block index $i$ define the distance

$$
L=\left(i-\frac{1}{2}\right) \Delta x
$$

and factor

$P^{\prime}(j, r, r-p)= \begin{cases}P(j, r, r-p) & (\text { evaluated at } L) \text { if } P(j, r, r-p)>0 \\ 0 & , \text { otherwise . }\end{cases}$

Finally, array $F$ is defined by

$$
F(r, r-p, i)=\frac{1}{N_{v}} \sum_{j=1}^{N_{v}} P^{\prime}(j, r, r-p)
$$

It may happen that $v(j, r)$ and $v(j, r p)$ are such that

$$
0<v(j, r) \Delta t, v(j, r p) \Delta t<\Delta x / 2
$$

for all $j=1, \ldots, N_{v}$. In this case, some of species $r$ in grid block $N_{x}$ should discharge during $\Delta t$. However, because we take $L$ as the distance from the grid block midpoint to the discharge location (i.e., for block $\left.\mathrm{N}_{\mathrm{x}}, \mathrm{L}=\Delta \mathrm{x} / 2\right)$, the discharge model presented above would produce no discharge of species $r$. In this special case, $L$ is decreased to $L^{\prime}$ such that

$$
v(j, r) \Delta t>L^{\prime} \quad \text { or } \quad v(j, r p) \Delta t>L^{\prime}
$$

for some $j$ values. Then for grid block $N_{x}(i . e ., i=1)$, 


$$
\left.F(r, r-p, i)=\frac{L^{\prime}}{L} \frac{1}{N_{v}} \sum_{j=1}^{N_{v}} P^{\prime}(j, r, r-p) \text { (evaluated at } L^{\prime}\right)
$$

Further, note that $\mathrm{NB}(r, r-p)$ can be defined by the maximum value of $i$ that yields $P^{\prime}(j, r, r-p) \neq 0$ for any $j$.

Recalling that Equation B.18 contains Equation B.9, Equations B.18 and B.23 completely define array $F$. 


\section{APPENDIX C \\ Distribution of Source Into Migration \\ Path Using Mixing-Cell Model}

The mixing-cell source model provides constant, average source rates for radionuclides during each time step, $\Delta t$. As radionuclides exit the cell, they enter a ground-water travel path to the accessible environment. Radionuclides traveling along this path are subject to convection, dispersion, retardation, and decay. If an atom exits the mixing cell at time $t_{c}, t \leq t_{c} \leq t+\Delta t$, its location at $t+\Delta t$ is subject to all of these processes. The model described below keeps track of the movement of source particles and thus is able to spatially distribute the mass of a species over each $\Delta t$.

A source factor array $S_{F}$ is generated during the initial execution phase of NEFTRAN II. It theoretically has three indices,

$$
s_{F}=s_{F}(i, \ell, k)
$$

where the mass of species $i$ in grid block $k$ will be incremented by fraction $S_{F}(i, l, k)$ applied to the average source of the parent species $l$. Letting $\rho(i, k, t)$ be the atoms of species $i$ in grid block $k$ at time $t$, this at time $t+\Delta t$ is given by

$$
\rho(i, k, t+\Delta t)=\rho(i, k, t)+\sum_{j=0}^{P_{i}} S_{F}(i, i-j, k) \bar{S}(i-j)
$$

where $P_{i}$ is the number of parents necessary to describe production of species $i$ over $\Delta t$ and $S(i-j)$ is the average source of parent $i-j$ over $\Delta t$. $S_{F}$ is determined separately for the three cases $j=0, j=1, j>1$.

The DVM technique for radionuclide transport treats convection and retardation by assigning mean velocities to each species, and dispersion is modeled by distribution species velocities about the mean velocity. The species velocities are assumed to have equal probability and there are in general $\mathrm{N}_{\mathrm{v}}$ such values (see Section 2.4).

$v_{b}^{i}$ denotes the velocity of species $i$ in velocity bin $b, b=1, \ldots, N_{v}$. Each atom of species $i$ is therefore assigned a velocity $v_{1}$, for a given $b$ after the atom exits the mixing cell at time $t_{c}$.

First, the case $j=0$ will be examined. The fraction $S_{F}(i, i, k)$ for grid block $k$ is found by integrating the product of the following three probability functions over the total time necessary for particles to be in grid block $k$ at the end of $\Delta t$. 
1. The probability that an atom exits the ce11 during the infinitesimal time $d t_{c}$ about $t_{c}$ is

$$
\mathrm{dt}_{\mathrm{c}} / \Delta \mathrm{t}
$$

since $\overline{\mathrm{S}}$ is constant over $\Delta t$.

2. The probability that an atom of species 1 survives decay from $t_{c}$ to the end of $\Delta t$ is

$$
e^{-\lambda_{i}\left(\Delta t-t_{c}\right)}
$$

3. The probability that an atom of species $i$ is assigned to velocity bin $b$ is $1 / N_{v}$.

Therefore, the probability that an atom of species $i$ in velocity bin $b$ exits the cell during $\Delta t$ and occupies grid block $k$ at species $i$ at the end of $\Delta t$ is

$$
\operatorname{pr}(i, b, k)=\frac{1}{N_{v} \Delta t} \int_{T_{1}}^{T_{2}} e^{-\lambda_{i}\left(W t-t_{c}\right) d t} c
$$

where $T_{1}$ and $T_{2}$ define the time interval over which an atom can cross the cell boundary and end up in grid block $k$ during $\Delta t$.

Integration of Equation $C .5$ from $T_{1}$ to $T_{2}$ yields

$$
\operatorname{pr}(\mathrm{i}, \mathrm{b}, \mathrm{k})=\frac{1}{\lambda_{i} \Delta t \mathrm{~N}_{\mathrm{v}}}\left(\mathrm{e}^{-\lambda_{i}\left(\Delta t-\mathrm{T}_{2}\right)}-\mathrm{e}^{-\lambda_{i}\left(\Delta t-\mathrm{T}_{1}\right)}\right)
$$

It remains to find $T_{1}$ and $T_{2}$. First, one should note the grid block numbering system of Figure C.1. Following the usual treatment of dispersion governed by Fick's first law of diffusion, it is sometimes necessary to allow particles to be dispersed upstream in the migration path. Therefore, some material may appear to move in a negative direction, which is solely the effect of dispersion. This does not imply that particles reenter the cell. Nevertheless, grid block $k$ represents the spatial increment from $k \Delta x$ to $(k+1) \Delta x$. Further, only particles assigned positive velocities can end up in positive blocks, and those with negative velocities in negative blocks. 
MIXING CELL
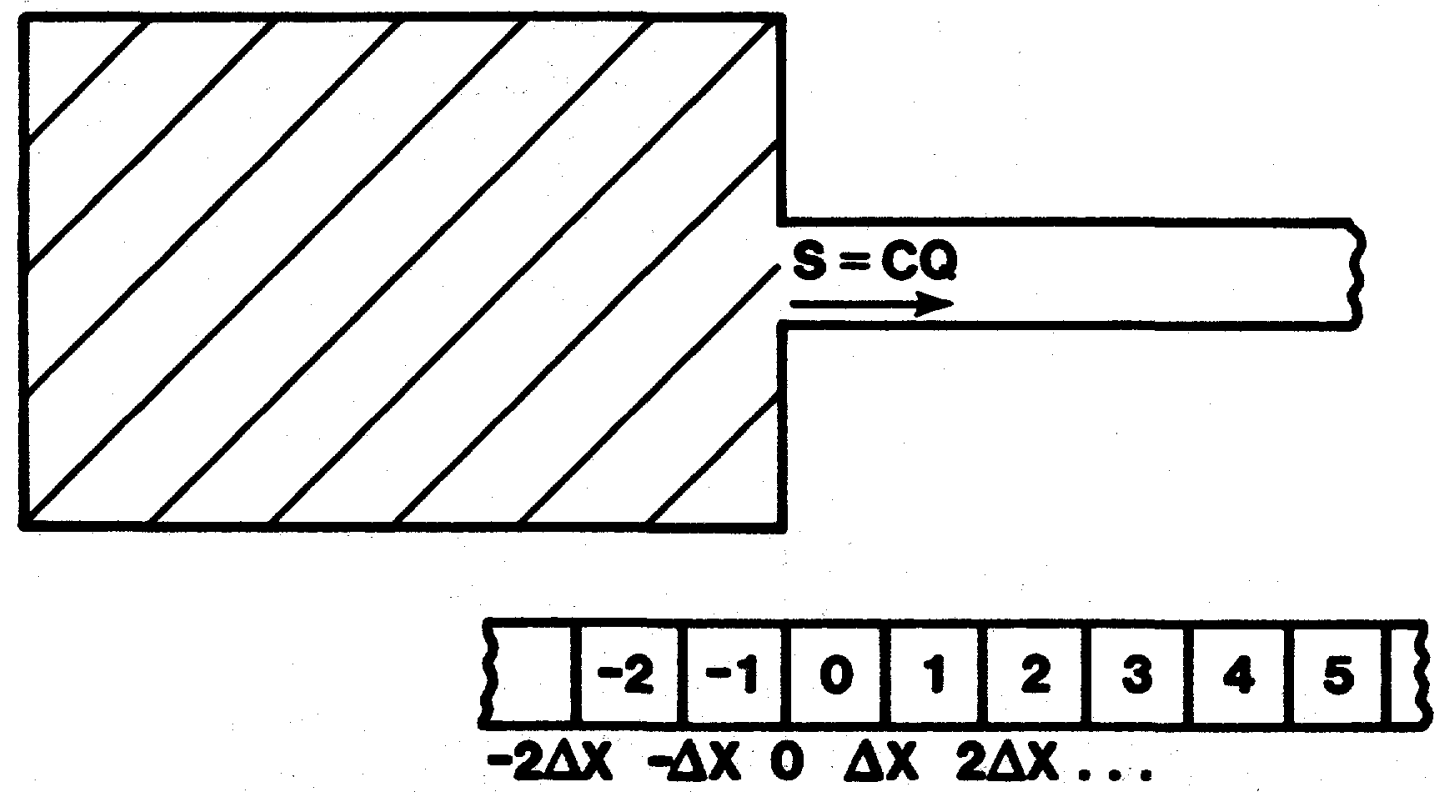

Figure C.1. The Grid Block Numbering Convention

Times $T_{1}$ and $T_{2}$ are found by noting that the distance traveled by an atom of species $i$ in velocity bin $b$ must be bounded by $k \Delta x$ and $(k+1) \Delta x$ to reside in block $k$ at the end of $\Delta t$. That is,

$$
k \Delta x \leq v_{i}^{b}\left(\Delta t-t_{c}\right) \leq(k+1) \Delta x
$$

or

$$
k \Delta x-v_{i}^{b} \Delta t \leq-v_{i}^{b} t c \leq(k+1) \Delta x-v_{i}^{b} \Delta t
$$

If $\mathbf{v}_{i}^{b}>0$,

$$
\Delta t-\frac{(k+1) \Delta x}{v_{i}^{b}} \leq t_{c} \leq \Delta t-\frac{k \Delta x}{v_{i}^{b}}
$$

and if $v_{b}^{i}<0$ 


$$
\Delta t-\frac{\mathrm{k} \Delta \mathrm{x}}{\mathrm{v}_{\mathrm{b}}^{\mathbf{i}}} \leq \mathrm{t}_{\mathrm{c}} \leq \Delta t-\frac{(\mathrm{k}+1) \Delta \mathrm{x}}{\mathrm{v}_{\mathrm{b}}^{\mathbf{i}}} .
$$

The bounds on $t_{c}$ given by Equations $c .8$ and $C .9$ are $\Delta t$ less the travel times from the cell to the edges of grid block $k$. These bounds are labeled

$$
t_{1}=\Delta t-\frac{(k+1) \Delta t}{v_{i}^{b}} \text { and } t_{2}=\Delta t-\frac{k \Delta x}{v_{i}^{b}}
$$

respectively. From the grid block numbering convention $t_{1} \leq \Delta t$ and $t_{2} \leq \Delta t$. The conditions $v_{1}^{b}>0$ and $v_{1}^{b}<0$ can be combined by defining $T_{1}$ and $\mathrm{T}_{2}$ as follows

$$
\begin{aligned}
& T_{1}=\max \left\{\min \left(t_{1}, t_{2}\right), 0\right\} \\
& T_{2}=\max \left(t_{1}, t_{2}\right\} .
\end{aligned}
$$

If $t_{2} \leq 0$ there is no contribution from velocity bin $b$ to grid block $k$. In that case, set

$$
\operatorname{pr}(i, b, k)=0 \quad \text {. }
$$

Finally define

$$
S_{F}(i, i, k)=\sum_{b=1}^{N_{v}} \operatorname{pr}(i, b, k)
$$

for each block $k$. Since $j=0$, that is species $i$ has no parents, it follows from Equation $C .2$ that the second index for $S_{F}$ must be $i$.

Next, the case $j=1$ (i.e., a two-member chain) is examined. For convenience, in the notation species $i$ is assumed to decay to species 2 . The change in mass of species 2 in each grid block $k$ due to the radioactive decay of species $i$ must be determined. In finding $S$, it is assumed that decay and production can only occur after an atom exits the ce11. Therefore, if $t_{d}$ is the time at which decay occurs, then $t_{d} \geq t_{c}$.

The probability that an atom leaving the cell as species 1 resides in block $k$ as species 2 at the end of $\Delta t$ is the integral of the product of the following five probabilities. 
1. The probability that an atom of species 1 exits the cell during $d t_{c}$ about $t_{c}$ is

$$
d t_{c} / \Delta t
$$

2. The probability that an atom of species 1 survives decay from $t_{c}$ to $t_{d}$ is

$$
e^{-\lambda_{1}\left(t_{d}-t_{c}\right)}
$$

3. The probability that an atom of species 1 decays to species 2 during $d t_{d}$ about $t_{d}$ is

$$
h_{1,2} \lambda_{1} d t_{d}
$$

where $h_{1,2}$ is the branching fraction from species 1 to species 2 .

4. The probability that an atom of species 2 survives decay from $t_{d}$ to $\Delta t$ is

$$
e^{-\lambda_{2}\left(\Delta t-t_{d}\right)}
$$

5. The probability that an atom is assigned to velocity bin $b$ is $1 / \mathrm{N}_{\mathrm{v}}$. It is assumed that, if an atom is assigned velocity $v_{1}^{b}$, then it doesn't change bins after decay. That is, as species 2, it travels with velocity vo.

Therefore, the probability that a source particle of species 1 ends up in block $k$ as species 2 is

$$
\begin{aligned}
\operatorname{pr}(2,1, k, b) & =\frac{h_{1,2} \lambda_{1} e^{-\lambda} N_{2} t}{\Delta t N_{v}} \int_{T_{d_{1}}}^{T_{d_{2}}} \int_{T_{c_{1}}}^{T_{c_{2}}} e^{\lambda_{1} t_{c}} \\
& \cdot e^{-\left(\lambda_{1}-\lambda_{2}\right) \cdot t_{d} d t_{c} d t} d,
\end{aligned}
$$

where $T_{c_{1}}, T_{c_{2}}, T_{d_{1}}, T_{d_{2}}$, define the time ranges to exit the cell and decay so ${ }^{1}$ that a particle may occupy block $k$ at the end of $\Delta t$. 
In order to solve Equation C.14, the limits of integration must be determined. Disregarding the dependence on $K$, the integration limits would be

$$
\begin{array}{ll}
T_{c_{1}}=0 & T_{d_{1}}=0 \\
T_{c_{2}}=t_{d} & T_{d_{2}}=\Delta t
\end{array}
$$

Applying these limits to Equation C.14 then gives the total amount of species 2 produced from decay of species 1 . The integration limits are graphically illustrated in Figure C. 2 .

As well be shown, for an atom to end up in grid block $k$, the area of integration must also be bounded by a pair of parallel lines. THe manner in which these lines intersect the shaded triangle in Figure C.2 results in 36 different sets of limits for $\mathrm{T}_{\mathrm{d}_{1}}, \mathrm{~T}_{\mathrm{d}_{2}}, \mathrm{~T}_{\mathrm{c}_{1}}$, and $\mathrm{T}_{\mathrm{c}_{2}}$.

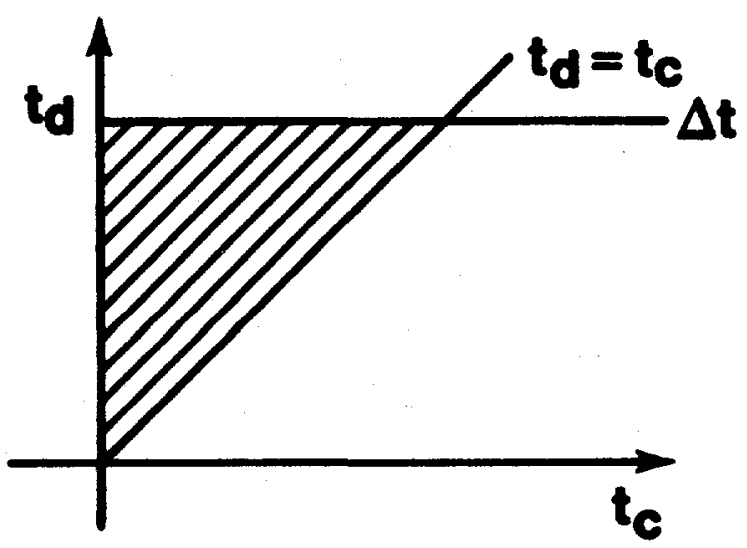

Figure C.2. Shaded Area Represents the Region of Integration for Total Source of Species 2 from Species 1 
First, the parallel lines must be determined. The total distance traveled by an atom in velocity bin $b$ is

$$
v_{1}^{b}\left(t_{d}-t_{c}\right)+v_{2}^{b}\left(\Delta t-t_{d}\right)
$$

The superscript $b$ will be dropped for convenience. In order to occupy block $k$, the following condition must be satisfied

$$
k \Delta x \leq v_{1}\left(t_{d}-t_{c}\right)+v_{2}\left(\Delta t-t_{d}\right) \leq(k+1) \Delta x
$$

Solving for $t_{d}$ directly is possible only when $v_{1} \neq v_{2}$. If $v_{1}>v_{2}$, Equation C. 15 becomes

$\frac{k \Delta x-v_{2} \Delta t}{v_{1}-v_{2}}+\left(\frac{v_{1}}{v_{1}-v_{2}}\right) t_{c} \leq t_{d} \leq\left(\frac{v_{1}}{v_{1}-v_{2}}\right) t_{c}+\frac{(k+1) \Delta x-v_{2} \Delta t}{v_{1}-v_{2}}$.

If $v_{1}<v_{2}$, Equation C.15 becomes

$\frac{(k+1) \Delta x-v_{2} \Delta t}{v_{1}-v_{2}}+\left(\frac{v_{1}}{v_{1}-v_{2}}\right) t_{c} \leq t_{d} \leq\left(\frac{v_{1}}{v_{1}-v_{2}}\right) t_{c}+\frac{k \Delta x-v_{2} \Delta t}{v_{1}-v_{2}}$

If $v_{1}=v_{2}$, Equation $C .15$ becomes

$$
T_{1} \leq t_{c} \leq T_{2}
$$

where $T_{1}$ and $T_{2}$ are defined by Equations C.10 and $C .11$, respectively, when $v_{1}^{b}=v_{1}=v_{2}$. Equation C.18 implies 1imits of integration on $t_{d}$ because $t_{d} \geq t_{c}$. Equations $C .16$ and $C .17$ are lines with the same slope $\frac{v_{1}}{v_{1}-v_{2}}$

Condition $1\left(v_{1}=v_{2}\right)$ corresponding to Equation C.18 is examined first. If $\mathrm{T}_{2} \leq 0$, there is no contribution to grid block $k$ (i.e., the travel time to block $k$ is greater than $\Delta t$ ). Recalling that $T_{1} \leq T_{2} \leq \Delta t$, Figure C.3 shows the only possible intersections of the lines defined by Equation C.18 with the shaded area shown in Figure C.2. 

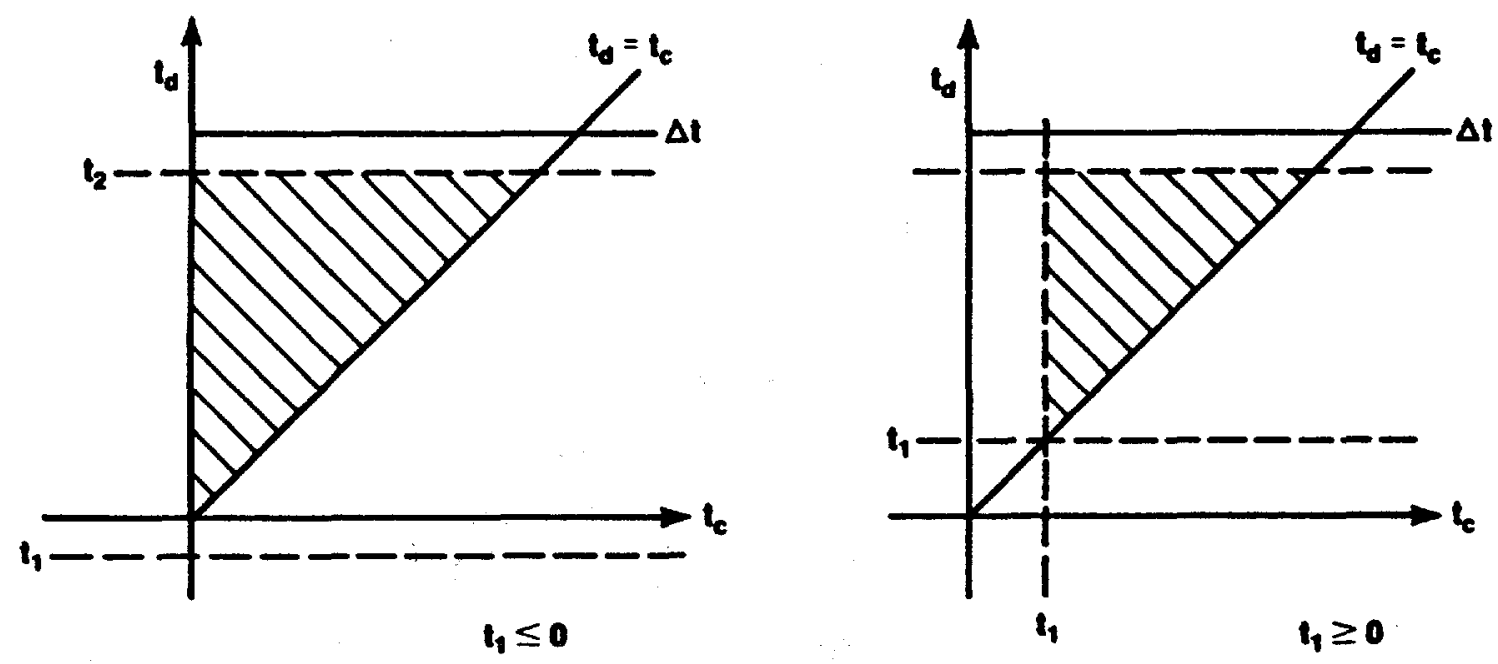

Figure C.3. Possible Integration Regions for the Case $v_{1}=v_{2}$

Condition $1 \quad\left(v_{1}=v_{2}\right)$ corresponding to Equation C.18 is examined first. If $\mathrm{T}_{2} \leq 0$, there is no contribution to grid block $\mathrm{k}$ (i.e., the travel time to block $k$ is greater than $\Delta t$ ). Recalling that $T_{1} \leq T_{2} \leq \Delta t$, Figure $C .3$ shows the only possible intersections of the lines defined by Equation C.18 with the shaded area shown in Figure C.2.

The shaded triangular regions have integration limits

$\left.\begin{array}{ll}\mathrm{T}_{\mathrm{d}_{1}}=0, & \mathrm{~T}_{\mathrm{c}_{1}}=0 \\ \mathrm{~T}_{\mathrm{d}_{2}}=\mathrm{t}_{2}, & \mathrm{~T}_{\mathrm{c}_{2}}=\mathrm{t}_{\mathrm{d}}\end{array}\right\}$ for $\mathrm{t}_{1} \leq 0$ 
$\left.\begin{array}{l}T_{d_{1}}=t_{1}, \quad T_{c_{1}}=t_{1} \\ T_{d_{2}}=t_{2}, \quad T_{c_{2}}=t_{d}\end{array}\right\}$ for $t_{1} \geq 0$

In either case, $\left(t_{1} \leq 0\right.$ or $\left.\left.t_{1} \geq\right)\right)$, Equation $C .14$ is solved as the sum of the integral evaluated over the triangular region and the integral evaluated over the rectangular region.

Condition $2\left(v_{1}>v_{2}\right)$ involves only Equation C.16 and is separated into the following subcases:

$$
\begin{array}{ll}
\text { Subcase 2.1 } & \mathrm{v}_{1}>\mathrm{v}_{2}>0 \\
\text { Subcase 2.2 } & \mathrm{v}_{1}>0>\mathrm{v}_{2} \\
\text { Subcase 2.3 } & 0>\mathrm{v}_{1}>\mathrm{v}_{2}
\end{array}
$$

Subcase 2.1 $\quad v_{1}>v_{2}>0$

If one writes $t_{d}=m t_{c}+B$ then

$$
\begin{gathered}
\mathrm{mt}_{\mathrm{c}}+\mathrm{B}_{1} \leq \mathrm{t}_{\mathrm{d}} \leq \mathrm{mt} \mathrm{t}_{\mathrm{c}}+\mathrm{B}_{2} \\
\text { for } \mathrm{m}=\frac{\mathrm{v}_{1}}{\mathrm{v}_{1}-\mathrm{v}_{2}}, \mathrm{~B}_{1}=\frac{\mathrm{k} \Delta \mathrm{x}-\mathrm{v}_{2} \Delta t}{\mathrm{v}_{1}-\mathrm{v}_{2}} \text {, and } \mathrm{B}_{2}=\frac{(\mathrm{k}+1) \Delta \mathrm{x}-\mathrm{v}_{2} \Delta t}{\mathrm{v}_{1}-\mathrm{v}_{2}}
\end{gathered}
$$

This subcase therefore has

$$
\begin{aligned}
& \mathrm{m}>1 \\
& \mathrm{~B}_{2}>\mathrm{B}_{1} \\
& \mathrm{~B}_{1}<\Delta t \\
& \mathrm{~T}_{1} *>\mathrm{T}_{2} * \\
& \mathrm{~T}_{2} *<\Delta t \\
& \mathrm{k} \geq 0
\end{aligned}
$$


where $\left(T_{1} *, T_{1} *\right)$ and $\left(T_{2} *, T_{2} *\right)$ are the points of intersection of lines $t_{d}=m t_{c}+B_{1}$, and $t_{d}=m t_{c}+B_{2}$ with line $t_{d}=t_{c}$, respectively. That is,

$$
\begin{aligned}
& \mathrm{T}_{1} *=\Delta \mathrm{t}-\frac{\mathrm{k} \Delta \mathrm{x}}{\mathrm{v}_{2}} \\
& \mathrm{~T}_{2} *=\Delta \mathrm{t}-\frac{(\mathrm{k}+1) \Delta \mathrm{x}}{\mathrm{v}_{2}}=\mathrm{T}_{1} *-\frac{\Delta \mathrm{x}}{\mathrm{v}_{2}}
\end{aligned}
$$

The restrictions $k \geq 0, \mathrm{~T}_{2} *<\Delta t, \mathrm{~T}_{1} *>\mathrm{T}_{2} *, \mathrm{~B}_{2}>\mathrm{B}_{1}$, and $\mathrm{m}>1$ follow directly from $v_{1}>v_{2}>0$. The restriction $B_{1}<\Delta t$ can be seen graphically to require a nonzero area of intersection. Algebraically, if

$$
B_{1} \geq \Delta t
$$

then

$$
\frac{k \Delta x-v_{2} \Delta t}{v_{1}-v_{2}} \geq \Delta t
$$

or

$$
k \Delta x \geq v_{1} \Delta t
$$

This implies that the distance to the nearest edge of grid block $k$ is longer than the distance a particle can possibly travel. That is, suppose a particle exits the cell at the earliest possible time, $t_{c}=0$. Then, migrating during the entire time $\Delta t$ at the fastest velocity, $v_{1}$, (which assumes $t_{d}=\Delta t$ ) results in the maximum distance $v_{1} \Delta t$.

Figure C.4 shows the 6 possible regions of integration for subcase 2.1 . The limits of integration for the subregions of each region are given in Table C.1. Lines $L_{1}$ and $L_{2}$ denote

$$
\begin{aligned}
& L_{1}: \quad t_{d}=m t_{c}+B_{1} \text { or } t_{c}=1 / m\left(t_{d}-B_{1}\right) . \\
& L_{2}: \quad t_{d}=m t_{c}+B_{2} \text { or } t_{c}=1 / m\left(t_{d}-B_{2}\right) .
\end{aligned}
$$



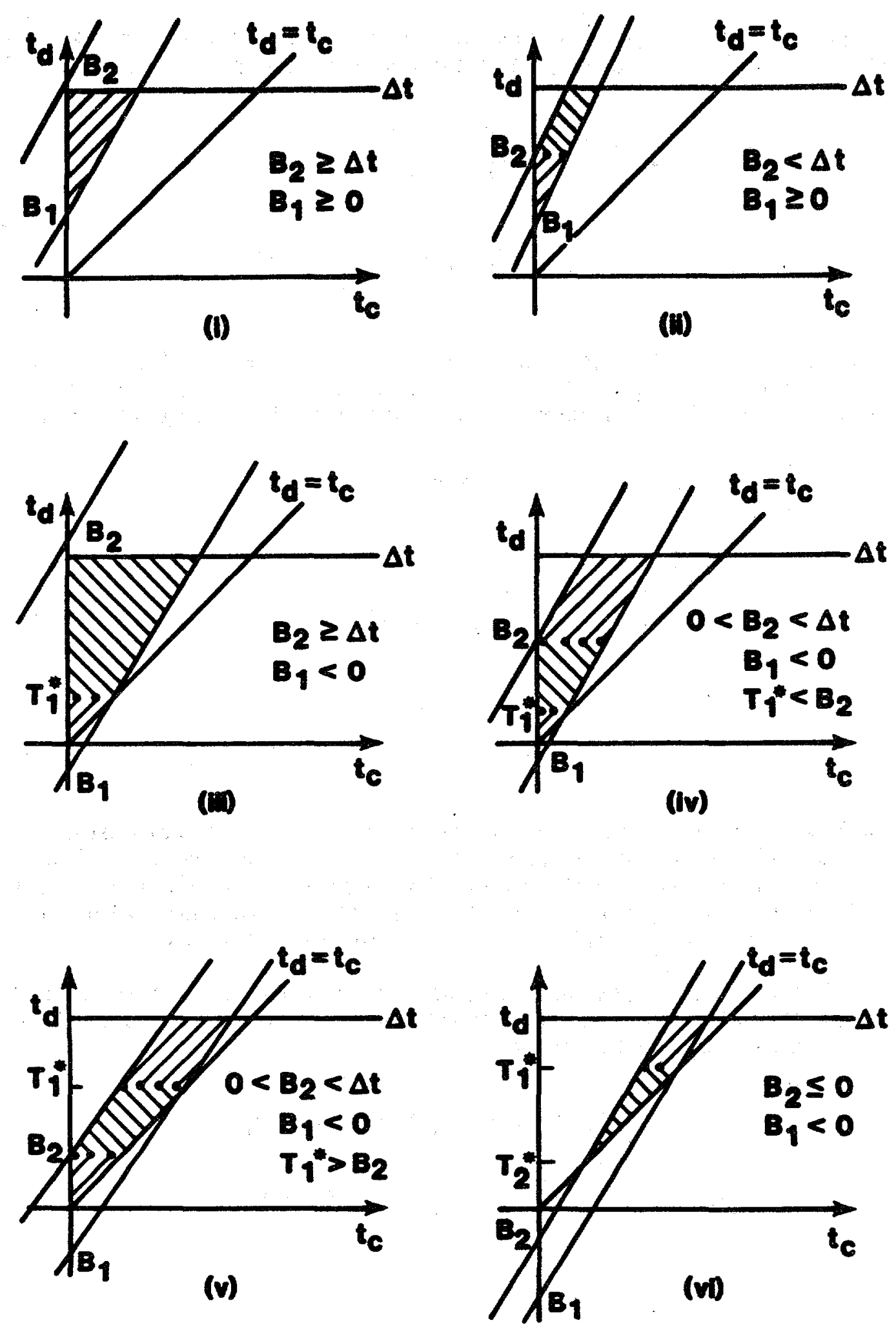

Figure C.4. The Possible Regions of Integration for Subcase 2.1 
Table C.1.

Integration Limits Needed to Evaluate Equation C.14

for Subcase 2.1

\begin{tabular}{|c|c|c|c|c|c|c|c|c|c|c|c|c|}
\hline \multirow[t]{2}{*}{ Graph } & \multicolumn{4}{|c|}{ Region 1} & \multicolumn{4}{|c|}{ Region 2} & \multicolumn{4}{|c|}{ Region 3} \\
\hline & $\mathrm{d}_{1}$ & $\mathrm{~T}_{2}$ & $\mathrm{~T}_{\mathrm{c}_{1}}$ & $\mathrm{~T}_{\mathrm{c}_{2}}$ & $\mathrm{~d}_{1}$ & $\mathrm{~T}_{2}$ & $\mathrm{~T}_{\mathrm{C}_{1}}$ & $\mathrm{~T}_{2}$ & $\mathrm{~d}_{1}$ & $\mathrm{~T}_{2}$ & $\mathrm{~T}_{\mathrm{c}_{1}}$ & $\mathrm{~T}_{\mathrm{c}_{2}}$ \\
\hline (i) & ${ }_{1}{ }_{1}$ & & 0 & $\mathrm{~L}_{1}$ & & $\mathrm{~N} / \mathrm{A}$ & & & & N/A & & \\
\hline (ii) & ${ }_{1}{ }_{1}$ & $\mathrm{~B}_{2}$ & 0 & $\mathrm{I}_{1}$ & $\mathrm{~B}_{2}$ & $\Delta t$ & $\mathrm{~L}_{2}$ & $\mathrm{I}_{1}$ & & N/A & & \\
\hline (iii) & 0 & $\mathrm{~T}_{1} *$ & 0 & $t_{d}$ & $\mathrm{~T}_{1}{ }^{*}$ & $\Delta t$ & 0 & $\mathrm{~L}_{1}$ & & N/A & & \\
\hline (iv) & 0 & $\mathrm{~T}_{1}{ }^{*}$ & 0 & $t_{d}$ & $\mathrm{~T}_{1}^{*}$ & $\mathrm{~B}_{2}$ & 0 & $\mathrm{~L}_{1}$ & ${ }_{2}$ & $\Delta t$ & $\mathrm{~L}_{2}$ & $\mathrm{~L}_{1}$ \\
\hline (v) & 0 & $\mathrm{~B}_{2}$ & 0 & ${ }^{t} \mathrm{~d}$ & $\mathrm{~B}_{2}$ & $\mathrm{~T}_{1} *$ & $\mathrm{~L}_{2}$ & $t_{d}$ & $\mathrm{~T}_{1}$ * & $\Delta t$ & $\mathrm{~L}_{2}$ & $\mathrm{~L}_{1}$ \\
\hline (vi) & $\mathrm{T}_{2}^{*}$ & $\mathrm{~T}_{1}{ }^{*}$ & $\mathrm{~L}_{2}$ & $t_{d}$ & $\mathrm{~T}_{1}{ }^{*}$ & $\Delta t$ & $\mathrm{~L}_{2}$ & $\mathrm{~L}_{1}$ & & N/A & & \\
\hline
\end{tabular}

The final integration for a region is a sum of integrals over its subregion.

Subcase $2.2 \quad v_{1}>0>v_{2}$

This subcase is restricted to

$$
\left.\begin{array}{l}
\left.\begin{array}{l}
0<\mathrm{m}<1 \\
\mathrm{~T}_{2}^{*}>\mathrm{T}_{1}^{*} \\
\mathrm{~T}_{1} * \Delta \mathrm{t} \\
\Delta t>\mathrm{B}_{1}>0
\end{array}\right\} \quad \mathrm{k} \geq 0 \\
\mathrm{~T}_{2} * \Delta t \\
\Delta t>\mathrm{B}_{2}>0
\end{array}\right\}
$$


$T_{1} *>\Delta t$ and $B_{1}>0$ follow from $v_{2}<0$ and $k \geq 0$. $T_{2} *<\Delta t$ and $B_{2}<\Delta t$ follow from $v_{2}<0$ and $k \leq-1$. Intercept $B_{1}$ is less than $\Delta t$ from the travel time required to the nearest edge of grid block $k$. Intercept $B_{2}$ is greater than zero for the same reason.

Figure C. 5 shows the 4 possible regions for subcase 2.2. Table C.2 lists the limits of integration by subregion.

Table C.2.

Integration Limits Needed to Evaluate Equation C.14

for Subcase 2.2

\begin{tabular}{|c|c|c|c|c|c|c|c|c|c|c|c|c|}
\hline \multirow[t]{2}{*}{ Graph } & \multicolumn{4}{|c|}{ Region 1} & \multicolumn{4}{|c|}{ Region 2} & \multicolumn{4}{|c|}{ Region 3} \\
\hline & $\mathrm{T}_{1}$ & $\mathrm{~d}_{2}$ & $\mathrm{~T}_{\mathrm{c}_{1}}$ & $\mathrm{~T}_{2}$ & $\mathrm{~d}_{1}$ & $\mathrm{~d}_{2}$ & $\mathrm{c}_{1}$ & ${ }_{c_{2}}^{T}$ & $\mathrm{~d}_{1}$ & $\mathrm{~d}_{2}$ & $\mathrm{~T}_{1}$ & $\mathrm{~T}_{\mathrm{c}_{2}}$ \\
\hline (i) & & $\Delta t$ & 0 & $t_{d}$ & & $\mathrm{~N} / \mathrm{A}$ & & & & $\mathrm{N} / \mathrm{A}$ & & \\
\hline (ii) & ${ }_{1}$ & $\mathrm{~B}_{2}$ & 0 & $\mathrm{~L}_{1}$ & ${ }_{2}$ & $\Delta t$ & $\mathrm{~L}_{2}$ & $\mathrm{~L}_{1}$ & & N/A & & \\
\hline (iii) & $\mathrm{B}_{1}$ & $\mathrm{~T}_{1}$ * & 0 & $\mathrm{~L}_{1}$ & $\mathrm{~T}_{1}{ }^{*}$ & $\mathrm{~B}_{2}$ & 0 & $t_{d}$ & $\mathrm{~B}_{2}$ & $\mathrm{~T}_{2}^{*}$ & $\mathrm{~L}_{2}$ & $\mathrm{t}_{\mathrm{d}}$ \\
\hline (iv) & 0 & $\mathrm{~B}_{2}$ & 0 & $t_{d}$ & ${ }_{2}$ & $\mathrm{~T}_{2}$ * & $\mathrm{L}_{2}$ & $t_{d}$ & & N/A & & \\
\hline
\end{tabular}

Subcase 2.3 $0>v_{1}>v_{2}$

SInce both velocities are negative, particles in these bins must travel in a negative direction. Thus, only $k \leq-1$ is allowed. The following restrictions apply.

$$
\begin{aligned}
& \mathrm{m}<0 \\
& \mathrm{~B}_{2}>\mathrm{B}_{1} \\
& \mathrm{~B}_{2}>0 \\
& \mathrm{~T}_{1} *<t \\
& \mathrm{~T}_{2} *<t
\end{aligned}
$$



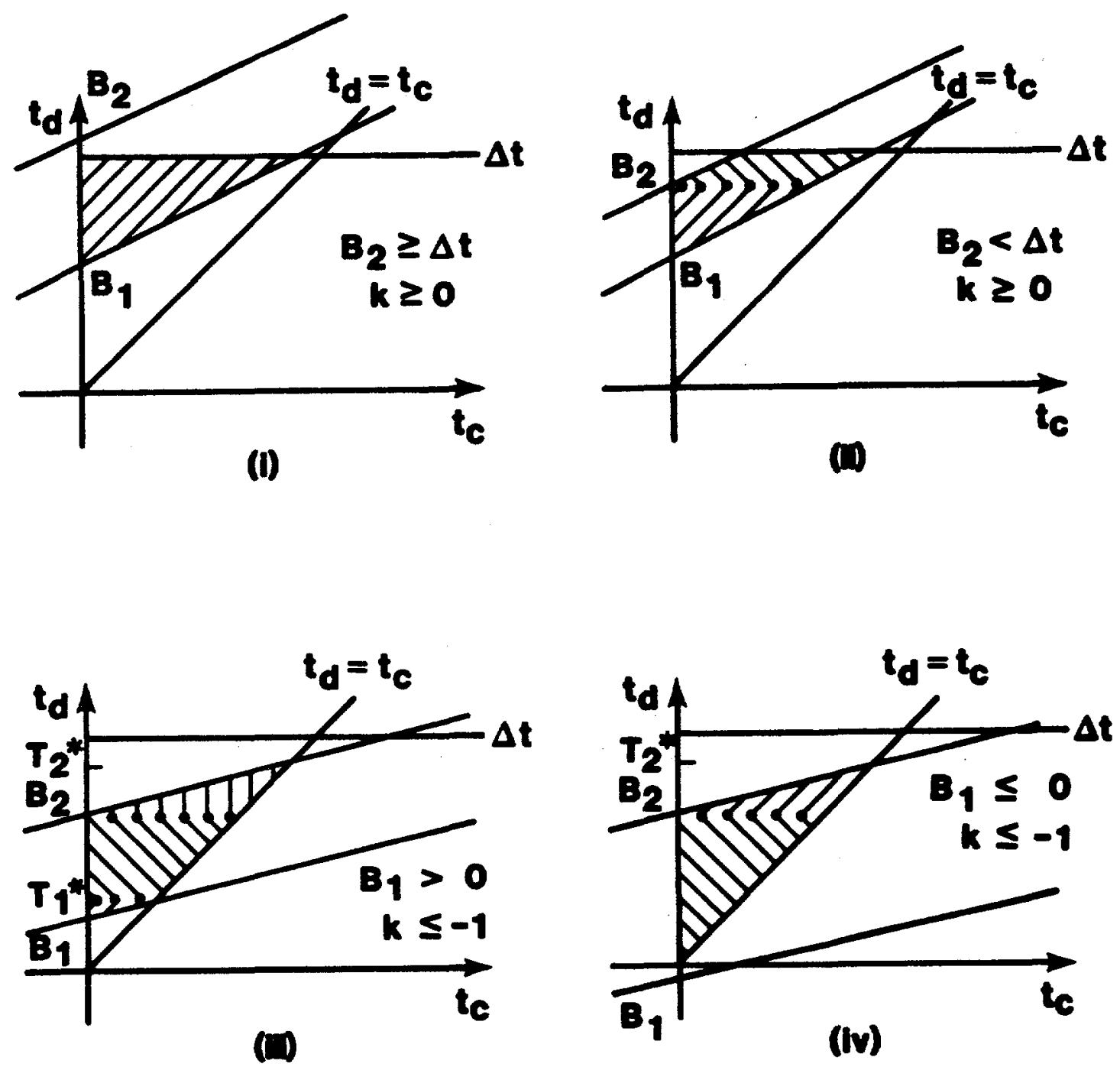

Figure C.5. Regions of Integration for Subcase 2.2 
$\mathrm{m}<0, \mathrm{~B}_{2}>\mathrm{B}_{1}, \mathrm{~T}_{1} *<\Delta t$, and $\mathrm{T}_{2} *<\Delta t$ follow from $\mathrm{k} \leq-1, \mathrm{v}_{2} \leq 0$, and $v_{1}-v_{2}>0$. Intercept $B_{2}$ is positive because the minimal travel time to the nearest edge of block $\mathrm{k}$ must be less than $\Delta t$.

Figure C.6 shows the 7 possible regions for subcase 2.3. Table C.3 lists the corresponding limits of integration by subregion.

The 17 regions of subcases 2.1 to 2.3 completely describe the integration limits for condition $2\left(v_{1}>v_{2}\right)$. The limits for condition $3\left(v_{2}>v_{1}\right)$ are the same if the roles of lines $L_{1}$ and $L_{2}$ are reversed. Thus, defining

$$
\begin{array}{ll}
\text { Subcase } 3.1 & \mathrm{v}_{2}>\mathrm{v}_{1}>0 \\
\text { Subcase } 3.2 & \mathrm{v}_{2}>0>\mathrm{v}_{1} \\
\text { Subcase 3.3 } & 0>\mathrm{v}_{2}>\mathrm{v}_{1}
\end{array}
$$

one has the correspondences

$$
\begin{array}{lll}
\text { Subcase } 3.1 & \leftrightarrow & \text { Subcase } 2.3 \\
\text { Subcase } 3.2(k \geq 0) & \leftrightarrow & \text { Subcase } 2.2(k \leq-1) \\
\text { Subcase } 3.2(k \leq-1) & \leftrightarrow & \text { Subcase } 2.2(k \geq 0) \\
\text { Subcase } 3.3 & \leftrightarrow & \text { Subcase } 2.1
\end{array}
$$

Returning to Equation C.14, it can be rewritten as

$$
\begin{aligned}
& \operatorname{pr}(2,1, k, b)=k_{a} \int_{T_{d_{1}}}^{T_{d_{2}}} e^{-\left(\lambda_{1}-\lambda_{2}\right) t_{d}}\left[\int_{T_{c_{1}}}^{T_{c}} e^{\lambda_{1} t_{c}} d t_{c}\right] d t_{d} \\
& =\frac{K_{a}}{\lambda_{1}} \int_{T_{1}}^{T_{d_{2}}} e^{-\left(\lambda_{1}-\lambda_{2}\right) t_{d}}\left[e^{\lambda_{1} T_{c_{2}}}-e^{\lambda_{1} T_{c}}\right] d t_{d}
\end{aligned}
$$



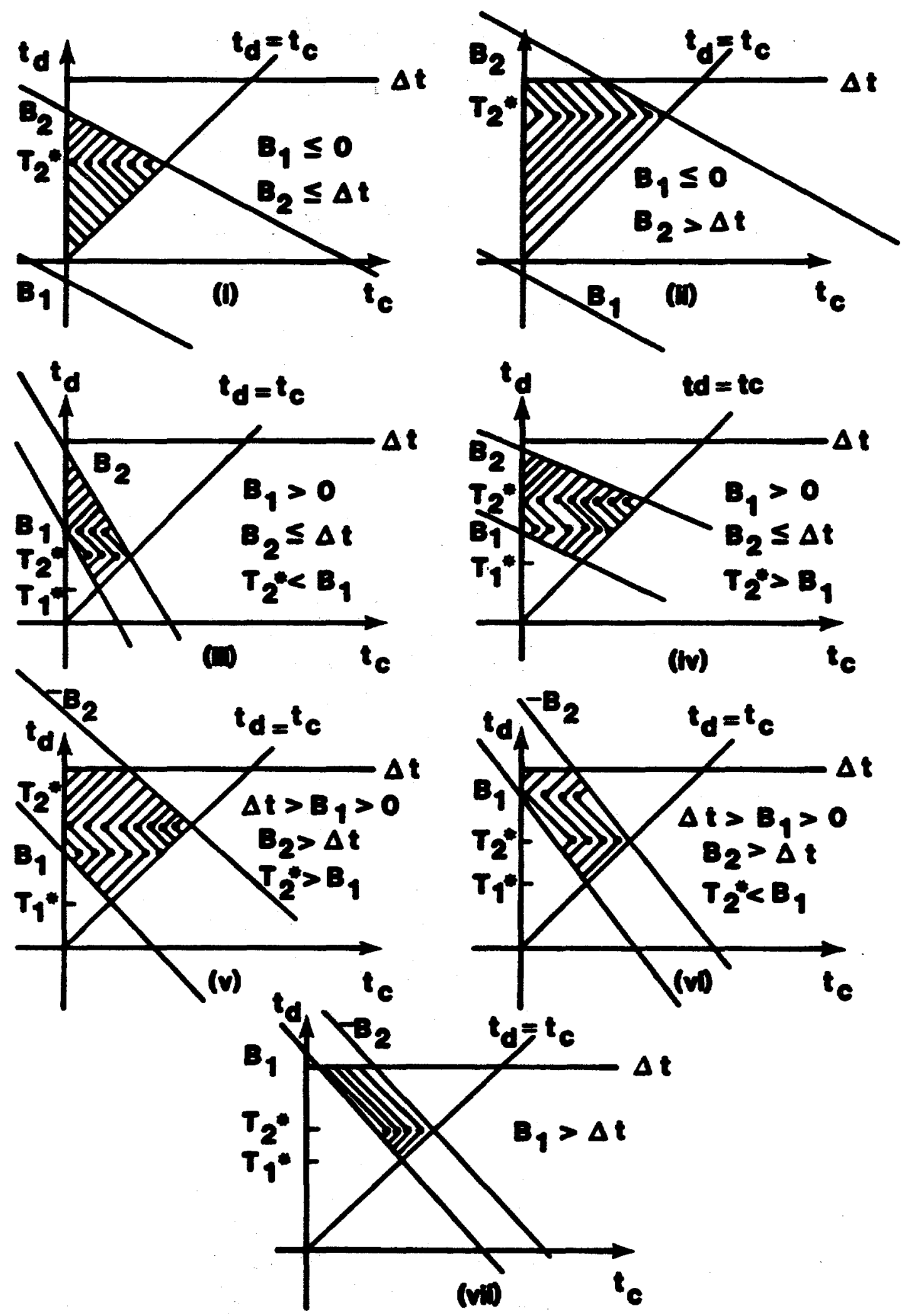

Figure C.6. Regions of Integration for Subcase 2.3 
Table C.3.

Integration Limits Needed to Evaluate Equation C.14 for Subcase 2.3

\begin{tabular}{|c|c|c|c|c|c|c|c|c|c|c|c|c|}
\hline \multirow[t]{2}{*}{ Graph } & \multicolumn{4}{|c|}{ Region 1} & \multicolumn{4}{|c|}{ Region 2} & \multicolumn{4}{|c|}{ Region 3} \\
\hline & $\mathrm{d}_{1}$ & $\mathrm{~T}_{2}$ & $c_{1}$ & $\mathrm{~T}_{2}$ & $\mathrm{~T}_{1}$ & $\mathrm{~T}_{2}$ & $\mathrm{~T}_{\mathrm{c}_{1}}$ & $\mathrm{~T}_{2}$ & $\mathrm{~T}_{1}$ & $\mathrm{~d}_{2}$ & $\mathrm{~T}_{1}$ & $\mathrm{~T}_{2}$ \\
\hline (i) & 0 & $\mathrm{~T}_{2}{ }^{*}$ & 0 & $t_{d}$ & $\mathrm{~T}_{2}{ }^{*}$ & $\mathrm{~B}_{2}$ & 0 & $\mathrm{~L}_{2}$ & & N/A & & \\
\hline (ii) & 0 & $\mathrm{~T}_{2}{ }^{*}$ & 0 & $t_{d}$ & $\mathrm{~T}_{2}{ }^{*}$ & $\Delta t$ & 0 & $\mathrm{~L}_{2}$ & & N/A & & \\
\hline (iii) & $\mathrm{T}_{1}{ }^{*}$ & $\mathrm{~T}_{2}{ }^{*}$ & $\mathrm{~L}_{1}$ & $t_{d}$ & $\mathrm{~T}_{2}{ }^{*}$ & $B_{1}$ & $\mathrm{~L}_{1}$ & $\mathrm{~L}_{2}$ & $\mathrm{~B}_{1}$ & $\mathrm{~B}_{2}$ & 0 & $\mathrm{~L}_{2}$ \\
\hline (iv) & $\mathrm{T}_{1}$ * & $\mathrm{B}_{1}$ & $\mathrm{~L}_{1}$ & $t_{d}$ & $B_{1}$ & $\mathrm{~T}_{2}{ }^{*}$ & 0 & $t_{d}$ & $\mathrm{~T}_{2}{ }^{*}$ & $\mathrm{~B}_{2}$ & 0 & $\mathrm{~L}_{2}$ \\
\hline (v) & $\mathrm{T}_{1}{ }^{*}$ & ${ }_{1}$ & $\mathrm{~L}_{1}$ & $t_{d}$ & ${ }_{1}$ & $\mathrm{~T}_{2}$ * & 0 & $t_{d}$ & $\mathrm{~T}_{2}$ * & $\Delta t$ & 0 & $\mathrm{~L}_{2}$ \\
\hline (vi) & $\mathbf{T}_{1}{ }^{*}$ & $\mathrm{~T}_{2}{ }^{*}$ & $\mathrm{~L}_{1}$ & ${ }^{t} d$ & $\mathrm{~T}_{2}{ }^{*}$ & ${ }_{1}$ & $\mathrm{~L}_{1}$ & $\mathrm{~L}_{2}$ & $\mathrm{~B}_{1}$ & $\Delta t$ & 0 & $\mathrm{~L}_{2}$ \\
\hline (vii) & $\mathrm{T}_{1}{ }^{*}$ & $\mathrm{~T}_{2}{ }^{*}$ & $\mathrm{~L}_{1}$ & $t_{d}$ & $\mathrm{~T}_{2}{ }^{*}$ & $\Delta t$ & $\mathrm{~L}_{1}$ & $\mathrm{~L}_{2}$ & & $N / A$ & & \\
\hline
\end{tabular}

where $\mathrm{K}_{\mathrm{a}}=\frac{\mathrm{h}_{1,2} \lambda_{1} \mathrm{e}^{-\lambda_{2} \Delta t}}{\Delta \mathrm{tN}} \mathrm{v}$. From the above conditions, it is known that $T_{c_{1}}$ and $T_{c_{2}}$ are linear functions of $t_{d}$. In general, one can write

$$
\begin{aligned}
& \mathrm{T}_{c_{1}}=\alpha_{1} t_{d}-\beta_{1} \\
& \mathrm{~T}_{c_{2}}=\alpha_{2} t_{d}-\beta_{2}
\end{aligned}
$$


Equation C. 27 becomes

$$
\begin{aligned}
& \operatorname{pr}(2,1, b, k)=\frac{k_{a}}{\lambda_{1}} \int_{T_{d_{1}}}^{T_{d_{2}}}\left[e^{-\left(\lambda_{1}\left(1-a_{2}\right)-\lambda_{2}\right) t_{d}} e^{-\lambda_{1} \beta_{2}}\right. \\
& \left.e^{-\left(\lambda_{1}\left(1-\alpha_{1}\right)-\lambda_{2}\right) t_{d}} e^{-\lambda_{1} \beta_{1}}\right] d t_{d} \\
& =\frac{\mathrm{k}_{\mathrm{a}}}{\lambda_{1}} \mathrm{e}^{-\lambda_{1} \beta_{2}}\left[\frac{\mathrm{e}^{-\left(\lambda_{1}\left(1-\alpha_{2}\right)-\lambda_{2}\right) \mathrm{T}_{\mathrm{d}_{1}}-\left(\lambda_{1}\left(1-\alpha_{2}\right)-\lambda_{2}\right) \mathrm{T}_{\mathrm{d}}}}{\lambda_{1}\left(1-\alpha_{2}\right)-\lambda_{2}}\right] \\
& -e^{-\lambda_{1} \beta_{1}}\left[\frac{\mathrm{e}^{-\left(\lambda_{1}\left(1-\alpha_{1}\right)-\lambda_{2}\right) \mathrm{T}_{\mathrm{d}_{1}}-\left(\lambda_{1}\left(1-\alpha_{1}\right)-\lambda_{2}\right) \mathrm{T}_{\mathrm{d}}}}{\lambda_{1}\left(1-\alpha_{1}\right)-\lambda_{2}}\right]
\end{aligned}
$$

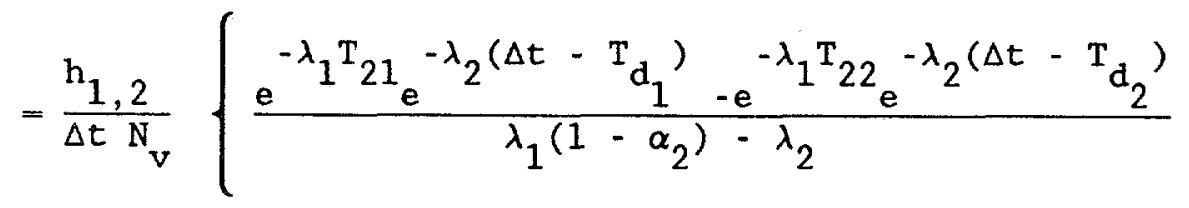

$$
\begin{aligned}
& \left.-\frac{e^{-\lambda_{1} T_{11} e^{-\lambda_{2}\left(\Delta t-T_{d_{1}}\right)}-e^{-\lambda_{1} T_{12}} e^{-\lambda_{2}\left(\Delta t-T_{d_{2}}\right)}}}{\lambda_{1}\left(1-\alpha_{1}\right)-\lambda_{2}}\right),
\end{aligned}
$$


where

$$
\mathrm{T}_{\mathrm{rp}}=\left(1-\alpha_{\mathrm{r}}\right) \mathrm{T}_{\mathrm{d}}+\beta_{\mathrm{r}} \quad \mathrm{r}, \mathrm{p}=1,2 .
$$

The above integration is valid as long as $\lambda_{1}\left(1-\alpha_{r}\right)-\lambda_{2} \neq 0$. The possible values for $\alpha_{r}$ are

$$
\left.\begin{array}{l}
\alpha_{r}=0 \\
\alpha_{r}=1 \\
\alpha_{r}=1 / m=\frac{v_{1}-v_{2}}{v_{1}}
\end{array}\right\}
$$

If $\alpha_{r}=0, \lambda_{1} \neq \lambda_{2}$ is required; If $\alpha_{r}=1, \lambda_{2} \neq 0$; and if $\alpha_{r}=1 / \mathrm{m}$.

$$
\frac{\lambda_{1}}{\lambda_{2}} \neq \frac{v_{1}}{v_{2}}
$$

If $\lambda_{1}\left(1-\alpha_{r}\right)-\lambda_{2}=0$ for any case. Equation C.29 is replaced by

$$
\operatorname{pr}(2,1, \mathrm{~b}, \mathrm{k})=\frac{\mathrm{h}_{1,2}}{\Delta \mathrm{t} \mathrm{N}_{\mathrm{v}}} \mathrm{e}^{-\lambda_{1} \beta_{\mathrm{r}}} \mathrm{e}^{-\lambda 2^{\Delta t}\left(\mathrm{~T}_{\mathrm{d}_{2}}-\mathrm{T}_{\mathrm{d}_{1}}\right)}
$$

Finally, $T_{x p} \geq 0$ since

$$
t_{c}=\alpha_{r} t_{d}-\beta_{r}
$$

implies

$$
t_{d}-t_{c}=\left(1-\alpha_{r}\right) t_{d}+\beta_{r}
$$

for all values of $t_{d}$, and the left hand side of Equation C.32 is nonnegative. In fact

$$
T_{r p}=T_{d p}-T_{c r} ; r, p=1,2
$$


The final step in finding $S_{F}$ is to sum over velocity bins for each block $k$,

$$
S_{F}(2,1, k)=\sum_{b=1}^{N_{v}} \quad \operatorname{pr}(2,1, k, b)
$$

Here, $\operatorname{pr}(2,1, k, b)=0$ if

$$
\begin{aligned}
& \Delta t \max \left\{v_{1}^{b}, v_{2}^{b}\right\} \leq k \Delta x ; k \geq 0 \\
& \Delta t \min \left\{v_{1}^{b}, v_{2}^{n}\right\} \geq(k+1) \Delta x ; k \leq-1 .
\end{aligned}
$$

In practice, the entries $S_{F}(2,1, k)$ may be positive but negligible for some extreme values of $k$.

The last case $(j>1)$ involves decay chains of length greater than two. To implement the algorithm, a simplifying assumption is made. For the chain

$$
i-P_{i} \rightarrow i-P_{i}+1 \rightarrow \ldots \rightarrow i-i \rightarrow i,
$$

if a source particle of species type $i-j$ (for some $j, 2 \leq j \leq p_{i}$ ) crosses the cell boundary at $t_{c}$ and is of species type $i$ at the end of $\Delta t$, the decay from species $i-j$ to species $i-1$ is assumed to be a single process. During this decay process, the particle travels with a velocity that is an arithmetic average of the velocities assigned to species $i-j, \ldots \ldots i-1$.

The average velocity for the velocity bin $b$ is

$$
v^{b}=\frac{i}{j} \sum_{j}^{j=1} v_{i-j^{\prime}}^{b}
$$

There are five probabilities similar to the case $j=1$.

1. The probability that an atom of species $i-j$ exits the cell during time $d t_{c}$ about $t_{c}$ is

$$
d t_{c} / \Delta t
$$


2. The probability that an atom of species $i-j$ decays to species $i-1$ during time $t_{d}-t_{c}$ is

$$
\begin{aligned}
& \sum_{n=2}^{j} h_{i-n ; i-n+1} \lambda_{i-n_{l=2}} \sum_{\sum}^{j} \frac{e^{-\lambda_{i-\ell}\left(t_{d}-t_{c}\right)}-e^{-\lambda_{i-1}\left(t_{d}-t_{c}\right)}}{\underset{\Pi}{j}\left(\lambda_{i-m}-\lambda_{i-l}\right)} \\
& \mathrm{m}-1 \\
& \mathrm{~m} \neq \ell
\end{aligned}
$$

3. The probability that an atom of species $i-1$ decays to species $i$ during $d t_{d}$ about $t_{d}$ is

$$
h_{i-1, i} \lambda_{i-1} d t_{d}
$$

4. The probability that an atom species $i$ survives decay from $t_{d}$ to $\Delta t$ is

$$
e^{-\lambda_{i}\left(\Delta t-t_{d}\right)}
$$

5. The probability that an atom is assigned to velocity bin $b$ is $1 / \mathrm{N}_{\mathrm{v}}$. Similar to $\mathrm{j}=1$, it is assumed that the particles maintain the same velocity bin over $\Delta t$ as they decay.

The probability that a source particle of type $i-j$ ends $\Delta t$ occupying grid block $k$ as type $i$ is

$$
\operatorname{pr}(i-j, i, k, b)=\frac{h_{i-1, i} i_{i-1}}{N_{v} \Delta t} \prod_{n=2}^{j} h_{i-n, i-n+1} g_{i-n} e^{-\lambda \Delta t}
$$

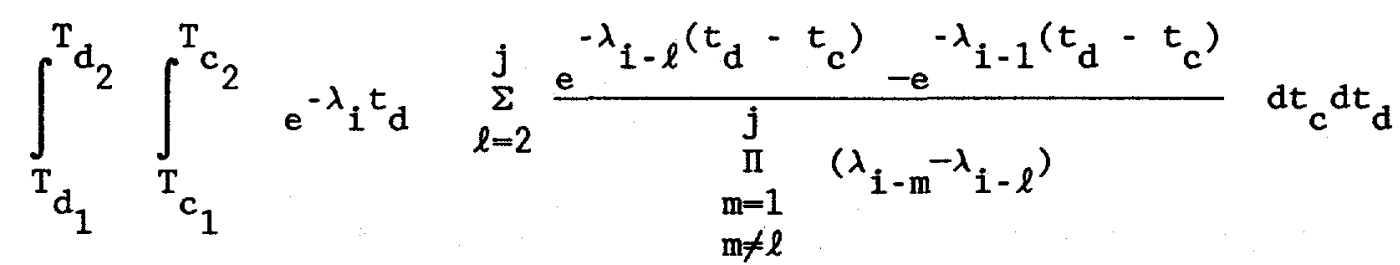

where $T_{d_{1}}, T_{d_{2}}, T_{c_{1}}, T_{c_{2}}$ are determined as in the case $j=1$ with $v_{1}$

replacing $v_{2}$ and $\vec{v}^{b}$ (Equation c.36) replacing $v_{1}$. In particular, recall that $t_{c}$ is a linear function of $t_{d}$ and 


$$
\begin{aligned}
& \mathrm{T}_{\mathrm{c}_{1}}=\alpha_{1} \mathrm{t}_{\mathrm{d}}-\beta_{1} \\
& \mathrm{~T}_{\mathrm{c}_{2}}=\alpha_{2} \mathrm{t}_{\mathrm{d}}-\beta_{2}
\end{aligned}
$$

The details of solving Equation C.37 are omitted except for noting the use of the identity

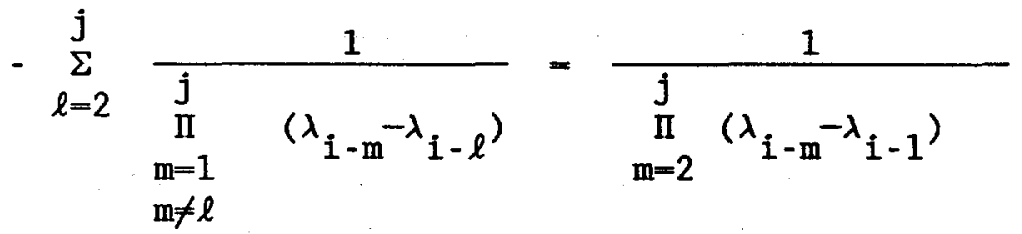

in order to combine terms following the integration.

Thus,

$$
\begin{aligned}
& \operatorname{pr}(i-j, i, k, b)=\frac{1}{N_{v} \Delta t} \prod_{n=1}^{j} h_{i-n, i-n+1} \lambda_{i-n}
\end{aligned}
$$

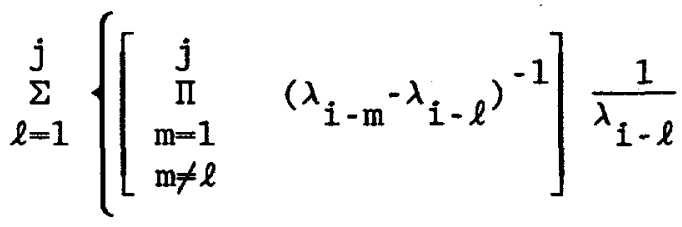

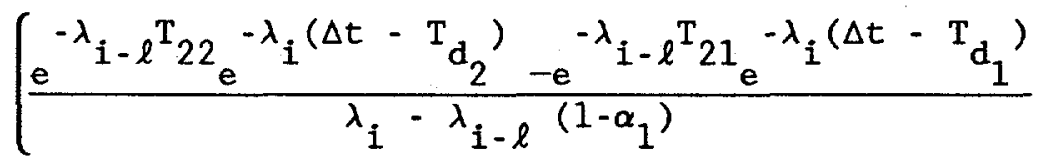

$$
\begin{aligned}
& \left.\left.-\frac{e^{-\lambda i-\ell{ }^{T} 12} e^{-\lambda_{i}\left(\Delta t-T_{d_{2}}\right)}-e^{-\lambda_{i-\ell} T_{11}} e^{-\lambda_{i}\left(\Delta t-T_{d_{1}}\right)}}{\lambda_{i}-\lambda_{i-\ell}\left(1-\alpha_{1}\right)}\right)\right]
\end{aligned}
$$

where the $\mathrm{T}_{\mathrm{rp}}(\mathrm{r}, \mathrm{p}=1,2)$ are defined by Equation $\mathrm{c} .30$. The restrictions of nonzero denominators still apply and if zero denominators are detected the term 


$$
e^{-\lambda i^{\Delta t}} e^{-\lambda i-\ell{ }_{r}}\left(T_{d_{2}}-T_{d_{1}}\right)
$$

replaces

$$
\frac{e^{-\lambda} i-\ell^{T} r p e^{-\lambda}\left(\Delta t-T_{d p}\right)}{\lambda_{i}-\lambda_{i-l}\left(1-\alpha_{r}\right)}
$$

The conditions stated in Equation $C .35$ apply to set $\operatorname{pr}(i-j, i, k, b)=0$ for blocks $k$ that do not receive source particles. Collecting fractions over velocity intervals then yields

$$
S_{F}(i, i-j, k)=\sum_{b=1}^{N_{v}} \operatorname{pr}(i-j, i, k, b)
$$

Equation C.40 must be generated for each parent $i-j, j=2, \ldots, P_{1}$. Finally, defining

$$
\prod_{\substack{m=1 \\ m \neq l}}^{j}\left(\lambda_{i-m}-\lambda_{i-\ell}\right)=1
$$

for the case $\ell=j=1$, Equation C. 39 will contain Equation C. 29. Thus, Equations $C .13$ and $C .40$ can be used to completely define $S_{F}$. 
The storage of decay chain information in NEFTRAN II is presented in this appendix, first for the case where no branches occur in the decay chain, and next for the case where branches are present in the chain. The subroutine BRANCH is then discussed in terms of arrays presented in the first two discussions.

I. Decay Chain Without Branching.

$$
\begin{aligned}
& \text { Example: } 1 \rightarrow 2 \rightarrow 3 \rightarrow 4 \rightarrow 5 \\
& \text { NEFTRAN potentially transports } \frac{5(5+1)}{2}=15 \\
& \begin{array}{ll}
1.1 \rightarrow 1 & 8.3 \rightarrow 4 \\
2.2 \rightarrow 2 & 9.2 \rightarrow 3 \rightarrow 4 \\
3.1 \rightarrow 2 & 10.1 \rightarrow 2+3 \rightarrow 4 \\
4.3 \rightarrow 3 & 11.5 \rightarrow 5 \\
5.2 \rightarrow 3 & 12.4 \rightarrow 5 \\
6.1 \rightarrow 2 \rightarrow 3 & 13.3 \rightarrow 4 \rightarrow 5 \\
7.4 \rightarrow 4 & 14.2 \rightarrow 3 \rightarrow 4 \rightarrow 5 \\
& 15.1 \rightarrow 2 \rightarrow 3+4 \rightarrow 5
\end{array}
\end{aligned}
$$

However, Subroutine BRANCH may determine that some of the longer subchains are not necessary. That is, the contribution to species 5 from the decay of species 1 over a time step $\Delta t$, may be insignificant.

If BRANCH decides that all are necessary, then define the LPS (loop start) and LPE (loop end) arrays for each species as follows:

$$
\begin{array}{ll}
\operatorname{LPS}(1)=1 & \operatorname{LPE}(1)=1 \\
\operatorname{LPS}(2)=2 & \operatorname{LPE}(2)=3 \\
\operatorname{LPS}(3)=4 & \operatorname{LPE}(3)=6 \\
\operatorname{LPS}(4)=7 & \operatorname{LPE}(4)=10 \\
\operatorname{LPS}(5)=11 & \operatorname{LPE}(5)=15
\end{array}
$$

If the subchains above were numbered $1-15$, then those ending with species I are found from

$$
\text { LPS (I) to } \operatorname{LPE}(I) \text {. }
$$

The arrays LPS and LPE are used to define end points of DO-loops.

$$
\begin{gathered}
\text { DO } 20 \mathrm{I}=1, \text { NOISO } \\
\mathrm{J} 1=\mathrm{LPS}(\mathrm{I}) \\
\mathrm{J} 2=\mathrm{LPE}(\mathrm{I}) \\
\text { DO } 10 \mathrm{~J}=\mathrm{J} 1, \mathrm{~J} 2
\end{gathered}
$$


This type of loop occurs throughout NEFTRAN II.

In such a loop, variable $\mathrm{J}$ is normally used to retrieve information about subchain $J$. Subchains are defined by the LRP-array.

$$
\begin{aligned}
& \operatorname{LRP}(1,1)=1 \\
& \operatorname{LRP}(1,2)=1 \\
& \operatorname{LRP}(2,1)=2 \\
& \operatorname{LRP}(2,2)=1 \\
& \operatorname{LRP}(3,1)=1 \\
& \operatorname{LRP}(3,2)=2 \\
& \operatorname{LRP}(4,1)=3 \\
& \operatorname{LRP}(4,2)=1 \\
& \operatorname{LRP}(5,1)=2 \\
& \operatorname{LRP}(5,2)=2 \\
& \operatorname{LRP}(6,1)=1 \\
& \operatorname{LRP}(6,2)=3
\end{aligned}
$$

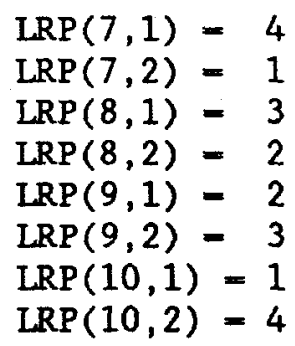

For species I, look in LRP array from

$$
\operatorname{LPS}(I) \text { to } \operatorname{LPE}(I) \text {. }
$$

For $\operatorname{LPS}(\mathrm{I}) \leq \mathrm{J} \leq \operatorname{LPE}(\mathrm{I})$, subchain $\mathrm{J}$ has $\operatorname{LRP}(\mathrm{J}, 1)$ members and the first member is species $\operatorname{LRP}(\mathrm{J}, 1)$.

Example: $I=3,4 \leq \mathrm{J} \leq 6$. If $J=6$, then subchain 6 has length $\operatorname{LRP}(6,2)=3$ and the top member of the chain is $\operatorname{LRP}(6,1)=1$.

If branches were not allowed, only one column (either one) of LRP would be necessary. In fact, LRP could probably be done away with. Its necessity will be demonstrated in Section II.

Application. Consider the DP-array, which contains decay and production fractions for each subchain over a time step. That is,

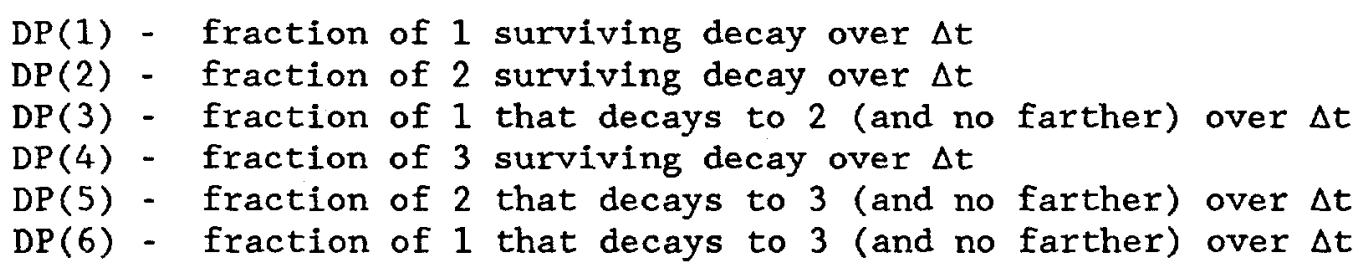

So, to update an inventory for species 3 , treat all subchains ending in 3 , which can be found between LPS(3) and LPE(3).

$$
\begin{aligned}
& \mathrm{SUM}=0 \\
& \mathrm{JI}=\operatorname{LPS}(3) \\
& \mathrm{J} 2=\operatorname{LPE}(3)
\end{aligned}
$$




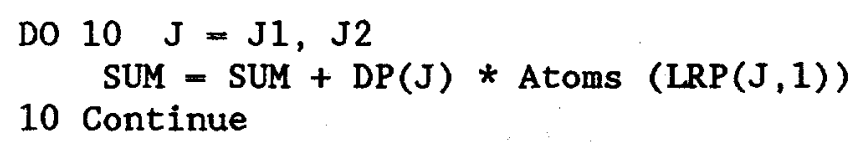

Variable SUM will contain the inventory of species 3 after a time step $\Delta t$, where the atoms array contains inventories of all species at time 0 .

There are several other arrays in NEFTRAN which rely on the pointers LPS and LPE. All such arrays are dimensioned to the maximum number of subchains ( +1 for some).

$$
(B, J V, V, N, J F, J S F, \text { INSR, JPSC) }
$$

II. Decay Chain With Branching.

Example:

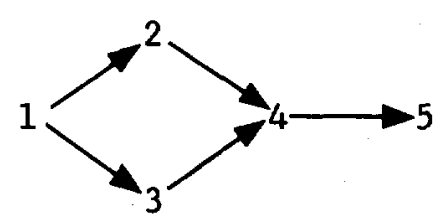

The possible subchains are:

$$
\begin{aligned}
& \text { 1. } 1 \rightarrow 1 \\
& \text { 2. } 2 \rightarrow 2 \\
& \text { 3. } 1 \rightarrow 2 \\
& \text { 4. } 3 \rightarrow 3 \\
& \text { 5. } 1 \rightarrow 3 \\
& 6 . \quad 4 \rightarrow 4 \\
& 7 . \quad 2 \rightarrow 4 \\
& \text { 8. } 1 \rightarrow 2 \rightarrow 4
\end{aligned}
$$

LRP Col. 1

$$
\begin{aligned}
& 9 . \quad 3 \rightarrow 4 \\
& 10 . \quad 1 \rightarrow 3 \rightarrow 4 \\
& 11 . \quad 5 \rightarrow 5 \\
& 12 . \quad 4 \rightarrow 5 \\
& 13 . \quad 2 \rightarrow 4 \rightarrow 5 \\
& 14 . \quad 1 \rightarrow 2 \rightarrow 4 \rightarrow 5 \\
& 15 . \quad 3 \rightarrow 4 \rightarrow 5 \\
& 16 . \quad 1 \rightarrow 3 \rightarrow 4 \rightarrow 5
\end{aligned}
$$

LRP Col, 2

$\begin{array}{llll}\text { row } 1 & 1 & 1 & \operatorname{LPS}(1)=1, \operatorname{LPE}(1)=1 \\ \text { row } 2 & 2 & 1 & \operatorname{LPS}(2)=2, \operatorname{LPE}(2)=3 \\ \text { row } 3 & 1 & 2 & \\ \text { row } 4 & 3 & 1 & \operatorname{LPS}(3)=4, \operatorname{LPE}(3)=5 \\ \text { row } 5 & 1 & 2 & \\ \text { row } 6 & 4 & 1 & \operatorname{LPS}(4)=6, \operatorname{LPE}(4)=10 \\ \text { row } 7 & 2 & 2 & \\ \text { row } 8 & 1 & 2 & \\ \text { row } 9 & 3 & 3 & \\ \text { row } 10 & 1 & 1 & \operatorname{LPS}(5)=11, \operatorname{LPE}(5)=16 \\ \text { row } 11 & 5 & 2 & \end{array}$


row 13

row 14

row 15

row 16

Note that row 8 is the same as row 10 and row 14 is the same as row 16 . However, the information in row 8 in arrays $B, J V, \ldots$ is for subchain $\# 8$, not \#10.

There are places in the code, however, where this does present a problem. For example, if the subchain ends in species 4, is of length 3 , and has ultimate parent 1 ; what is the identity of the intermediate species?

$$
1 \rightarrow 2 \rightarrow 4 \text { or } 1+3 \rightarrow 4 \text { ? }
$$

The intermediate species identity must be known for finding discharge factors. Two velocities are required $v_{4} \& v_{1 \rightarrow 2}$ or $v_{4} \& v_{1 \rightarrow 3}$

Examining subroutine SETDIS:

$$
\begin{aligned}
& 1 \rightarrow 2 \rightarrow 4 \quad \text { ISV }=2 \\
& \mathrm{KP}=8 \quad \text { ISP }=1 \\
& \text { find } 1 \rightarrow 2 \text {, } \\
& \mathrm{IJ}=\operatorname{LPS}(2), \operatorname{LPE}(3)-2,3 \\
& \text { ISL }=L T-1=2 \\
& \mathrm{KS}=3 \\
& 1 \rightarrow 3 \rightarrow 4 \\
& \text { ISV }=3 \\
& \mathrm{KP}=10 \\
& I S P=1 \\
& I J=4,5 \\
& \text { find } 1 \rightarrow 3 \\
& \text { ISL }=2 \\
& 4 \rightarrow 5 \\
& I S V=4 \\
& 2+4 \rightarrow 5 \\
& \mathrm{KP}=13 \\
& I J=6,10 \\
& \text { find } 2 \rightarrow 4 \\
& \text { ISP }=2 \\
& \text { KS }=6 \\
& \text { ISL }=2 \\
& 1 \rightarrow 2 \rightarrow 4 \rightarrow 5 \\
& I J=8,10 \\
& \mathrm{KP}=14 \\
& \text { find } 1 \rightarrow 2 \rightarrow 4 \\
& \text { ISP }-1 \\
& \mathrm{KS}=8 \\
& 3 \rightarrow 4+5 \\
& I J=9,10 \\
& \text { find } 3 \rightarrow 4 \\
& \text { ISP }=3 \\
& \mathrm{KS}=9 \\
& 1 \rightarrow 3 \rightarrow 4 \rightarrow 5 \\
& I J=10,10 \\
& \text { ISP }=1 \\
& \mathrm{KP}=16 \\
& \text { find } 1 \rightarrow 3 \rightarrow 4 \\
& \mathrm{KS}=10 \\
& I S L=3 \\
& I S L=2 \\
& I S L=3
\end{aligned}
$$

Treating/Locating Branching Fractions

$$
\begin{aligned}
& 1 \rightarrow 2 \text { at } .3 \\
& 1 \rightarrow 3 \text { at } .7
\end{aligned}
$$


$\begin{array}{llll}\text { On input: } & \text { Iso } 2 & \text { par }=1 & \text { frac }=.3 \\ & \text { Iso } 3 & \text { par }=1 & \text { frac }=.7\end{array}$

In CHAIN :

IFR $\quad$ BRFR

$\begin{array}{lll}1 . & 973 & 9 . .7 .7 \\ 2 . & 0 & 0 . \\ 3 . & 0 & 0 . \\ 4 . & 0 & 0 . \\ 5 . & 0 & 0 .\end{array}$

In SETDIS :

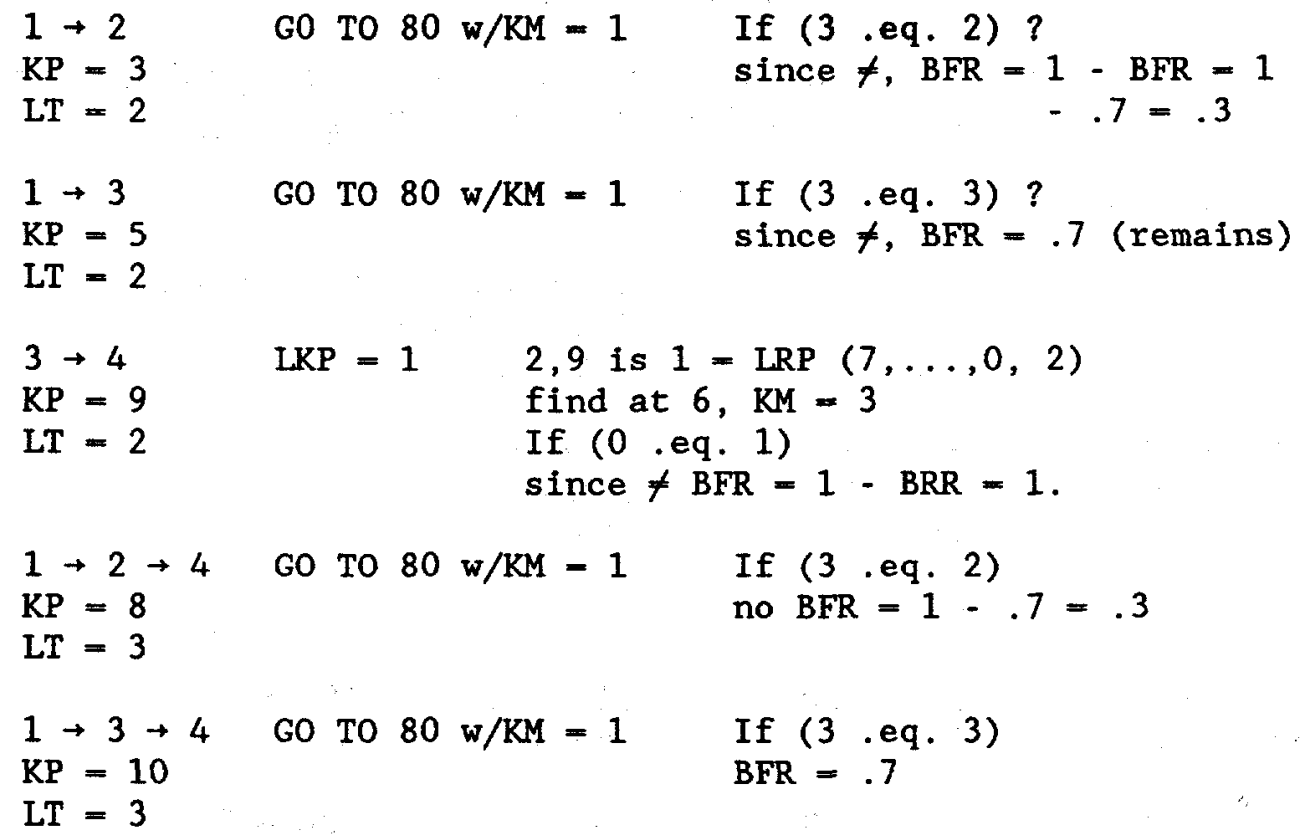

Subroutine BRANCH. In either case presented above, it may not be necessary to transport all subchains.

Example: $1 \rightarrow 2 \rightarrow 3 \rightarrow 4$

$$
\begin{aligned}
& \mathrm{T}^{1 / 2}(1)=10 \mathrm{y} \quad \mathrm{T}^{1 / 2}(2)=50 \mathrm{y} \quad \mathrm{T}^{1 / 2}(3)=10^{4} \mathrm{y} \\
& \mathrm{T}^{1 / 2}(4)=100 \mathrm{y}
\end{aligned}
$$

The production of iso \#4 by isos \#1 and \#2 might be insignificant compared to the production by \#3 over a time step. 
So, it is not necessary to transport subchains

$$
1 \rightarrow 2 \rightarrow 3 \rightarrow 4 \text { nor } 2 \rightarrow 3 \rightarrow 4
$$

In addition to setting up LPS, LPE, LRP, and DP, BRANCH decides necessary subchains. User can force all subchains by setting EPS - 0 in argument list (code change). Branch is called by SOURCE (update inventory to TRLSE), DTUPDT (age inventory by $\Delta t$-source), SETUP (age inventory by $\Delta t$ transport), with EPS $-10^{-8}, 10^{-5}, 10^{-4}$.

Branch looks at all paths from daughter iso IR to the end(s) of the chain. Can be up to 4 paths.

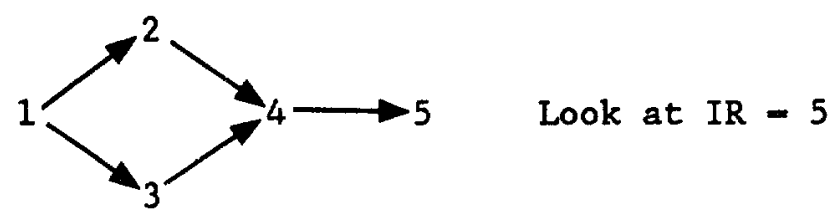

Branch finds 2 paths $1 \rightarrow 2 \rightarrow 4 \rightarrow 5$

$1 \rightarrow 3 \rightarrow 4 \rightarrow 5$

and will store in array IW.

The coding that is implemented for this example is as follows:

$$
\begin{array}{lll}
I R=5 & N B=0 & \text { (\# brand pts detected) } \\
& N P=1 \\
& K R=I R=5 \\
I=1 & \text { (\# paths) } \\
& I W(1,1)=5
\end{array}
$$

10 Loop

$$
\begin{array}{llll}
\mathrm{I}=2 & \mathrm{IW}(1,2)=4 & \text { no 2nd parent } & \mathrm{KR}=4 \\
\mathrm{I}=3 & \mathrm{IW}(1,3)=2 & \text { yes 2nd parent } & \mathrm{IPAR}(4,1)=-2 \\
& & \mathrm{NB}=1 \\
& & \mathrm{KR}=2 \\
& & \mathrm{KR}=1
\end{array}
$$

$$
I=4 \quad \operatorname{IW}(1,4)=1 \quad \text { no } 2 \text { nd parent }
$$

hit end of chain, go to 30

40 Loop

$$
\begin{aligned}
& \text { Detect } \operatorname{IPAR}(4,1)=-2(<0) \\
& \text { Set } \operatorname{IPAR}(4,1)=2 \text { \& go to } 50
\end{aligned}
$$


$50 \mathrm{NP}=2$

60 Loop $K=1, I^{(4)}$

If $\left(\operatorname{IW}(1, K)=J^{(4)}\right)$ go to 70 true for $K=2$

$70 I=3$

$$
\begin{aligned}
& \operatorname{IW}(2,1)=\operatorname{IW}(1,1) \quad 5 \\
& \operatorname{IW}(2,2)=\operatorname{IW}(1,2) \\
& \operatorname{KR}=3 \quad \operatorname{IW}(2,3)=3 \quad \mathrm{NB}=0
\end{aligned}
$$

10 Loop $I=4 \quad \operatorname{IW}(2,4)=1$ no 2 nd parent $K R=1$ hit end of chain, go to 30

$30 \mathrm{NB}=0$ so go to 100

At this point:

$$
\begin{aligned}
& \operatorname{IW}(1,1)=5 \\
& \operatorname{IW}(1,2)=4 \\
& \operatorname{IW}(1,3)=2 \\
& \operatorname{IW}(1,4)=1
\end{aligned}
$$

$\operatorname{IW}(2,1)=5$

$\operatorname{IW}(2,2)=4$

$\operatorname{IW}(2,3)=3$

$\operatorname{IW}(2,4)=1$

At $100 \rightarrow 200$

$$
\text { IS }=2 \quad \text { ISD }=2
$$

$\operatorname{LPS}(5)=\operatorname{LPE}(4)+1$

$L T=\operatorname{LPS}(5)$

$\operatorname{LRP}(\mathrm{LT}, 1)=5$

$\operatorname{LRP}(\mathrm{LT}, 2)=1$

$D P=e^{-\lambda \Delta t}$

$D(2)=\lambda_{4}$ define branch frac $=1$.

$L T=L T+1$ (stacking in LRP, DP)

$\mathrm{DP}(\mathrm{LT})=\operatorname{prod} 1 \rightarrow 2$ over $\Delta t$. 
APPENDIX E

Output Files for Sample Problems

E-1 
NEFTRAN II - PC VERSION RELEASED OCTOBER 1990

NEFTRAN II: SAMPLE PROBLEM \#1 FOR USER'S MANUAL (PC VERSION) EXECUTION DATE 11/12/1990 AND TIME 12: 7:40

PARAMETERS

GROUP1 - OPTIONS

GROUP - OPTIONS

STANDARD PRINT, NONZERO $\Rightarrow$ PRINT

OPTION VALUE OUTPUT-DESCRIPTION

1 LIST OF ALL PARAMETERS

1 THE NETWORK LEG PROPERTIES ARRAY

1 THE NETWORK JUNCTION PROPERTIES ARRAY

1 THE MIGRATION PATH PROPERTIES ARRAY

1 THE DECAY CHAIN PROPERTIES ARRAY

1 THE ELEMENT PROPERTIES ARRAY

1 FLUID VELOCITIES \& SOURCE/DISCH FLOWRATES

0 SUBCHAINS- DECAY, PRODUCTION, \& VELOCITIES

0 SPACE \& TIME STEPS AND SOURCE TYPE

TIME DEPENDENT OUTPUT

- IF + N, DISCHARGE RATES EVERY NTH TIME STEP

- IF -N, CONCENTRATIONS EVERY NTH TIME STEP

111 DISCHARGE SUMMARY

EXTERNAL FILES, NONZERO $\Rightarrow$ FILE WRITTEN/READ

OPTION VALUE CORRESPONDING FILE

120 WRITE SRATE.DAT, SOURCE RATES $(\mathrm{C} i / \mathrm{Y})$

131 WRITE DRATE.DAT, DISCHARGE RATES $(\mathrm{Ci} / \mathrm{Y})$

14 O NOT CURRENTLY USED

15 O READ SAMP.DAT, SAMPLED DATA NORMALLY FOR REPEATED TRIALS

16 READ VFIELD.DAT, TIME-DEPENDENT VELOCITIES

17 READ SFLOW.DAT, TIME-DEPENDENT FLOWRATES

RUN CONTROLS

OPTION VALUE DESCRIPTION

$18 \quad 1$ USE DVM(O) OR ANALYTIC SOLN(1)

1900 SOLVE NETWORK(0) OR INPUT VELOCITIES $(1)$

200 LEACH(0), SOLUBILITIES(1), OR BOTH(2)

210 FLOWTHRU $(0), \operatorname{MIXCELLL(1),OR~CHOOSE(2)}$

220 CONSTANT(0) OR EXPONENTIAL(1) LEACH RATE

230 FORCE(1) USE OF LEG-TO-LEG TRANSFER ALGORITHM

DEBUG PRINTS, NONZERO $\Rightarrow$ PRINT

OPTION VALUE OUTPUT-DESCRIPTION

$24 \quad 0$ DVM MULTIPLIERS FOR TRANSPORT, DISCHARGE, AND SOURCE

250 DIAGNOSTIC INFO FOR SUBROUTINE BAND 


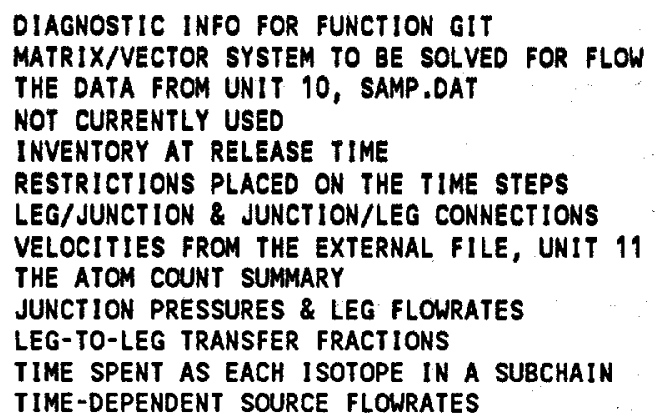

GROUP2 - PROBLEM SIZES

VALUE DESCRIPTION

NUMBER OF NETWORK LEGS

NUMBER OF NETWORK JUNCTIONS

NUMBER OF MIGRATION PATH LEGS

NUMBER OF DECAY CHAINS INPUT

NUMBER OF ELEMENTS INPUT

$$
\begin{array}{lc}
\text { DECAY CHAINS } \\
\text { NUMBER } & \text { INCLUSION } \\
\text { MEMBERS } & \text { INDEX } \\
3 & 0
\end{array}
$$

GROUP3 - SOURCE/FLOW PARAMETERS

VALUE

$1.0000 E+00$

$0.0000 E+00$

$0.0000 E+00$

$1.0000 E-05$

$0.0000 E+00$

$0.0000 E+00$

DESCR IPTION

FRACTION OF SOURCE ACCESSED

PORE VOLUME CONTAINING SOURCE ( $f * * 3)$

PORE-AREA FOR SOURCE REGIME $(f t * * 2)$

LEACH RATE $(1 / y)$

DENSITY OF SOURCE FLUID (lb/ft**3)

PORE-AREA AT DISCHARGE $(\mathrm{ft} * \star 2)$

VALUE

TIME TO END OF SIMULATION (Y)

$0.0000 E+00$ TIME OF ONSET OF MIGRATION $(y)$

$0.0000 E+00$ TIME OF ONSET OF LEACHING $(y)$

$0.0000 E+00$ TIME STEP FOR SOURCE $(y)$

TRANSPORT TIME STEPS FOR EACH CHAIN CHAIN\# TIME STEP(Y)

$1 \quad 0.000 E+00$

GROUP5 - INCREMENT DETERMINATION

DEFAULT values utilized

Network Properties Array 


\begin{tabular}{|c|c|c|c|c|c|c|}
\hline $\begin{array}{l}\text { eg } \\
\# \\
1 \\
2 \\
3 \\
4 \\
5 \\
6 \\
7 \\
8 \\
9 \\
10\end{array}$ & $\begin{array}{c}\text { Inlet } \\
\text { jct } \\
1 \\
4 \\
5 \\
10 \\
2 \\
9 \\
11 \\
12 \\
6 \\
8 \\
9 \\
11 \\
6 \\
7 \\
12 \\
13\end{array}$ & $\begin{array}{c}\text { Outlet } \\
\text { jct } \\
4 \\
5 \\
10 \\
3 \\
9 \\
11 \\
12 \\
13 \\
4 \\
5 \\
6 \\
8 \\
7 \\
8 \\
10 \\
3\end{array}$ & $\begin{array}{c}\text { Length } \\
\text { (ft) } \\
1.450 \mathrm{ft}+04 \\
8.000 \mathrm{0}+03 \\
3.800 \mathrm{0}+04 \\
1.000 \mathrm{0}+05 \\
1.450 \mathrm{0}+04 \\
8.000 \mathrm{0}+03 \\
3.800 \mathrm{0}+04 \\
1.000 \mathrm{0}+05 \\
6.000 \mathrm{0}+02 \\
4.965 \mathrm{E}+02 \\
5.000 \mathrm{E}+02 \\
6.035 \mathrm{E}+02 \\
4.000 \mathrm{E}+03 \\
4.000 \mathrm{0}+03 \\
1.100 \mathrm{0}+03\end{array}$ & $\begin{array}{r}\text { Area } \\
(\mathrm{sq}-\mathrm{ft}) \\
6.000 \mathrm{E}+06\end{array}$ & $\begin{array}{l}\text { Hydraul ic } \\
K \quad(f t / d) \\
5.000 E+01 \\
5.000 E+01 \\
5.000 E+01 \\
5.000 E+01 \\
4.000 E+01 \\
4.000 E+01 \\
4.000 E+01 \\
4.000 E+01 \\
1.000 E-01 \\
1.000 E+01 \\
1.670 E-06 \\
1.500 E-06 \\
1.000 E+01 \\
1.000 E+01 \\
1.570 E-06 \\
2.500 E+00\end{array}$ & $\begin{array}{c}\text { Brine } \\
\text { Conc. } \\
0.000 E+00 \\
0.000 E+00 \\
0.000 E+00 \\
0.000 E+00 \\
0.000 E+00 \\
0.000 E+00 \\
0.000 E+00 \\
0.000 E+00 \\
6.700 E-01 \\
1.000 E+00 \\
1.000 E+00 \\
1.000 E+00 \\
1.000 E+00 \\
1.000 E+00 \\
1.000 E+00 \\
0.000 E+00\end{array}$ \\
\hline
\end{tabular}

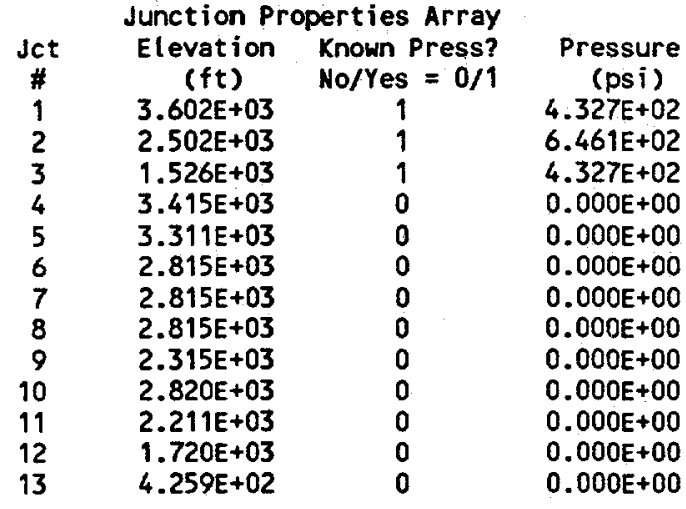

$\begin{array}{cccccccc}\text { Leg } & \text { Dispersivity } & \text { Migration Path Properties Array } \\ \text { \# } & \text { Space Step } & \text { Diffusion? } & \text { Mobile } & \text { Immobile } & \text { Mass Xfer } & \text { Velocity } \\ 13 & 5.000 E+02 & 0.000 E+00 & 0 & 3.000 E-01 & 0.000 E+00 & 0.000 E+00 & 0.000 E+00 \\ 14 & 0.000 E+00 & 0.000 E+00 & 0 & 3.000 E-01 & 0.000 E+00 & 0.000 E+00 & 0.000 E+00 \\ 10 & 0.000 E+00 & 0.000 E+00 & 0 & 1.500 E-01 & 0.000 E+00 & 0.000 E+00 & 0.000 E+00 \\ 3 & 0.000 E+00 & 0.000 E+00 & 0 & 3.000 E-01 & 0.000 E+00 & 0.000 E+00 & 0.000 E+00 \\ 4 & 0.000 E+00 & 0.000 E+00 & 0 & 3.000 E-01 & 0.000 E+00 & 0.000 E+00 & 0.000 E+00\end{array}$

\begin{tabular}{|c|c|c|c|c|c|c|c|c|c|c|}
\hline \multicolumn{11}{|c|}{ Decay Chain Array } \\
\hline $\begin{array}{l}\text { Name } \\
\text { A6 } \\
\text { DECAY }\end{array}$ & $\begin{array}{l}\text { Atomic } \\
\text { Mass }\end{array}$ & $\begin{array}{l}\text { Elem. } \\
\text { Index }\end{array}$ & $\begin{array}{l}\text { Local } \\
\text { Index }\end{array}$ & $\begin{array}{c}\text { Parent } \\
\# 1\end{array}$ & $\begin{array}{c}\text { Parent } \\
\# 2\end{array}$ & $\begin{array}{l}\text { Fraction } \\
\text { From } \# 1\end{array}$ & $\begin{array}{l}\text { Fraction } \\
\text { From } \# 2\end{array}$ & $\begin{array}{l}\text { Inventory } \\
\text { (Ci) }\end{array}$ & $\begin{array}{c}\text { Half-Life } \\
(y)\end{array}$ & $\begin{array}{c}\text { Weighting } \\
\text { Factor }\end{array}$ \\
\hline $\begin{array}{l}\text { NP237 } \\
\text { U233 } \\
\text { TH229 }\end{array}$ & $\begin{array}{l}237.0 \\
233.0 \\
229.0\end{array}$ & $\begin{array}{l}1 \\
2 \\
3\end{array}$ & $\begin{array}{l}1 \\
2 \\
3\end{array}$ & $\begin{array}{l}0 \\
1 \\
2\end{array}$ & $\begin{array}{l}0 \\
0 \\
0\end{array}$ & $\begin{array}{l}0.000 E+00 \\
1.000 E+00 \\
1.000 E+00\end{array}$ & $\begin{array}{l}0.000 E+00 \\
0.000 E+00 \\
0.000 E+00\end{array}$ & $\begin{array}{l}1.000 E+03 \\
1.000 E+03 \\
1.000 E+03\end{array}$ & $\begin{array}{l}2.140 E+06 \\
1.620 E+05 \\
7.300 E+03\end{array}$ & $\begin{array}{l}1.000 E+00 \\
1.000 E+00 \\
1.000 E+00\end{array}$ \\
\hline
\end{tabular}


Element Properties Array

Elem. Solubility Leg Mobil Immobile

Index ( $/ \mathrm{g})$ \# Rd Rd

$1 \quad 0.000 E+00 \quad 13 \quad 1.000 E+00 \quad 0.000 E+00$

$1.000 E+00 \quad 0.000 E+00$

$1.000 E+00 \quad 0.000 E+00$

$6.357 E+02 \quad 0.000 E+00$

$6.357 E+02 \quad 0.000 E+00$

2

$0.000 E+00$

$1.000 E+00$

$1.000 E+00 \quad 0.000 E+00$

$6.357 E+02 \quad 0.000 E+00$

$6.357 E+02 \quad 0.000 E+00$

3

$\begin{array}{rrrr}0.000 E+00 & 13 & 1.000 E+00 & 0.000 E+00 \\ & 14 & 1.000 E+00 & 0.000 E+00 \\ 10 & 1.000 E+00 & 0.000 E+00 \\ 3 & 6.357 E+02 & 0.000 E+00 \\ & 4 & 6.357 E+02 & 0.000 E+00\end{array}$

\begin{tabular}{rrr} 
THE LJ & ARRAY \\
LEG & \multicolumn{2}{c}{ JCTS } \\
1 & 1 & 4 \\
2 & 4 & 5 \\
3 & 5 & 10 \\
4 & 10 & 3 \\
5 & 2 & 9 \\
6 & 9 & 11 \\
7 & 11 & 12 \\
8 & 12 & 13 \\
9 & 6 & 4 \\
10 & 8 & 5 \\
11 & 9 & 6 \\
12 & 11 & 8 \\
13 & 6 & 7 \\
14 & 7 & 8 \\
15 & 12 & 10 \\
16 & 13 & 3
\end{tabular}

THE JL ARRAY

$\begin{array}{rrrr}\text { JCT } & & \text { LEGS } \\ 1 & 1 & 0 & 0 \\ 2 & 5 & 0 & 0 \\ 3 & -4 & -16 & 0 \\ 4 & -1 & -9 & 2 \\ 5 & -2 & -10 & 3 \\ 6 & -11 & 9 & 13 \\ 7 & -13 & 14 & 0 \\ 8 & -12 & -14 & 10 \\ 9 & -5 & 6 & 11 \\ 10 & -3 & -15 & 4\end{array}$


PRESSURES AT THE LEG JUNCTIONS

JUNCTION

FLUID PRESSURE (psi)

1
2
3
4
5
6
7
8
9
10
11
12
13

$4.3270 E+02$

$6.4610 E+02$

4.3270E+02

$4.3270 E+02$

$4.3270 E+02$

$7.2025 E+02$

$7.1978 E+02$

$7.1931 E+02$

$6.6992 E+02$

$4.3270 E+02$

$6.8307 E+02$

$7.4550 E+02$

$9.0980 E+02$

VOLUMETRIC FLOW RATE FOR EACH LEG LEG FLOW $\mathrm{ft} * \star 3 / \mathrm{Y}$

$\begin{array}{rr}1 & 1.4177 \mathrm{E}+09 \\ 2 & 1.41777 \mathrm{E}+09 \\ 3 & 1.4177 \mathrm{E}+09 \\ 4 & 1.4177 \mathrm{E}+09 \\ 5 & 2.4040 \mathrm{E}+08 \\ 6 & 2.4040 \mathrm{E}+08 \\ 7 & 2.4040 \mathrm{E}+08 \\ 8 & 2.4040 \mathrm{E}+08 \\ 9 & -3.7375 \mathrm{E}+02 \\ 10 & 3.7375 \mathrm{E}+02 \\ 11 & -6.0667 \mathrm{E}-04 \\ 12 & -5.0893 \mathrm{E}-04 \\ 13 & 3.7375 \mathrm{E}+02 \\ 14 & 3.7375 \mathrm{E}+02 \\ 15 & -2.1310 \mathrm{E}-04 \\ 16 & 2.4040 \mathrm{E}+08\end{array}$

FOR STEADY-STATE FLOW

LEG NO. PORE VELOCITY

$\mathrm{ft} / \mathrm{Y}$

$\begin{array}{rc} & f t / y \\ 13 & 2.3071 E+00 \\ 14 & 2.3071 E+00 \\ 10 & 2.4917 E+03 \\ 3 & 7.8759 E+02 \\ 4 & 7.8759 E+02\end{array}$

PATH LENGTH FROM SOURCE BOUNDARY $(f t)=1.4250 E+05$ AVERAGE FLUID VELOCITY $(\mathrm{ft} / \mathrm{y})=7.4637 \mathrm{E}+01$ 
FLOW RATE THROUGH SOURCE $=3.7375 E+02 \mathrm{ft} * * 3 / \mathrm{Y}$

FLOW RATE AT DISCHARGE $=1.4177 \mathrm{E}+09 \mathrm{ft} * 3 / \mathrm{Y}$

RADIONUCLIDE DISCHARGE RATE (CI/Y)

$$
\text { YEAR NP237 U233 }
$$

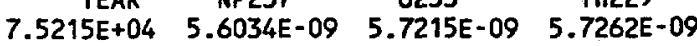

$7.6973 \mathrm{E}+04 \quad 2.2369 \mathrm{E}-08 \quad 2.2850 \mathrm{E}-08 \quad 2.2869 \mathrm{E}-08$

$7.8731 E+04$ 8.0186E-08 $8.1943 \mathrm{E}-08$ 8.2013E-08

$\begin{array}{llll}8.0489 E+04 & 2.6000 E-07 & 2.6580 E-07 & 2.6604 E-07\end{array}$

$\begin{array}{llll}8.2247 E+04 & 7.6767 E-07 & 7.8513 E-07 & 7.8583 E-07\end{array}$

$8.4006 E+04 \quad 2.0768 E-06 \quad 2.1249 E-06 \quad 2.1268 E-06$

$8.5764 E+04 \quad 5.1772 E-06 \quad 5.2991 E-06 \quad 5.3040 E-06$

$8.7522 \mathrm{E}+04 \quad 1.1955 \mathrm{E}-05$ 1.2242E-05 $1.2254 \mathrm{E}-05$

$8.9280 E+04 \quad 2.5700 E-05 \quad 2.6326 E-05 \quad 2.6352 E-05$

$9.1038 E+04 \quad 5.1663 E-05 \quad 5.2943 E-05 \quad 5.2995 E-05$

$9.2796 E+04 \quad 9.7534 E-05 \quad 9.9988 E-05 \quad 1.0009 E-04$

$9.4554 E+04 \quad 1.7362 E-04 \quad 1.7805 E-04 \quad 1.7823 E-04$

$9.6312 E+04 \quad 2.9250 E-04 \quad 3.0008 E-04 \quad 3.0039 E-04$

$9.8070 E+04 \quad 4.6804 E-04 \quad 4.8037 E-04 \quad 4.8087 E-04$

$9.9828 \mathrm{E}+04 \quad 7.1378 \mathrm{E}-04 \quad 7.3285 \mathrm{E}-04 \quad 7.3363 \mathrm{E}-04$

$1.0159 E+05 \quad 1.0407 E-03 \quad 1.0689 E-03 \quad 1.0701 E-03$

$\begin{array}{llll}1.0334 E+05 & 1.4553 E-03 & 1.4953 E-03 & 1.4970 E-03\end{array}$

$\begin{array}{llll}1.0510 E+05 & 1.9576 E-03 & 2.0121 E-03 & 2.0144 E-03\end{array}$

$\begin{array}{llll}1.0686 E+05 & 2.5401 E-03 & 2.6119 E-03 & 2.6148 E-03\end{array}$

$\begin{array}{llll}1.0862 E+05 & 3.1885 E-03 & 3.2797 E-03 & 3.2835 E-03\end{array}$

$\begin{array}{llll}1.1038 E+05 & 3.8821 E-03 & 3.9946 E-03 & 3.9993 E-03\end{array}$

$1.1214 \mathrm{E}+05 \quad 4.5970 \mathrm{E}-03$
$1.1389 \mathrm{E}+05$

$1.1389 E+05 \quad 5.3077 E-03 \quad 5.4653 E-03 \quad 5.4719 E-03$

$\begin{array}{llll}1.1565 E+05 & 5.9905 E-03 & 6.1706 E-03 & 6.1781 E-03\end{array}$

$\begin{array}{lllll}1.1741 E+05 & 6.6255 E-03 & 6.8270 E-03 & 6.8355 E-03\end{array}$

$1.1917 \mathrm{E}+05 \quad 7.1976 \mathrm{E}-03 \quad 7.4192 \mathrm{E}-03 \quad 7.4285 \mathrm{E}-03$

$1.2093 \mathrm{E}+05 \quad 7.6980 \mathrm{E}-03 \quad 7.9376 \mathrm{E}-03 \quad 7.9477 \mathrm{E}-03$

$\begin{array}{llll}1.2268 E+05 & 8.1230 E-03 & 8.3787 E-03 & 8.3895 E-03\end{array}$

$\begin{array}{llll}1.2444 E+05 & 8.4741 E-03 & 8.7438 E-03 & 8.7552 E-03\end{array}$

$1.2620 E+05 \quad 8.7564 E-03 \quad 9.0381 E-03 \quad 9.0500 E-03$

$\begin{array}{llll}1.2796 E+05 & 8.9774 E-03 & 9.2693 E-03 & 9.2817 E-03\end{array}$

$1.2972 \mathrm{E}+05 \quad 9.1459 \mathrm{E}-03 \quad 9.4465 \mathrm{E}-03 \quad 9.4592 \mathrm{E}-03$

$1.3147 E+05 \quad 9.2711 E-03 \quad 9.5790 E-03 \quad 9.5920 E-03$

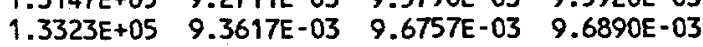

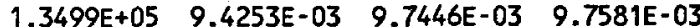

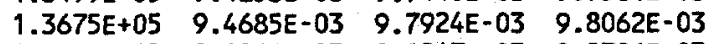

$\begin{array}{llll}1.3851 E+05 & 9.4966 E-03 & 9.8247 E-03 & 9.8386 E-03\end{array}$

$\begin{array}{llllll}1.4026 E+05 & 9.5140 E-03 & 9.8457 E-03 & 9.8599 E-03\end{array}$

$\begin{array}{llll}1.4202 E+05 & 9.5237 E-03 & 9.8589 E-03 & 9.8732 E-03\end{array}$

$\begin{array}{lllll}1.4378 E+05 & 9.5282 E-03 & 9.8667 E-03 & 9.8811 E-03\end{array}$

$1.4554 \mathrm{E}+05 \quad 9.5292 \mathrm{E}-03 \quad 9.8707 \mathrm{E}-03 \quad 9.8853 \mathrm{E}-03$
03

$\begin{array}{llll}1.4730 E+05 & 9.5278 E-03 & 9.8723 E-03 & 9.8870 E-03\end{array}$

$1.4906 \mathrm{E}+05 \quad 9.5249 \mathrm{E}-03 \quad 9.8723 \mathrm{E}-03 \quad 9.8872 \mathrm{E}-03$

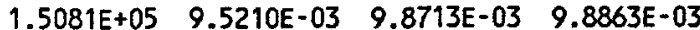

$\begin{array}{llll}1.5257 E+05 & 9.5165 E-03 & 9.8696 E-03 & 9.8847 E-03\end{array}$

$1.5433 E+05 \quad 9.5116 E-03 \quad 9.8675 E-03 \quad 9.8828 E-03$
0.5603

$1.5609 E+05 \quad 9.5066 E-03 \quad 9.8652 E-03 \quad 9.8806 E-03$

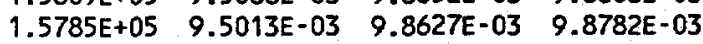

$\begin{array}{llll}1.5960 E+05 & 9.4960 E-03 & 9.8601 E-03 & 9.8757 E-03\end{array}$

$\begin{array}{llll}1.6136 E+05 & 9.4907 E-03 & 9.8574 E-03 & 9.8731 E-03\end{array}$ 
$1.6312 E+05$

$1.6488 \mathrm{E}+05$

$1.6664 E+05$

$1.6839 E+05$

$1.7015 E+05$

$1.7191 \mathrm{E}+05$

1. $7367 E+05$

$1.7543 \mathrm{E}+05$

$1.7719 E+05$

$1.7894 E+05$

$1.8070 E+05$

$1.8246 E+05$

$1.8422 E+05$

$1.8598 \mathrm{E}+05$

$1.8773 E+05$

$1.8949 \mathrm{E}+05$

$1.9125 E+05$

$1.9301 E+05$

$1.9477 \mathrm{E}+05$

$1.9652 E+05$

$1.9828 E+05$

$2.0004 E+05$

$2.0180 E+05$

$2.0356 E+05$

$2.0531 E+05$

$2.0707 \mathrm{E}+05$

2. $0883 \mathrm{E}+05$

2. $1059 E+05$

2.1235E+05

2. $1411 E+05$

$2.1586 \mathrm{E}+05$

$2.1762 E+05$

2. $1938 \mathrm{E}+05$

$2.2114 \mathrm{E}+05$

$2.2290 E+05$

2. $2465 E+05$

2. $2641 E+05$

$2.2817 E+05$

2.2993E+05

2.3169E+05

$2.3344 \mathrm{E}+05$

$2.3520 E+05$

2.3696E+05

$2.3872 E+05$

$2.4048 E+05$

$2.4224 E+05$

$2.4399 E+05$

$2.4575 E+05$

$2.4751 E+05$

$2.4927 \mathrm{E}+05$

$2.5103 \mathrm{E}+05$

$2.5103 E+05$

$1.0000 E+06$
9.4853E-03 9.8546E-03 $9.8705 \mathrm{E}-03$

9.4799E-03 9.8519E-03 9.8679E-03

9.4746E-03 9.8491E-03 9.8652E-03

$\begin{array}{lll}9.4692 E-03 & 9.8463 E-03 & 9.8625 E-03\end{array}$

\begin{tabular}{l}
$9.4638 E-03 \quad 9.8434 E-03 \quad 9.8598 E-03$ \\
\hline
\end{tabular}

9.4584E-03 $9.8405 E-03 \quad 9.8571 E-03$

9.4530E-03 9.8377E-03 9.8543E-03

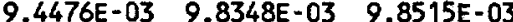

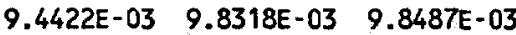

9.4368E-03 9.8288E-03 9.8458E-03

$9.4312 E-03$
$9.8256 E-03 \quad 9.8427 E-03$

\begin{tabular}{l}
$9.4253 E-03 \quad 9.8221 E-03 \quad 9.8393 E-03$ \\
\hline
\end{tabular}

9.4185E-03 9.8176E-03 9.8349E-03

9.4098E-03 9.8112E-03 9.8286E-03

$9.3973 E-03 \quad 9.8006 E-03 \quad 9.8182 E-03$

9.3775E-03 $9.7825 E-03 \quad 9.8001 E-03$

9.3452E-03 9.7513E-03 9.7689E-03

$9.2925 E-03 \quad 9.6988 E-03 \quad 9.7164 E-03$

9.2091E-03 9.6142E-03 9.6319E-03

$9.0828 E-03 \quad 9.4847 E-03 \quad 9.5022 E-03$

8.9000E-03 9.2961E-03 9.3134E-03

$8.6477 \mathrm{E}-03 \quad 9.0349 \mathrm{E}-03 \quad 9.0518 \mathrm{E}-03$

8.3158E-03 8.6903E-03 8.7066E-03

$\begin{array}{lll}7.8987 E-03 & 8.2564 E-03 & 8.2720 E-03\end{array}$

7.3973E-03 7.7341E-03 7.7488E-03

6.8194E-03 7.1317E-03 $\quad 7.1453 \mathrm{E}-03$

$6.1800 E-03 \quad 6.4645 E-03 \quad 6.4769 E-03$

5.4993E-03 5.7539E-03 5.7650E-03

4.8012E-03 5.0246E-03 5.0343E-03

4.1099E-03 4.3021E-03 4.3105E-03

$3.4481 E-03 \quad 3.6103 E-03 \quad 3.6174 E-03$

$\begin{array}{lll}2.8348 E-03 & 2.9688 E-03 & 2.9746 E-03\end{array}$

2.2836E-03 2.3921E-03 2.3968E-03

$\begin{array}{lll}1.8026 E-03 & 1.8887 E-03 \quad 1.8924 E-03\end{array}$

$\begin{array}{lll}1.3946 E-03 & 1.4615 E-03 & 1.4645 E-03\end{array}$

$1.0578 E-03 \quad 1.1088 E-03 \quad 1.1110 E-03$

7.8681E-04 8.2493E-04 8.2660E-04

5.7415E-04 6.0210E-04 6.0333E-04

4.1120E-04 4.3131E-04 4.3220E-04

$2.8916 E-04 \quad 3.0337 E-04 \quad 3.0400 E-04$

$\begin{array}{lll}1.9975 E-04 & 2.0961 E-04 & 2.1005 E-04\end{array}$

$1.3561 E-04 \quad 1.4234 E-04 \quad 1.4263 E-04$

$9.0527 E-05 \quad 9.5037 E-05 \quad 9.5235 E-05$

$\begin{array}{lll}5.9446 E-05 & 6.2420 E-05 & 6.2551 E-05\end{array}$

$3.8419 E-05 \quad 4.0350 E-05 \quad 4.0434 E-05$

2.4448E-05 2.5682E-05 2.5736E-05

$1.5325 \mathrm{E}-05 \quad 1.6102 \mathrm{E}-05 \quad 1.6136 \mathrm{E}-05$

9.4676E-06 9.9496E-06 9.9708E-06

$\begin{array}{lll}5.7664 E-06 & 6.0613 E-06 & 6.0742 E-06\end{array}$

3.4649E-06 3.6428E-06 3.6507E-06

$\begin{array}{lll}2.0540 E-06 & 2.1599 E-06 & 2.1646 E-06\end{array}$

$0.0000 E+00 \quad 0.0000 E+00 \quad 0.0000 E+00$

$0.0000 E+00 \quad 0.0000 E+00 \quad 0.0000 E+00$ 
ISOTOPE DISCHARGE SUMMARY FOR ALL 3 ISOTOPES

PEAK-RATE PEAK-TIME TOTAL(Ci) TOTAL(WEIGHTED)

CHAIN 1

$\begin{array}{lllll}\text { NP237 } & 9.5292 E-03 & 1.4554 E+05 & 9.4859 E+02 & 9.4859 E+02 \\ \text { U233 } & 9.8723 E-03 & 1.4906 E+05 & 9.8517 E+02 & 9.8517 E+02\end{array}$

$\begin{array}{lllll}\text { TH229 } & 9.8872 E-03 & 1.4906 E+05 & 9.8674 E+02 & 9.8674 E+02\end{array}$

TOTAL(WEIGHTED) $=2.9205 E+03$

ABOUT TO READ DATA SET \# 2

THE INPUT ITEMS TO BE RE-READ ARE. ALL OPTIONS

PARAMETERS

GROUP1 - OPTIONS

-

STANDARD PRINT, NONZERO $\Rightarrow>$ PRINT

OPTION VALUE OUTPUT-DESCRIPTION

11 LIST OF ALL PARAMETERS

2. 0 THE NETWORK LEG PROPERTIES ARRAY

3 T 0 THE NETWORK JUNCTION PROPERTIES ARRAY

4.0 THE MIGRATION PATH PROPERTIES ARRAY

5 THE DECAY CHAIN PROPERTIES ARRAY

60 THE ELEMENT PROPERTIES ARRAY

71 FLUID VELOCITIES \& SOURCE/DISCH FLOWRATES

81 SUBCHAINS- DECAY, PRODUCTION, \& VELOCITIES

$9 \quad 1$ SPACE \& TIME STEPS AND SOURCE TYPE

101 TIME DEPENDENT OUTPUT

- IF + N, DISCHARGE RATES EVERY NTH TIME STEP

111 DISCHARGE SUMMARY

EXTERNAL FILES, NONZERO $\Rightarrow$ FILE WRITTEN/READ

OPTION VALUE CORRESPONDING FILE

12 O WRITE SRATE.DAT, SOURCE RATES ( $\mathrm{C} i / \mathrm{Y}$ )

131 WRITE DRATE.DAT, DISCHARGE RATES $(\mathrm{C} i / \mathrm{Y})$

140 NOT CURRENTLY USED

15 O READ SAMP.DAT, SAMPLED DATA NORMALLY FOR REPEATED TRIALS

160 READ VFIELD.DAT, TIME-DEPENDENT VELOCITIES

17 READ SFLOW.DAT, TIME-DEPENDENT FLOWRATES

RUN CONTROLS

OPTION VALUE DESCRIPTION

180 USE DVM(0) OR ANALYTIC SOLN(1)

1900 SOLVE NETWORK(0) OR INPUT VELOCITIES(1)

20 O LEACH(0), SOLUBILITIES(1), OR BOTH(2)

21 O FLOWTHRU(0), MIXCELL(1), OR CHOOSE(2)

220 CONSTANT (0) OR EXPONENTIAL(1) LEACH RATE

230 FORCE(1) USE OF LEG-TO-LEG TRANSFER ALGORITHM 
DEBUG PRINTS, NONZERO $=>$ PRINT

OPTION VALUE OUTPUT-DESCRIPTION

2400 DVM MULTIPLIERS FOR TRANSPORT, DISCHARGE, AND SOURCE

250 DIAGNOSTIC INFO FOR SUBROUTINE BAND

2600 DIAGNOSTIC INFO FOR FUNCTION GIT

27 O MATRIX/VECTOR SYSTEM TO BE SOLVED FOR FLOW

28.0 THE DATA FROM UNIT 10, SAMP.DAT

290 NOT CURRENTLY USED

30 INVENTORY AT RELEASE TIME

31 O RESTRICTIONS PLACED ON THE TIME STEPS

320 LEG/JUNCTION \& JUNCTION/LEG CONNECTIONS

3300 VELOCITIES FROM THE EXTERNAL FILE, UNIT 11

340 THE ATOM COUNT SUMMARY

350 JUNCTION PRESSURES \& LEG FLOWRATES

3600 LEG-TO-LEG TRANSFER FRACTIONS

37 TIME SPENT AS EACH ISOTOPE IN A SUBCHAIN

380 TIME-DEPENDENT SOURCE FLOWRATES

***WARNING*** "DENSITY OF SOURCE FLUID" NOT USED

***WARNING*** PORE VELOCITIES NOT USED, SINCE THEY ARE OBTAINED FROM NETWORK OR EXTERNAL FILE

\begin{tabular}{cc} 
FOR STEADY-STATE FLOW \\
\hline LEG NO. & PORE VELOCITY \\
& ft/Y \\
13 & $2.3071 E+00$ \\
14 & $2.3071 E+00$ \\
10 & $2.4917 E+03$ \\
3 & $7.8759 E+02$ \\
4 & $7.8759 E+02$
\end{tabular}

PATH LENGTH FROM SOURCE BOUNDARY $(\mathrm{ft})=1.4250 E+05$ AVERAGE FLUID VELOCITY $(\mathrm{ft} / \mathrm{y})=7.4637 \mathrm{E}+01$

FLOW RATE THROUGH SOURCE $=3.7375 E+02 \mathrm{ft} * * 3 / \mathrm{Y}$ FLOW RATE AT DISCHARGE $=1.4177 \mathrm{E}+09 \mathrm{ft} * * 3 / \mathrm{Y}$ DECAY/PRODUCTION FACTORS AND VELOCITIES FOR EACH SUBCHAIN TRANSPORTED
SUBCHAIN \#

1
2
3
4
5

\begin{tabular}{|c|c|c|}
\hline (DECAY) & NP237 & $9.93539 E-01$ \\
\hline $\begin{array}{l}\text { (DECAY) } \\
\text { NP237 }\end{array}$ & $\begin{array}{r}U 233 \\
>U 233\end{array}$ & $\begin{array}{l}9.17939 E-01 \\
6.19170 E-03\end{array}$ \\
\hline $\begin{array}{l}\text { (DECAY) } \\
\text { U233 }\end{array}$ & $\begin{array}{l}\text { TH229 } \\
\text { > TH229 }\end{array}$ & $\begin{array}{l}1.49546 E-01 \\
3.62590 E-02\end{array}$ \\
\hline
\end{tabular}

VELOCITY INFORMATION FOR EACH SUBCHAIN SINGLE LEG OPTION

\# MIN VELOCITY MEAN VELOCITY

$1 \quad 8.92003 E-01 \quad 1.25969 E+00$

MAX. VELOCITY

MEAN COURANT NO.

$1.62738 E+00 \quad 8.84536 E+00$ 


$\begin{array}{llll}8.92003 E-01 & 1.25969 E+00 & 1.62738 E+00 & 8.84536 E+00 \\ 8.92003 E-01 & 1.25969 E+00 & 1.62738 E+00 & 8.84536 E+00 \\ 8.92003 E-01 & 1.25969 E+00 & 1.62738 E+00 & 8.84536 E+00 \\ 8.92003 E-01 & 1.25969 E+00 & 1.62738 E+00 & 8.84536 E+00 \\ 8.92003 E-01 & 1.25969 E+00 & 1.62738 E+00 & 8.84536 E+00\end{array}$

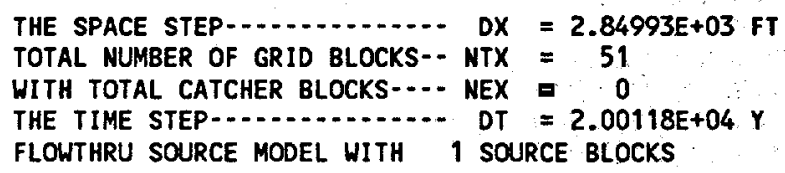




$\begin{array}{llll}7.6045 E+05 & 0.0000 E+00 & 0.0000 E+00 & 0.0000 E+00 \\ 7.8046 E+05 & 0.0000 E+00 & 0.0000 E+00 & 0.0000 E+00 \\ 8.0047 E+05 & 0.0000 E+00 & 0.0000 E+00 & 0.0000 E+00 \\ 8.2048 E+05 & 0.0000 E+00 & 0.0000 E+00 & 0.0000 E+00 \\ 8.4049 E+05 & 0.0000 E+00 & 0.0000 E+00 & 0.0000 E+00 \\ 8.6051 E+05 & 0.0000 E+00 & 0.0000 E+00 & 0.0000 E+00 \\ 8.8052 E+05 & 0.0000 E+00 & 0.0000 E+00 & 0.0000 E+00 \\ 9.0053 E+05 & 0.0000 E+00 & 0.0000 E+00 & 0.0000 E+00 \\ 9.2054 E+05 & 0.0000 E+00 & 0.0000 E+00 & 0.0000 E+00 \\ 9.4055 E+05 & 0.0000 E+00 & 0.0000 E+00 & 0.0000 E+00 \\ 9.6056 E+05 & 0.0000 E+00 & 0.0000 E+00 & 0.0000 E+00 \\ 9.8058 E+05 & 0.0000 E+00 & 0.0000 E+00 & 0.0000 E+00 \\ 1.0006 E+06 & 0.0000 E+00 & 0.0000 E+00 & 0.0000 E+00 \\ 1.0206 E+06 & 0.0000 E+00 & 0.0000 E+00 & 0.0000 E+00\end{array}$

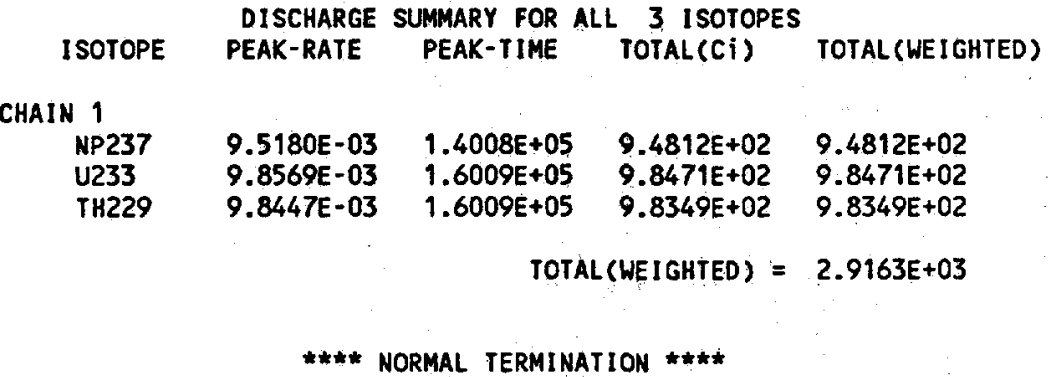


PARAMETERS

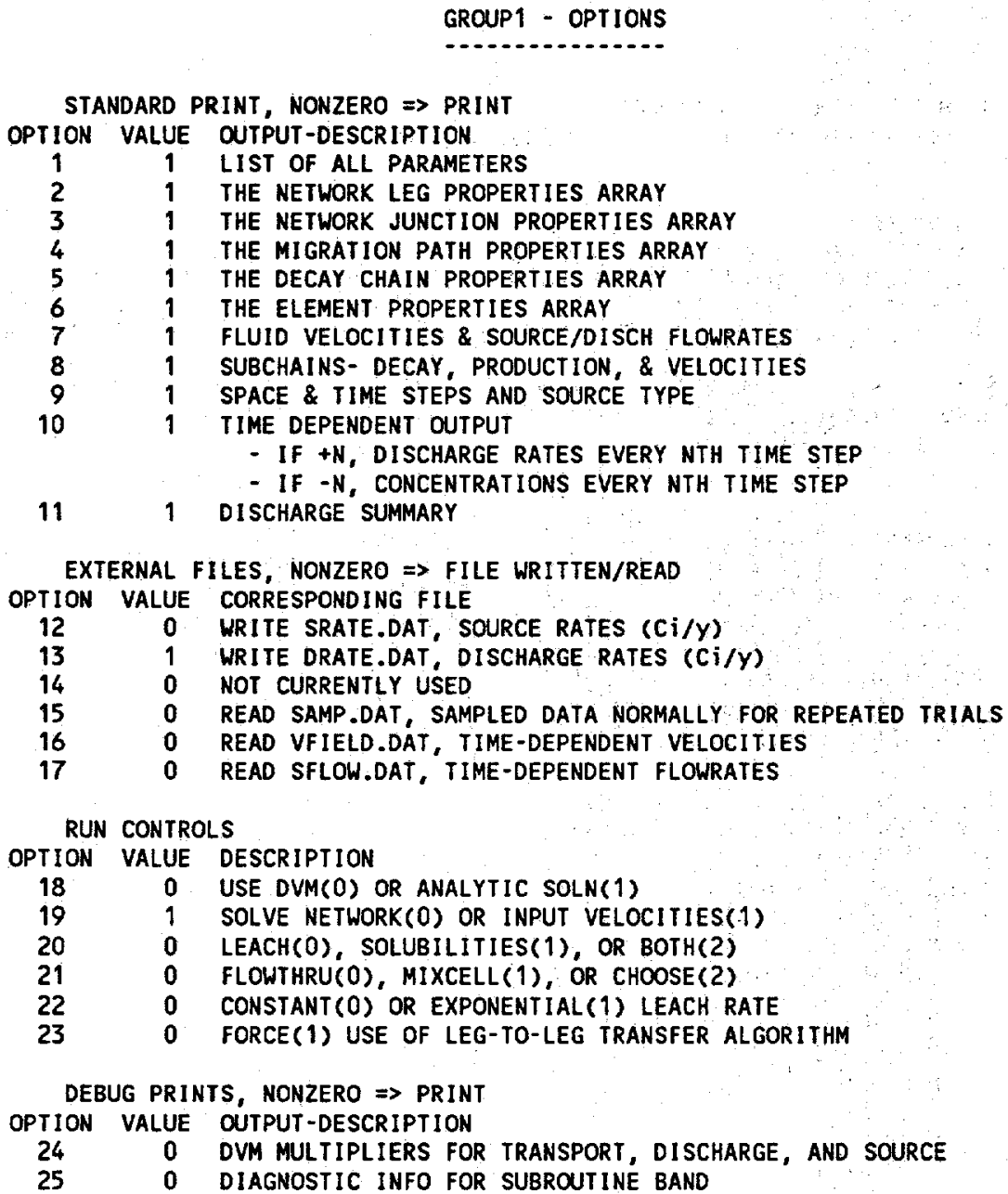

DEBUG PRINTS, NONZERO $\Rightarrow>$ PRINT

OPTION VALUE OUTPUT-DESCRIPTION

2400 DVM MULTIPLIERS FOR TRANSPORT, DISCHARGE, AND SOURCE

250 DIAGNOSTIC INFO FOR SUBROUTINE BAND 


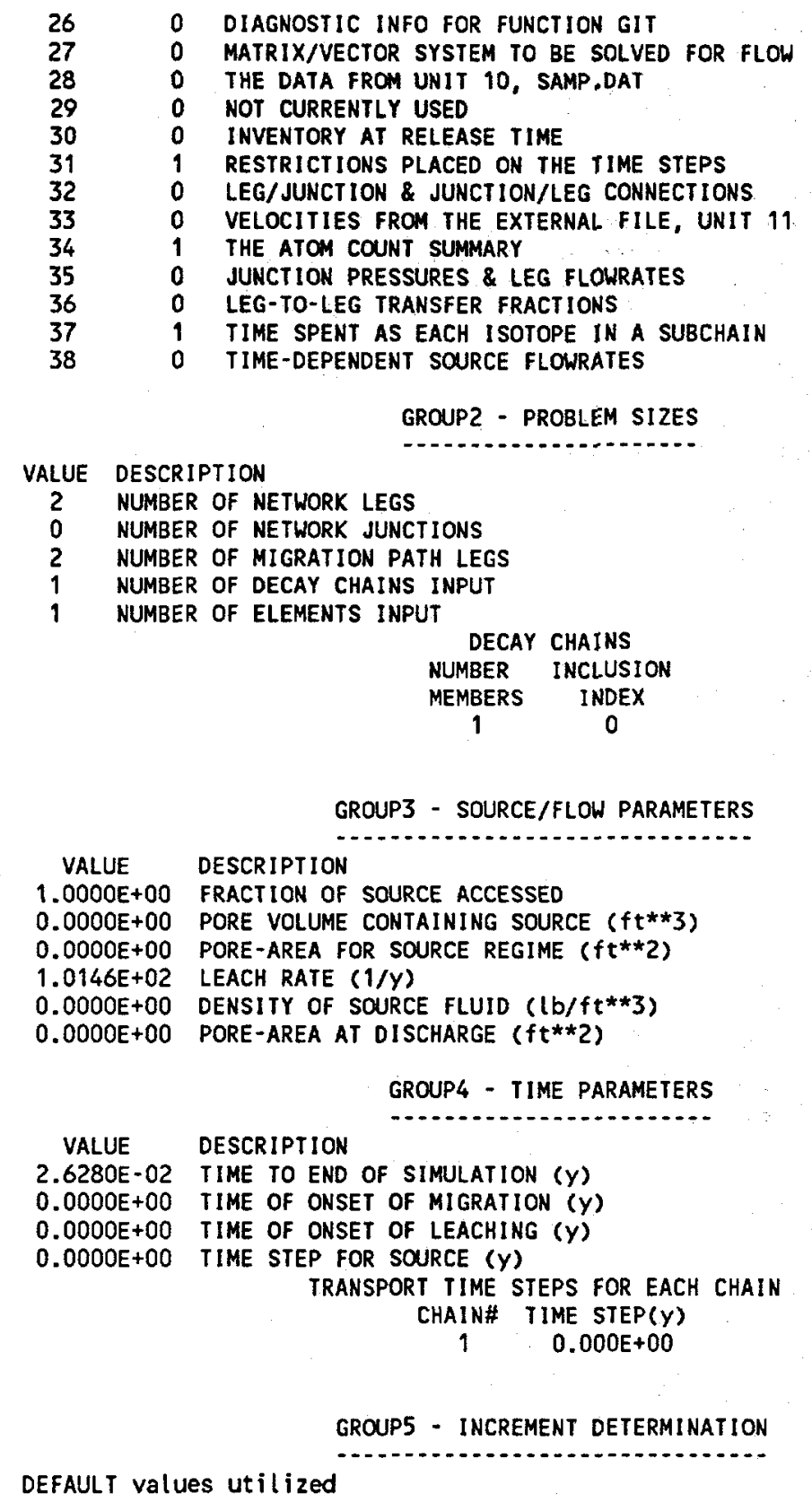

Network Properties Array 


$\begin{array}{ccccccc}\text { Leg } & \begin{array}{c}\text { Inlet } \\ \text { jet }\end{array} & \begin{array}{c}\text { Outlet } \\ \text { jct }\end{array} & \begin{array}{c}\text { Length } \\ (f t)\end{array} & \begin{array}{r}\text { Area } \\ (s q-f t)\end{array} & \begin{array}{c}\text { Hydraulic } \\ K \text { (ft/d) }\end{array} & \begin{array}{c}\text { Brine } \\ \text { Conc. }\end{array} \\ 1 & 1 & 2 & 9.843 E-02 & 0.000 E+00 & 0.000 E+00 & 0.000 E+00 \\ 2 & 2 & 3 & 9.843 E-01 & 0.000 E+00 & 0.000 E+00 & 0.000 E+00\end{array}$

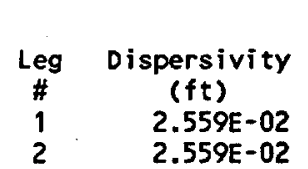

Migration Path Properties Array Space Step Diffusion? Mobile (ft) No/Yes $=0 / 1$ Porosity

$0.000 E+00$

$0.000 E+00$
Immobile

Porosity

$0.000 E+00$

$1.400 \mathrm{E}-01$
Mass Xfer Coef $(1 / y)$

$0.000 \mathrm{E}+00$

$3.013 \mathrm{E}+02$
Velocity

( $\mathrm{ft} / \mathrm{y}$ )

4.609E+02

$4.609 \mathrm{E}+02$

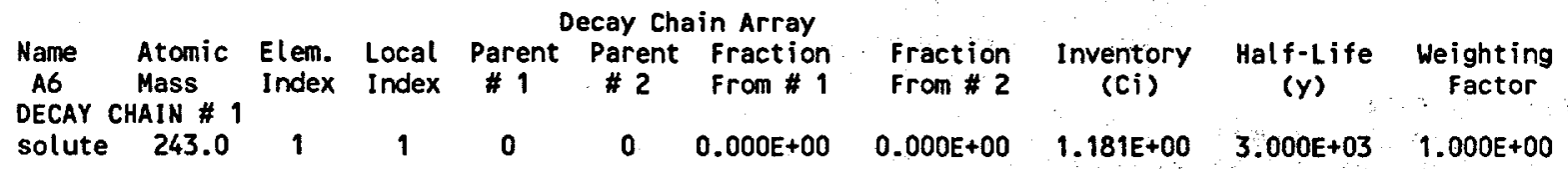

Element Properties Array

$\begin{array}{ccccc}\begin{array}{c}\text { Elem. } \\ \text { Index }\end{array} & \begin{array}{c}\text { Solubility } \\ \text { (g/g) }\end{array} & \begin{array}{c}\text { Leg } \\ \text { \# }\end{array} & \begin{array}{c}\text { Mobil } \\ \text { Rd }\end{array} & \begin{array}{c}\text { Immobile } \\ \text { Rd }\end{array} \\ 1 & 0.000 E+00 & 1 & 1.000 E+00 & 0.000 E+00 \\ & & 2 & 2.000 E+00 & 3.786 E+00\end{array}$

\begin{tabular}{|c|c|}
\hline FOR STEA & ATE FLOW \\
\hline $\begin{array}{l}\text { LEG NO. } \\
\qquad \begin{array}{l}1 \\
2\end{array}\end{array}$ & $\begin{array}{c}\text { PORE VELOCITY } \\
f t / y \\
4.6089 E+02 \\
4.6089 E+02\end{array}$ \\
\hline
\end{tabular}

PATH LENGTH FROM SOURCE BOUNDARY $(f t)=9.8425 E-01$ AVERAGE FLUID VELOCITY $(\mathrm{ft} / \mathrm{Y})=4.6089 \mathrm{E}+02$

FLOW RATE THROUGH SOURCE $=0.0000 E+00 \mathrm{ft} * * 3 / \mathrm{y}$ FLOW RATE AT DISCHARGE $=0.0000 E+00 \mathrm{ft} * * 3 / \mathrm{y}$

THE DETERMINATION OF THE TIME STEP FOR SOURCE PROCEEDS AS FOLLOWS:

LEACH-PULSE RESTRICTION, DT1 $=1.9713 \mathrm{E}-03 \mathrm{y}$

HALF-LIFE RESTRICTION, DT2 $=2.6280 \mathrm{E}-02 \mathrm{y}$

FORCING NOMINAL \# STEPS, DTMAX $=1.3140 E-03 y$

*** FINAL CHOICE, DT $=1.3140 E-03 y$

STEPS IN FINDING TRANSPORT TIME STEP:

MAXIMUM POSSIBLE DT $=3.06246 \mathrm{E}-03 \mathrm{y}$

FOR NOMINAL \# OF STEPS DT $=\mathbf{8 . 7 6 0 0 0 E - 0 4 y}$

DECAY/PRODUCTION FACTORS AND VELOCITIES FOR EACH SUBCHAIN TRANSPORTED SUBCHAIN \#

DESCRIPTION DECAY/PRODUCTION 
VELOCITY INFORMATION FOR EACH SUBCHAIN

LEG 1

\# MIN VELOCITY

$1 \quad 2.20405 E+02$ LEG 2

\# MIN VELOCITY

MEAN VELOCITY

MAX. VELOCITY

MEAN COURANT NO.

$1 \quad 6.03964 \mathrm{E}+01$

MEAN VELOCITY

$2.30445 E+02$

MAX. VELOCITY $2.87140 E+01$

SPACE STEPCLEG

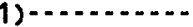

\# OF BLOCKS(LEG 1).........

4.00493E+02

MEAN COURANT NO.

$1.64080 \mathrm{E}+00$

\# OF CATCHER BLOCKSCLEG

1) $-\ldots \ldots$

$D X=1.40607 \mathrm{E}-02 \mathrm{FT}$

$N X=7$

$N C=0$

SPACE STEP(LEG 2) $\ldots \ldots \ldots \ldots \ldots$ DX $=1.23031 \mathrm{E}-01 \mathrm{FT}$

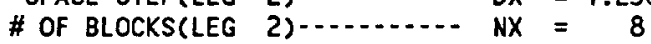

\# OF CATCHER BLOCKS(LEG 2) $\cdots \ldots \ldots$ NC $=0$

TOTAL NUMBER OF GRID BLOCKS- $-N T X=15$

WITH TOTAL CATCHER BLOCKS--.. NEX $=0$

THE TIME STEP.............. DT $=8.76000 E-04 \mathrm{Y}$

FLOWTHRU SOURCE MODEL

1

RADIONUCLIDE DISCHARGE RATE (CI/Y)

YEAR Solute

$0.0000 E+00 \quad 0.0000 E+00$

$8.7600 E-04 \quad 0.0000 E+00$

$1.7520 \mathrm{E}-03 \quad 0.0000 E+00$

2.6280E-03 1.8857E-01

$3.5040 E-03 \quad 2.6177 E+00$

$4.3800 \mathrm{E}-03 \quad 8.6107 \mathrm{E}+00$
$5.2560 \mathrm{E}-03$

$5.2560 \mathrm{E}-03 \quad 1.8124 \mathrm{E}+01$

6. $1320 \mathrm{E}-03 \quad 3.0178 \mathrm{E}+01$

7. $0080 E-03 \quad 4.3460 E+01$

$\begin{array}{ll}7.8840 E-03 & 5.6750 E+01\end{array}$

$8.7600 E-03 \quad 6.9139 E+01$

$9.6360 \mathrm{E}-03 \quad 8.0075 \mathrm{E}+01$

$1.0512 \mathrm{E}-02 \quad 8.9317 \mathrm{E}+01$

$1.1388 E-02 \quad 9.6787 E+01$

$1.2264 \mathrm{E}-02 \quad 1.0207 \mathrm{E}+02$

$1.3140 E-02$ 1.0447E+02

$1.4016 \mathrm{E}-02 \quad 1.0288 \mathrm{E}+02$

$1.4892 \mathrm{E}-02 \quad 9.7108 \mathrm{E}+01$

$1.5768 \mathrm{E}-02 \quad 8.7932 \mathrm{E}+01$

$1.6644 E-02 \quad 7.6640 E+01$

$1.7520 E-02 \quad 6.4556 E+01$

$1.8396 \mathrm{E}-02 \quad 5.2760 \mathrm{E}+01$

$1.9272 E-02 \quad 4.1989 E+01$

$2.0148 \mathrm{E}-02 \quad 3.2642 \mathrm{E}+01$

2.1024E-02 2.4854E+01 
2.1900E-02 1.8579E+01

$2.2776 \mathrm{E}-02 \quad 1.3661 \mathrm{E}+01$

$2.3652 \mathrm{E}-02 \quad 9.8975 \mathrm{E}+00$

$2.4528 E-02 \quad 7.0758 E+00$

2.5404E-02 4.9977E+00

$2.6280 E-02 \quad 3.4914 E+00$

$2.7156 E-02 \quad 2.4147 E+00$

\author{
ATOM COUNT AT 2.7594E-02 YEARS \\ SOURCE IN DISCHARGED \\ $5.965 \mathrm{E}+21 \quad 5.943 \mathrm{E}+21$ \\ $5.943 E+21$
$5.943 E+21$ \\ MOBILE PHASE \\ $5.604 E+18$ \\ IMMOBILE PHASE \\ $1.660 E+19$ \\ solute
TorAL \\ $5.965 E+21$ \\ $5.604 E+18$ \\ $1.660 E+19$ \\ DISCHARGE SUMMARY FOR ALL 1 ISOTOPES
ISOTOPE PEAK-RATE PEAK-TIME \\ PEAK-RATE PEAK-TIME TOTAL(Ci) TOTAL(WEIGHTED) \\ CHAIN 1 \\ solute $\quad 1.0447 E+02 \quad 1.3140 E-02 \quad 1.1731 E+00 \quad 1.1731 E+00$ \\ TOTAL (WE IGHTED) $=1.1731 \mathrm{E}+00$ \\ $* * * *$ NORMAL TERMINATION $* * * *$
}




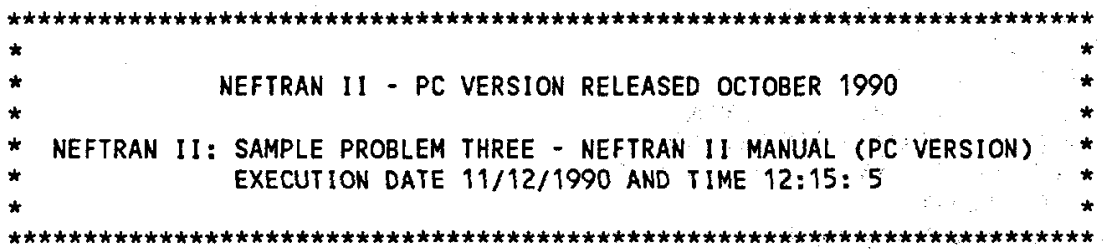

PARAMETERS

GROUP1 - OPTIONS

STANDARD PRINT, NONZERO $\Rightarrow$ PRINT

OPTION VALUE OUTPUT-DESCRIPTION

11 LIST OF ALL PARAMETERS

21 THE NETWORK LEG PROPERTIES ARRAY

31 THE NETWORK JUNCTION PROPERTIES ARRAY

41 THE MIGRATION PATH PROPERTIES ARRAY

51 THE DECAY CHAIN PROPERTIES ARRAY

61 THE ELEMENT PROPERTIES ARRAY

71 FLUID VELOCITIES \& SOURCE/DISCH FLOWRATES

81 SUBCHAINS- DECAY, PRODUCTION, \& VELOCITIES

91 SPACE \& TIME STEPS AND SOURCE TYPE

101 TIME DEPENDENT OUTPUT

- IF + N, DISCHARGE RATES EVERY NTH TIME STEP

11

- IF -N, CONCENTRATIONS EVERY NTH TIME STEP

EXTERNAL FILES, NONZERO $\Rightarrow$ FILE WRITTEN/READ

OPTION VALUE CORRESPONDING FILE

1211 WRITE SRATE.DAT, SOURCE RATES $(\mathrm{C} i / \mathrm{Y})$

131 WRITE DRATE.DAT, DISCHARGE RATES $(\mathrm{C} i / \mathrm{Y})$

14 O NOT CURRENTLY USED

15 O READ SAMP.DAT, SAMPLED DATA NORMALLY FOR REPEATED TRIALS

161 READ VFIELD.DAT, TIME-DEPENDENT VELOCITIES

17 READ SFLOW.DAT, TIME-DEPENDENT FLOWRATES

RUN CONTROLS

OPTION VALUE DESCRIPTION

$18 \quad 0$ USE DVM(O) OR ANALYTIC SOLN(1)

191 SOLVE NETWORK(0) OR INPUT VELOCITIES(1)

200 LEACH(0), SOLUBILITIES(1), OR BOTH(2)

2100 FLOWTHRU(0), MIXCELL(1), OR CHOOSE(2)

220 CONSTANT( 0 ) OR EXPONENTIAL( 1 ) LEACH RATE

230 FORCE(1) USE OF LEG-TO-LEG TRANSFER ALGORITHM

DEBUG PRINTS, NONZERO $\Rightarrow$ PRINT

OPTION VALUE OUTPUT-DESCRIPTION

$24 \quad 0$ DVM MULTIPLIERS FOR TRANSPORT, DISCHARGE, AND SOURCE

250 DIAGNOSTIC INFO FOR SUBROUTINE BAND 


$\begin{array}{lll}26 & 0 & \text { DIAGNOSTIC INFO FOR FUNCTION GIT } \\ 27 & 0 & \text { MATRIX/VECTOR SYSTEM TO BE SOLVED FOR FLOW } \\ 28 & 0 & \text { THE DATA FROM UNIT 10, SAMP.DAT } \\ 29 & 0 & \text { NOT CURRENTLY USED } \\ 30 & 0 & \text { INVENTORY AT RELEASE TIME } \\ 31 & 1 & \text { RESTRICTIONS PLACED ON THE TIME STEPS } \\ 32 & 0 & \text { LEG/JUNCTION \& JUNCTION/LEG CONNECTIONS } \\ 33 & 1 & \text { VELOCITIES FROM THE EXTERNAL FILE, UNIT } 11 \\ 34 & 1 & \text { THE ATON COUNT SUMMARY } \\ 35 & 0 & \text { JUNCTION PRESSURES \& LEG FLOWRATES } \\ 36 & 0 & \text { LEG-TO-LEG TRANSFER FRACTIONS } \\ 37 & 0 & \text { TIME SPENT AS EACH ISOTOPE IN A SUBCHAIN } \\ 38 & 0 & \text { TIME-DEPENDENT SOURCE FLOWRATES }\end{array}$

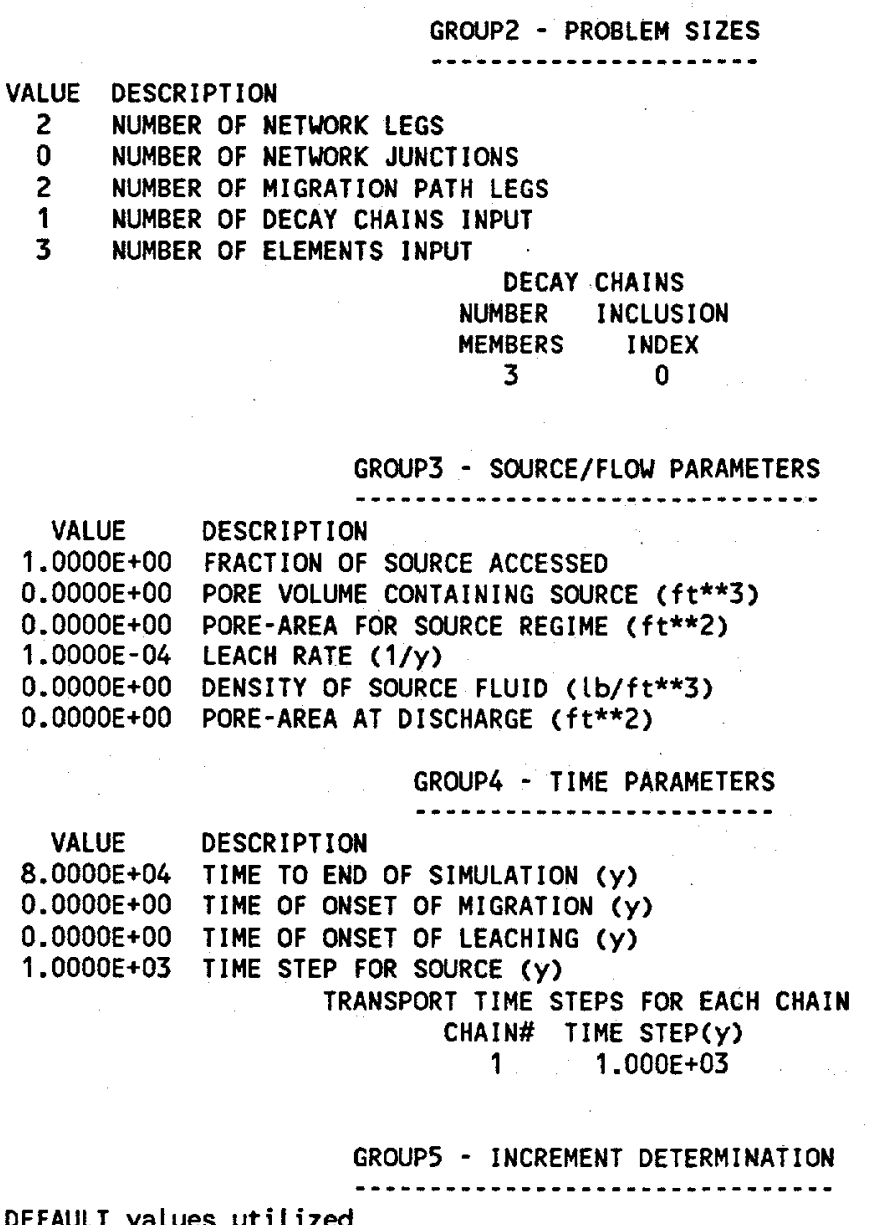

Network Properties Array 


$\begin{array}{ccccrrr}\text { Leg } & \begin{array}{c}\text { Inlet } \\ \text { jct }\end{array} & \begin{array}{c}\text { Outlet } \\ \text { jct }\end{array} & \begin{array}{c}\text { Length } \\ (f t)\end{array} & \begin{array}{r}\text { Area } \\ (\mathrm{sq}-\mathrm{ft})\end{array} & \begin{array}{c}\text { Hydraulic } \\ \mathrm{K}(\mathrm{ft} / \mathrm{d})\end{array} & \begin{array}{c}\text { Brine } \\ \text { Conc. }\end{array} \\ 1 & 1 & 2 & 1.500 E+00 & 0.000 E+00 & 0.000 E+00 & 0.000 E+00 \\ 2 & 2 & 3 & 6.560 E+02 & 0.000 E+00 & 0.000 E+00 & 0.000 E+00\end{array}$

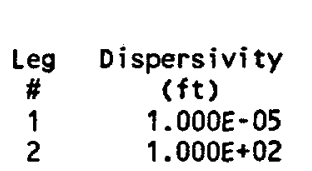

Migration Path Properties Array Space Step Diffusion? Mobile ( $f t$ ) No/Yes $=0 / 1$ Nobile $0.000 E+00$

$1.500 E+00$ o $\quad 0.000 \mathrm{E}+00$

$0 \quad 0.000 E+00$
Immobile Porosity $0.000 E+00$ $0.000 E+00$
Mass Xfer Coef $(1 / y)$

$0.000 E+00$

$0.000 E+00$
Velocity $(\mathrm{ft} / \mathrm{y})$

$0.000 E+00$

$0.000 E+00$

\begin{tabular}{|c|c|c|c|c|c|c|c|c|c|c|}
\hline \multicolumn{11}{|c|}{ Decay Chain Array } \\
\hline $\begin{array}{l}\text { Name } \\
\text { A6 } \\
\text { DECAY }\end{array}$ & $\begin{array}{c}\text { Atomic } \\
\text { Mass } \\
\text { CHAIN \# } 1\end{array}$ & $\begin{array}{l}\text { Elem. } \\
\text { Index }\end{array}$ & $\begin{array}{l}\text { Local } \\
\text { Index }\end{array}$ & $\begin{array}{c}\text { Parent } \\
\# 1\end{array}$ & $\begin{array}{c}\text { Parent } \\
\# 2\end{array}$ & $\begin{array}{l}\text { Fraction } \\
\text { From \# }\end{array}$ & $\begin{array}{l}\text { Fraction } \\
\text { From \#2 }\end{array}$ & $\begin{array}{c}\text { Inventory } \\
\text { (Ci) }\end{array}$ & $\begin{array}{c}\text { Half }-\mathrm{Life} \\
(y)\end{array}$ & $\begin{array}{l}\text { Weight ing } \\
\text { Factor }\end{array}$ \\
\hline $\begin{array}{l}\text { AM243 } \\
\text { OU239 }\end{array}$ & & $\begin{array}{l}1 \\
2\end{array}$ & $\begin{array}{l}1 \\
2\end{array}$ & $\begin{array}{l}0 \\
1\end{array}$ & $\begin{array}{l}0 \\
0\end{array}$ & & & & & $\begin{array}{l}.000 E+00 \\
.000 E+00\end{array}$ \\
\hline & & & & 2 & & & & 12 & & $n n n F+n n$ \\
\hline
\end{tabular}

Element Properties Array

$\begin{array}{ccccc}\begin{array}{c}\text { Elen. } \\ \text { Index }\end{array} & \begin{array}{c}\text { Solubility } \\ (\mathrm{g} / \mathrm{g})\end{array} & \begin{array}{c}\text { Leg } \\ 1\end{array} & \begin{array}{c}\text { Mobil } \\ \text { Rd }\end{array} & \begin{array}{c}\text { Immobile } \\ \text { Rd }\end{array} \\ & 0.000 \mathrm{E}+00 & 1 & 1.900 \mathrm{E}+01 & 0.000 \mathrm{E}+00 \\ & & 2 & 1.900 \mathrm{E}+01 & 0.000 \mathrm{E}+00 \\ 2 & 0.000 \mathrm{E}+00 & 1 & 1.900 \mathrm{E}+01 & 0.000 \mathrm{E}+00 \\ & & 2 & 1.900 \mathrm{E}+01 & 0.000 \mathrm{E}+00 \\ 3 & 0.000 \mathrm{E}+00 & 1 & 1.900 \mathrm{E}+01 & 0.000 \mathrm{E}+00 \\ & & 2 & 1.900 \mathrm{E}+01 & 0.000 \mathrm{E}+00\end{array}$

TIME-DEPENDENT VELOCITIES

$\begin{array}{ccccc}\begin{array}{c}\text { Field } \\ \text { Index }\end{array} & \text { Time }(y) & \begin{array}{c}\text { Leg } \\ \text { Index }\end{array} & \begin{array}{c}\text { Velocity } \\ (\mathrm{ft} / \mathrm{y})\end{array} & \begin{array}{c}\text { Saturation } \\ \text { Fraction }\end{array} \\ 1 & 50000.0 & 1 & 9.80000 \mathrm{E}-02 & 1.000 \mathrm{E}+00 \\ & & 2 & 9.80000 \mathrm{E}-02 & 1.000 \mathrm{E}+00 \\ 2 & 80000.0 & 1 & 1.96000 \mathrm{E}-01 & 1.000 \mathrm{E}+00 \\ & & 2 & 1.96000 \mathrm{E}-01 & 1.000 \mathrm{E}+00\end{array}$

FOR NONSTEADY-STATE FLOW

FOR NONSTEADY-STATE FLOW

EARLIEST NONDISPERSED BREAKTHRU TIME $(y)=6.6939 E+03$ OVER THE TOTAL PATH LENGTH $(f t)=6.5600 E+02$ AVERAGE FLUID VELOCITY ATTAINED $(\mathrm{ft} / \mathrm{t})=9.8000 \mathrm{E}-02$

DECAY/PRODUCTION FACTORS AND VELOCITIES FOR EACH SUBCHAIN TRANSPORTED 
SUBCHAIN \#

1

2

3

4
5

5
6
DESCRIPTION

(DECAY) AM243

(DECAY) PU239

AM243 >PU239

(DECAY) U235

PU239 >U235

AM243 >PU239 >U235
DECAY/PRODUCTION

9.12755E-01

9.71992E-01

8.59987E-02

9.99999E-01

2.80080E-02

$1.24615 E-03$

VELOCITY INFORMATION FOR EACH SUBCHAIN LEG 1

* MIN VELOCITY

$\# \quad$ MIN VELOCITY
$1.5 .14301 E-03$

MEAN VELOCITY

5.14301E-03

5.15789E-03

MAX. VELOCITY

MEAN COURANT NO.

$5.14301 \mathrm{E}-03$

5.15789E-03

5.15789E-03

5. 15789E-03

5.17278E-03

2.40702E+01

5.17278E-03

2.40702E+01

5.14301E-03

$5.17278 E-03$

$2.40702 E+01$

5.17278E-03

2.40702E+01

5.14301E-03

5.15789E-03

5.17278E-03

2.40702E+01

5.14301E-03

LEG 2

5.15789E-03

5.17278E-03

2.40702E+01

MIN VELOCITY

$-4.19118 \mathrm{E}-02$

$-4.19118 \mathrm{E}-02$

$-4.19118 \mathrm{E}-02$

$-4.19118 \mathrm{E}-02$

$-4.19118 \mathrm{E}-02$

MEAN VELOCITY

5. 15789E-03

MAX. VELOCITY

MEAN COURANT NO.

5.15789E-03

5.22276E-02

$3.43860 \mathrm{E}+00$

5.15789E-03

5.22276E-02

$3.43860 \mathrm{E}+00$

5.22276E-02

$3.43860 \mathrm{E}+00$

5.15789E-03

$5.22276 \mathrm{E}-02$

$3.43860 \mathrm{E}+00$

5.15789E-03

$5.22276 E-02$

$3.43860 E+00$

5.15789E-03

$5.22276 E-02$

$3.43860 \mathrm{E}+00$

SPACE STEP(LEG

\# OF BLOCKS LLEG

\# OF CATCHER BLOCKS (LEG
1) $\ldots \ldots$

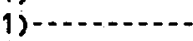
$D X=2.14286 \mathrm{E}-01 \mathrm{FT}$
$N X=6$
1) $\ldots N C=0$

SPACE STEP(LEG 2) $\ldots \ldots \ldots . . . D X=1.50000 E+00 \mathrm{FT}$

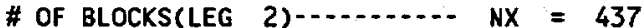

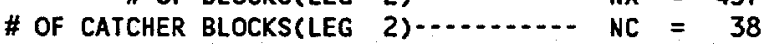

TOTAL NUMBER OF GRID BLOCKS-- NTX $=481$

WITH TOTAL CATCHER BLOCKS -..- NEX $=38$

THE TIME STEP -.......... DT $=1.00000 E+03 \mathrm{Y}$

FLOWTHRU SOURCE MODEL

1

RADIONUCLIDE DISCHARGE RATE (CI/Y)

YEAR AM243 PU239 U235

$\begin{array}{llll}0.0000 E+00 & 0.0000 E+00 & 0.0000 E+00 & 0.0000 E+00\end{array}$

$1.0000 E+03 \quad 0.0000 E+00 \quad 0.0000 E+00 \cdot 0.0000 E+00$

$2.0000 E+03 \quad 0.0000 E+00 \quad 0.0000 E+00 \quad 0.0000 E+00$

$\begin{array}{llll}3.0000 E+03 & 0.0000 E+00 & 0.0000 E+00 & 0.0000 E+00\end{array}$

$\begin{array}{llll}4.0000 E+03 & 0.0000 E+00 & 0.0000 E+00 & 0.0000 E+00\end{array}$

$\begin{array}{llll}5.0000 E+03 & 0.0000 E+00 & 0.0000 E+00 & 0.0000 E+00\end{array}$

$\begin{array}{llll}6.0000 E+03 & 0.0000 E+00 & 0.0000 E+00 & 0.0000 E+00\end{array}$ 


$\begin{array}{llll}7.0000 E+03 & 0.0000 E+00 & 0.0000 E+00 & 0.0000 E+00 \\ 8.0000 E+03 & 0.0000 E+00 & 0.0000 E+00 & 0.0000 E+00 \\ 9.0000 E+03 & 0.0000 E+00 & 0.0000 E+00 & 0.0000 E+00 \\ 1.0000 E+04 & 0.0000 E+00 & 0.0000 E+00 & 0.0000 E+00 \\ 1.1000 E+04 & 0.0000 E+00 & 0.0000 E+00 & 0.0000 E+00 \\ 1.2000 E+04 & 0.0000 E+00 & 0.0000 E+00 & 0.0000 E+00 \\ 1.3000 E+04 & 5.7839 E-10 & 2.9463 E-08 & 2.8939 E-12 \\ 1.4000 E+04 & 2.0287 E-08 & 1.0942 E-06 & 1.1136 E-10 \\ 1.5000 E+04 & 1.9536 E-07 & 1.1192 E-05 & 1.1809 E-09 \\ 1.6000 E+04 & 1.0708 E-06 & 6.5179 E-05 & 7.1286 E-09 \\ 1.7000 E+04 & 4.1851 E-06 & 2.7089 E-04 & 3.0710 E-08 \\ 1.8000 E+04 & 1.2740 E-05 & 8.7704 E-04 & 1.0305 E-07 \\ 1.9000 E+04 & 3.2443 E-05 & 2.3762 E-03 & 2.8931 E-07 \\ 2.0000 E+04 & 7.2056 E-05 & 5.6158 E-03 & 7.0841 E-07 \\ 2.1000 E+04 & 1.4314 E-04 & 1.1872 E-02 & 1.5514 E-06 \\ 2.2000 E+04 & 2.6083 E-04 & 2.3027 E-02 & 3.1164 E-06 \\ 2.3000 E+04 & 4.4068 E-04 & 4.1413 E-02 & 5.8035 E-06 \\ 2.4000 E+04 & 7.0134 E-04 & 7.0161 E-02 & 1.0179 E-05 \\ 2.5000 E+04 & 1.0579 E-03 & 1.1266 E-01 & 1.6920 E-05 \\ 2.6000 E+04 & 1.5265 E-03 & 1.7308 E-01 & 2.6901 E-05 \\ 2.7000 E+04 & 2.1170 E-03 & 2.5557 E-01 & 4.1102 E-05 \\ 2.8000 E+04 & 2.8366 E-03 & 3.6460 E-01 & 6.0665 E-05 \\ 2.0000 E+04 & 3.6864 E-03 & 5.0449 E-01 & 8.6829 E-05 \\ 2.90000 & 1.05 \\ 3.0000+04 & 4.6601 E-03 & 6.7905 E-01 & 1.2087 E-04 \\ 3.1000 E+04 & 5.7483 E-03 & 8.9188 E-01 & 1.6416 E-04 \\ 3.2000 E+04 & 6.9322 E-03 & 1.1452 E+00 & 2.1794 E-04 \\ 3.3000 E+04 & 8.1926 E-03 & 1.4412 E+00 & 2.8352 E-04 \\ 3.4000 E+04 & 9.5029 E-03 & 1.7800 E+00 & 3.6194 E-04 \\ 3.5000 E+04 & 1.0838 E-02 & 2.1617 E+00 & 4.5424 E-04 \\ 3.6000 E+04 & 1.2169 E-02 & 2.5845 E+00 & 5.6117 E-04 \\ 3.7000 E+04 & 1.3469 E-02 & 3.0461 E+00 & 6.8335 E-04 \\ 3.8000 E+04 & 1.4714 E-02 & 3.5434 E+00 & 8.2116 E-04 \\ 3.9000 E+04 & 1.5879 E-02 & 4.0720 E+00 & 9.7473 E-04 \\ 4.0000 E+04 & 1.6947 E-02 & 4.6277 E+00 & 1.1441 E-03 \\ 4.1000 E+04 & 1.7900 E-02 & 5.2050 E+00 & 1.3288 E-03 \\ 4.2000 E+04 & 1.8727 E-02 & 5.7989 E+00 & 1.5286 E-03 \\ 4.3000 E+04 & 1.9420 E-02 & 6.4035 E+00 & 1.7427 E-03 \\ 4.4000 E+04 & 1.9974 E-02 & 7.0134 E+00 & 1.9704 E-03 \\ 4.5000 E+04 & 2.0388 E-02 & 7.6232 E+00 & 2.2108 E-03 \\ 4.6000 E+04 & 2.0664 E-02 & 8.2276 E+00 & 2.4627 E-03 \\ 4.7000 E+04 & 2.0806 E-02 & 8.8216 E+00 & 2.7251 E-03 \\ 4.8000 E+04 & 2.0821 E-02 & 9.4006 E+00 & 2.9966 E-03 \\ 4.9000 E+04 & 2.0717 E-02 & 9.9605 E+00 & 3.2762 E-03 \\ 5.0000 E+04 & 2.0503 E-02 & 1.0497 E+01 & 3.5624 E-03 \\ & & & \end{array}$

OECAY/PRODUCTION FACTORS AND VELOCITIES FOR EACH SUBCHAIN TRANSPORTED SUBCHAIN \# DESCRIPTION DECAY/PRODUCIION

1

2

3

4

$\begin{array}{cc}\text { (DECAY) AM243 } & 9.12755 E-01 \\ & \\ \text { (DECAY) PU239 } & 9.71992 E-01 \\ \text { AM243 >PU239 } & 8.59987 E-02 \\ & \\ \text { (DECAY) U235 } & 9.99999 E-01 \\ \text { PU239 >U235 } & 2.80080 E-02\end{array}$


VELOCITY INFORMATION FOR EACH SUBCHAIN LEG 1

MIN VELOCITY

1.02947E-02

$1.02947 \mathrm{E}-02$

1.02947E-02

$1.02947 E-02$

1.02947E-02

1.02947E-02 LEG 2

MIN VELOCITY

$-5.62509 E-02$

$-5.62509 E-02$

$-5.62509 \mathrm{E}-02$

$-5.62509 E-02$

$-5.62509 \mathrm{E}-02$

MEAN VELOCITY

1.03158E-02

$1.03158 \mathrm{E}-02$

$1.03158 E-02$

$1.03158 \mathrm{E}-02$

$1.03158 \mathrm{E}-02$

1.03158E-02

MAX. VELOCITY

$1.03368 E-02$

MEAN COURANT NO.

$1.03368 E-02$

$1.03368 \mathrm{E}-02$

$1.03368 \mathrm{E}-02$

$1.03368 E-02$

4.81403E+01

4.81403E+01

4.81403E+01

$4.81403 \mathrm{E}+01$

$1.03368 \mathrm{E}-02$

4.81403E+01

MEAN VELOCITY

1.03158E-02

1.03158E-02

$1.03158 \mathrm{E}-02$

MAX. VELOCITY

7.68824E-02

MEAN COURANT NO.

$1.03158 \mathrm{E}-02$

$7.68824 \mathrm{E}-02$

$7.68824 E-02$

$7.68824 E-02$

$1.03158 \mathrm{E}-02$

$7.68824 E-02$

87719E+00

$6.87719 E+00$

$6.87719 E+00$

$6.87719 E+00$

1.03158E-02

$6.87719 E+00$

$6.87719 E+00$

SPACE STEP(LEG

\# OF BLOCKS LLEG

1) $-\ldots \ldots$

$7.68824 E-02$

\# OF CATCHER BLOCKS(LEG

1) $-\ldots+\ldots+\infty$

$D X=2.14286 \mathrm{E}-01 \mathrm{FT}$

$N X=6$

$N C=0$

SPACE STEP(LEG 2) $\ldots \ldots \ldots \ldots-D X=1.50000 E+D D F T$

\# OF BLOCKS(LEG 2) $\ldots \ldots \ldots \ldots . . . . . .4 X$

\# OF CATCHER BLOCKS(LEG 2) $\ldots \ldots \ldots$ NC $=38$

TOTAL NUMBER OF GRID BLOCKS-- NTX $=481$

WITH TOTAL CATCHER BLOCKS---- NEX $=38$

THE TIME STEP-...... DT $1.00000 E+03$ Y

FLOWTHRU SOURCE MOOEL

$\begin{array}{llll}5.1000 E+04 & 3.6875 E-02 & 2.0127 E+01 & 7.0499 E-03 \\ 5.2000 E+04 & 4.0540 E-02 & 2.3542 E+01 & 8.5035 E-03 \\ 5.3000 E+04 & 4.2357 E-02 & 2.6189 E+01 & 9.7563 E-03 \\ 5.4000 E+04 & 4.3280 E-02 & 2.8492 E+01 & 1.0947 E-02 \\ 5.5000 E+04 & 4.3547 E-02 & 3.0526 E+01 & 1.2096 E-02 \\ 5.6000 E+04 & 4.3260 E-02 & 3.2290 E+01 & 1.3194 E-02 \\ 5.7000 E+04 & 4.2512 E-02 & 3.3789 E+01 & 1.4237 E-02 \\ 5.8000 E+04 & 4.1383 E-02 & 3.5024 E+01 & 1.5216 E-02 \\ 5.9000 E+04 & 3.9945 E-02 & 3.6000 E+01 & 1.6125 E-02 \\ 6.0000 E+04 & 3.8269 E-02 & 3.6725 E+01 & 1.6960 E-02 \\ 6.1000 E+04 & 3.6414 E-02 & 3.7213 E+01 & 1.7716 E-02 \\ 6.2000 E+04 & 3.4439 E-02 & 3.7477 E+01 & 1.8392 E-02 \\ 6.3000 E+04 & 3.2391 E-02 & 3.7534 E+01 & 1.8987 E-02 \\ 6.4000 E+04 & 3.0310 E-02 & 3.7402 E+01 & 1.9502 E-02 \\ 6.5000 E+04 & 2.8234 E-02 & 3.7100 E+01 & 1.9938 E-02 \\ 6.6000 E+04 & 2.6189 E-02 & 3.6645 E+01 & 2.0297 E-02 \\ 6.7000 E+04 & 2.4198 E-02 & 3.6056 E+01 & 2.0581 E-02 \\ 6.8000 E+04 & 2.2280 E-02 & 3.5353 E+01 & 2.0795 E-02 \\ 6.9000 E+04 & 2.0447 E-02 & 3.4548 E+01 & 2.0941 E-02 \\ 7.0000 E+04 & 1.8709 E-02 & 3.3663 E+01 & 2.1025 E-02\end{array}$


$7.1000 E+04$ $7.2000 E+04$

$7.3000 \mathrm{E}+04$

$7.4000 \mathrm{E}+04$

$7.5000 E+04$

$7.6000 E+04$

$7.7000 E+04$

$7.8000 E+04$

$7.9000 E+04$

$8.0000 E+04$

$8.1000 E+04$

1.7071E-02 3.2709E+01 2.1050E-02

$1.5536 E-02 \quad 3.1699 E+01 \quad 2.1019 E-02$

$1.4107 E-02 \quad 3.0651 E+01 \quad 2.0939 E-02$

1.2780E-02 2.9570E+01 2.0812E-02

$\begin{array}{lll}1.1555 E-02 & 2.8470 E+01 & 2.0643 E-02\end{array}$

1.0428E-02 2.7361E+01 2.0437E-02

$9.3935 E-03 \quad 2.6246 E+01 \quad 2.0195 E-02$

$8.4487 E-03 \quad 2.5138 E+01 \quad 1.9924 E-02$

7.5869E-03 2.4039E+01 1.9626E-02

6.8034E-03 2.2955E+01 1.9304E-02

6.0929E-03 2.1892E+01 1.8961E-02

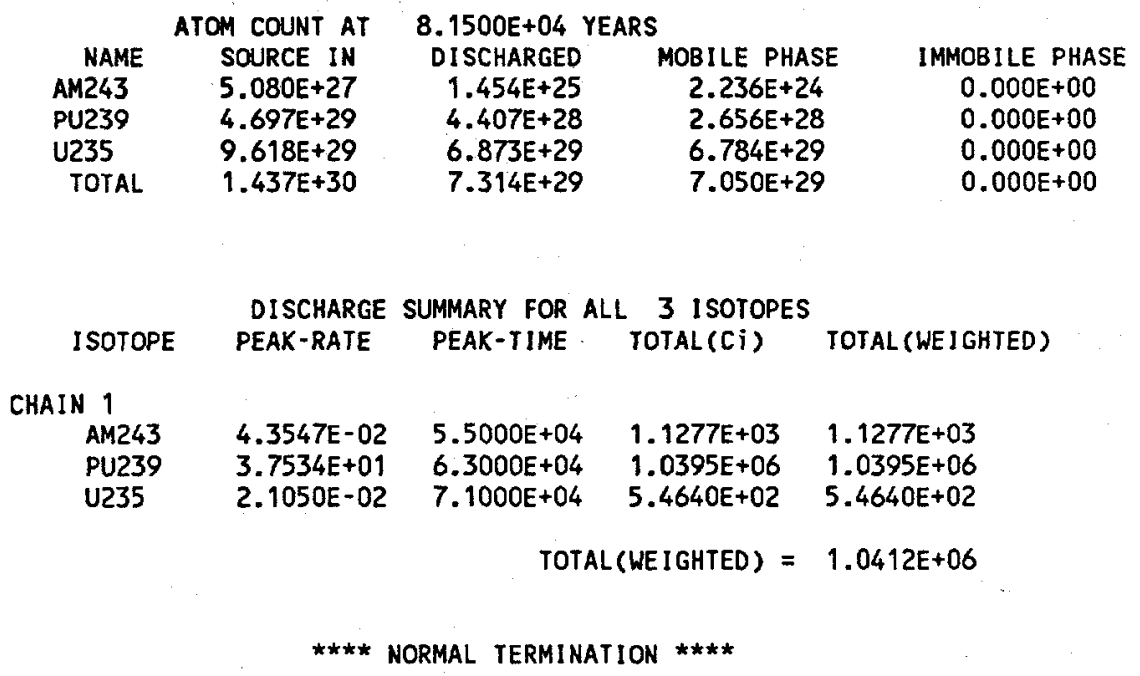




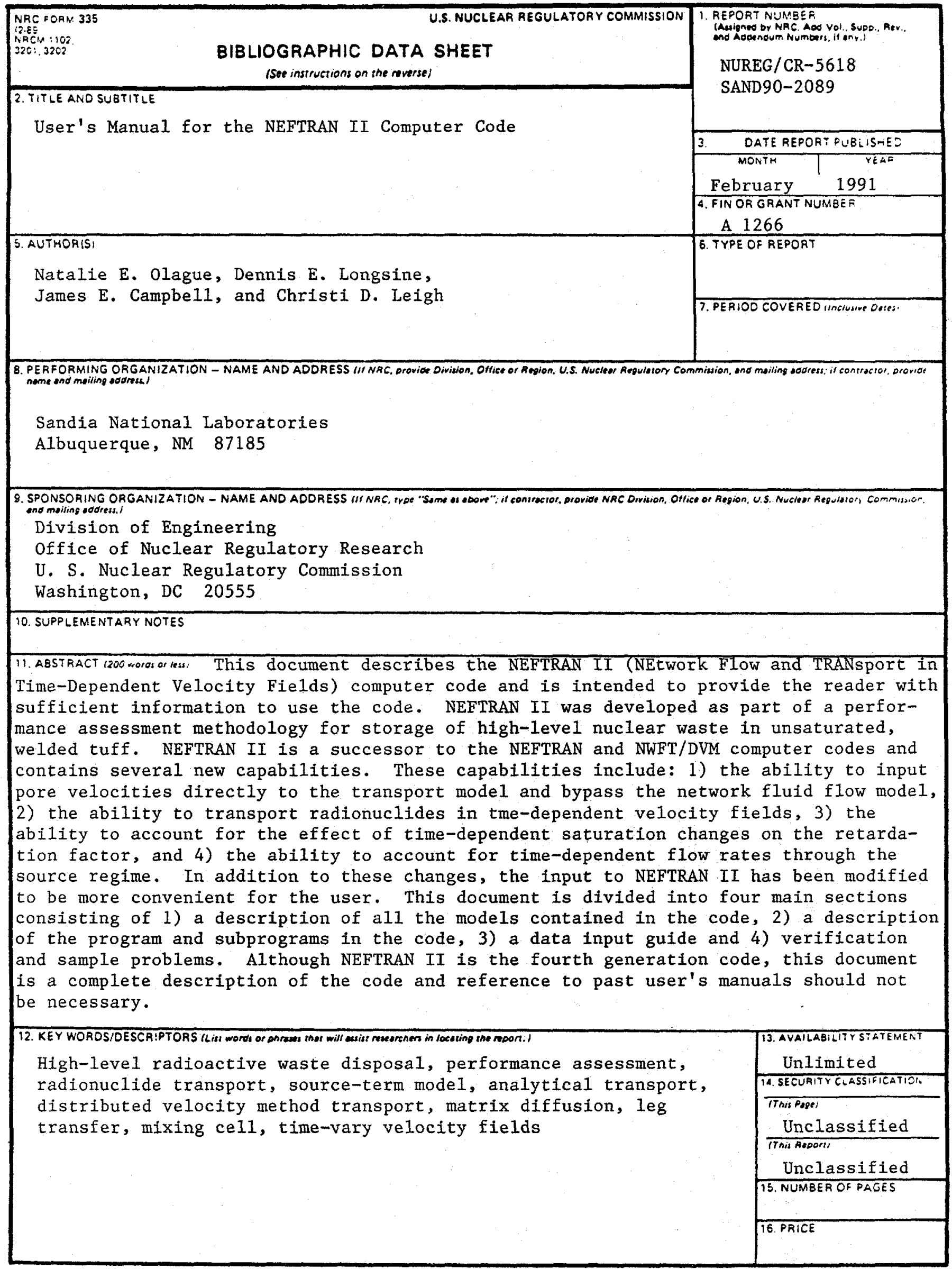

NAC FORN 3351265 ; 